\title{
Speaking of safety
}

The role of communication in managing occupational safety

\author{
Pieter Cornelissen
}




\section{SPEAKING OF SAFETY}

\section{THE ROLE OF COMMUNICATION IN MANAGING OCCUPATIONAL SAFETY}




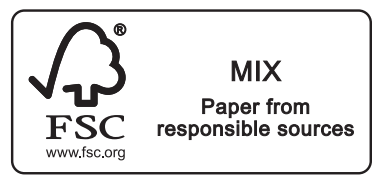

Thesis, University of Twente, 2019

(C) Pieter A. Cornelissen

ISBN: 978-90-365-4706-2

DOI: $10.3990 / 1.9789036547062$

Cover design by Pieter A. Cornelissen

Photo: "Construction helmets" by Peter Heeling (Creative Commons CCO)

Printed by: Gildeprint - The Netherlands 


\title{
SPEAKING OF SAFETY
}

\section{THE ROLE OF COMMUNICATION IN MANAGING OCCUPATIONAL SAFETY}

\author{
PROEFSCHRIFT \\ ter verkrijging van \\ de graad doctor aan de Universiteit Twente, \\ op gezag van de rector magnificus, \\ prof. dr. T.T.M. Palstra, \\ volgens besluit van het College voor Promoties \\ in het openbaar te verdedigen \\ op vrijdag 1 februari 2019 om 16:45.
}

door

Pieter August Cornelissen

geboren op 23 november 1987

te Thorn 
Dit proefschrift is goedgekeurd door de promotor prof. dr. M.D.T. de Jong en de assistent-promotor dr. J.J. van Hoof. 


\section{Samenstelling promotiecommissie}

Voorzitter:

Prof. dr. T.A.J. Toonen

Promotor:

Prof. dr. M.D.T. de Jong

Assistent-promotor:

Dr. J.J. van Hoof

Leden:

Prof. dr. A. Need

Prof. dr. A.T.H. Pruyn

Prof. dr. ir. G.L.L.M.E. Reniers

Prof. dr. ir. J. Dul

Prof. dr. ir. L.A.M. Van Dongen 



\section{Table of contents}

\section{Chapter 1}

General introduction

\section{Chapter 2}

Determinants of safety outcomes and performance: A systematic literature review of research in four high-risk industries

\section{Chapter 3}

Supervisors' beliefs and attitudes towards occupational safety

\section{Chapter 4}

How logical is safety? An institutional logics perspective on occupational safety

\section{Chapter 5}

Enabling employees to work safely: The influence of motivation and ability in the design of safety instructions

\section{Chapter 6}

Towards a broader understanding of safety communication:

Development and initial validation of a measurement instrument

\section{Chapter 7}

General discussion

References

Appendices

Summary

Samenvatting (summary in Dutch)

Bio-bliography 


\section{List of tables}

Table 2.1 Relationships between variables at the cluster level 36

Table 2.2 Relationships between performance and safety outcomes 38

Table 2.3 Relationships between work(place) characteristics \& 40 circumstances, safety outcomes \& performance

Table 2.4 Relationships between climate \& culture, safety outcomes \& performance

Table 2.5 Relationships between management \& colleagues, safety outcomes \& performance

Table 2.6 Relationships between employee demographics, safety outcomes \& performance

Table 2.7 Relationships between external, safety outcomes \& performance

Table 3.1 Overview per managerial level of evaluation points mentioned more than two times

Table 4.1 An overview of key references, the defining characteristics of the seven interinstitutional orders, and their application to 96 safety

Table 4.2 Safety rationale per institutional logic 104

Table 5.1 Scale reliability after factor analysis 126

Table 5.2 Summary of hierarchical regression analysis for variables predicting safety climate

Table 5.3 Summary of hierarchical regression analysis for variables predicting safety performance

Table 6.1 Overview of topics identified through literature review and expert rounds after revision

Table 6.2 Summary of principal component analysis results, indicating extracted factors, corresponding items, factor loadings after rotation for each item, percentage of variance accounted for by each factor, and reliability estimates (Cronbach's $\alpha$ )

Table 6.3 Reliability estimates (Cronbach's $\alpha$ ) for dependent variables

Table 6.4 Overview of means, standard deviations and correlations $\left(r_{\mathrm{s}}\right)$ of communication variables and dependent variables

Table 6.5 Partial correlations $\left(r_{\mathrm{s}}\right)$ when controlling for safety climate, safety motivation and safety knowledge 


\section{List of figures}

Figure 1.1 Types of occupational incidents

Figure 1.2 An integrative model of workplace safety 18

Figure 1.3 Dissertation outline 23

Figure 2.1 Flow diagram of study selection and extracted variables 33

Figure 2.2 Clusters and their associated categories $\quad 35$

Figure 2.3 The most prevalent categories related to performance and safety outcomes and their corresponding number of positive 48 $(+)$, negative $(-)$ and non-significant $(n s)$ relations

Figure 4.1 The institutional orders of the market, profession and corporation and the complexities arising from incompatible 106 prescriptions between them

Figure 5.1 Hypothesised influence of three levels of motivation and ability on safety climate and safety performance

Figure 5.2 Regression model depicting the causal relation of motivation and ability on safety climate and safety performance

Figure 5.3 Regression model depicting the causal relation of the two levels of motivation and ability on safety climate and safety performance 

b) al 1. IN a. 3
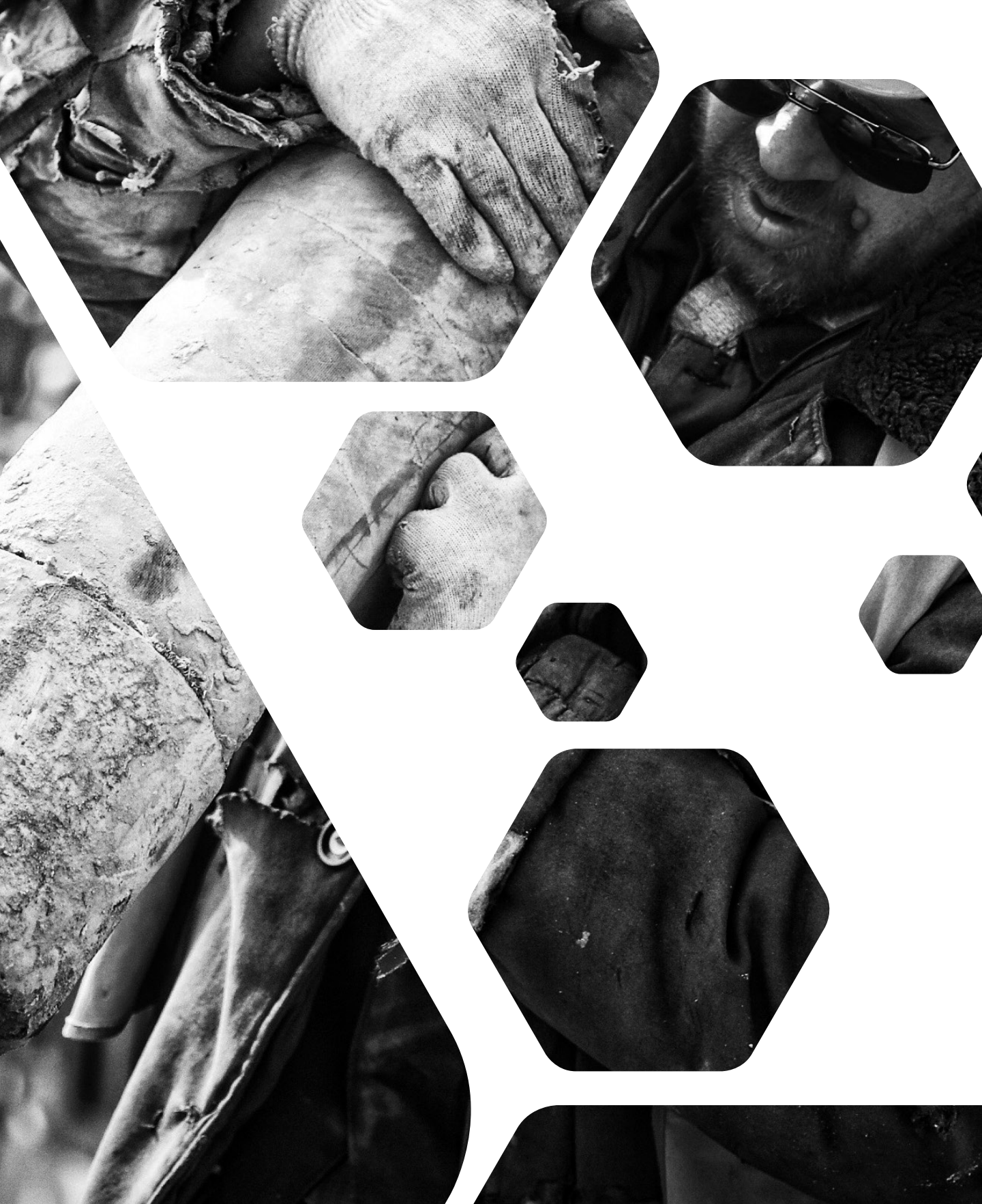


\title{
Chapter 1
}

\section{General introduction}

\author{
'Rules are parts of systems and systems serve humans \\ not humans serve systems. Unfortunately, safety \\ engineers seem to think that humans serve systems.'
}

Rob Long 


\subsection{Preface}

Prypiat, Ukraine, April 26, 1986. North Sea, July 6, 1988. Gulf of Mexico, April 20, 2010. Copiapó, Chili, August 5, 2010. Moerdijk, the Netherlands, January 5, 2011. Tianjin, China, August 12, 2015.

The above are the locations and dates of six occupational incidents: Chernobyl, Piper Alpha, Deepwater Horizon, the Copiapó mining accident, Chemie-Pack, and the Tianjin Port explosions. Six occupational incidents with an enormous impact on organisations and their employees, surrounding communities, and the environment. Six occupational incidents that received extensive media coverage. Six incidents that each sparked the occupational safety debate and are well-known to the general public. However, considering the estimated annual 2.78 million fatalities and 374 million non-fatal occupational injuries and illnesses worldwide due to occupational accidents or work-related diseases, they are just the tip of the iceberg (International Labour Organization [ILO], 2018).

Whereas high-impact accidents in the (offshore) petrochemical, nuclear and mining sectors may be top of mind when one is confronted with these numbers, other sectors like agriculture, construction, logistics, warehouses, and manufacturing must not be ignored. In addition to the impact these kinds of incidents undeniably have on employees, their families and friends, organisations face enormous costs resulting from these incidents and accidents. The ILO estimates that accidents and incidents in the work context cost as much as 3.94 per cent of the yearly global Gross Domestic Product, which would total to an amount between $2.922 .568^{1}$ and $3.178 .942^{2}$ million US dollars in 2017.

The combined physical, emotional, and financial costs of occupational incidents have motivated both academics and practitioners to investigate ways in which the occurrence of these incidents can be prevented and their

\footnotetext{
${ }^{1}$ Estimate based on the financial numbers available through the United Nations http://data.un.org/en/reg/g1.html

${ }^{2}$ Estimate based on the financial numbers available through the World Bank https:// data.worldbank.org/indicator/NY.GDP.MKTP.CD?year_high_desc=true both retrieved on august 6, 2018
} 
impact can be minimised. Advancing insights in the fields of cognition, behaviour, organisations and technics have led to a variety of approaches aimed at improving workplace safety. Yet, despite of over a hundred years of research, more research into occupational safety is much needed. This is not only stressed by the persistence of occupational incidents, but also necessary as work environments become increasingly complex due to technical and technological advancements. Additionally, changes on the labour market such as the diversification of contract types (e.g., Mayhew, Quintan, \& Ferris, 1997; Turner, Stride, Carter, McCaughey, \& Carroll, 2012) and changes due to globalisation (e.g., multilingual teams) may pose challenges for maintaining workplace safety as well. Furthermore, contemporary practitioners still heavily rely on theories and assumptions which have long been criticised or even debunked (cf. Besnard \& Hollnagel, 2014; Manuele, 2011; Marshall, Hirmas, \& Singer, 2018; Swuste, Van Gulijk, \& Zwaard, 2010; Swuste, Van Gulijk, Zwaard, \& Oostendorp, 2014).

This dissertation focuses on how organisations, managers, and employees attempt to influence factors and processes that precede occupational incidents, accidents, and injuries. The studies in this dissertation aim to contribute to increased scientific understanding of occupational safety and ultimately help practitioners create safer work environments. This introduction will continue with a general exploration of the ways in which organisations handle occupational safety (1.2), and safety communication in particular (1.3). It ends with an outline of the different studies in this dissertation (1.4).

\subsection{Safety in the work context}

Researchers and practitioners have tried to minimise the occurrence and impact of occupational incidents and accidents for various reasons and through different approaches. Roughly, these approaches can be divided into approaches that focus on the technical and engineering aspects of work environments and approaches that focus on more 'soft' features of work environments, such as behavioural aspects (e.g., motivation) and organisational influences (e.g., leadership styles).

Organisational interest in safety in the work context first sparked during 
the Industrial Revolution. Around the start of the 20th century the safety movement started to gain real momentum in the Western world, which is thought to be partly due to the high rate of occupational accidents that interfere with labour efficiency, productivity and cost efficiency (Swuste et al., 2010). Around the First World War occupational safety is evolving into a professional field of work and research, and during the first half of the 20th century two hypotheses dominate the then safety debate (Swuste et al., 2010). The first one is the environmental hypothesis which attributes accidents to external factors such as labour conditions and the second one is the individual hypothesis which is based on the premise that some individuals are more prone to accidents than others (Swuste et al., 2010).

After the Second World War the scientific focus concerning occupational safety shifts from a psychological view -which was thought to contribute little to the prevention of accidents- towards 'technology, epidemiology, and ergonomics' (Swuste et al., 2014, p. 24). This shift in views is due to a combination of new scientific insights, accidents resulting from the upscaling of complex chemical production processes, and increased anxiety among the general public concerning industrial disasters and pollution (Swuste et al., 2014; Swuste, Van Gulijk, Zwaard, Lemkowitz, Oostendorp, \& Groeneweg, 2016). From a technological viewpoint, the solution was sought in adapting machines and installations as to prevent that workers' mistakes would lead to accidents. Insights from the medical field, such as the epidemiological triangle, were applied as an answer to the 'conceptual and theoretical weaknesses of psychological research' (Swuste et al., 2014, p. 19). Insights derived from ergonomics research on human interaction with increasingly complex machines during the Second World War were also applied to occupational safety (Swuste et al., 2014). Traditional ergonomics is based on the belief that safety could progress by limiting human contributions through 'proceduralization or automation' (Dekker, 2003). But whereas the focus in safety research after the Second World War shifted towards technical, epidemiological and ergonomic approaches, the professional domain stuck to a human failure approach (Swuste et al., 2014). Swuste and colleagues argue that this is most likely due to the novelty and divergent nature of scientific insights and approaches, combined with an economic perspective. Within the professional domain it was thought that it was easier and cheaper to influence human behaviour than to adapt the work environment and machinery (Swuste et al., 2014). 
Swuste et al. (2016) state that although during the 1970s some steps were made towards integrating the insights and knowledge from different disciplines, the two approaches to safety remained largely distinct. On the one hand safety was addressed through the more technical process safety approach, focusing on systems theory and the technical aspects of production, while on the other hand safety was addressed through a human centred approach (Swuste et al., 2016). The latter approach mirrored the idea of the accident proneness theory to the extent that people, not things, were the cause of accidents. However, the human centred approach did place unsafe acts by employees in perspective by acknowledging the complexity of the organisational context, and social and management and social influences (Swuste et al., 2016).

While in the technical approach to safety incidents are often blamed on unpredictable human behaviour or human errors that degrade a basically safe system (Dekker, 2003), Daniellou, Simard and Boissières (2011) argue that errors are often 'the consequence of the characteristics of the situation' and that human operators detect, manage and correct a great number of errors (p. 11). Dekker (2003) argues that engineered systems should no longer be viewed as inherently safe as no matter how well-designed and well-built a system may be, it inherently has 'a number of hidden vulnerabilities packed into it and its operations' and that resulting accidents should not be explained through human error (p. 212). Instead of being viewed as a cause, human error should be viewed as a symptom of 'failure deeper inside the systems in which people work' (Dekker, 2003, p. 212). Additionally, in more recent years both practitioners and researchers have called for a behavioural and social approach to occupational safety, complementary to the engineering approach, in order to further reduce the number of occupational incidents, accidents, and injuries (e.g., Daniellou et al., 2011; O’Dea \& Flin, 2001; Törner \& Pousette, 2009; Wachter \& Yorio, 2014). Given that incidents, accidents and injuries are still commonplace in contemporary organisations despite technical and engineering advancements, and acknowledging the call by researchers for a complementary approach to safety that focusses on behavioural and social elements, this dissertation will focus on cognitive, behavioural, social, and organisational aspects of safety in the workplace. 


\subsubsection{Indicators of occupational safety}

Traditionally, occupational safety is measured in terms of unsafety: the occurrences of incidents in the work context that might result in injuries and diseases (Daniellou et al., 2011; Hinze, Thurman, \& Wehle, 2013). The rationale behind this is that safety successes are not directly visible or easily measured in terms of visible returns or profits (Mengolini \& Debarberis, 2008). As the terms associated with incidents are used inconsistently in scientific research and sometimes overlap (Khanzode, Maitei, \& Ray, 2012), an overview and description of the most important outcomes and their differences is provided (see Figure 1.1).

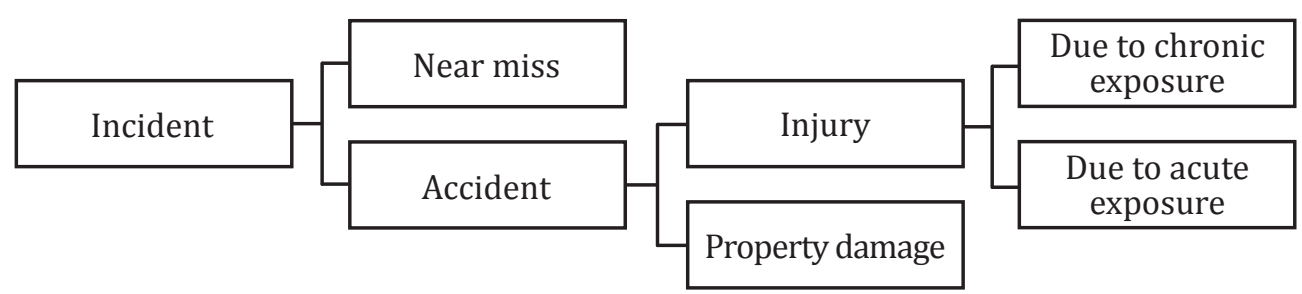

Figure 1.1. Types of occupational incidents

An incident is defined as 'something that happens, especially something unusual or unpleasant' (Oxford Advanced Learner's Dictionary, n.d.). Translated to the occupational context, incidents are those events that are unplanned, undesired, and have a negative effect on the completion of a work-task (OSHA, n.d.). Depending on the outcome of an incident it can be classified as either a near miss or an accident. Near misses are incidents that do not result in either property damage or personal injury, despite having the potential to do so 'given a slight shift in time or position' (OSHA, n.d.). Accidents are incidents that result in injury and/or property damage COSHA, n.d.). An injury to a human being is a bodily wound or trauma resulting from acute or chronic exposure to an energy transfer that exceeds the physiological threshold (Khanzode et al., 2012).

Incidents, near misses, accidents, injuries and property damage are lagging indicators of safety (Pawłowska, 2015). Lagging indicators are relatively easy to measure as they (often) emerge through inspections or employee reports. Although these indicators are well-suited to provide information about the absence of safety in organisations, they do not provide information on the reasons that lead to these outcomes (Hinze et al., 2013). Additionally, as 
incidents occur unexpectedly and at irregular intervals, such lagging indicators are not suited for providing a reliable, stable and reproducible indication of occupational safety within an organisation. It is therefore no wonder that the usefulness of lagging indicators for prevention of future incidents and creating safer workplaces is questioned by a growing number of safety professionals and researchers (e.g., Grabowski, Ayyalasomayajula, Merrick, \& Mccafferty, 2007; Mengolini \& Debarberis, 2008). Instead, it is argued that it would be better to use leading indicators that focus on the input that may eventually lead to negative safety outcomes. While such indicators are harder to measure, they are easier to influence. One such widely used leading indicator for occupational safety in research is safety performance.

\subsubsection{A human, social, and organisational approach to occupational safety}

Within the occupational setting, one can distinguish between three levels on which safety can be analysed: the individual level, the group level, and the organisational level (Guldenmund, 2007; Hofmann, Jacobs, \& Landy, 1995). On the individual level, occupational safety research is concerned with a wide variety of factors, such as personal characteristics, cognitive, attitudinal, and perceptual processes that influence individual behaviour (e.g., Guldenmund, 2007; Hofmann et al., 1995; Siu, Philips, \& Leung, 2003). Building on the previous scientific work Christian, Bradley, Wallace and Burke (2009) developed a model that depicts individual and situational antecedents of safety performance behaviours and safety outcomes (see Figure 1.2). Within their model, safety outcomes can occur in the form of negative events such as accidents and injuries. These negative events are preceded by safety performance. While this term has been used to refer to safety outcomes (e.g., lost time incident frequency or LTIF), it is more commonly used to 'refer to a metric of safety-related behaviors of individuals' (Christian et al., 2009, p. 1104). According to Christian et al. (2009) safety performance is construed out of two factors: safety compliance and safety participation. These two factors are preceded by two person-related factors: safety motivation and safety knowledge. In turn, safety motivation and safety knowledge are preceded by distal factors related to the individual and related to the situation. The situation-related antecedents of safety performance include various safety climate factors and leadership, whereas the distal person-related antecedents include various personality characteristics and job attitudes (Christian et al., 2009). 


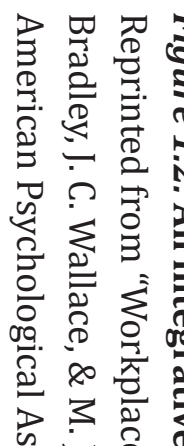

论

․ㅠ.

产. 产这

N

잉

官 常 출

2 क

容怘

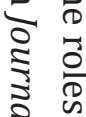

웅

范

จ

जै

ऽั

है

它

जิ ?

क

号

이 3

2 u

을

这.

尝

요요

可
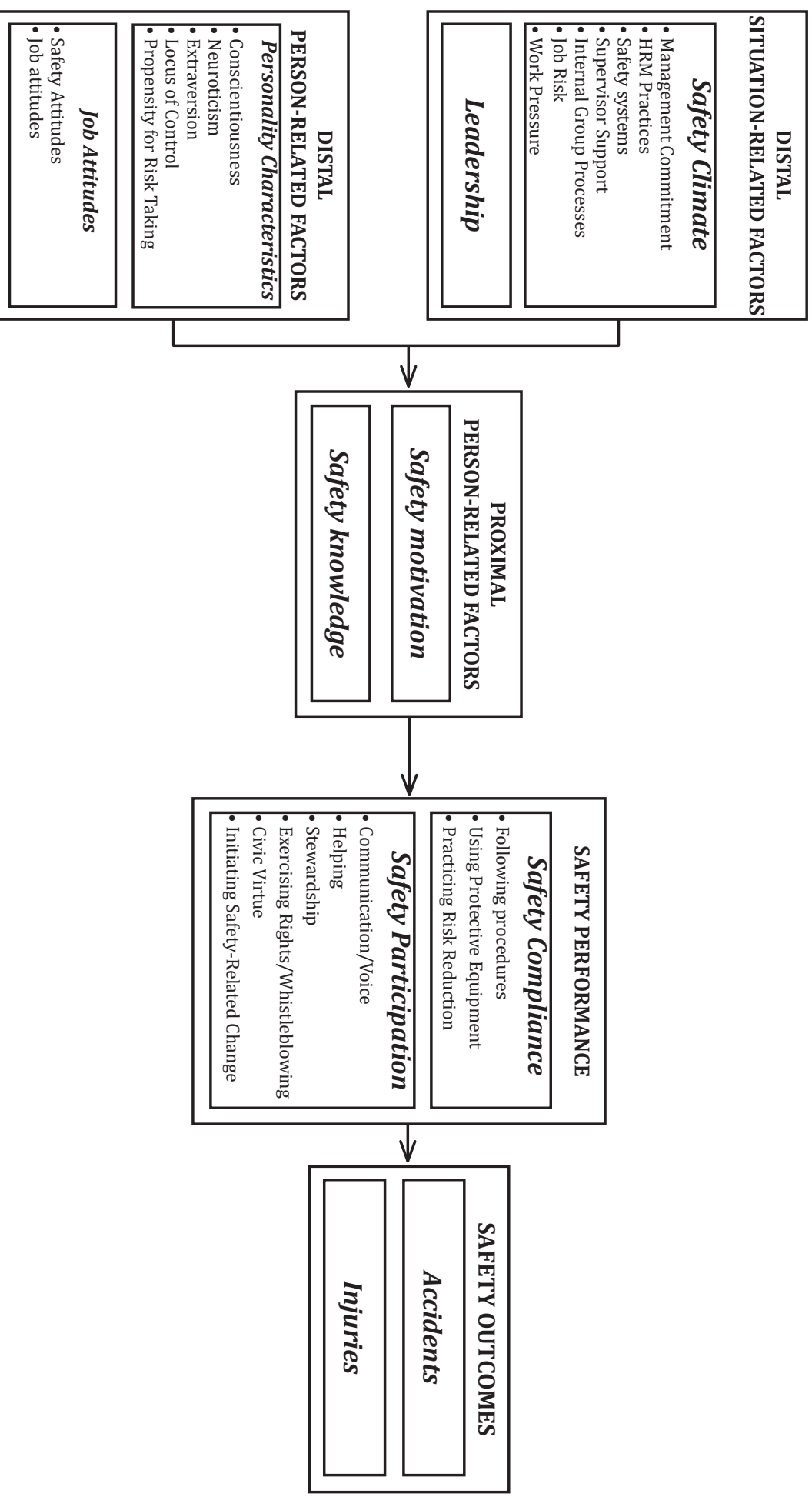
Although individual characteristics are important in understanding and improving occupational safety, the model by Christian et al. (2009) also indicates that it is difficult and undesirable to study these factors without taking group and organisational level factors into account. A regular work situation will present a variety of factors that may affect the individual, such as the motivation to perform a certain behaviour (Andriessen, 1978; Daniellou et al., 2011). The importance of others for an individual's behaviour and intention is also present in the theory of reasoned action (cf. Fishbein \& Ajzen, 1975) and the theory of planned behaviour (cf. Ajzen, 1985) and, among others, demonstrated in the experiments conducted by Milgram (cf. Milgram, 1963). When discussing the influence of 'others' a distinction has to be made between the group level and the organisational level (Guldenmund, 2007; Hofmann et al., 1995). The group level refers to '(behavioural) processes within groups or teams the respondent works in and belongs to, including the team leader or supervisors', whereas the organisational level refers to '(behavioural) processes taking place at higher organisational levels' such as plant management, and senior and top management (Guldenmund, 2007, p. 727). All of these levels have been found to be important for safety performance behaviours (e.g., Andriessen, 1978; Barling, Loughlin, \& Kelloway, 2002; Daniellou et al., 2011; Tucker, Chmiel, Turner, Hershcovis, \& Stride, 2008; Zohar \& Luria, 2003).

The different approaches to safety with their wide variety of studied factors, call for a thorough overview of what has been studied to date. While other researchers have provided such overviews in the form of meta-analyses and systematic literature reviews, they have done so for one specific sector (e.g., Mearns \& Yule, 2009), specific topics (e.g., Clarke, 2013), or applied a historical perspective (e.g., Swuste et al., 2010, 2014, 2016). Therefore this dissertation started with the question:

\section{RQ 1: What is the current status of occupational safety research with regard to determinants of safety outcomes and performance?}

This question served as a starting point and the answer provided directions for subsequent research. 


\subsection{Safety communication}

Communication is essential for the effective organisation and coordination of work between individuals and within groups, especially large and complex groups such as organisations (Quinn \& Dutton, 2005). The importance of communication also stretches to the field of occupational safety, where it has been a long recognised element for the safe execution of work (e.g., Bentley \& Haslam, 2001; Hofmann et al., 1995; Hofmann \& Morgeson, 1999; Mearns, Whitaker, \& Flin, 2003; Vassie \& Lucas, 2001). The term safety communication is mainly associated with the dissemination of information regarding safetyrelated topics between supervisors and subordinates (e.g., DeJoy, Schaffer, Wilson, Vandenberg, \& Butts, 2004; Hofmann \& Morgenson, 1999; Michael, Guo, Wiedenbeck, \& Ray, 2006). Safety communication is viewed by some as a stand-alone variable that might directly affect safety performance (e.g., Alsamadani, Hallowell, \& Javernick-Will, 2013a), while others view safety communication as part of safety climate (e.g., Cheyne, Cox, Oliver, \& Tomás, 1998; Neal, Griffin, \& Hart, 2000), or part of safety culture (e.g., Behm \& Schneller, 2013; Boughaba, Hassane, \& Roukia, 2014). Despite the synonymous use of the terms safety climate and safety culture, Yule (2003) argues that they must be viewed as complementary, yet independent concepts that are reflective of employee beliefs and perceptions at different levels of abstraction.

The focus on the interaction between supervisor(s) and subordinate(s) in safety communication might be explained from a historical perspective. In line with the dominance of the individual hypothesis and the popularity of Taylor's scientific management during the first half of the 20th century, organisations initially focused their communication efforts on instructing employees on the operation of machinery and communicating rules and procedures through posters, safety boards and bulletins (Swuste et al., 2010). Notwithstanding that such a focus on supervisor-subordinate interaction might (still) seem logical from a traditional business and management view on organisations and work, it seems rather limited from other perspectives. Furthermore, research in other areas such as healthcare has shown that it takes more than announcing procedures and rules for them to become effective (e.g, Van Gemert, 2003). And from a communication perspective, the focus on supervisor-subordinate interaction disregards the exchange of information between employees (on the same level) and neglects many other aspects and factors that are thought 
to be important for effective communication, including aspects of the message, media, noise, and unintentional or implicit communication (cf. Axley, 1984; Daft \& Lengel, 1986; Entman, 1993; Nilsen, 1957; Shannon \& Weaver, 1949).

Limited views on communication in occupational safety research and the lack of a clear understanding of how safety relates to other variables thought to be important for occupational safety create the need for a broader and improved understanding of the safety communication. This need is further increased by several ongoing societal and organisational changes. More and more, organisations face the challenges associated with globalisation that might affect occupational safety, such as language barriers and cultural differences (e.g., Alsamadani, Hallowell, \& Javernick-Will, 2013a; Corvalan, Driscoll, \& Harrison, 1994; Sargeant \& Tucker, 2009). Furthermore, organisations are confronted with a changing labour market where self-employment, temporary and part-time contracts have become increasingly commonplace, leading to a decline of people with full-time jobs (Quinlan, 1999). While organisations benefit from fewer permanent employees in terms of flexibility and lower costs (Von Hippel, Mangum, Greenberger, Heneman, \& Skoglind, 1997), these changes can have an adverse effect on occupational safety, due to less familiarity with the work(place) and the organisation, job insecurity, and poorer quality labour relations (e.g., De Cuyper \& De Witte, 2007; Rousseau \& Libuser, 1997; Quinlan, 1999). The challenges of assimilating new employees, maintaining quality standards and a safe work environment faced by organisations, create the need for more safety communication.

Given the (increasing) importance of communication for safety in the workplace, existing ambiguity regarding the concept, and the contemporary narrow view on safety communication in research, this dissertation aims to further explore the concept of safety communication and its underlying aspects. Therefore the following overall research question is formulated:

\section{RQ 2: What is the role of communication in occupational safety?}




\subsection{Outline}

Given that (fatal) accidents are still commonplace in organisations despite advancements in both the technical and engineering domain, and acknowledging the call by researchers for a complementary behavioural and social approach to safety, this dissertation will focus on cognitive, behavioural, social, and organisational aspects of safety in the workplace. The chapters in this dissertation are based on scientific papers. These scientific papers are modified to match the layout, numbering, and spelling of this dissertation but are otherwise included without modifications. A visual representation of the dissertation's outline is presented below (Figure 1.3).

\section{Systematic literature review}

In chapter $\mathbf{2}$ a systematic review of occupational safety literature is presented, making a contribution that is threefold. First, the review identifies and groups factors that have been studied in occupational safety research. Second, the review provides an indication of the scientific strength of the relationships between the identified variables and safety outcomes and safety performance. And third, it assesses the relationships and subsequently categorises them into groups of relationships about which there is consensus, relationships about which there is debate, and existing gaps. The results indicate that despite the amount of research, several factors are still subject to debate or understudied. One of the most interesting clusters emerging from this study is that of the influence of management and colleagues and more specifically, the results regarding the management of safety. In light of organisational efforts to improve occupational safety, these results promised an interesting research avenue which were further explored in chapter 3.

\section{Empirical studies}

Chapter 3 describes a qualitative study which zooms in on two categories from the management and colleagues cluster brought forward by the systematic literature review in chapter 2: management attitudes \& behaviours and management of safety. By means of semi-structured interviews supervisors' perceptions, beliefs, and attitudes regarding the role and 


\section{Chapter 1}

Introduction

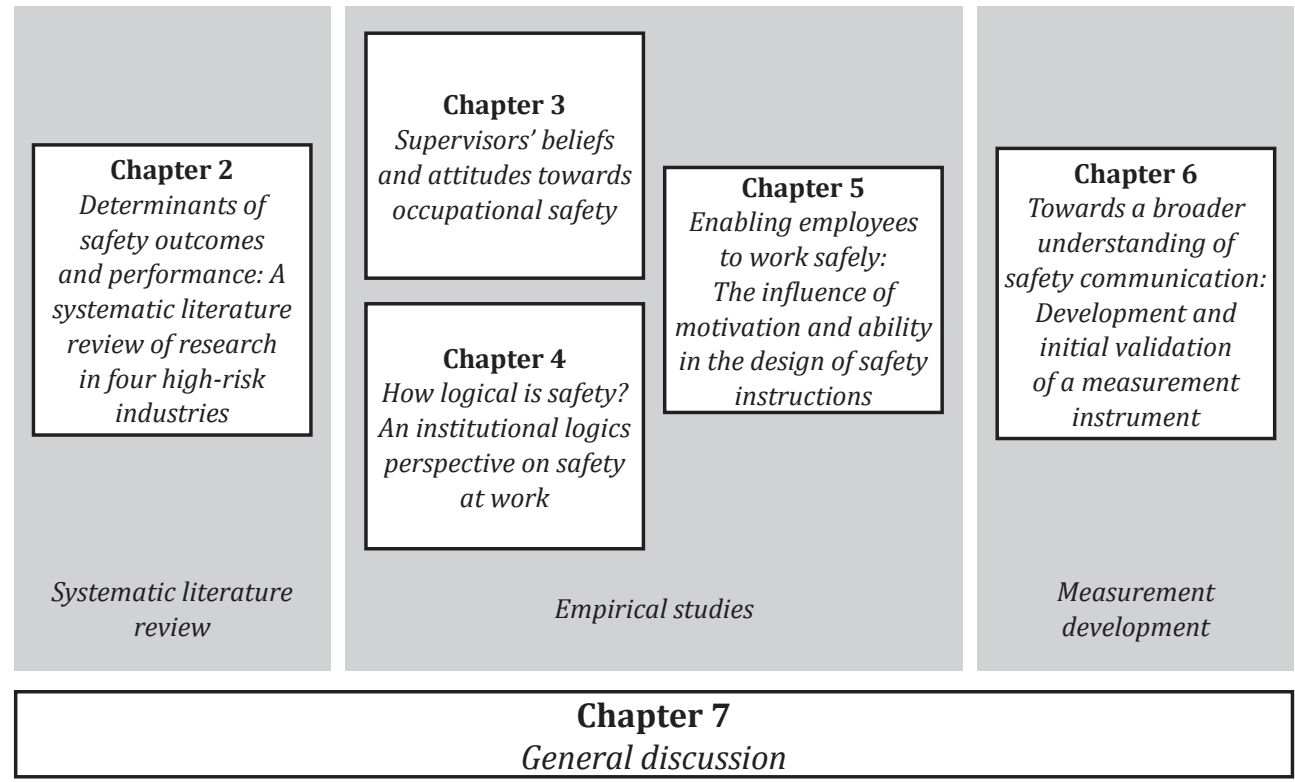

Figure 1.3. Dissertation outline

management of safety in their work are assessed. It becomes apparent that supervisors must balance safety with other objectives, despite the importance of occupational safety in their work. Additionally, differences in safety beliefs and attitudes between managerial levels and individual supervisors are illustrated, as well as these beliefs and attitudes affect safety management. From the various safety management instruments, communication emerges as a crucial factor. Not only do supervisors view communication as an effective mean on its own, they also stress its importance for other safety management instruments.

Chapter 4 presents a theoretical approach to the different rationales that people can hold towards occupational safety. In his critique on the old ergonomics view on safety, Dekker (2003) states that systems provide people with multiple goals that they must pursue simultaneously. People's behaviours and decisions at a given moment are based on their then knowledge, perspective and understanding of the situation, also called 'local rationality' 
(Dekker, 2003). In order to investigate the presence of such different rationales, an institutional logics perspective was applied to qualitative data collected among supervisors. The results illustrate the presence of the different logics of the market, profession, and corporation in the occupational safety context. Furthermore, contradictory viewpoints -so-called institutional complexity- between these three logics and subsequent management approaches emerge.

Chapter 5 bridges the more intrinsic and attitudinal studies described in chapters 3 and 4 and focuses on what elements one should use in order to enable employees to work safely. This study is centred around pinpointing which sublevels of employee motivation and ability safety efforts should be focused. The results indicate that distinguishing between different levels of motivation and ability increased the predictive power of these constructs for safety climate and safety performance, compared to the use of the overall constructs of motivation and ability. From the different levels, only the external level of ability (e.g. creating the right work environment by making the right tools and machinery available) was found to be predictive of safety climate, while for safety performance only the personal level of motivation (e.g. the intrinsic desire or choice to work safely) and the external level of ability held predictive value. The results confront researchers and practitioners with the challenges of how to communicate about occupational safety with employees and how to measure if these efforts are effective.

Chapter $\mathbf{6}$ describes the development of a measurement instrument on safety communication. The importance of occupational safety was not only brought to light by the systematic review in chapter 2 , but also stressed by supervisors in chapter 3 and more implicitly illustrated in chapters 4 and 5. The goal of this study was to develop and provide an initial validation of a quantitative measurement instrument that allows researchers to expand current knowledge about safety communication and its relationship with other safety-related concepts, and practitioners with a practical measure to assess the perceived quality of safety communication and identify (potential) issues related to safety communication within organisations. 
General discussion

In Chapter $\mathbf{7}$ the key findings of the various studies are summarised and theoretical and practical contributions are discussed. Lastly, this chapter reflects upon the limitations of the dissertation and propose directions for future research. 


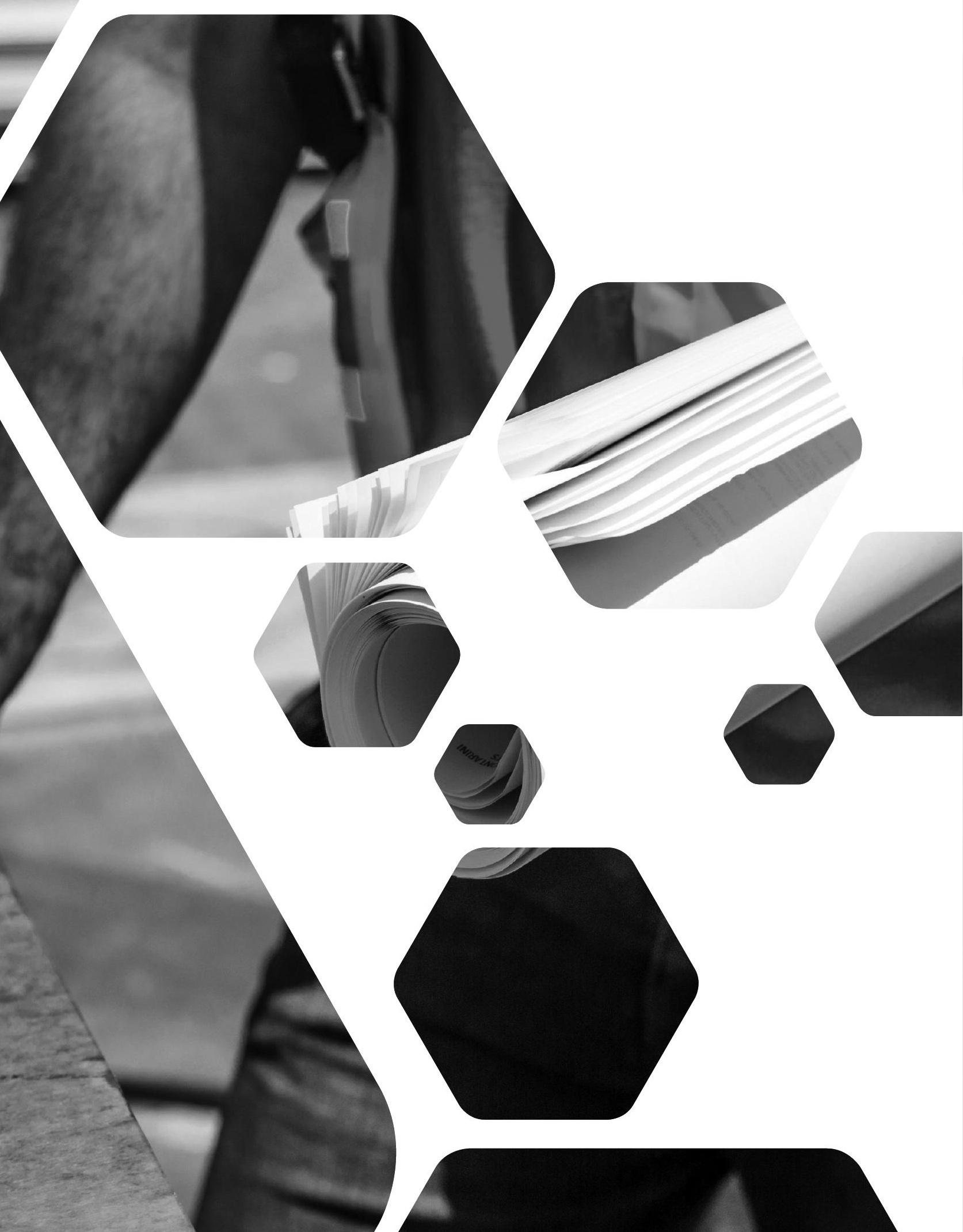




\title{
Chapter 2
}

\section{Determinants of safety outcomes and performance: A systematic literature review of research in four high-risk industries}

\begin{abstract}
'While the individual man is an insoluble puzzle, in the aggregate he becomes a mathematical certainty. You can, for example, never foretell what any one man will do, but you can say with precision what an average number will be up to. Individuals vary, but percentages remain constant. So says the statistician.'
\end{abstract}

Arthur Conan Doyle

Cornelissen, P.A., Van Hoof, J.J., \& De Jong, M.D.T. (2017). Determinants of safety outcomes and performance: A systematic literature review of research in four high-risk industries. Journal of Safety Research, 62, 127 - 141. doi: 10.1016/j.jsr.2017.06.009 


\subsection{Introduction}

The number of occupational accidents exceeds 313 million annually worldwide (International Labour Organization [ILO], 2015), underscoring the relevance of occupational health and safety for organisations. According to ILO (1998) occupational accidents include work-related events that are unexpected or unplanned and result in one or more workers suffering a personal injury, disease or death. These regrettable events have serious physical and emotional consequences for the employees involved, have severe impacts on co-workers, first responders, and families, and result in costs estimated at $4 \%$ of the global gross domestic product (ILO, 2015). The origin of occupational safety as a topic of interest for organisations can be traced back to the $19^{\text {th }}$ century, when rapid industrialisation was characterised by economic, technical, and social changes on an unprecedented scale (Swuste et al., 2010). However, improving safety proved much more complicated than expected, causing a division between the scientific and the corporate worlds (Swuste et al., 2014). Whereas science tried to understand accidents as processes of causes and effects, organisations adhered to their trusted theory of accident proneness: the idea that some people are predisposed to be more susceptible to accidents (Arbous \& Kerrich, 1951). In this study we aim to provide an overview of the most prevalent safety factors studied over the past 35 years, provide an overview of the determinants of safety outcomes, and ultimately bridge the gap between the scientific and the corporate worlds. Whereas previous research provided overviews of the literature from a historical perspective (e.g., Swuste et al., 2010; Swuste et al., 2014), or focused on a specific topic (e.g., Clarke, 2013; Wagstaff \& Lie, 2011), or a specific domain (e.g., Abdul-Aziz \& Hussin, 2003; Mearns \& Yule, 2009), our study delivers a comprehensive overview of the occupational safety literature over the last 35 years, covering a broad range of topics in four different domains (construction, (offshore) petro chemistry, warehouses, and manufacturing). Before we describe our methodology and results, we will provide a short overview of the main variables in occupational safety research literature. Finally, we will critically review our findings and discuss implications for both practice and research, as well as directions for future research. 


\subsubsection{Safety outcomes and performance}

The ultimate end goal in occupational safety is the reduction or -preferablyelimination of negative safety outcomes. These negative safety outcomes come in different forms like near misses, accidents, and injuries. These events are often distinguished from each other based on Heinrich's pyramid (for more information see Heinrich, 1931), which classifies unwanted safety-related events based on their outcomes. We will use a similar, although compressed, classification. We classify negative outcomes that have the potential to inflict harm as incidents, such as near misses and employee errors. We classify incidents that result in property or financial damage as accidents, and we classify accidents that result in mental or physical damage as injuries, including those accidents that resulted in fatalities.

The leading line of thought is that good or better performance leads to the decrease or absence of negative safety outcomes (Christian et al., 2009). As such, improved performance can be viewed as both a precursor of negative safety outcomes and as a goal in itself. Safety performance has been defined as those 'actions or behaviours that individuals exhibit in almost all jobs to promote the health and safety of workers, clients, the public, and the environment' (Burke, Sarpy, Tesluk, \& Smith-Crowe, 2002) and is considered to consist of two components: safety compliance and safety participation (e.g., Neal \& Griffin, 2002; Neal et al., 2000). Safety compliance refers to 'following safety procedures and carrying out work in a safe manner', whereas safety participation refers to 'helping coworkers, promoting the safety program within the workplace, demonstrating initiative, and putting effort into improving safety in the workplace' (Neal et al., 2000).

\subsubsection{Determinants}

A wide variety of possible precursors and determinants of safety have been studied. Examining the work environment, Bjerkan (2010) distinguishes between the physical work environment and the mental work environment. Whereas the physical work environment refers to tangible elements like machinery, the mental work environment refers to elements like job demands and working hours. Related elements that have attracted considerable attention from researchers are culture (e.g., Guldenmund, 2000) and climate (e.g., Zohar, 2010). 
Another topic of interest is the influence of (other) employees. Elements such as manager attitudes (e.g., Mullen, 2004), leadership styles (e.g., Kelloway, Mullen, \& Francis, 2006), and pressure exerted by colleagues (Choudhry, 2012) are all considered important influencers of behaviour. However, characteristics of individual employee such as age and experience, are considered important as well (e.g., Basha \& Maiti, 2013).

Finally, there are several external elements that might influence occupational safety. What are the effects of stakeholders, legislation, and external control bodies (e.g., Ko, Mendeloff, \& Gray, 2010)?

\subsection{Method}

To examine the foci of research to date, we conducted a systematic search in the occupational safety literature from 1980 to 2015. A systematic review of the literature is typically based on a 'detailed and comprehensive plan and search strategy derived a priori' in order to reduce bias (Uman, 2011). In contrast to a meta-analysis we do not strive to come to a 'single quantitative estimate or summary effect size' using statistical techniques (Uman, 2011). Instead, we aim to present an overview of topics addressed in both quantitative and qualitative research on occupational safety, and their general direction. This approach is similar to approaches in previous systematic reviews (e.g., Ahonen, Benavides, \& Benach, 2007; Kringos, Boerma, Hutchinson, Van der Zee, \& Groenewegen, 2010). Below, we will elaborate on our systematic selection process and analysis.

\subsubsection{Literature search}

Our aim was to capture as much of the available literature on occupational safety as possible. We therefore chose a literature search using broad search terms as a starting point, as opposed to citation networks which may result in overlooking new and less frequently cited literature. Our literature search was conducted using the following bibliographic databases: Scopus, Web of Science, PsycInfo, and Business Source Elite. We used combinations of keywords that emerged from the literature as key indicators of occupational safety: safety performance; safety participation; safety compliance; occupatio*; and employ* This resulted in a total of 27,527 records published between 1979 and 2015. 


\subsubsection{Article selection}

The further selection of articles was performed in steps, as depicted in Figure 2.1. Based on the available information in Endnote we removed duplicates, articles written in languages other than English, and -as a quality assurancenon-peer reviewed articles $(n=16,302)$. This step reduced the selection to 11,225 articles. Not all non-peer reviewed articles could be excluded based on the information available in Endnote. This resulted in the removal of articles matching this criterion during multiple phases of the selection process. Then, three consecutive steps were completed.

First, the first author evaluated the titles and marked articles that did not meet the following inclusion criteria: (1) describe safety in an occupational setting; (2) focus on interventions, determinants, or measurement of occupational safety; (3) conducted in the construction, warehouse, manufacturing, offshore, or petrochemical sector; (4) published in a peer-reviewed journal; and (5) be written in English. The four domains of construction, (offshore) petro chemistry, warehouses, and manufacturing were included based on a combination of elements. First, the construction and manufacturing sector combined accounted for more than a fifth of all fatal accidents that occurred in 2013 in the EU-28 (Eurostat, 2016). Second, the Dutch Inspectorate SZW mentions that the construction and chemistry are among those sectors where employees are subject to high health and safety risks (Inspectorate SZW, 2016), furthermore, the chemical sector has proven to be a domain where accidents can have a big environmental impact (e.g., Deepwater Horizon in 2010). Third, the domain of warehouses was included as employees here are subject to a high number of (mechanical) risks, such as forklifts and conveyors. Fourth, these four domains share a number of similarities that makes them relatively comparable: they represent highly technical environments with a number of occupational risks and are staffed with mostly blue-collar workers. Lastly, other well-studied areas are excluded as they represent highly specific risks (e.g., underground mining), require employees to be highly educated and trained (e.g., aviation), or mainly have a focus on the safety of others (e.g., hospitals).

A random sample of $10 \%$ of the articles was assessed for eligibility by the second author, which resulted in a substantial Cohen's Kappa for inter-coder reliability (.73). Based on the screening of titles, 6,558 articles were excluded and 4,667 articles remained. When there was any doubt or disagreement 
during this step, the article was retained for the next round of analysis. We repeated this process by reading the abstracts of the remaining articles. The Cohen's Kappa over the sample of abstracts $(n=474)$ was again substantial (.68). After exclusion of 2,600 articles based on abstract content, a sample of 2,067 articles remained. As the initial search was conducted during October 2014, we repeated our search during October 2015 so as to include all relevant articles published in the last months of 2014. This returned 24 additional articles, which underwent the same process of selection. From these 2,091 records we excluded any remaining grey and white literature $(n=324)$ and articles that were not published online $(n=614)$. The majority of the remaining articles were directly available for download. To retrieve the 222 articles that were published online but were unavailable to us through the subscriptions of University of Twente, we used a combination of ResearchGate and other university libraries. The full texts of the 1,153 articles were read and an additional 447 articles were excluded.

The remaining articles $(n=706)$ were read and assigned to one or multiple of three categories: research involving determinants of occupational safety ( $n=476)$; interventions research $(n=266)$; and research concerned with measurement tools $(n=141)$. The latter two groups were excluded from further analysis as they did not meet the current study focus. From the 476 articles focusing on determinants of occupational safety, 199 articles were excluded, mostly on the premise that they did not report on empirical data, or the reported data was not unique (e.g., editorials, reviews, meta-analysis). Inclusion of reviews and meta-analysis could distort the results, as the same data could be included twice.

The final step involved again reading the full texts of all remaining articles. All 277 remaining articles were read by one of the authors, who in the process marked potentially problematic articles. All of these marked articles were discussed by two of the authors. Based on the outcome of these discussions, a joint decision on whether or not to include these articles was made. This resulted in the exclusion of an additional 101 articles based on their full text, because they did not meet the inclusion criteria (e.g., dependent variable was not safety-related $(n=40)$; data-related issues, e.g., moderators, discrepancies between results and conclusion/discussion $(n=29)$; or analysis-related issues, e.g., solely factorial analyses, articles that presented relationships as being significant but had corresponding significance values that were above $.10(n=$ 32)). 
Records identified through database search $n=27,527$

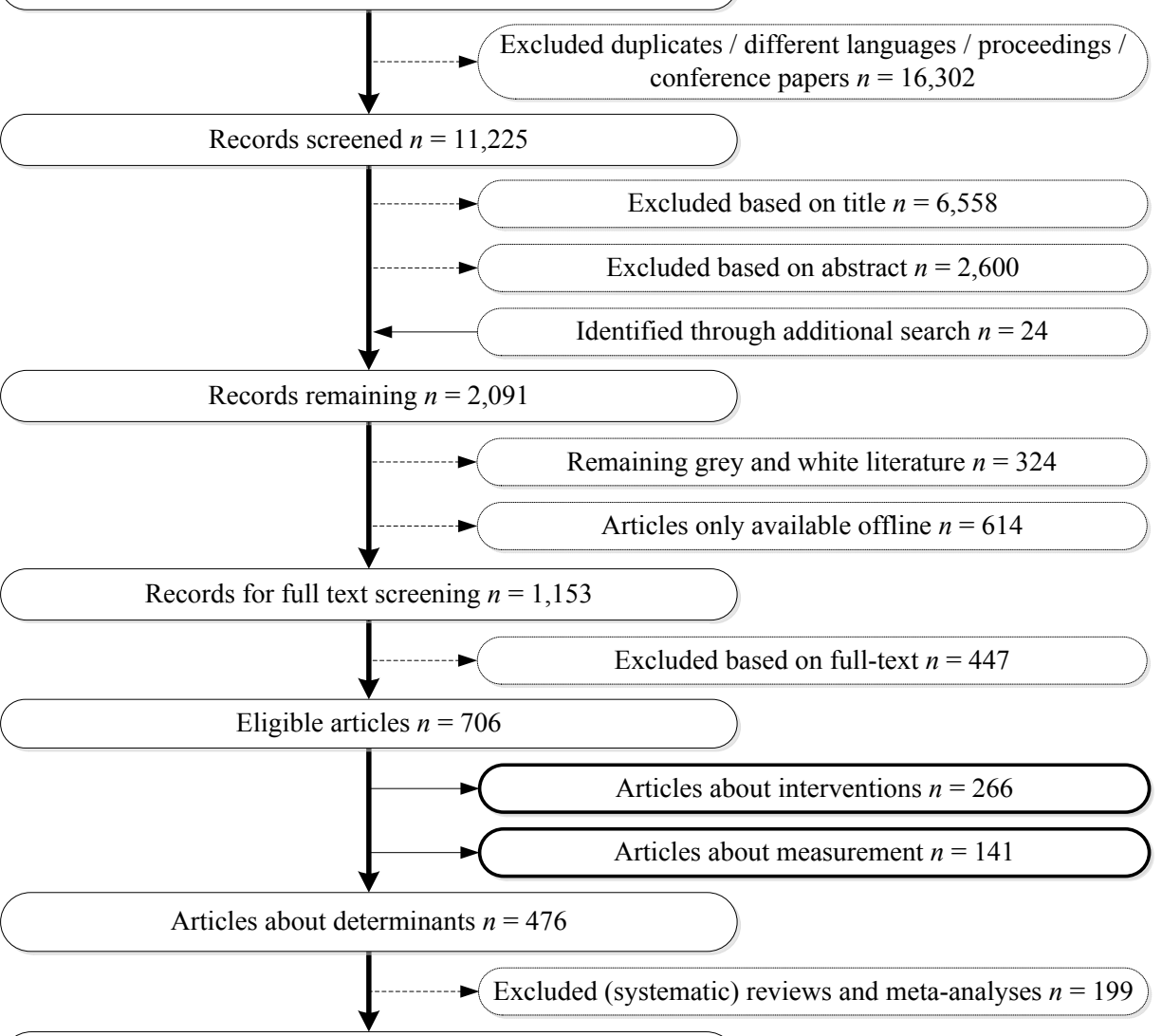

Full-text determinant articles assessed for eligibility $n=277$

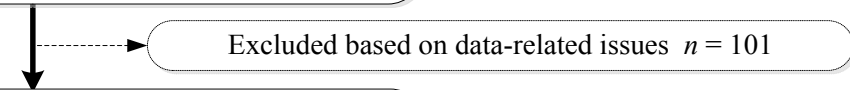

Articles included in review $n=176$

Unique variables extracted from articles $n=1,479$

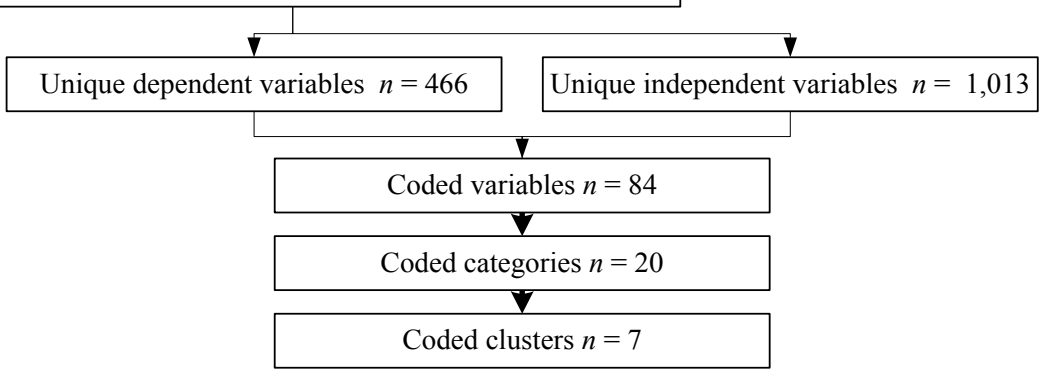

Figure 2.1. Flow diagram of study selection and extracted variables 


\subsubsection{Analysis}

For each article, we coded the following: a unique number, reference data according to the APA formatting style, type of industry in which the study was conducted, type of methodology used to conduct the research, number of participants that participated in the study, and aim of the study. Each article was dissected in terms of the (hypothesised) relationship between dependent and independent variables by one of the authors. Each relationship was assigned a row in a matrix, which described the relationship between two variables (e.g., the influence of safety climate on safety performance). For mediating relationships, we split the hypothesised relationship and created an additional line. For example, the relationship between variables $\mathrm{A}$ and $\mathrm{C}$, which was mediated by variable $B$, was transformed into two rows, in which one row was concerned with the relationship between variables $A$ and $B$, and the other row was concerned with the relationship between variables B and C. Finally, we assessed the effect each independent variable had on the dependent variable(s) (positive, negative, or inconclusive), and what the type of relationship was (correlation, regression, different). The indication about the type of relationship was not further included in the analysis. As such, numbers in the result section do not indicate causal relationships between variables. They do represent the number of times a positive, negative or insignificant relation between two variables was found in the analysed studies. A special type of relationship not anticipated at first was the (inverted) u-shaped relationship. These relationships are coded as '(-)U'. Relationships from quantitative studies were coded as inconclusive if the statistical significance was above the generally accepted threshold of .05. For relationships from qualitative studies we followed the conclusions presented in the article. Studies with significance levels above .10, in which the authors report on significant relationships, were excluded entirely from the dataset. From our corpus of 176 articles we initially extracted 2,202 relations. We excluded 96 relationships because they did not match the aim of our review (e.g., based on information about type of industry and regions) or involved moderating relationships and variables.

Within the 2,106 relationships, 1,479 unique variables names were identified, many of which were only unique in name but had similar meanings (e.g., occupational accident and work accident). To reduce clutter, we recoded variables with similar meanings into a new variable name. Using Microsoft 
Excel, we extracted 466 unique dependent and 1,013 unique independent variable values. Through a process of bottom-up coding two of the authors categorised these unique variables into 84 variables, categorised into 20 categories, which together formed seven clusters (see Figure 2.2). Some of the variables (3\%) proved difficult to categorise. Therefore, we omitted 29 dependent and 97 independent variables from the final analysis, resulting in a total of 1,946 fully coded relationships between variables in 174 articles.

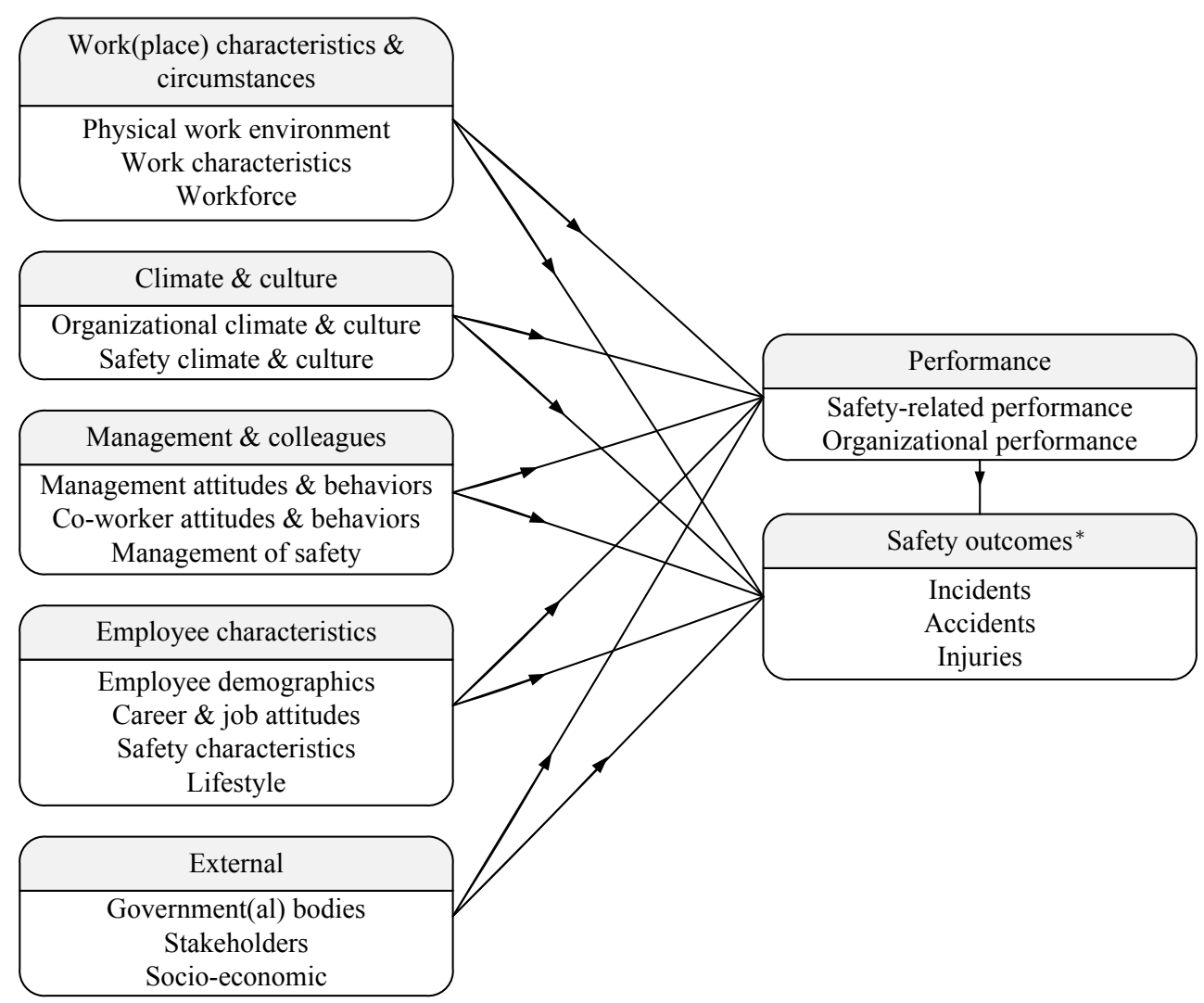

Figure 2.2. Clusters and their associated categories

*Note: the term 'Safety outcomes' refers to negative events in the form of incidents, accidents, or injuries. 


\subsection{Results}

We will begin by presenting the relationships between the seven clusters, followed by a more in-depth inspection of the results per cluster and their corresponding categories. An overview of all relationships between clusters is presented in Table 2.1. It is noteworthy that in terms of dependent (on the horizontal axis) and independent variables (on the vertical axis), the clusters of safety outcomes and performance together account for $63 \%$ of all dependent variables, while the clusters of performance, work(place) characteristics \& circumstances, climate \& culture, management \& colleagues, and employee demographics prevail (96\%) among the independent clusters. All corresponding references are included in Appendix A: Reference materials.

Table 2.1

Relationships between variables at the cluster level. Dependent clusters on the horizontal axis, independent clusters on the vertical axis.

\begin{tabular}{|c|c|c|c|c|c|c|c|c|}
\hline & $1 *$ & 2 & 3 & 4 & 5 & 6 & 7 & Total \\
\hline 1. Safety outcomes* & 13 & 3 & 4 & 3 & 7 & 2 & 1 & 33 \\
\hline 2. Performance & 54 & 28 & 10 & 7 & 7 & 11 & 1 & 118 \\
\hline $\begin{array}{l}\text { 3. Work(place) char. \& } \\
\text { circumstances }\end{array}$ & 254 & 112 & 61 & 14 & 37 & 46 & 1 & 525 \\
\hline 4. Climate \& culture & 23 & 40 & 8 & 7 & 9 & 22 & 1 & 110 \\
\hline $\begin{array}{l}\text { 5. Management \& } \\
\text { colleagues }\end{array}$ & 167 & 247 & 33 & 31 & 134 & 70 & 15 & 697 \\
\hline $\begin{array}{l}\text { 6. Employee } \\
\text { demographics }\end{array}$ & 174 & 103 & 29 & 27 & 37 & 52 & 1 & 423 \\
\hline 7. External & 12 & 5 & 2 & 18 & 3 & 0 & 0 & 40 \\
\hline Total & 697 & 538 & 147 & 107 & 234 & 203 & 20 & 1,946 \\
\hline
\end{tabular}

Note: This table also shows relationships within clusters, i.e., different variables grouped in the same cluster that have been related in included articles.

*The term 'Safety outcomes' refers to negative events in the form of incidents, accidents, or injuries. 
Below we will present the results on a more specific level per cluster, starting with the safety outcomes and performance of the more dependent clusters. However, to avoid presenting the same information twice, we will focus on the independent clusters: performance, work(place) characteristics \& circumstances, climate \& culture, management \& colleagues, employee demographics, and external. For each of these clusters we will start by presenting the number of relationships with the dependent clusters of safety outcomes and performance. This may cause that some relations between independent clusters are not described in more detail, for example the 18 relations between the external cluster and the climate \& culture cluster. The cluster descriptions will be followed by a summary in a table, providing the number of relationships and their nature per variable. Finally, we will highlight the most promising results per category.

\subsubsection{Safety outcomes}

The most prevalent dependent cluster turns out to be safety outcomes, accounting for over a third of all dependent variables. Within this cluster, we see that research is most concerned with the categories injuries and fatalities (56\%) and accidents (31\%), and less with incidents (13\%).

The most prominent cluster related to safety outcomes, with 254 relationships, was work(place) characteristics \& circumstances, followed by the cluster of employee demographics, which accounted for 174 relationships. The management \& colleagues cluster accounted for 167 relationships with safety outcomes, while the performance cluster accounted for only 54 relationships. Finally, the clusters climate \& culture and external together accounted for 35 of the relationships with safety outcomes. It is worth mentioning that over half of the relationships in the climate \& culture cluster are accounted for by the variable safety climate, with 13 relations.

\subsubsection{Performance}

The performance cluster plays both a dependent and an independent role in safety research. The relationships between the performance variables and the safety outcomes cluster are depicted in Table 2.2. Our review found 28 negative relationships between the category of safety-related performance and the cluster safety outcomes, 4 positive relationships, and 14 relationships that 
did not reach significance. The relationships in this cluster are centred around the variables safety performance, safety compliance, and safety participation, all of which fall into the safety-related performance category. However, Personal Protective Equipment (PPE) use was hardly mentioned.

Table 2.2

Relationships between performance and safety outcomes.

\begin{tabular}{lccc}
\hline Performance & \multicolumn{3}{c}{ Safety outcomes* } \\
\cline { 2 - 4 } & + & - & $n s$ \\
\hline Safety-related performance & 4 & 28 & 14 \\
Safety performance & 0 & 6 & 2 \\
PPE use & 0 & 4 & 0 \\
Safety compliance & 1 & 9 & 3 \\
Safety participation & 3 & 9 & 9 \\
Organizational performance & 3 & 3 & 2 \\
\hline Organizational performance & 0 & 0 & 0 \\
Financial performance & 1 & 0 & 1 \\
(Employee) work performance & 2 & 1 & 0 \\
Organizational quality performance & 0 & 1 & 0 \\
Production performance & 0 & 1 & 1 \\
Environmental performance & 0 & 0 & 0 \\
\hline Total & 7 & 31 & 16 \\
\hline
\end{tabular}

*Note: The term 'Safety outcomes' refers to negative events in the form of incidents, accidents, or injuries.

As a dependent variable, the category of safety-related performance accounts for the vast majority of relationships $(n=492)$, while the category organisational performance accounts for only 46 relationships. Performance is mostly studied in relation to the categories of management of safety, management attitudes \& behaviours, work characteristics, safety climate \& culture, and (employee) safety characteristics. Combined, these categories account for $66 \%$ of all relationships with performance. As depicted in Table 2.2, the category of safety-related performance indicates that most of the relations with safety outcomes are negative, with 28 out of a total 46 relationships resulting in fewer negative events in the form of incidents, 
accidents, or injuries. Out of the 46 relationships only four relationships result in more incidents, accident, or injuries. A total of 14 relationships are found to be non-significant. Below, the relationship between the more independent variables and the clusters of safety outcomes and performance will be further discussed.

\subsubsection{Work(place) characteristics \& circumstances}

The cluster of work(place) characteristics \& circumstances was related 254 times to safety outcomes and 112 times to performance. The cluster consisted of the categories: physical work environment, work characteristics, and workforce. The numbers of relationships between the variables in these categories, safety outcomes, and performance are depicted in Table 2.3.

\section{Physical work environment}

The category physical work environment accounted for a total of 98 relationships with the cluster negative safety outcomes and 29 relationships with the performance cluster. Looking at the individual variables in this category, one can see that the size of the company does not seem to matter in relation to either outcomes or performance. Workplace hazards clearly affect negative safety outcomes; however, the relationship with performance is less clear. In this category, (safety) equipment is studied most often, although the majority of the relationships fail to reach significance. However, the safety of equipment seems important for performance. The physical workplace (design) mainly seems to increase negative safety outcomes.

\section{Work characteristics}

The category work characteristics was related 137 times to negative safety outcomes and 52 times to performance. In this category, six variables stand out. The relationship between shift work and negative safety outcomes is heavily studied, although its direction remains unclear. Working hours are positively related to negative safety outcomes, as are job demands and production pressure. Job resources are negatively related to negative safety outcomes, and they are positively related to performance. The control employees have over their own work and safety is positively related to performance. 
Table 2.3

Relationships between work(place) characteristics \& circumstances, safety outcomes \& performance.

\begin{tabular}{|c|c|c|c|c|c|c|}
\hline \multirow{2}{*}{$\begin{array}{l}\text { Work(place) characteristics \& } \\
\text { circumstances }\end{array}$} & \multicolumn{3}{|c|}{ Safety outcomes* } & \multicolumn{3}{|c|}{ Performance } \\
\hline & + & - & $n s$ & + & - & $n s$ \\
\hline Physical work environment & 29 & 12 & 57 & 10 & 2 & 17 \\
\hline Company size & 3 & 2 & 0 & 3 & 1 & 8 \\
\hline Workplace hazards & 7 & 0 & 7 & 1 & 0 & 2 \\
\hline (safety) equipment & 4 & 5 & 28 & 2 & 0 & 2 \\
\hline Safety of equipment & 5 & 3 & 11 & 4 & 0 & 1 \\
\hline Physical workplace (design) & 10 & 2 & 11 & 0 & 1 & 4 \\
\hline Work characteristics & 52 & 23 & 62 & 20 & 11 & 21 \\
\hline Work characteristics & 1 & 0 & 4 & 0 & 0 & 1 \\
\hline Perceived work(place) safety & 0 & 0 & 2 & 0 & 4 & 1 \\
\hline Goal setting & 1 & 2 & 2 & 3 & 0 & 2 \\
\hline Stress & 3 & 0 & 1 & 0 & 0 & 0 \\
\hline Shifts & 5 & 5 & 14 & 0 & 1 & 0 \\
\hline Working hours & 11 & 0 & 1 & 0 & 0 & 1 \\
\hline Job demands & 11 & 0 & 9 & 1 & 0 & 2 \\
\hline Job resources & 2 & 13 & 10 & 8 & 0 & 5 \\
\hline Production (pressure) & 16 & 2 & 12 & 1 & 6 & 8 \\
\hline Task clarity & 1 & 0 & 1 & 1 & 0 & 1 \\
\hline (safety) control & 1 & 1 & 6 & 6 & 0 & 0 \\
\hline Workforce & 6 & 3 & 10 & 4 & 2 & 25 \\
\hline Contract type & 0 & 0 & 0 & 0 & 1 & 8 \\
\hline Job level & 0 & 2 & 1 & 0 & 1 & 4 \\
\hline Workforce quantity & 0 & 1 & 4 & 0 & 0 & 0 \\
\hline Workforce composition & 4 & 0 & 1 & 0 & 0 & 7 \\
\hline Unions & 1 & 0 & 3 & 3 & 0 & 6 \\
\hline HR & 1 & 0 & 1 & 1 & 0 & 0 \\
\hline Total & 87 & 38 & 129 & 34 & 15 & 63 \\
\hline
\end{tabular}

*Note: The term 'Safety outcomes' refers to negative events in the form of incidents, accidents, or injuries. 


\section{Workforce}

The category workforce has been related 19 times to safety outcomes and 31 times to performance. The majority of these relationships are not significant. Contract type does not seem to be related to performance, while relationships with outcomes are lacking overall. Workforce composition is mostly positively related to outcomes, while it does not affect performance. The majority of relationships among unions, outcomes, and performance are not significant.

\subsubsection{Climate \& culture}

A total of 23 relationships were found between the cluster climate \& culture and safety outcomes, and 40 relationships were found between climate $\&$ culture and performance. The cluster consists of the categories organisational climate \& culture and safety climate \& culture. The number of relationships between the variables in each category and the clusters safety outcomes and performance are depicted in Table 2.4.

Looking at the category organisational climate \& culture, most relationships are found between climate and outcomes $(n=9)$. While not well established, most of these relationships are negative. With a total of 36 relationships,

Table 2.4

Relationships between climate \& culture, safety outcomes \& performance.

\begin{tabular}{lcccccccc}
\hline Safety climate \& culture & \multicolumn{3}{c}{ Safety outcomes* } & & \multicolumn{3}{c}{ Performance } \\
\cline { 2 - 4 } & + & - & $n s$ & & + & - & $n s$ \\
\hline Organisational climate \& culture & 0 & 5 & 4 & & 2 & 0 & 0 \\
Organisational climate & 0 & 5 & 4 & & 1 & 0 & 0 \\
Organisational culture & 0 & 0 & 0 & & 1 & 0 & 0 \\
\hline Safety climate \& culture & 2 & 6 & 6 & & 29 & 1 & 8 \\
Safety climate & 1 & 6 & 6 & & 25 & 1 & 7 \\
Safety culture & 1 & 0 & 0 & & 4 & 0 & 1 \\
\hline Total & 2 & 11 & 10 & & 31 & 1 & 8 \\
\hline
\end{tabular}

*Note: The term 'Safety outcomes' refers to negative events in the form of incidents, accidents, or injuries. 
safety climate is by far the most studied variable in the safety climate \& culture category. The 25 positive relationships between safety climate and performance particularly stand out.

\subsubsection{Management \& colleagues}

The cluster management \& colleagues was related to the cluster safety outcomes 167 times, and with the performance cluster 247 times. The management \& colleagues cluster consisted of the categories management attitudes \& behaviours, co-worker attitudes \& behaviours, and management of safety. The numbers of relationships between the variables in each category, safety outcomes, and performance are depicted in Table 2.5.

\section{Management attitudes \& behaviours}

The management attitudes \& behaviours category was related 43 times to the safety outcomes cluster, and 80 times to the performance cluster. Two variables in this category stand out. Concerning the relationships between leadership (style) and outcomes, it is striking that most of them are not significant, while the majority of the relationships with performance are positive. The relationship between management attitudes and performance is studied intensively, and all of the relationships that reach significance are positive.

\section{Co-worker attitudes \& behaviours}

The category co-worker attitudes \& behaviours was related 12 times to safety outcomes, and 17 times to performance. Interestingly, no positive relationships were found between the category co-worker attitudes \& behaviours and outcomes, and no negative relationships with performance were found.

\section{Management of safety}

The majority of relationships in this cluster could be attributed to the management of safety category, as it was related 112 times to safety outcomes, and 150 times to performance. Six variables stand out in terms of their numbers of relations. Inspections are predominately negatively related to 
Table 2.5

Relationships between management \& colleagues, safety outcomes \& performance.

\begin{tabular}{|c|c|c|c|c|c|c|}
\hline \multirow[t]{2}{*}{ Management \& colleagues } & \multicolumn{3}{|c|}{ Safety outcomes* } & \multicolumn{3}{|c|}{ Performance } \\
\hline & + & - & $n s$ & + & - & $n s$ \\
\hline Management attitudes \& behaviours & 4 & 15 & 24 & 44 & 1 & 35 \\
\hline Leadership (style) & 0 & 3 & 14 & 14 & 1 & 8 \\
\hline Management attitudes & 2 & 5 & 5 & 23 & 0 & 21 \\
\hline Management behaviours & 2 & 3 & 4 & 5 & 0 & 3 \\
\hline $\begin{array}{l}\text { Safety importance for } \\
\text { management }\end{array}$ & 0 & 4 & 1 & 2 & 0 & 3 \\
\hline Co-worker attitudes \& behaviours & 0 & 4 & 8 & 8 & 0 & 9 \\
\hline Co-worker attitudes & 0 & 2 & 3 & 5 & 0 & 6 \\
\hline Co-worker behaviours & 0 & 2 & 5 & 3 & 0 & 3 \\
\hline Management of safety & 12 & 39 & 61 & 85 & 7 & 58 \\
\hline Management of safety & 2 & 2 & 0 & 3 & 0 & 2 \\
\hline Inspections & 2 & 7 & 5 & 8 & 2 & 6 \\
\hline $\begin{array}{l}\text { Accident analysis \& record } \\
\text { keeping }\end{array}$ & 1 & 3 & 2 & 3 & 0 & 5 \\
\hline Safety representation & 3 & 1 & 16 & 2 & 0 & 4 \\
\hline Sanctions & 0 & 1 & 0 & 2 & 2 & 1 \\
\hline Rewards & 1 & 5 & 7 & 3 & 0 & 9 \\
\hline Accident reducing measures & 0 & 5 & 8 & 6 & 1 & 3 \\
\hline Training & 1 & 5 & 11 & 15 & 1 & 10 \\
\hline (safety) communication & 2 & 8 & 6 & 28 & 1 & 6 \\
\hline Safety policies \& procedures & 0 & 1 & 1 & 10 & 0 & 4 \\
\hline (safety) meetings \& activities & 0 & 1 & 5 & 5 & 0 & 8 \\
\hline Total & 16 & 58 & 93 & 137 & 8 & 102 \\
\hline
\end{tabular}

*Note: The term 'Safety outcomes' refers to negative events in the form of incidents, accidents, or injuries. 
outcomes, while their relationship with performance is mostly positive. Surprisingly, most relationships between safety representation and outcomes are not significant. The majority of the relationships among rewards, outcomes and performance are not significant. Accident reducing measures are negatively related to outcomes and positively related to performance. Two of the most represented variables are training and (safety) communication. Both are mostly positively related to performance.

\subsubsection{Employee demographics}

Our review found a total of 174 relationships between the cluster employee demographics and safety outcomes, and it found 103 relationships with performance. This cluster consists of the categories employee demographics, career \& job attitudes, safety characteristics, and lifestyle. The number of relationships between the variables in each category, safety outcomes, and performance are depicted in Table 2.6.

\section{Employee demographics}

The employee demographics category was related 55 times to the safety outcomes cluster and 30 times to the performance cluster. The relationship between age and outcomes has been heavily studied. Concerning gender, the results for both outcomes and performance are inconclusive, with no clear majority of positive or negative relations. The relationship between disabilities and performance seems to be understudied, as no research in our sample has discussed the relationship between the two variables.

\section{Career \& job attitudes}

The category career \& job attitudes was related 72 times to safety outcomes and 28 times to performance. The two variables tenure/experience and trust are frequently studied in relation to both outcomes and performance. While tenure/experience is mostly negatively related to outcomes, and mostly positively related to performance, it should be noted that, again, a U-shaped relationship was found between tenure/experience and performance in one study. For the relationship between trust and outcomes, the results seem inconclusive as both positive and negative results are found, while the relationship between trust and performance is mostly positive. 
Table 2.6

Relationships between employee demographics, safety outcomes \& performance.

\begin{tabular}{lcccccccc}
\hline Employee demographics & \multicolumn{3}{c}{ Safety outcomes* } & & \multicolumn{3}{c}{ Performance } \\
\cline { 2 - 4 } \cline { 3 - 4 } & + & - & $n s$ & & + & - & $n s$ \\
\hline Employee demographics & 14 & 24 & 17 & & 15 & 2 & 13 \\
Age & 7 & $8^{* *}$ & 12 & & 3 & 1 & 6 \\
Gender (female) & 3 & 3 & 1 & & 0 & 0 & 2 \\
Education & 2 & 4 & 1 & & 3 & 1 & 3 \\
Disabilities & 2 & 0 & 2 & & 0 & 0 & 0 \\
Psychophysical states & 0 & 9 & 1 & & 9 & 0 & 2 \\
\hline Career \& job attitudes & 15 & 29 & 28 & & 16 & 1 & 11 \\
Tenure/experience & 9 & 18 & 16 & & $6 * * *$ & 1 & 7 \\
Employee work attitudes & 0 & 3 & 3 & & 4 & 0 & 1 \\
Trust & 6 & 8 & 9 & & 6 & 0 & 3 \\
\hline Safety characteristics & 5 & 3 & 12 & & 26 & 2 & 9 \\
Employee safety attitudes & 1 & 1 & 4 & & 2 & 0 & 1 \\
(safety) motivation & 1 & 0 & 4 & & 9 & 1 & 5 \\
Safety knowledge (sharing) & 2 & 1 & 2 & & 15 & 1 & 3 \\
Responsibility & 1 & 1 & 2 & & 0 & 0 \\
\hline Lifestyle & 8 & 5 & 14 & & 4 & 2 & 2 \\
Work-life balance & 0 & 3 & 2 & & 2 & 0 & 0 \\
Marital status & 0 & 0 & 3 & 1 & 0 & 0 \\
Children & 1 & 0 & 0 & 0 & 0 & 2 \\
Lifestyle & 2 & 2 & 7 & & 0 & 0 \\
Lifestyle disorders \& & 5 & 0 & 2 & & 0 & 2 & 0 \\
substance abuse & 42 & 61 & 71 & 61 & 7 & 35 \\
\hline Total & & & & & &
\end{tabular}

*Note: The term 'Safety outcomes' refers to negative events in the form of incidents, accidents, or injuries.

**Age 1 time -U-shaped relationship with outcomes

***Tenure/experience 1 time U-shaped relationship with performance 


\section{Safety characteristics}

The category safety characteristics was related 20 times to safety outcomes and 37 times to performance. The focus in this category is on the relationship between safety knowledge (sharing) and performance. No fewer than 15 positive relationships were found, indicating that the more safety knowledge within the organisation, the better the performance. The same holds for (safety) motivation, which was positively related to performance nine times. It is striking that we failed to find any studies relating responsibility and performance.

\section{Lifestyle}

Just over $10 \%$ of all relationships in this cluster were accounted for by the category lifestyle, making it an understudied topic. This category was related 27 times to safety outcomes, and just 8 times to performance.

\subsubsection{External}

Our review yielded only 12 relationships between the external cluster and safety outcomes, and a mere five relationships with performance. The external cluster consists of the categories government(al) bodies, stakeholders, and socio-economic. The number of relationships between the variables in each category, safety outcomes, and performance are depicted in Table 2.7.

The category government(al) bodies was related three times to safety outcomes and three times to performance. Laws \& legislation have not been successfully related to performance and only once to safety outcomes. The category stakeholders was not related to either safety outcomes or performance. As both variables in the stakeholders category weren't linked to either safety outcomes or performance it is of added value to include these in Table 2.7. However, the overall analysis revealed that these variables were present in the literature, as the variable client involvement was related 17 times to the variable safety climate, and the variables injury rate, safety participation, and safety climate were each related one time to the variable customer satisfaction. For both the sake of completeness, and uncovering it as a potential gap in research we included these variables nevertheless. Lastly, the category of socio-economic variables is the most prominent of the three. However, the focus is rather one-sided in favour of outcomes. 
The most prevalent results are summarised in the figure below, where categories and the number of relationships with performance and outcomes are depicted (Figure 2.3).

Table 2.7

Relationships between external, safety outcomes \& performance.

\begin{tabular}{lcccccccc}
\hline \multirow{2}{*}{ External } & \multicolumn{3}{c}{ Safety outcomes } & & \multicolumn{3}{c}{ Performance } \\
\cline { 2 - 4 } \cline { 6 - 8 } & + & - & $n s$ & & + & - & $n s$ \\
\hline Government(al) bodies & 2 & 1 & 0 & & 0 & 0 & 3 \\
$\quad$ Law \& legislation & 1 & 0 & 0 & & 0 & 0 & 3 \\
$\quad$ Government(al) bodies & 1 & 1 & 0 & & 0 & 0 & 0 \\
\hline Stakeholders & 0 & 0 & 0 & & 0 & 0 & 0 \\
$\quad$ Client involvement & 0 & 0 & 0 & & 0 & 0 & 0 \\
Customer satisfaction & 0 & 0 & 0 & & 0 & 0 & 0 \\
Socio-economic & 3 & 4 & 2 & & 1 & 0 & 1 \\
Economic factors & 3 & 2 & 0 & & 1 & 0 & 1 \\
Insurance \& costs of safety & 0 & 2 & 2 & & 0 & 0 & 0 \\
\hline Total & 5 & 5 & 2 & & 1 & 0 & 4 \\
\hline
\end{tabular}

*Note: The term 'Safety outcomes' refers to negative events in the form of incidents, accidents, or injuries.

\subsection{Discussion}

The results indicate that the majority of published literature on occupational health and safety from 1980 to 2015 has focused on safety outcomes and performance. The studied relationships were not equally distributed among the independent clusters with predictors. The majority of research focused on the effects of management \& colleagues, work(place) characteristics and circumstances, and employee demographics on safety outcomes and performance, and less on climate \& culture and external variables. In the three sections that follow we will discuss the most important findings of this study. First, we will discuss those variables about which there is some degree of consensus. Second, we will discuss those variables whose effects are cause for debate. Third, we will discuss possible gaps that our review has brought to light. 

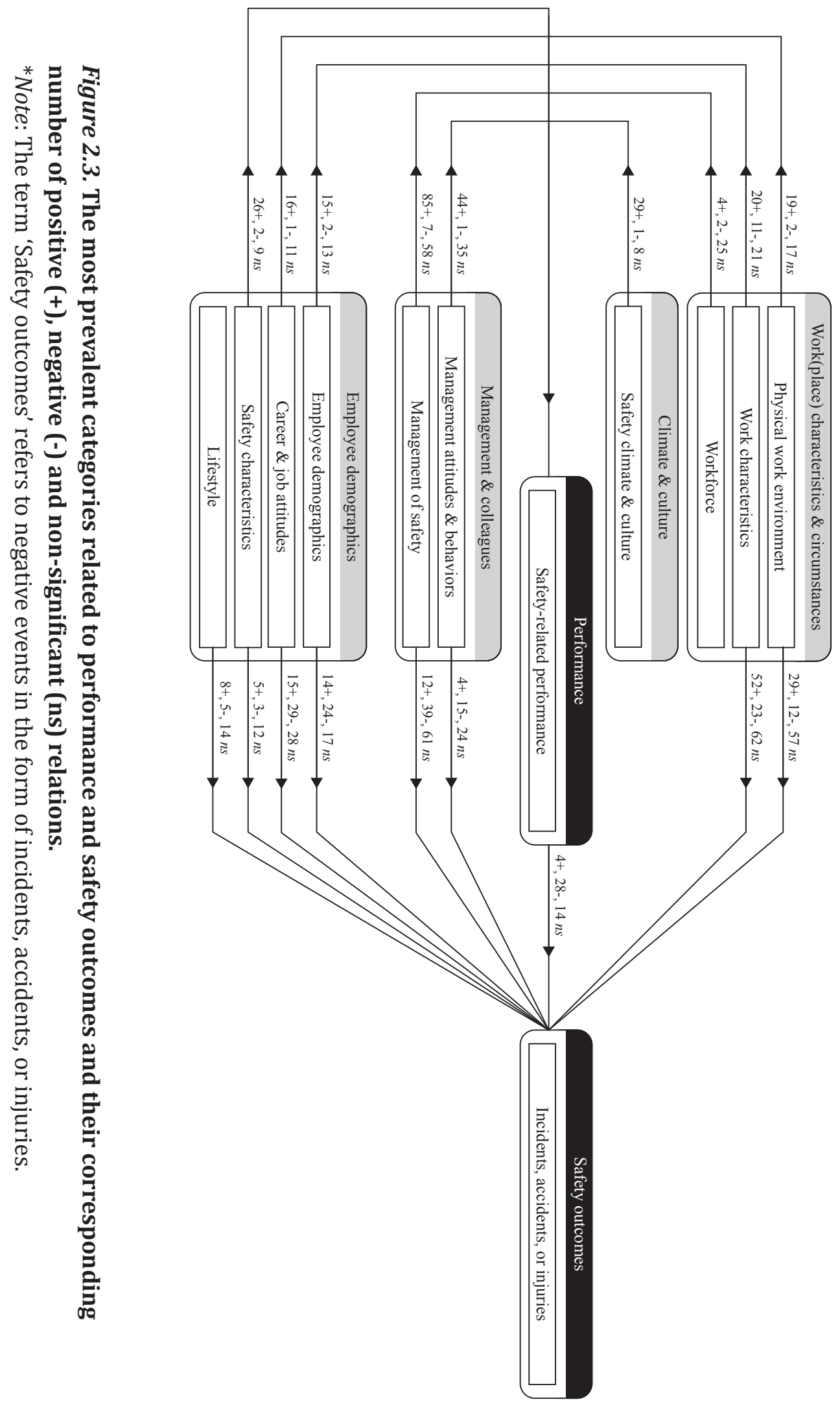


\subsubsection{Consensus in safety research}

We have set the threshold for consensus as a two-thirds majority supporting one of the three possible outcomes: positive relationship, negative relationship, or non-significant relationship. Here we will briefly discuss those variables that have been investigated more than five times. We will focus particularly on those variables on which organisations -to some extent- can exert influence.

The majority of relationships between safety performance and outcomes were negative, indicating that more or better safety performance is related to fewer incidents, accidents, and injuries. This finding is in agreement with the meta-analysis by Christian et al. (2009). The same holds for the relationships between safety compliance and outcomes, which is in line with research from other fields (e.g., Neal et al., 2000). Working hours and lifestyle disorders were positively related to negative safety outcomes. This positive relationship indicates an increase in incidents, accidents, or injuries if the variables increase or are more present. These relationships are not surprising, as it can be expected that more or longer working hours will result in a higher chance of being affected by negative safety outcomes -as is underlined by Wagstaff and Lie (2011)- as well as in the negative nature of lifestyle disorders (e.g., Melamed \& Oksenberg, 2002).

The remaining relationships were mostly related to performance, and all relationship directions were positive. Organisations that want to improve performance should therefore focus on (safety) control, safety climate, management behaviours, (safety) communication, safety policies \& procedures, psychophysical states, and safety knowledge (sharing). The category psychophysical states was also negatively related to safety outcomes, indicating a reduction in incidents, accidents, or injuries.

\subsubsection{Debates in safety research}

Various variables show inconsistent or contradictory effects in safety research and do not reveal a consensus. Our review yielded 36 variables related to safety outcomes or performance that were studied more than five times but did not result in clear findings. The majority of these relationships are subject to debate, as the results of the relevant studies are either positive or negative and non-significant. 
The relationship between safety participation and outcomes is not as clear as that between safety performance and safety compliance. Most studies either found a negative relationship - indicating that more or higher safety participation leads to fewer incidents, accidents or injuries - or a nonsignificant relationship.

Looking at the cluster of work(place) characteristics \& circumstances, workplace hazards tend to be positively related to safety outcomes, although an equal number of relationships failed to reach significance. The same is clear for the relationship between physical workplace (design) and outcomes. The relationship between the safety of equipment and outcomes is mostly nonsignificant. One possible explanation is offered by Saari (1982), who found that although machine guarding initially reduced the number of accidents, it did not overcome accidents that occurred during maintenance and repair tasks, 'when machine guarding is not easily possible' (Saari, 1982, p. 91).

Looking at the effects of shifts on safety outcomes, the results are mostly non-significant. However, we also found an equal number of positive and negative relationships. A more in-depth comparison of the different types of shifts might clarify the nature of this relationship, as research found that rotating shifts have more adverse effects than permanent night shifts (Muecke, 2005). Ignoring the number of relationships that failed to reach significance, there seem to be fairly strong positive and negative relationships between job demands \& resources and outcomes, and between job resources and performance. It would be useful to further explore the usability of job demands and resources in addressing occupational safety, as its effects reach beyond safety outcomes and performance (Bakker \& Demerouti, 2007). The relationships among production (pressure), safety outcomes and performance are inconclusive. Neglecting the non-significant results, production (pressure) seems to increase negative safety outcomes and reduce performance.

Although the link between safety climate and performance is well established, this is not the case for safety outcomes. The results illustrate a negative relationship with safety outcomes, although an equal number of nonsignificant relationships were found. This ambiguity is in agreement with research in other fields, where researchers have found not only that the safety climate might act as a mediator between more distal elements and safety 
outcomes (e.g., Wallace, Popp, \& Mondore, 2006) but also that the mediating role of the safety climate is very limited (e.g., DeJoy et al., 2004).

In the management \& colleagues cluster, many relationships are subject to debate. Considering the influence of management attitudes and behaviours, the field could definitely benefit from additional research. Whereas the relationship with safety outcomes is truly unclear, the findings regarding performance, leadership (style) and management attitudes tend to be more unanimous and indicate favourable influences on performance. The influence of co-workers on performance is almost equally distributed between positive and non-significant relations. Based on our results, it can be expected that inspections are good for the prevention of negative safety outcomes and have a positive influence on performance, although there are some contradictory findings. The effect of rewards on negative safety outcomes is mostly insignificant, in line with the relationship between rewards and performance. This might be explained by the very strict conditions under which rewards are effective (e.g., Schwartz \& Sharpe, 2010). Surprisingly, accident-reducing measures are more likely to be associated with good performance than with the prevention of negative safety outcomes.

Although our results illustrate that there is no clear consensus on the relationship between training and performance, training tends to have a positive effect on performance. Whereas the relationship between (safety) communication and performance was rather clear, this was not the case for safety communication's relationship with safety outcomes. The effect of (safety) meetings \& activities on performance is unclear, as most of the found relationships did not reach significance. The remaining relationships in our review indicate a positive effect of (safety) meetings \& activities on performance.

Last, we will discuss the cluster of employee characteristics. One study might explain the seemingly inconclusive results between age and negative safety outcomes and performance. Siu et al. (2003) found that 'age has a curvilinear effect on occupational injuries in which the frequency of injury increases with age first, then declines.' Gender does not seem to affect safety outcomes, as there are as many positive as negative relationships. Results concerning the variable tenure / experience, and its relationships with safety outcomes and performance, seem inconclusive at first. However, similar to the age 
variable relationship, Siu et al. (2003) found a curvilinear relationship with performance, indicating that performance would decrease at first and then increase with more tenure / experience. However, this relationship has not yet been examined for safety outcomes, so the lack of clarity for this relationship remains. Future research should further examine the relationship between (safety) motivation and performance, which, based on our results, is expected to be positive.

\subsubsection{Gaps}

Our review brought to light 21 variables that have not, or have hardly, been studied in relation to either negative safety outcomes or performance. Two variables have not been directly related to either outcomes or performance: client involvement and customer satisfaction. Client involvement has been successfully related to safety climate (Votano \& Sunindijo, 2014). Future research should examine the possible effects that client involvement could have on performance and safety outcomes. Customer satisfaction might have an indirect influence but is more likely to be considered a dependent variable. We found that customer satisfaction was studied as a dependent variable in relation to injury rates, safety participation, and safety climate, with mixed results.

The direct relationships between five variables and safety outcomes appear to lack investigation. These variables are contract type, safety culture, sanctions, children, and law \& legislation. Of these five, only the variables contract type and sanctions are directly controllable by the organisation, although it is strange that the relationship between safety culture and safety outcomes and the relationship between law \& legislation and safety outcomes lack research. Sanctions seems to be one of the most promising variables, as it has been successfully related to occupational safety through safety performance (e.g., Hasan \& Jha, 2013).

The relationship between performance and 14 variables appears to have been scarcely investigated. These variables are organisational culture, work characteristics, stress, shifts, working hours, workforce quantity, HR, organisational climate, disabilities, responsibility, marital status, lifestyle, government(al) bodies, and insurance $\&$ costs of safety. Of these variables, work characteristics, working hours, and responsibility seem to be the most 
promising leads for future research as they can be dealt with to some extent by the organisation. The concept of responsibility seems particularly interesting, as previous research in the education field associated increased responsibility with increased levels of self-confidence and perceived capability (Clouder, 2009).

\subsubsection{Limitations}

The conducted literature review has some limitations that will be discussed in relation to the steps conducted in this study. With regard to the literature search and selection of articles some potential limitations need to be addressed. In finding appropriate literature, the choice was made to use online databases only. This might have resulted in a shifted distribution of articles over the years, due to older publications that might not be digitally available. On the other hand, and this was our main rationale to use online publications only, it increases the replicability and controllability of this study as online articles are easily accessible to other researchers. Our literature search did not include keywords like 'injury' and 'injuries', that might be common in this or adjoining fields. This deliberate choice was made in order to direct the focus towards determinants of safety, instead of the wide range of potential occupational safety outcomes such as injuries, near misses, accidents, incidents, or lost-time rates. Due to the sheer number of publications it was not feasible to include each and every study that was conducted across the four industries of interest. However, the selection method applied in this review was thorough and transparent, and it provides other scholars with the opportunity to perform complementary reviews. Limiting the included literature to four specific domains lead to the absence of certain relationships that have been established in other domains. The gaps identified in our research are purely based on gaps present in the included literature. With regard to the analysis of the data, a potential limitation is related to the lack of interrater reliability in selecting articles based on their full text, and the extraction of individual relationships from the articles. Instead, potentially problematic cases were discussed and decided upon by two of the authors together. The same holds true for the recoding of extracted variables, as this whole process was done by two of the authors together. In the process of recoding variables from the bottom up, we have exchanged some depth and accuracy in favour of clarity and concision. In analysing the data, differences in power and magnitude across different studies were not taken into account. A 
study performed with as few as ten participants has the same 'power' assigned to it as a study with over one thousand participants. Although this might have implications when looking for specific solutions, it does capture the overall orientation in the safety literature. Our method of analysis did not allow for the inclusion of moderating variables or relationships. Although this might have some impact on the results, we believe the impact to be small due to the small number of moderating variables that were excluded. Finally, although we report the number of positive, negative, and non-significant relationships found, it must be noted that these are underestimates of the total number of relationships in the literature. In particular, the latter -non-significant relations- will suffer from both underreporting and underpublishing.

\subsubsection{Practical applications}

The results of this systematic review provide several practical leads for safety practitioners. First, there is an increasing need among practitioners for grounded knowledge about the field of occupational safety. Our results provide practitioners with a comprehensive overview of the elements present in the literature, which serves as good starting point for getting up to speed with the field. Second, the comprehensive overview of the elements and the nature of their relation with negative safety outcomes and performance, provides practitioners with a number of leads useful in the development and evaluation of interventions aimed at improving occupational safety.

\subsection{Conclusion}

This systematic review mapped scientific research from 1980 to 2015 in the domains of construction, (offshore) petro chemistry, warehouses, and manufacturing. In doing so, it showed that research is focused on improving safety outcomes and performance through variables associated with work(place) characteristics \& circumstances, climate \& culture, management \& colleagues, employee demographics, and external factors. 


\section{Acknowledgements}

We would like to thank Saskia Kelders, PhD, from the Department of Psychology, Health and Technology for her advice during the process of writing and designing this study. This research did not receive any specific grant from funding agencies in the public, commercial, or not-for-profit sectors. 


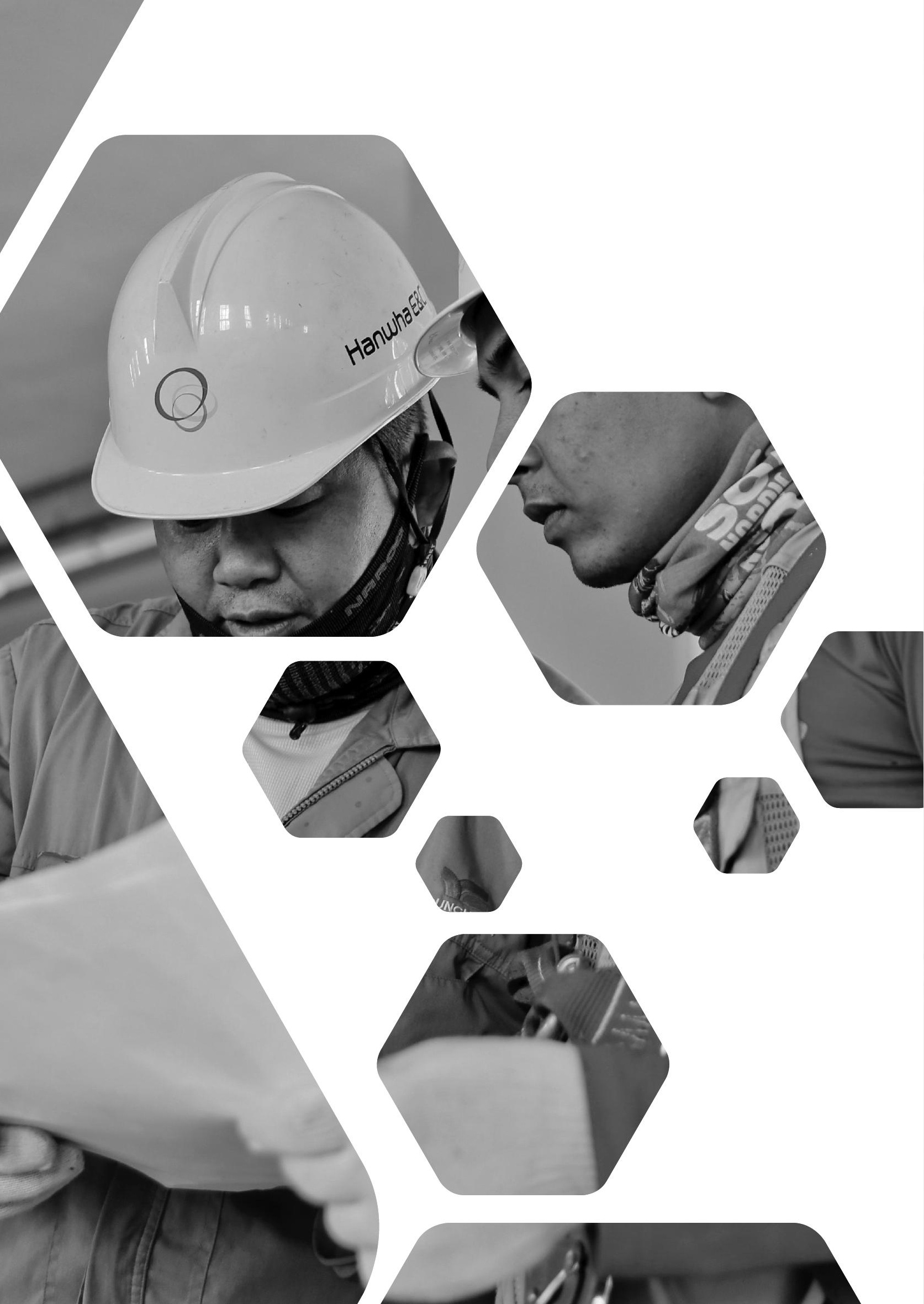


Chapter 3

\section{Supervisors' beliefs and attitudes towards occupational safety}

'Let's manage safety recognizing how humans are and stop managing safety the way we wish humans were.'

Alan Quilley

Cornelissen, P.A., Van Hoof, J.J., \& De Jong, M.D.T. 


\subsection{Introduction}

Occupational accidents and (fatal) injuries remain a major and persistent problem, as illustrated by the estimated 374 million occupational accidents and 2.7 million work-related deaths annually worldwide ("Safety and health at work", n.d.). Researchers and safety practitioners have attempted to reduce the number and impact of such events through both engineering and social approaches (Törner \& Poussette, 2009). In doing so, most efforts are aimed at the group that is most at risk: employees (Rundmo \& Hale, 2003). Improving employee safety through the use of interventions and campaigns aimed at increasing their knowledge, motivation, and ability have proven effective (Christian et al., 2009). However, within organisational settings, supervisors play an important role in creating a safe workplace (Barling et al., 2002; Rundmo \& Hale, 2003; Thompson, Hilton, \& Witt, 1998; Zohar \& Luria, 2003) as supervisors become increasingly critical for 'delegating job tasks, managing subordinate performance, and juggling competing demands for productivity, quality, and safety' (Michael et al., 2006, p. 469).

The role of supervisors has been extensively studied in the occupational safety context. The majority of this research is centred on supervisors' interaction with subordinates (e.g., Hofmann, Morgeson, \& Gerras, 2003; Michael et al., 2006), differences in leadership styles (e.g., Clarke, 2013; Kelloway et al., 2006), and supervisor attitudes and commitment as perceived by employees (e.g., Siu et al., 2003; Zohar, 1980). Despite their importance for behavioural intentions and safety behaviour (Madden, Ellen, \& Ajzen, 1992; Rundmo \& Hale, 2003), extensive research on supervisors' own attitudes is lacking. The researchers that have studied supervisors' perceptions of and attitudes towards occupational safety have mainly used a quantitative approach (Rundmo \& Hale, 2003), focused on a specific managerial level (O'Dea \& Flin, 2001), or on a limited set of safety-related elements, such as safety climate and safety culture (Törner \& Pousette, 2009). Notwithstanding the contributions made by these studies to the occupational safety literature, they do not take into account the range of the managerial spectrum and the wide variety of safety-related factors that may be influenced by supervisors' attitudes and do not allow for an in-depth exploration of these attitudes, let alone the emergence of new and unexpected factors. Therefore, the aim of this study is to extend the current, predominantly instrumental, view of supervisors in occupational safety research using semi-structured 
interviews. Supervisors' perceptions, beliefs, attitudes, and self-reported behaviours towards occupational safety are explored. Increased insights and understanding of supervisors' safety beliefs and attitudes could further the current understanding of management decisions, help to explain the (lack of) success of certain safety management instruments, and eventually create safer workplaces. To do so, the following main research question was formulated:

What perceptions, beliefs, and attitudes do supervisors hold regarding occupational safety, and how do such perceptions, beliefs, and attitudes influence the management of safety?

This research was conducted in the railway construction and maintenance sector. Within this sector, much of the work is performed at night, at varying and remote locations, and with limited time windows for maintenance and great time pressure for construction projects (Turner, Chmiel, \& Walls, 2005), making the role of supervisors difficult due to high levels of segregation (in time and space) between them and their subordinates. The railway construction and maintenance sector struggles with occupational safety, as evidenced by inspection results from the Human Environment and Transport Inspectorate ("Veiligheid bij werk aan spoor", 2017). These characteristics make it a perfect starting point for the exploration of supervisors' safety attitudes and beliefs.

\subsection{Theoretical framework}

Below, an overview is provided of the literature, centred on three topics. First, the previous literature on the role of occupational safety in work contexts is discussed. This is followed by the literature on supervisors' safety beliefs and their attitudes towards safety management instruments. For every topic, a separate sub-research question is formulated.

\subsubsection{The role of occupational safety in the work context}

The perceived role of occupational safety in supervisors' work is an interesting viewpoint to consider, especially as supervisors' views may differ from employee views (Eakin, 1992; Mohamed, 2003). In exploring supervisors' views of occupational safety, several variables can be taken into 
account. Supervisors must often balance different objectives (Michael et al., 2006), one of which is occupational safety. The (relative) importance of these objectives may (implicitly) be communicated to supervisors through job evaluation points (Zohar, 2000); as such, they may negatively affect occupational safety (Paté-Cornell, 1990). Another important indicator may be the load of occupational safety in terms of time. There are no clear guidelines or benchmarks on how much time supervisors should spend on safety. However, with safety professionals spending 'only' 59\% of their time on safety (Institute of Medicine [US] Committee to Assess Training Needs for Occupational Safety and Health Personnel in the United States, 2000), it is to be expected that 'regular' supervisors will spend less time on this issue. To gain an understanding of the extent to which occupational safety is present and manifest in supervisors' work, the following sub-research question was formulated:

What are supervisors' perceptions regarding the role of occupational safety in their job?

Underlying this question are considerations of job evaluation points, time spent on occupational safety, and the (changing) role of occupational safety in the work context.

\subsubsection{Supervisors' safety beliefs}

In addition to the perceived role of occupational safety in the work context, supervisors' beliefs regarding occupational safety may influence their work and the way they approach or handle occupational safety.

\section{Organisational safety motivation}

Related to the perceived importance of occupational safety that is (implicitly) communicated to supervisors, are beliefs about the authenticity of the motivation for safety within the organisation. As previous studies have demonstrated that organisations implement safety management systems to promote their image (Podgórski, 2000) or strategically report organisational safety performance (O'Neill, Flanagan, \& Clarke, 2016), it is interesting to examine the extent to which supervisors believe the motivation for occupational safety to be intrinsic and authentic within the organisation. 


\section{Responsibility for safety}

Supervisors may also differ in their beliefs regarding to the extent they are or should be responsible for occupational safety (Eakin, 1992), especially as views regarding responsibility have shifted from employer responsibility to a shared responsibility of the employer and the employee (Gray, 2006). Responsibility may influence safety behaviour as feelings of responsibility have been found to be predictive of behaviour in the contexts of ecology (Kaiser, Ranney, Hartig, \& Bowler, 1999) and academic achievement (Zimmerman \& Kitsantas, 2005). Lacking a clear and shared understanding of who is responsible may cause a diffusion of responsibility. This may have adverse effects, as seen in the bystander effect (Fischer et al., 2011).

\section{Beliefs regarding incidents and accidents}

Supervisors' beliefs regarding the preventability of accidents, factors contributing to incidents and accidents, and incident reporting are also of importance. Supervisor beliefs regarding the preventability of accidents in general can differ (LaCroix \& DeJoy, 1989; Zwetsloot, Aaltonen, Wybo, Saari, Kines, \& Op De Beeck, 2013), and may influence their willingness and their sense of a need for improved efforts (Rundmo \& Hale, 2003).

The same holds for incident reporting. To prevent future accidents from occurring, it is necessary to learn from past events and improve upon them (Drupsteen, Groeneweg, \& Zwetsloot, 2013). An organisation's ability to do so largely depends on the reporting of such events by employees, especially when it involves small incidents or near misses (Evans, Berry, Smith, Esterman, Selim, O'Shaughnessy, \& DeWit, 2006). Supervisors' influence on incident reporting has been demonstrated in previous studies (e.g., Clarke, 1998; Probst \& Estrada, 2010; Van der Schaaf \& Kanse, 2004). Therefore, both the extent to which supervisors believe incidents are already being reported and their beliefs regarding barriers that exist within the organisation are of importance. Perceived levels of incident reporting may be cause for extra efforts on behalf of the organisation, whereas the proper identification and subsequent elimination of barriers to reporting can increase the organisation's insights into problems that exist in the workplace.

Lastly, human action can occur at varying levels of consciousness (Döös, Backström, \& Sundström-Frisk, 2004). This also applies to behaviours related 
to safety. Indeed, previous research has found safety consciousness and awareness to be important for occupational safety (e.g., Sneddon, Mearns, \& Flin, 2013; Westaby \& Lee, 2003) and illustrates the influence of leaders on safety consciousness (Kelloway et al., 2006). Supervisors' beliefs regarding the consciousness with which employees enact their behaviours may direct their course of action. For example, the belief that incidents occur due to an employee not being consciously engaged in his or her work demands an increase in the employee's consciousness, whereas the belief that accidents are the result of conscious working employees may require different measures and actions.

To explore these beliefs among supervisors, the following sub-research question was formulated:

What are supervisors' beliefs regarding the motivation and responsibility for occupational safety as well as the preventability of, factors contributing to, and reporting of accidents?

\subsubsection{Supervisors' attitudes towards safety management instruments}

Because managers are responsible for delegating job tasks and managing the performance of their subordinates (Michael et al., 2006), to a certain extent, their subordinates' safety performance can be considered part of this responsibility. In monitoring and improving the safety of their subordinates, supervisors have certain instruments at their disposal that are often dictated by the organisation's policies. In their systematic literature review of 176 studies, Cornelissen, Van Hoof and De Jong (2017) list eleven instruments used to manage occupational safety within organisations: inspections, accident analysis and record keeping, safety representation, sanctions, rewards, accident reducing measures, training, (safety) communication, safety policies and procedures, and safety meetings and activities. Although Cornelissen et al. (2017) summarise and present results on the effectiveness of these safety management instruments, the attitudes of supervisors towards these instruments are not addressed. However, such attitudes may play an important role in managerial behavioural intentions and actual behaviour (Rundmo \& Hale, 2003), and supervisors may not feel comfortable propagating or imposing certain management policies (Eakin, 1992). 
DeHart-Davis, Chen and Little (2013) state that organisational rules differ from unwritten rules as the latter are 'disseminated verbally and stored in memory' (p. 333). However, others (e.g., Hale \& Borys, 2013; Reynaud, 2005) equate unwritten rules to routines, which DeHart-Davis et al. (2013) call norms or 'standards of appropriate behaviour' (p. 333). Ledeneva (2001) defines unwritten rules as the prescription of the ways in which both formal (organisational) and informal (social) constraints can be circumvented, their enforcement can be manipulated to one's advantage, and penalties can be avoided by creatively combining the elements of the rules of the game ( $\mathrm{p}$. 6-7). In light of occupational safety and all of the regulations that accompany it, unwritten rules are an interesting topic as they may both complement and interfere with occupational safety. They are therefore included in the list of safety management instruments.

A qualitative view can shed light on the willingness of supervisors to use these safety management instruments as well as their perceived effectiveness. Therefore, the following sub-research question was formulated:

What are supervisors' attitudes towards inspections, accident registration, accident analysis, sanctions, rewards, training, communication, meetings, (unwritten) rules and regulations, and safety-related activities?

\subsection{Method}

Supervisors from a major railway construction and maintenance organisation participated in semi-structured in-depth interviews that aimed to identify their views, beliefs and attitudes regarding (the management of) occupational safety.

\subsubsection{Procedure}

The interview started with a general explanation about the research, including the content of the informed consent form, topics that would be addressed, and the (expected) amount of time the interview would take. All interviews were recorded after permission was obtained. After the participants signed the informed consent form, demographic data were recorded. Then, the 
interview began with a discussion of the day-to-day activities and safetyrelated aspects of the interviewee's job. When the final topics were discussed, the interviewees were asked if there were any remaining topics that were not discussed but should have been. When this was the case, these topics were discussed before the interview was ended. Lastly, the interviewer thanked the interviewees for their time and answers.

\subsubsection{Instrument}

Semi-structured interviews were used to explore three main themes related to supervisors' perceptions of safety-related aspects. The first theme was related to the perceived role of safety in supervisors' day-to-day work. The discussion involved the participants' work evaluation points, the presence of occupational safety, and time spent on safety. The second theme was related to supervisors' safety beliefs. The discussion involved the participants' perceptions regarding safety motivation, the responsibility for occupational safety within the organisation, the preventability of accidents, incident reporting, and accident reasons. The third theme related to the participating supervisors' attitudes towards the management of safety and safety management instruments. Here, the interviewees were first asked what factors they thought facilitated and impeded safe work. Then, they were asked to reflect upon a list of widely used instruments to improve occupational safety. These instruments were partly extracted from a systematic review of 176 articles on occupational safety (Cornelissen et al., 2017). As the organisation has formal safety functions on various supervisory levels, safety representation as a factor was not explicitly included in the interview. We also chose to exclude accident-reducing measures as an explicit factor in the interviews as it is only loosely coupled with leadership. Lastly, the interviewees were again asked if there were any factors that could improve or impede occupational safety that had not yet been discussed; if this was the case, these were discussed.

\subsubsection{Sample}

Our sample consisted of 22 professionals involved in leadership functions (supervisors, managers, executives) within an organisation that is concerned with railroad construction and maintenance in the Netherlands. The participants were selected in a two-step procedure. In an earlier employee satisfaction survey conducted by the organisation employees' willingness 
to participate in safety-related follow-up research was inventoried. Second, from those employees willing to participate in follow-up research, a random selection was made that mirrored the managerial structure of the organisation. Potential interviewees $(N=30)$ were first approached via e-mail, resulting in a response rate of $73 \%$. All participating interviewees were male, with ages ranging from 40 to 60 years $(M=51.68, S D=5.32)$. The interviewees' tenure with the current company ranged from 3 to 37 years $(M=18.84, S D=9.07)$, and their overall tenure in this industry ranged from 3 to 38 years $(M=24.11, S D=9.41)$. The interviewees were grouped based on their supervisory position within the organisation: seven interviewees from higher management who were mainly involved in office work, eight foremen who functioned as a bridge between office employees and field employees, and seven group leaders who worked alongside the mechanics.

\subsubsection{Data analysis}

The 22 interviews yielded a total of 1362 minutes of recordings and were transcribed verbatim. The data reported in this manuscript were collected as part of a larger data collection. In the current manuscript, the focus is on all data concerning supervisors' attitudes and beliefs regarding occupational safety. The current study covers 260 pages of the total 314 pages of transcript.

Using ATLAS.ti software, the interview transcripts were analysed in three consecutive steps. First, the first author read the interviews and marked units of analysis that consisted of single or multiple sentences. Second, the units of analysis of five interviews (20\%) were coded by both the first author and a second, independent, lay coder, resulting in an overall Cohen's Kappa of .80. A Cohen's Kappa was also calculated for the three sub-research questions, resulting in Cohen's Ks of 82 (role of occupational safety in work), .85 (supervisors' safety beliefs), and .75 (supervisors' attitudes towards safety management instruments). Although the Cohen's Kappa of 'supervisors' attitudes towards safety management instruments' was satisfactory, the outcomes were discussed in a qualitative round as the Kappa did not meet the same standard as the other research questions. Third, the remainder of the interviews were coded by the first author. The coded units of analysis were then summarised and analysed per managerial level. 


\subsection{Results}

The results are presented with a focus on the perceived role of safety in supervisors' work, supervisors' safety beliefs, and supervisors' attitudes towards various safety management instruments. Each theme will end with a brief summary of the main findings.

\subsubsection{The role of occupational safety in supervisors' work context}

To assess the role of occupational safety in their job, the interviewees were first asked to list topics they thought to be part of the (annual) evaluation of them and their work. On average, four evaluation topics were listed, with foremen listing five on average and group leaders listing only half that number. Overall, safety was the most mentioned evaluation point, although it was not the most mentioned point in any of the separate managerial-level groups (see Table 3.1).

Table 3.1

Overview per managerial level of evaluation points mentioned more than two times.

\begin{tabular}{ccc}
\hline $\begin{array}{c}\text { Higher-level managers } \\
(n=7)\end{array}$ & $\begin{array}{c}\text { Foremen } \\
(n=8)\end{array}$ & $\begin{array}{c}\text { Group leaders } \\
(n=7)\end{array}$ \\
\hline Finances (6) & Inspections (6) & Quality (3) \\
Safety (4) & Toolboxes (6) & Safety (2) \\
Absenteeism (3) & Safety (3) & Leadership (2) \\
Incidents and accidents (3) & Leadership (3) & Instructions (2) \\
& Planning (3) & Solving breakdowns (2) \\
& Reporting (3) & \\
& Production (3) & \\
& Behaviour (3) & \\
\hline
\end{tabular}

Note: The number of times an evaluation point was mentioned is depicted between brackets. 
When asked which of the aforementioned evaluation topics was thought to be most important to their managers, of the 17 topics, seven of the 22 interviewees mentioned safety. In comparison, other evaluation points were mentioned twice at most.

The interviewees' estimates of the workload they spent on safety ranged from $5 \%$ to $50 \%$, with an average of $28 \%$. They spent this time on safetyrelated activities such as providing instructions, attending (toolbox) meetings, inspections, and the preparation of (safe) work. The time spent on safetyrelated issues differed a great deal among the managerial levels. The foremen and group leaders estimated their time spent as more than 20 percentage points higher than the higher-level managers. Although safety required a substantial amount of their work time, the majority of the interviewees were either content with this or wanted to spend more time on safety. Only three interviewees - all of them group leaders - wanted to spend less time on safety as they believed safety played an overly large role in their work. In line with the perceived importance of safety as an evaluation point in their work and the time spent on safety, almost all interviewees believed safety to play a substantial and continuous role in their job:

[Safety plays] a very important role. In the first instance, if I have doubts about the safety of my mechanics the work will not continue. And I will certainly do everything in advance to ensure their safety' M5, Foreman, 52 years old.

Additionally, the supervisors stated that safety should be understood more broadly as it was not limited to the direct workplace. The supervisors referred to the influence of employees' daily commute, especially after cold or rainy nights:

'It is not just purely the safety here [at work]. It is also some safety from and to work.' - M10, Foreman, 54 years old.

In addition to the influence of employees' daily commute, the supervisors referred to the impact that employees' private lives could have on safety at work: 
'Take a crane operator; for example; he could have a bad day. He could have an argument at home. You see him in the beginning when you give him instructions, and then he gets on his crane. And from there onward, he is just working. Well, then you cannot see if such a person did indeed have trouble at home.' - M28, Group leader, 48 years old.

When asked to look back, the interviewees agreed that the role of safety has changed over the last years, stating that it has become increasingly important and attracts more attention than in the past. This might be due to what Gray (2009) called the 'responsibilization of health and safety': a change characterised by a shift in responsibility from employers to employees, including supervisors. According to the interviewees, this increase is especially noticeable in the organisation and execution of work and in the fact that safety seems to have superseded production as the main focus in the work over the past years:

'Here in [location], we have many people who used to work on the tracks. In addition, I recently talked with a manager, and he stated, "I do not understand why it will not work because I used to do it this and this way". Then I try to convince him that the times have changed, that it is different now.' - M26, Group leader, 56 years old.

Despite the important role played by occupational safety in supervisors' work, one issue stands out: the occupational safety objective must compete with other objectives that supervisors have on their plate. This finding is line in with previous findings (Michael et al., 2006; Paté-Cornell, 1990; Rundmo \& Hale, 2003; Zohar, 2000). This competition is not only reflected in the number of evaluation points; a supervisor described this as follows:

'At first I was in a bit of a safety dilemma. For me, it felt like it was about creating more [safety] rules. And more rules mean less production, and I did not want that.' - M3, Higher-level manager, 51 years old.

In sum, the supervisors perceived occupational safety to play a substantial and increasingly important role in their job. For them, safety even played a role beyond the sphere of the workplace. However, despite the large and important 
role of occupational safety, supervisors indicated they must balance the safety objective with other organisational objectives, such as production, which can lead to time and capacity pressure.

\subsubsection{Supervisors' safety beliefs}

Although management commitment is an element that is widespread in the occupational safety literature, supervisors' safety beliefs and convictions are not well studied. Therefore, the topics of perceived safety motivation, responsibility, accident prevention, causes of accidents, incident reporting, and a perceived division in the organisation were addressed during the interviews.

\section{Organisational safety motivation}

The majority of the interviewees believed their management to be intrinsically motivated and the motivation for occupational safety within their organisation to be sincere, although four interviewees acknowledged that this was a change that had only occurred over the last couple of years and was even ongoing at the moment:

'We are really committed to people working safe. And we put in a lot of time, energy and money, but safety really is the motive.' - M13, Higher-level manager, 51 years old.

There were also interviewees ( $n=5)$-especially the foremen and group leaders- who thought the motivation to work safely was more extrinsically motivated, mainly because the client wanted it:

'[Client's name] prescribes it, so [our organisation] works accordingly. That's really simple. But I think that if it was up to [our organisation], then we would do it differently.' - M31, Group leader, 54 years old.

In line with this notion, among both the interviewees who believed the motivation for occupational safety to be intrinsic and those who believed the motivation to be extrinsic, there were interviewees who differentiated between working safely due to an intrinsic motivation and working according to the rules: 
'I think that the management says: "it is about the safe working philosophy." And I think that a very large part of the mechanics says that it is about the rules, complying with the rules, and the demonstrable compliance with those rules. And I notice that too.' M3, Higher-level manager, 51 years old.

\section{Responsibility}

Regarding the responsibility for occupational safety within the organisation, almost all interviewees stated that every individual has a share in the responsibility for occupational safety:

'The responsibility for safety lies in all layers of the organisation. Either way, the mechanic is responsible for his own safety. But uh, yes, I am also responsible for their safety. And finally, the VGCU is also responsible for safety, so the responsibility for safety is layered.' - M5, Foreman, 52 years old.

Although most interviewees agreed that the responsibility for occupational safety lies with everyone, 11 interviewees distinguished between an overall responsibility and a final responsibility:

'In the end, I am responsible for all of those men. And I and the managers that operate under me, we must ensure that those men are aware that the responsibility is with them, that they may act on that.' - M16, Higher-level manager, 59 years old.

In line with previous research (e.g., Eaking, 1992; Gray, 2006), a clear consensus on who was or should be responsible for occupational safety was lacking. Overall, the higher-level managers identified the responsibility to be with the organisation as a whole, higher-level management, or the VGCU (Safety and Health Coordinator during the Execution Phase). Whereas half of the foremen agreed that the final responsibility for safety lies with someone higher in the organisation, the other half of the foremen focused solely on the responsibility of the individual or group responsibility. At the group leader level, half of the group leaders mentioned that everyone is (proportionally) responsible for safety at work, and the others believed that those with specific safety functions on different levels should hold the final responsibility. 


\section{Preventability of accidents}

Although the strength of the interviewees' convictions differed from 'almost all accidents can be prevented' to 'I do not have any illusion that accidents and incidents can be reduced to zero', none of the interviewees believed incidents and accidents to be completely preventable. This conflicts with previous findings of Rundmo and Hale (2003). Despite this rather fatalistic view, the interviewees did mention that one should always strive to minimise the occurrence and impact of incidents and accidents. According to them, this could be best achieved by creating more barriers, taking the right precautions, proper preparation of work, being aware of the risks in one's work, and following procedures. Although the interviewees mentioned some solutions that could help to minimise the occurrence and impact of incidents and accidents, it is debatable whether supervisors would be willing to comply with those implementations, especially as some of the interviewees expressed that their beliefs contradicted the beliefs of the safety organisation:

'And you can never prevent everything, that's impossible.[...]. I think that the people who handle the reports, usually from the department of Quality, Working Conditions and Environment, always have an answer prepared: "You had to do that and that".' - M28, Group leader, 48 years old.

\section{Accident reasons}

Continuing along the line of incident and accident preventability, the interviewees were asked whether they thought accidents occurred due to conscious or more unconscious actions. Although not a single interviewee believed that anyone would deliberately cause an accident, two interviewees mentioned that accidents were sometimes caused by deliberate actions for which employees did not foresee the possible consequences:

'Well [long pause], those actions are conscious, but I think they are still unaware of the risks and dangers of those actions.' - M1, Higher-level manager, 57 years old.

Overall, and in line with previous research (O'Dea \& Flin, 2001), accidents were believed to be caused by unconscious actions. Furthermore, the interviewees mentioned several other factors that contributed to accident 
occurrence. The first factor is related to time. The interviewees mentioned time pressure and haste as the main factors contributing to accidents:

'I think acting too quickly, sometimes due to time pressure.' - M31, Group leader, 54 years old.

A second factor that was mentioned by multiple interviewees was the danger associated with a lack of focus:

'It's difficult to keep people alert. That's challenging; how do you stay focused, and how do you make sure your men stay aware?' M16, Higher-level manager, 59 years old.

A third factor mentioned by the interviewees relates to old habits. According to them, some employees find it difficult to accept that, with time, the (safety) rules have changed. This is especially the case for employees who had been accustomed to a certain way of working in the past that did not feel unsafe to them:

'A lot of our employees come from a different world, the rail world, but that was the rail world of 20,30 years ago. And back then the world was very different. Back then those rules did not exist, and you were allowed a lot more. Now we have rules that prevent you to act the same way you used to, and a lot of people find it difficult to accept that.' - M3, Higher-level manager, 51 years old.

Lastly, the interviewees addressed a variety of other factors that could negatively affect occupational safety, including encountering situations for which they were not prepared, solving errors in work planning, pressure experienced by those responsible for the work preparation, having one person responsible for too many incompatible functions, not applying a last-minute risk assessment (LMRA), the private use of cellular phones, and choosing convenience over safety.

\section{Incident reporting}

The majority of supervisors $(n=17)$ did not think that subordinates reported all encountered incidents, especially when those incidents were perceived as 
minor. These findings are in line with previous research (e.g., O’Dea \& Flin, 2001; Probst \& Estrada, 2010). It is noteworthy that, unlike the interviewees from the other managerial groups, the foremen were predominantly positive about the incident reporting behaviours of their subordinates:

'I think that employees were afraid to report incidents in the past [...] but now I feel like they are picking up on it and perceive it as something that is part of their work.' - M5, Foreman, 52 years old.

Whereas the foremen stated that the non-reporting of incidents was due to the unjustified perception of a situation as safe, group leaders perceived the effort needed to report an incident or accident to be the largest problem. Other reasons that were mentioned for not reporting incidents included the feeling that reporting was like a form of betrayal and the fear of negative consequences. Overall, these findings are in line with previous findings on the reasons for under-reporting among employees (Probst \& Estrada, 2010).

Supervisors on all levels agreed that increasing the visibility and follow-ups on reports and providing more insights into the purpose and importance of reporting were the most important ways through which incident reporting by subordinates could be improved:

'One of the things they always say is that nothing will be done with the reports. They say that for years. [...] and that they see why they have to report and what good comes from that.' - M1, Higher-level manager, 57 years old.

\section{Divided organisation}

During the interviews, a perceived gap between office employees and field employees emerged. Interviewees from all managerial levels made remarks related to this perceived gap, although those remarks were more present among the foremen and especially the group leaders. The higher-level managers perceived the gap as problematic as they were dependent on feedback from the people working outside: 
'We can think up anything we want here, but they are outside every day and they know what the reality is like. So they should provide feedback on it and tell us when it is nice that we came up with something, but that the situation outside is a bit different from our room next to the coffee maker.' - M16, Higher-level manager, 59 years old.

The foremen and group leaders focused more on the negative effects resulting from the gap as they experienced unworkable situations due to the misperception of office workers:

'A couple of years ago we had to do some inspections and measurements at night. The temperature of the rails was minus 29 [degrees Celsius]; that's how cold it was, and when it gets cold the rails shrink. So we do all our measurements and enter everything, and three weeks later, they ask us to do the same measurements again because "the numbers are too odd"' - M25, Group leader, 55 years old.

In addition, although the majority of interviewees believed that the gap could be reduced or diminished through exchanges between office employees and field employees, others were sceptical of this.

In sum, although occupational safety was perceived to be an important aspect of supervisors' work, the results are not as clear as one might expect for such an important topic. Not only did some interviewees doubt the intrinsic motivation for occupational safety within the organisation, but an explicit distinction was also made between working according to the rules and actually working safely. Additionally, it goes without saying that the apparent lack of clarity regarding who is or should be responsible for safety within the organisation is not beneficial to safety performance. Overall, the interviewees seemed to have a rather fatalistic and negative view of safety incidents and accidents. None of the interviewees thought that all accidents could be prevented, and the majority of interviewees thought that accidents were caused by unconscious behaviours. Other factors contributing to accidents mentioned by the supervisors included time pressure, a lack of focus, and old habits. Additionally, although the supervisors came up with ways in which 
incident reporting behaviours could be improved, the majority of interviewees stated that not all incidents were reported in line with corporate regulations. Lastly, a perceived gap between office employees and field employees emerged from the interviews. Although both groups had their own reasons for perceiving this gap as problematic, the majority of interviewees believed that the gap could be diminished through job rotation between office and field employees.

\subsubsection{Supervisors' attitudes towards safety management instruments}

The interviewees were asked to reflect upon the available safety management instruments. Due to the substantive overlap, the instruments were grouped into six categories: (1) inspections, accident registration and analyses, (2) sanctions and rewards, (3) training, (4) communication and meetings, (5) (unwritten) rules and regulations, and (6) safety-related activities.

\section{Inspections, accident registration and accident analyses}

The majority of interviewees had a positive attitude regarding the use of inspections, accident registration and accident analyses as means to improve occupational safety. However, they stated that their effectiveness mostly came from providing the proper feedback to employees:

'Analysing accidents and discussing them, yes that is effective. But in the end, feedback is the most important thing.' - M3, Higher-level manager, 51 years old.

Concerning the quantity of feedback, equal numbers of interviewees stated they were satisfied with the amount of feedback or that they wanted to receive more feedback on accidents that occurred.

The interviewees stated that conducting inspections was difficult and that inspections should not be used to 'catch' perpetrators but as a mean to increase safety awareness and find aspects that could be improved upon. Additionally, they expressed the concern that when employees are subjected to inspections, they show exemplary safety behaviours that deviate from their normal working behaviours. They believed that by conducting inspections 
unannounced or through external inspectors, the validity and effectiveness of inspections could be improved. Concerning the amount of inspections, opinions differed a great deal. Several interviewees advocated an increase in the amount of inspections, whereas others stated that there were already enough inspections. In contrast to inspections, most of the interviewees did not deal directly with accident registration and analyses. However, there seemed to be considerable ambiguity concerning the registration of incidents and accidents as different interviewees mentioned different ways through which these should be registered. The interviewees mentioned that they registered incidents and accidents through the error coordinator, their supervisor, and the online platform.

\section{Sanctions and rewards}

The interviewees were divided on the use of sanctions as a mean to improve occupational safety. Although several interviewees mentioned that they did not know the specifics of the organisation's sanction policy, a slight majority of the interviewees believed sanctions to be effective in addressing safety. However, the interviewees from different managerial levels -especially the foremen - were reluctant to use sanctions:

'Sanctions, yes, I think they help [to improve safety], but I do not think it's a good form. It's a form that puts down negative pressure.' - M3, Higher-level manager, 51 years old.

However, they thought the use of sanctions to be justified if someone repeatedly made mistakes:

'When someone makes the same error three times within a certain period of time, then you can move on to sanctions' - M10, Foreman, 54 years old.

In contrast to the varying attitudes towards sanctions, the majority of interviewees thought rewards were an effective way to improve occupational safety. The interviewees also held a broad view regarding rewards. In particular, the higher-level managers and foremen indicated that rewards could and/or should come in a variety of formats and not only include financial rewards: 
'Rewards are fine. Can be a pat on the back, can be a bouquet of flowers, can be a gift voucher.' - M12, Higher-level manager, 51 years old.

However, there were also concerns regarding the use of rewards to improve occupational safety, especially among foremen:

'Look, you're rewarded every four weeks when you receive your salary. That's your salary, you get paid for the work you do. [...] Why should you need to reward someone for doing his work?' - M8, Foreman, 57 years old.

The interviewees - mostly group leaders - also expressed concerns that awards were only given to senior-level managers or were awarded in unfair ways, and supervisors throughout the managerial levels indicated that rewards should be awarded more often:

II think we should reward more often, and that would also help in improving [safety].' - M1, Higher-level manager, 57 years old.

\section{Training}

The interviewees mentioned a broad spectrum of different training formats, including the different internal and external training institutions and a specially developed trivia game. Supervisors from all managerial levels were positive towards the training within the organisation. They believed there was plenty of time and budget available for training. Some of the interviewees even felt that they and their employees received too much training. This was especially the case for training they did not perceive as necessary to perform their own work:

'I think we receive more than enough training. [...] A lot of things are being taught actually, that actually do not even matter for us.' M25, Group leader, 55 years old. 


\section{Communication and meetings}

On all levels, the supervisors stated that communication was an important element in (improving) occupational safety, and some of the interviewees stated it was also an effective means to improve occupational safety:

'Communication is key; it plays a role in everything. [...]

Communication is one of the most important things.' - M29, Group leader, 40 years old.

As for communication in general, the general attitude of the interviewees regarding meetings was positive. They believed meetings to be effective and important, and, overall, they were satisfied:

'I'll stick to it; for me, meetings are the best way to improve safety, especially when those meetings are interactive.' - M1, Higher-level manager, 57 years old.

Interactivity was underscored by multiple interviewees as an important element in safety communication and meetings in particular. The interviewees agreed that there were not too many meetings; they stated that there were many, enough or not enough meetings.

Although the majority of the interviewees believed that communication and meetings were important, some of the interviewees commented that communication itself and the planning of meetings was not something that came easily. Regarding communication, eight interviewees -of whom six were group leaders - underscored that proper trainings are crucial as there is still room and need for improvement:

'Communication, in all respects, yes, I think it can always be improved. Communication is not something that comes natural to us humans. We are always missing the mark with that.'- M5, Foreman, 52 years old.

Concerning the planning of meetings, the foremen and group leaders stated that it was difficult to plan meetings that could be attended by everyone: 
'I would like to meet the guys more often to discuss things. We are always one step behind on the real situation outside, but we are ruled by our planning' - M18, Foreman, 45 years old.

\section{(Unwritten) Rules and regulations}

Although the interviewees agreed upon the necessity of rules and regulations, the vast majority believed that, at the moment, there were enough or even too many rules and regulations:

'Rules and regulations are necessary; otherwise we will mess up. [...] in our current work situation there are enough rules and regulations. [...] at a certain point we do not see the wood for the trees anymore.' - M16, Higher-level manager, 59 years old.

The majority of interviewees believed unwritten rules existed in their field, whereas a quarter of the interviewees stated they did not have any insight into the existence of unwritten rules.

Concerning the effectiveness of rules and regulations, the interviewees warned that an excessive amount of rules and regulations would endanger employees' critical thinking process:

'We as an organisation should not try to create a rule for every situation to explain what you are and are not allowed to do. People should be able to keep thinking for themselves because otherwise you will smother them. [...] They must be able to keep thinking for themselves.' - M18, Foreman, 45 years old.

Further, the foremen and group leaders stated that too many rules and regulations could cause employees to stop reading the rules and regulations:

'You are expected to read all of them [rules and regulations]. But at a certain point you will stop reading them. Then it dilutes.' - M26, Group leader, 56 years old.

Surprisingly, there were more interviewees who believed that unwritten rules made a positive contribution to occupational safety than those who stated that 
unwritten rules had a negative effect on safety. One interviewee warned of the possible consequences of unwritten rules:

'The difficulty with unwritten rules lies in the unambiguity of the agreement; what do you precisely agree on? And if someone else interprets that unwritten rule in a different way, what are the consequences? And if that unwritten rule moves from one area to another area, is it still understood the same way? So yes, I think it to be a big risk.' - M3, Higher-level manager, 51 years old.

\section{Safety-related activities}

In discussing safety-related activities, the majority of interviewees referred to the training and education days that occurred during the winter. Two other elements that were mentioned more than once were a teambuilding day and a special safety day. In general, the interviewees were positive about the training and education days as they perceived them as useful, especially after the improvements that were implemented over the last few years.

In sum, the results demonstrate that supervisors differ in their attitudes towards both the effectiveness and optimal quantity of safety management instruments, and they have reservations with regard to the application of such instruments. Although the supervisors were predominantly positive regarding inspections, accident registration, and accident analyses, they thought their effectiveness could be improved. Although the supervisors were divided on the use of sanctions, they were more positive regarding the use of rewards. Furthermore, although the supervisors acknowledged communication to be difficult, it was seen as both important and effective in addressing occupational safety issues. The importance of communication was also stressed for inspections, accident registration, and accident analyses. Lastly, the supervisors thought there were enough or even too many rules, and they were surprisingly positive about unwritten rules. 


\subsection{Discussion}

Considering the important role of supervisors in maintaining and improving safety in the workplace, this study aimed to increase current knowledge on supervisors' perceptions and beliefs regarding occupational safety and supervisors' evaluations of safety management instruments.

\subsubsection{Theoretical contributions and future research}

This study makes several contributions to existing theory that give rise to further research. First, some of the supervisors stated that factors other than intrinsic motivation played a role in the organisational motivation for occupational safety. In line with earlier studies (e.g., O'Neill et al., 2016; Podgórski, 2000), this indicates that organisations may have alternative motives for pursuing occupational safety. We recommend that future researchers further explore the occurrences of this possible 'safe-washing' phenomenon and investigate whether such perceptions matter for improving occupational safety outcomes.

Second, concerning the responsibility for occupational safety, the supervisors distinguished between individual responsibility and a final responsibility attributed to those higher in the organisation. This dichotomy may be the result of the shift in responsibility that Gray $(2006 ; 2009)$ describes and provides cause for concern as feelings of responsibility have been found to be predictive of behaviour in other contexts (e.g., Kaiser et al., 1999; Zimmerman \& Kitsantas, 2005). Additionally, the lack of a clear and shared understanding of who is responsible may have adverse effects (Fischer et al., 2011). In sectors that are characterised by high levels of segregation in time and space between supervisors and subordinates, such as the one studied here, supervisors face the challenge of fostering feelings of responsibility among their subordinates as they themselves are not present to hold the employees accountable (Geller, 2005). However, Gray (2009) warned that employees experience this individual responsibility as a heavier burden due to their direct exposure to hazards. Future research should explore how differences in the attribution of responsibility for safety affect supervisors' willingness and sense of need to improve occupational safety, (feelings of) occupational safety and safety performance. 
Third, in contrast to previous research (LaCroix \& DeJoy, 1989; Rundmo \& Hale, 2003; Zwetsloot et al., 2013), the supervisors were unanimous in that none of them believed that all accidents could be prevented. Additionally, a majority of the supervisors thought that accidents resulted from unconscious actions. Previous research found that managers' behavioural intentions and behaviour were related to safety attitudes (e.g., Rundmo \& Hale, 2003) and that managers' behaviour related to employee safety consciousness (e.g., Kelloway et al., 2006). Therefore, future research should investigate how and to what extent varying safety beliefs regarding accident preventability and accident reasons influence the willingness and sense of need of supervisors to increase safety efforts.

Fourth, given the importance of learning from and improving upon past events (e.g., Drupsteen et al., 2013; Evans et al., 2006), it is striking that all of the supervisors stated that some incidents were not reported. Although several barriers were mentioned by the supervisors in our study, they were not unique to this particular organisation and overlap with the barriers identified in earlier studies (e.g., Clarke, 1998; Evans et al., 2006; Van der Schaaf \& Kanse, 2004). Consequently, future research should focus not only on the identification of factors that stand in the way of incident reporting but also on finding ways to diminish or overcome such barriers within organisations.

Fifth, a majority of the supervisors believed that unwritten rules had a positive effect on occupational safety. Their positive attitudes may be explained by the way unwritten rules can be used to circumvent both formal and informal constraints (Ledeneva, 2001). However, in line with DeHart-Davis et al. (2013), one supervisor warned of the dangers of unwritten rules, stating that they do not easily allow for clear mutual understanding and that they are open to interpretation, creating a potential threat to occupational safety.

Lastly, in the responses regarding the various safety management instruments, the perceived importance of communication stands out. This finding is in line with previous research (Cornelissen et al., 2017). Although the supervisors perceive communication to be difficult, they view it as a necessary and effective means to improve occupational safety. Additionally, they consider communication to be part of (the success of) other safety management instruments, such as inspections, accident registration and accident analyses. According to the supervisors, the strength of these instruments lies in the 
feedback provided based on their outcomes. Clear and open communication could also help supervisors and employees come to a mutual understanding and prevent the misinterpretation of unwritten rules. Additionally, communication could help employees to better understand the importance of safety-related activities and increase the acceptance of such activity days. Given the importance of communication for occupational safety in general, its perceived importance for other safety-related factors, and supervisors' difficulty with communication, future research should focus on the safety communication concept and its underlying facets.

\subsubsection{Practical applications}

The findings illustrate the importance of taking supervisor beliefs and attitudes into account in the management of occupational safety. As the workload and the importance of safety have increased over the last years, organisations should re-assess the task loads of their supervisors. Additionally, organisations should be aware that they might communicate the (relative) importance of occupational safety to supervisors through job evaluation points (Paté-Cornell, 1990; Zohar, 2000).

The lack of clarity on who is or should be responsible within the organisation for occupational safety is problematic and requires organisations to explicate everyone's safety responsibilities. If it follows from this that every employee has a share in the responsibility for occupational safety, they should be trained to exercise their responsibility and the right to refuse unsafe work (see Gray, 2006). The finding that none of the supervisors believed that all accidents could be prevented may influence their willingness and sense of need to act upon the prevention of accidents (Rundmo \& Hale, 2003). Organisations should therefore look for ways to motivate supervisors to carry out their occupational safety task(s) as effectively as possible. One of the factors contributing to accidents mentioned by the supervisors was related to old habits. They stated that employees find it difficult to accept that (safety) rules have changed, especially as those employees were accustomed to a way of working that they did not experience as unsafe. Organisations should therefore look for ways to break down those old habits among tenured employees, such as by fostering a culture in which it is normal to provide coworkers with feedback (Laitinen \& Ruohomäki, 1996) or by means of training programmes for tenured staff. Related to this is the problem with incident 
reporting, especially for incidents perceived as minor. Organisations should find ways to emphasise and clarify the need for incident reporting and foster a safety climate in which this is not only accepted but also the norm. The gap between office and field employees that was perceived as problematic could be minimised or even diminished through job rotation, as suggested by the supervisors in the current study and previous research (e.g., Huang, 1999; Song, Xie, \& Dyer, 2000).

Lastly, supervisors' attitudes towards the various safety management instruments may also affect their willingness and sense of need to act upon the prevention of accidents (Rundmo \& Hale, 2003). Although in the current study the supervisors' overall attitudes were positive, the expressed unwillingness to use sanctions, despite viewing them as an effective means, the lack of knowledge of the organisation's sanction policy, and the aversion to use inspections as a mean to 'catch' perpetrators illustrate the importance of taking supervisors' attitudes into account in safety interventions and in the dissemination of safety policies within the workplace.

\subsubsection{Limitations}

Although we are convinced that the results presented here contribute to the current knowledge and understanding of supervisors and their role in occupational safety, there are some limitations that need to be addressed. First, the results were collected in a single organisation that operates in a specific sector, which affects the generalisability of the results. Second, as the organisation operates in a high-risk environment, considerable attention is paid to occupational safety. This, combined with the interviewees' knowledge that the interview concerned occupational safety, could have affected the answers provided. Third, although the three groups of supervisors were comparable in size, they were also limited in size. Consequently, the representativeness of these groups may be influenced. Lastly, the qualitative data do not allow for a quantitative comparison among the groups or for any statistical inferences among perceptions, beliefs and attitudes on the one hand and safety outcomes on the other hand. The latter provides a possible avenue for future quantitative research into the effects of supervisors' attitudes on organisational and employee safety performance. 


\subsection{Conclusion}

In light of the important role of supervisors for occupational safety and the importance of beliefs and attitudes for behaviour and behavioural intention, this study explored supervisors' safety perceptions, beliefs, and attitudes. The results illustrate that supervisors perceive safety as an increasingly important and extensive task that competes with other objectives. The results provide helpful insights into supervisors' safety beliefs, indicating that supervisors hold differing views regarding safety and accidents and approach occupational safety in different ways. Lastly, the results illustrate supervisors' attitudes and daily routines in the use of safety management instruments, inspections, accident registration, accident analysis, sanctions, rewards, training, communication, meetings, (unwritten) rules and regulations, and safetyrelated activities.

\section{Acknowledgements}

This research did not receive any specific grant from funding agencies in the public, commercial, or not-for-profit sectors. 


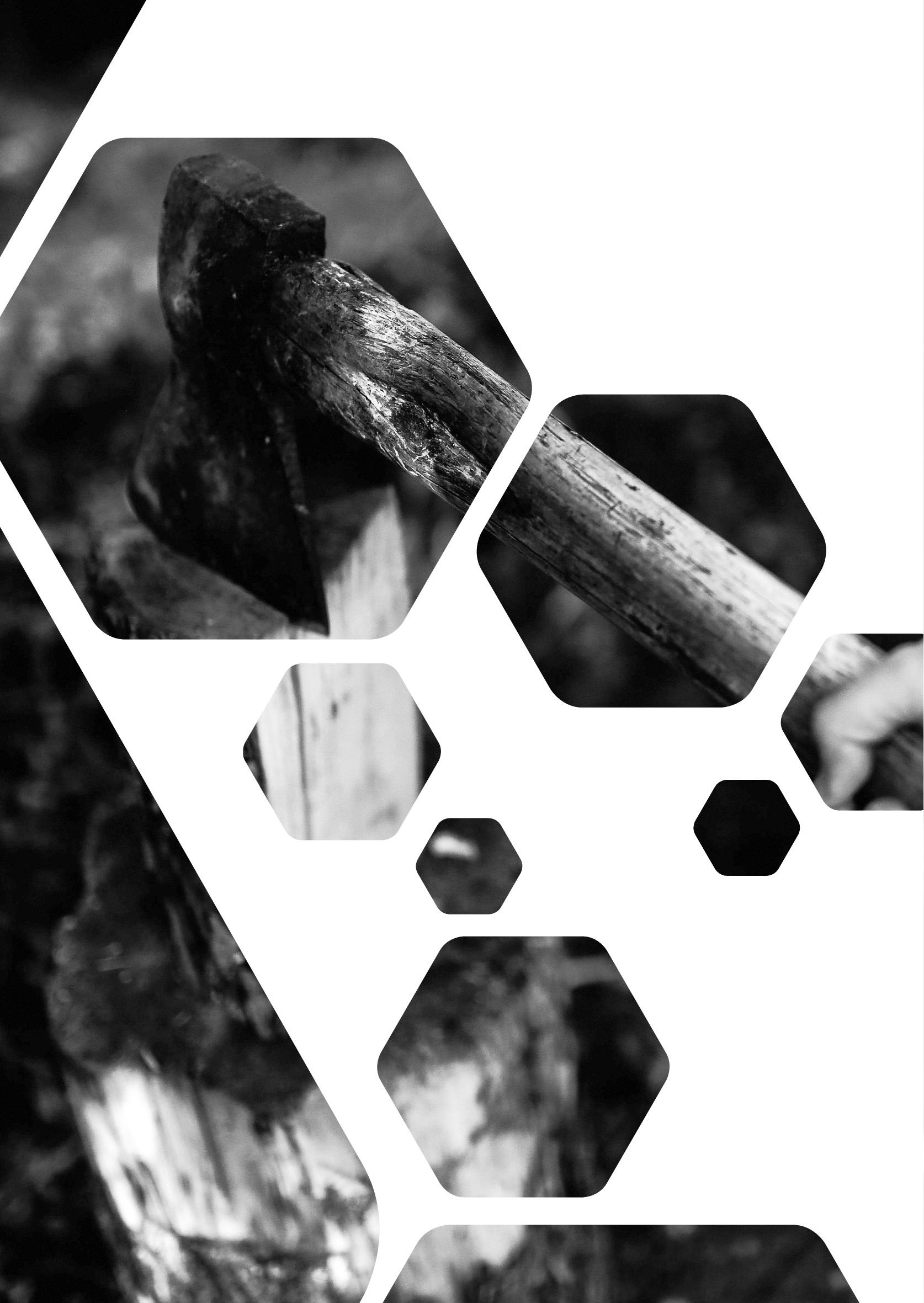


Chapter 4

\section{How logical is safety? An institutional logics perspective on occupational safety}

'When the woodcutter brings down his axe on the wood, it can be a case of wage labor, provision for one's household, or form of recreation.'

Richard Swedberg

Cornelissen, P.A., Van Vuuren, H.A., \& Van Hoof, J.J.

How logical is safety? An institutional logics perspective on occupational safety Under review 


\subsection{Introduction}

Occupational accidents are still commonplace in the contemporary workplace, despite increased understanding of occupational safety, human psychology, and human-technology interaction (e.g., Alarcón, Acuña, Diethelm, \& Pellicer, 2016; 'Safety and health at work', n.d.; Villanueva \& Garcia, 2011). Researchers and practitioners not only struggle to understand, reduce and prevent occupational incidents and accidents but also seemingly view incidents and accidents as a result of irrational behaviours and decisions (Hopkins, 1999). However, dismissing behaviour as irrational is 'simply an admission that the behaviour is inexplicable in terms of the concepts to hand' (Hopkins, 1999, p. 148). Dismissing behaviour as irrational also makes it difficult to develop policies and interventions to counter the behaviour. Using a new approach to occupational safety, our aim is to yield new insights leading to an improved understanding of what drives safety-related decisions and behaviours. In the present study, we propose the perspective of institutional logics (Friedland \& Alford, 1991; Greenwood, Raynard, Kodeih, Micelotta, \& Lounsburry, 2011; Thornton, Ocasio, \& Lounsbury, 2013) for a multisided understanding of occupational safety.

Consider the following situation:

Three construction workers (Peter, Marcel and George) see an employee from headquarters enter the construction site to deliver a parcel without wearing a helmet. All three workers have their own response. Peter remembers the client stressing the importance of finishing the work in time, and he decides not to interfere as, this might negatively affect the work pace. Marcel recognises the employee as a higher-level manager and decides not to interfere because he doesn't want to correct someone who outranks him. Being familiar with the worksite and the dangers associated with not wearing a helmet on site, George grabs a spare helmet and gives it to the visiting employee.

In this example, three employees make different decisions that are rooted in different manners of thinking about the situation: a task focus, hierarchy awareness, and practical solutions. Behaviours and decisions at a given moment are based on the knowledge, perspective and understanding people 
have of the situation at that time, something that is called 'local rationality' (Dekker, 2003). We will explore how viewing occupational safety issues through the lens of different institutional logics provides a pragmatic avenue for identifying such local rationalities and analysing the complexities associated with them, as there are distinct institutional logics present in organisational contexts that each provide their own coherent rationale for safety (Thornton \& Ocasio, 2008). A logic ultimately comes down to the root metaphors that people use to view reality (Friedland \& Alford, 1991). This creates a coherent logic as people define rationality from a certain perspective. The use of an institutional logics approach thus allows for the explanation of behaviour beyond the assumption that people think alike about the consequences of working unsafely. Depending on the specific dominant logic, people will differ in their assessment of what is (in)appropriate, (ir)rational, and (un)safe in the safety context.

The most widely accepted set of ideal types of institutional logics, which we therefore also apply in the present study, was proposed by Thornton et al. (2013). This set comprises seven institutional orders: the market, the profession, the corporation, the state, the family, the community, and the religion. Each of these orders has its own distinct logic that provide guidelines for the interpretation and functioning in social situations (Greenwood et al., 2011, p. 318). This set of logics has proved effective in studying how multiple logics impact government initiatives to improve primary health care in Denmark and Canada (Waldorff, Reay, \& Goodrick, 2013), how the family logic influenced the organisational form and practices of Italian law firms (Fairclough \& Micelotta, 2013), and the impact of (regional) state and family logics on organisational responses to a market logic (Greenwood, Díaz, Li, \& Lorente, 2010).

The present paper demonstrates how the discourse of logics unveils manners of thinking, speaking, and acting about safety within organisations. Applying such a discourse uncovers, illustrates, and explains the dynamics that are overlooked by traditional methods of studying occupational safety. We have three aims with this introduction of logics in the safety context. First, we aim to reveal the different logics at play when safety is discussed. The multiplicity of logics and their settlement in organisations enables us to better understand why occupational safety often fails. Second, we aim to explain how employees apply those different logics and thereby differ in terms of their approach 
to occupational safety, which can be traced by seeing how they explicitly articulate their logic. Third, an increased understanding of the various logics can contribute to finding solutions that minimise the amount of conflict within organisations, which depends on the manners in which the multiple logics are treated in a given situation. This paper is structured as follows: first, we will provide descriptions of the seven ideal institutional orders proposed by Thornton et al. (2013) and of logic multiplicity. Second, we will use this logics lens to analyse qualitative data gathered among managers in a railway construction and maintenance organisation and illustrate the presence of institutional logics and occurrences of logic multiplicity with regard to occupational safety. Third, we will discuss the theoretical and practical implications of these findings.

\subsection{Theoretical framework}

Institutional logics are 'the socially constructed, historical patterns of material practices, assumptions, values, beliefs, and rules by which individuals produce and reproduce their material subsistence, organize time and space, and provide meaning to their social reality' (Thornton \& Ocasio, 1999, p. 804). Greenwood et al. (2011) summarise the content of this pattern by describing how institutional logics 'provide guidelines on how to interpret and function in social situations' (p. 318). Such institutional logics define what is considered valuable within a cultural system (Friedland \& Alford, 1991, p. 232) and what is considered to be the most rational choice to make. Combined, these cultural systems, or institutional orders, 'compose the key cornerstone institutions of society' (Thornton et al., 2013, p. 53). Logics are the more subtle and abstract underlying mechanisms that guide institutional orders and social meaning (Johanson \& Waldorff, 2015). Thornton et al. (2013) distilled a generally accepted set of seven ideal types of institutional orders, treating the institution from the perspective of the market, the profession, the corporation, the state, the family, the community, and the religion. The institutional logic is the basic unit, and field logics, organisation logics, and individual logics emerge from there and are 'nested within these societal-level logics' (Besharov \& Smith, 2014, p. 366). We will summarise each of the orders (see Table 4.1).

Within the institutional order of the market, the focus is on 'the accumulation, codification, and pricing of human activity' (Thornton et al., 2013, p. 44). 
Central to the market logic is that efficiency underlies profitability, business practices are determined by the maximum value possible from transactions, and firm reputation is based on success in the market (Reay Jaskiewicz, \& Hinings, 2015). In the ideal type 'market logic', the organisation is organised with a focus on profitability, and its processes are developed to maximise efficiency. As such, it competes for consumer preferences and choice to increase sales and/or market share with other, similar organisations in a free and unregulated market (Goodrick \& Reay, 2011; Reay et al., 2015). The influence of the market logic is exemplified by Glynn and Lounsbury (2005) in their study of critics' reviews of a symphony orchestra.

In the order of the profession, individual members form organised bodies who 'create knowledge and belief systems that define arenas of interest and jurisdiction' (Thornton, 2002, p. 83). The basis for the autonomy and authority of professionals stems from the legitimacy of a mission and the professionals' 'exclusive ability to apply expert and esoteric knowledge to particular cases' (Thornton, 2002, p. 83). The organisation structure of the professional is based on individual relations. As such, it is often viewed as antithetical to the hierarchical means of control of corporations (Thornton, 2002). In the ideal type 'profession logic', 'professionals rely on abstract knowledge to conduct their practice solely or in partnership with others of the same profession, allowing for professional control over the content and organization of work.' (Goodrick \& Reay, 2011, p. 378). The logic of the profession has been exemplified by Jones, Livne-Tarandach and Balachandra (2010) in their study on written pitches by architecture firms.

The order of the corporation is centred around the organisational structure of the organisation with its hierarchical levels. Its sources of legitimacy stem from the market position of the firm, and authority is exercised by the board of directors and top management. In the ideal type 'corporate logic', work is encoded in routines of the firm and is subject to the administrative control of managers in a hierarchy (Thornton, 2004). Managers are in charge of determining the content and organisation of work, evaluating worker performance, and the appropriate price and quality of services and products (Goodrick \& Reay, 2011). The influence of the logic of the corporation was exemplified in a study by Thornton and Ocasio (1999) on the higher education publishing industry, where they found that rank in the organisational hierarchy was seen as one of the key sources of legitimacy in the industry 
and that capital was committed to the firm, instead of to individual editors (Thornton \& Ocasio, 1999).

The institutional order of the state is focussed on converting 'diverse issues into consensus or majority vote' to increase community good (Thornton et al., 2013, p. 44). Its source of legitimacy stems from democratic participation and authority is exercised through bureaucratic domination (Thornton et al., 2013). In the ideal type 'state logic', the government is responsible and in control, determining the appropriate quality standards and prices for services and products (Goodrick \& Reay, 2011, p. 379). The influence of the state logic has been exemplified by Pahnke, Katila and Eisenhardt (2015), who studied its influence on young firms and innovation.

The institutional order of the family comprises the family logic, in which the focus is on 'converting social relations into reciprocal and unconditional obligations oriented to the reproduction of family members' and 'beliefs are reinforced through rituals' (Thornton et al., 2013, p. 44). As Reay et al. (2015) explain, in the family logic, an 'unconditional loyalty to family underlies business practices', the firm's identity relies on the family reputation, and status within the firm is determined by family membership (p. 8). In the ideal type 'family logic', key decisions are made by family members and guided by family tradition, and the firm is organised to benefit family members (Reay et al., 2015). The family logic is also exemplified in Japanese corporate networks and Japanese management practices by Bhappu (2000).

From the institutional order of the community follows the community logic. Communities are created through common boundaries or territories that create common goals (Thornton et al., 2013; Reay et al., 2015). Actions are driven by emotional connections between participants, and cooperative actions should benefit all (Reay et al., 2015), not just the 'common economic needs of the communal economy' (Thornton et al., 2013, p. 68). In the ideal type 'community logic', competition between organisations in the same field is avoided, as they are organised to fit with each other and decisions are made that should benefit all, not just one organisation (Reay et al., 2015). The community logic is exemplified in research regarding activists' protests aiming to protect local business communities from chain stores (e.g., Ingram \& Rao, 2004; Ingram, Yue, \& Rao, 2010). 
The last and least studied institutional order is that of the religion (Tracey, 2012). Friedland and Alford (1991) initially described the institutional logic of the religion as being centred around truth, similar to science. However, Friedland (2001) later agrees with Becker's critique that this description should be rejected. Instead, Friedland (2001) states that 'the institutional logic of the religion centres on the order of creation, locating humanness in the cosmos, replicating cosmology through ritual, a practical metaphysics that necessarily points before life and after death' (p. 134). This notion is also more present in the definition that Thornton et al. (2013) provide, who state that the order of the religion focuses on 'an explanation of the origin of the world and in converting all issues into expression of absolute moral principles on the basis of faith' (p. 44). The logic of the religion is exemplified in Friedland's (2002) essay about religious nationalism.

\subsubsection{Multiplicity of logics}

If every stakeholder considers the same set of values, beliefs, rules and behaviours as rational, they adopt a single logic. Often, however, multiple logics are present within organisations (Besharov \& Smith, 2014; Greenwood et al., 2011). One reason for this multiplicity is that organisations that strive for stability and external support incorporate a variety of incompatible structural elements (Meyer \& Rowan, 1977, p. 356). Such multiplicity of logics will probably lead to confusion because the participants in the interaction do not share the same set of values, definition of rationality and reality. Moreover, adopting one order as the logical manner of thinking easily leads to considering an alternative order as morally questionable, irrational or delusional. A typical example of such a clash can be found in the healthcare field, in which the logics of the market and the profession collide. In their analysis of the medical field, Schwartz and Scharpe (2010) state that 'the commercialisation of medicine demoralises the practice of medicine and, in doing so, it threatens to "de-moralise" doctors' (p. 199).

Whereas early work about logic multiplicity framed the relationship between conflicting logics in terms of dominance (Nicolini, Delmestri, Goodrick, Reay, Lindberg, \& Adolfsson, 2016), as if one logic won, later research associated logic multiplicity with increased durability, sustainability, and innovativeness (Besharov \& Smith, 2014). Additionally, research found that multiple conflicting logics can coexist for long periods of time or remain unresolved 
(Nicolini et al., 2016), and organisations that could not achieve their goals with one singular logic were identified (Johansen \& Waldorff, 2015). In order to account for these conflicting outcomes of logic multiplicity research, Besharov and Smith (2014) developed a framework that allows for the categorisation of organisations along two (continues) axes: the degree of logic compatibility (i.e., the extent to which logics provide (in)compatible prescriptions for action) and the degree of logic centrality (i.e., the extent to which one or multiple logics are core to organisational functioning). On the outer edges of these axes, Besharov and Smith (2014) identify four ideal types of organisations incorporating multiple logics: (1) contested organisations, (2) estranged organisations, (3) aligned organisations, and (4) dominant organisations.

1. Contested organisations are characterised by high centrality and low compatibility, leading organisational members to hold 'competing expectations about appropriate organizational goals and lacking a clear guideline as to which goals should prevail' (p. 371).

2. Estranged organisations are characterised by low centrality and low compatibility. Here, conflicts arising from multiple logic are tempered, as leading internal conflicts can be 'resolved in favor of the dominant logic' (p. 372).

3. Aligned organisations are characterised by high centrality and high compatibility. Despite the presence of multiple logics, conflict is likely to be minimal, as the organisational goals implied by the different logics are consistent, and organisational decisions do not imply 'either/or choices' ( $p$. 373).

4. Dominant organisations are characterised by low centrality and high compatibility. The low likelihood of conflict arising from logic multiplicity results from the consistency of other logics with the primary logic and compatible prescriptions for actions from the different logics.

With their framework, Besharov and Smith (2014) focus on the implications of logic multiplicity for internal conflict, as it is a central concern in previous research about logic multiplicity. 


\subsubsection{Applying logics to occupational safety}

We propose that an institutional logics perspective provides new methods to uncover, illustrate, and explain the rationale(s) underlying safety-related thinking, decisions and behaviours and that awareness, acknowledgement, and understanding of these different rationales can further improve workplace safety and are much needed to do so.

Institutional logics 'provide guidelines on how to interpret and function in social situations' (Greenwood et al., 2011, p. 318), defining what is considered valuable within a cultural system (Friedland \& Alford, 1991) and what is viewed as the rational choice. As such, occupational safety should not be viewed as a distinct logic, although logics can be used to explore and explain occupational safety (cf. Daudigeos, Boutinot \& Jaumier, 2013; Madsen \& Hasle, 2017). As different logics presume different ideas about occupational safety, and uncovering those different ideas provide insights into what makes occupational safety so difficult to address, their identification and interpretation can help create safer workplaces. Viewing existing research through an institutional logics lens reveals that others have already implicitly illustrated the presence of institutional logics in the occupational safety context. For example, Swuste et al. (2010) provide examples of the state logic -'Various British commissions have reported on working hours, child and women's labour, leading to the start of social legislation in 1802' (p. 1001)and the religious logic - 'In this period, people generally have the view that disease and doom are part of the plan of the Almighty' (p. 1001). Hopkins (1999) illustrates the market logic: 'It was economically rational for such employers to spend money on a variety of preventive measures' (p. 146). Reader and O'Connor (2013) illustrate the presence of the corporate logic: 'Although the responsibility for decision making lay with the Captain, crew members were unclear as to who was in charge due to missing handover procedures' (p. 416). Implicit illustrations of the presence of opposing logics are also visible in existing safety literature: 'According to the liberal philosophy, these [safety] initiatives should not be initiated by government, but by private initiative' (Swuste et al., 2010, p. 1002). An overview of the defining characteristics for rationality and an application to occupational safety of these orders can be found in Table 4.1. To explore and illustrate the applicability of logics to the safety context, we analysed data from an organisation in which safety issues arose due to different logics. 
Table 4.1

An overview of key references, the defining characteristics of the seven interinstitutional orders, and their application to safety.

\begin{tabular}{|c|c|c|}
\hline Logic & $\begin{array}{c}\text { Defining characteristics for } \\
\text { rationality }\end{array}$ & $\begin{array}{c}\text { Application to occupational } \\
\text { safety }\end{array}$ \\
\hline $\begin{array}{l}\text { Market } \\
\text { order }\end{array}$ & $\begin{array}{l}\text { Priority is given to choice, } \\
\text { quality and profit. } \\
\text { Values are competition and } \\
\text { customer preference. } \\
\text { - Success is a larger share and } \\
\text { profit. }\end{array}$ & $\begin{array}{l}\text { Safety is considered an asset for } \\
\text { gaining market share. Although } \\
\text { safety might decrease efficiency, } \\
\text { securing it might outweigh } \\
\text { the costs associated with } \\
\text { incidents, accidents, and (lost } \\
\text { time) injuries, and can give the } \\
\text { organisation and advantage in } \\
\text { consumer choice. }\end{array}$ \\
\hline $\begin{array}{l}\text { Profession } \\
\text { order }\end{array}$ & $\begin{array}{l}\text { Priority is given to the opinion } \\
\text { of credible members of a } \\
\text { professional community. } \\
\text { - Values are membership, } \\
\text { expertise, training, and } \\
\text { mentoring. } \\
\text { - Success is a product of } \\
\text { high quality provided by } \\
\text { professionals, socialised within } \\
\text { their community. }\end{array}$ & $\begin{array}{l}\text { Safety is left to the discretion } \\
\text { of the professional who is } \\
\text { thought to have the knowledge } \\
\text { and ability to make skilled } \\
\text { independent judgements of } \\
\text { situations. In extremis, safety is } \\
\text { left to expert safety managers. }\end{array}$ \\
\hline $\begin{array}{l}\text { Corporation } \\
\text { order }\end{array}$ & $\begin{array}{l}\text { Priority is given to } \\
\text { predictability and compliance } \\
\text { to those higher in the hierarchy. } \\
\text { Values are hierarchy, control } \\
\text { and routines. } \\
\text { - Success is following routines } \\
\text { that are defined within the } \\
\text { corporation and increased } \\
\text { hierarchical status. }\end{array}$ & $\begin{array}{l}\text { Safety is secured through } \\
\text { following rules and procedures. } \\
\text { Compliance with rules and } \\
\text { procedures is controlled top- } \\
\text { down by managers through } \\
\text { hierarchical and formal } \\
\text { structures. The hierarchical } \\
\text { nature of the corporation also } \\
\text { entrusts managers with a } \\
\text { responsibility they have to take. }\end{array}$ \\
\hline
\end{tabular}


Table 4.1 (continuation)

\begin{tabular}{|c|c|c|}
\hline Logic & $\begin{array}{c}\text { Defining characteristics for } \\
\text { rationality }\end{array}$ & $\begin{array}{c}\text { Application to occupational } \\
\text { safety }\end{array}$ \\
\hline State order & $\begin{array}{l}\text { Priority is given to deliberation } \\
\text { and following the rules that } \\
\text { come from voting. } \\
\text { - Values are debate and } \\
\text { consensus, democracy, and } \\
\text { rules. } \\
\text { - Success is predictability from } \\
\text { rules, defined and followed by } \\
\text { committed people. }\end{array}$ & $\begin{array}{l}\text { Safety is secured through } \\
\text { safety legislation, designed and } \\
\text { imposed by (representatives } \\
\text { of) the state who are elected } \\
\text { through democratic majority. }\end{array}$ \\
\hline $\begin{array}{l}\text { Family } \\
\text { order }\end{array}$ & $\begin{array}{l}\text { - Priority is given to obligation to } \\
\text { the family. } \\
\text { - Values are closeness, warmth, } \\
\text { caretaking, obligation. } \\
\text { - Success is following established } \\
\text { rituals and taking care of each } \\
\text { other, and continuation of the } \\
\text { family line. }\end{array}$ & $\begin{array}{l}\text { Safety is important for the } \\
\text { continuation of the family. Safety } \\
\text { is secured through orders that } \\
\text { are based on established rituals } \\
\text { and tradition, irrespective of } \\
\text { changes in society. }\end{array}$ \\
\hline $\begin{array}{l}\text { Community } \\
\text { order }\end{array}$ & $\begin{array}{l}\text { Priority is given to a good } \\
\text { balance between social and } \\
\text { economic aspects of work. } \\
\text { - Values are community, } \\
\text { balancing, deliberation. } \\
\text { - Success is collaboration and } \\
\text { welfare. }\end{array}$ & $\begin{array}{l}\text { Safety is considered a communal } \\
\text { good and is accomplished } \\
\text { through taking care of your } \\
\text { fellow members. }\end{array}$ \\
\hline $\begin{array}{l}\text { Religion } \\
\text { order }\end{array}$ & $\begin{array}{l}\text { Priority is given to submission } \\
\text { to faith. } \\
\text { Values are obedience, morality, } \\
\text { and the order of creation. } \\
\text { - Success is harmony with the } \\
\text { creator. }\end{array}$ & $\begin{array}{l}\text { Safety is best guaranteed } \\
\text { when following the exegesis of } \\
\text { the faith, but is rooted in the } \\
\text { acceptance of fate (i.e., when it } \\
\text { costs, it costs). }\end{array}$ \\
\hline
\end{tabular}




\subsection{Method}

The data reported in this manuscript were collected as part of a larger project regarding the role of occupational safety in managers' work. In the current analyses and report, the focus is exclusively on data concerning institutional logics and logic multiplicity in the occupational safety context.

\subsubsection{Participants}

Interviews were conducted with twenty-two supervisors (all male) that work for an organisation that engages in railroad construction and maintenance. Within this organisation, occupational safety plays a prominent role in the planning and execution of work. Supervisors were explicitly targeted, as they are seen as 'the primary carriers of the contending conceptions of control' (Thornton \& Ocasio, 2008, p. 102), are likely to have the power 'to determine organizational responses to multiple institutional logics' (Greenwood et al., 2011, p. 344), and play an increasingly important role in the delegation of job tasks, management of subordinate performance, and balancing competing production, quality, and safety demands (Michael et al., 2006).

Participants were selected via a two-step procedure. An employee satisfaction survey conducted earlier by the organisation inventoried employees' willingness to participate in safety-related follow-up research. From those employees willing to participate in additional research, a selection was made by the HR manager that mirrored the managerial structure of the organisation. Potential participants $(N=30)$ were first approached via e-mail, resulting in a response rate of $73 \%$. All participants were male and at least 40 years old $(M=51.7, S D=5.3)$. Participants' tenure with the current company ranged from 3 to 37 years $(M=18.8, S D=9.1)$, and their overall tenure in the railroad construction and maintenance industry ranged from 3 to 38 years $(M=24.1$, $S D=9.4)$.

\subsubsection{Instrument}

Qualitative methods are widely used and have much potential for studying institutional logics (Reay \& Jones, 2016). Data were therefore collected via face-to-face interviews held in offices located at or nearby work locations, and 
all interviews were recorded with a digital voice recorder with consent. The semi-structured interviews covered themes related to participants' work (e.g., 'Can you tell me about the day-to-day activities in your work?' and 'How many people do you supervise?'), the role of safety in their work (e.g., 'How does safety play a role in your work?') and potential conflicting demands between safety and other organisational demands (e.g., 'Do you ever experience tensions between safety and other organisational demands?' and 'Does it ever happen that other demands get prioritised over occupational safety?'). The interviews lasted between 40 and 96 minutes, with an average duration of 62 minutes.

\subsubsection{Data analysis}

The recordings were transcribed verbatim, resulting in 314 pages of singlespaced text. The transcripts were coded using the ATLAS.ti software package (version 7.5). We used a deductive multi-step content-analytic procedure to analyse the data.

First, the first author read the transcripts and marked meaningful units of analyses consisting of single or multiple sentences. Second, this author took the lead in proposing a categorisation of the units of analyses into the institutional orders. Here, a single unit of analysis could reflect a singular logic or be reflective of logic multiplicity. Third, the proposed categorisation was discussed in iterative rounds with the entire research team, linked to the theory, and modified until agreement about the interpretation of each of the different units of analyses was reached. Three logics were found to be most prominently present: the logics of the market ( 26 quotes by 11 interviewees), the profession ( 34 quotes by 16 interviewees), and the corporation (28 quotes by 12 interviewees). Additionally, six quotes that reflected the state logic and one quote that reflected the community logic were found. There were no quotes that reflected the logics of the family or religion, which is in line with earlier findings from Johansen and Waldorf (2015) that the state, market, profession, and corporation orders are mainstream organisational-capitalist orders.

As we searched for strong patterns of institutional logics in the data, the decision was made to exclude statements referring to the community and state logics. For the community logic, this was done because there was only one 
reference present in the data (i.e., And, that is why I say [...] you go out with the four of you, and all four of you have to feel responsible. Because then you can get the job done. And, they also have to help each other because otherwise things are not going well.' - S10). For the state logic, this was done because there were only six references present in the data. Additionally, in discussing this categorisation, it proved difficult to interpret the references to the state logic unequivocally due to uncertainty about the origins of certain rules and regulations (i.e., state or corporation). For example, one interviewee stated that 'officially', he was not allowed to walk from the level crossing to the workplace without the presence of a 'safety man'. From this quote, it is not clear whether 'officially' refers to corporate policy or to state rules. Finally, in the proposed categorisation, ten units of analyses from five interviewees that reflected logic multiplicity, wherein the logics of the state, market, profession, and corporation were present, were found. Five quotes that reflected logic multiplicity but included a (possible) reference to the state logic were excluded from further analyses, leaving five quotes that the authors agreed reflected logic multiplicity.

\subsection{Results}

The analysis identified three logics in the data: the logics of the market, the profession, and the corporation. First, the emergence of each of these logics will be illustrated and explained (4.4.1). Second, the presence of logic multiplicity and conflicting logics will be analysed and presented (4.4.2).

\subsubsection{Institutional logics}

The results illustrated that interviewees justified their safety-related behaviours using various logics. Interviewees' justifications of safety-related behaviours were not only rooted in different institutional orders but also accounted for using arguments that could be linked to the repertoire of elemental categories within these orders (see Thornton et al., 2013).

\section{Logic of the market}

Interviewees' rationalisations of safety-related behaviours and decisions reflected the logic of the market. For example, interviewees referred to the 
system put in place by the public contracting authority (the NEN Safety Culture ladder) to promote occupational safety through offering economic advantages. Within this system, organisations can obtain a fictitious discount within public bids if their safety performance meets certain standards. The higher the safety standards, the higher the fictitious discount, thus increasing the chance of winning the bid. Employees use that financial stimulus as a motivation to work safely:

'That [fictitious] discount by [the public contracting authority], it increases our chances, it increases our continuity; loosely translated, it means that I can keep my job. They will not all think like that but..., so that drive is pretty inherent. People have started to think very commercially, to put it bluntly: "we have to do it safely because then we get a discount"' - M20, Higher-level manager

This example exemplifies the logic of the market: safety performance is considered an asset for gaining market share, as better safety performance leads to increased consumer preference. The market logic was also reflected through the market focus on increased efficiency profit, as rail contractors face heavy competition and are willing to accept the same work for much less than before:

'For example, the contract [area], we accepted that contract for $60 \%$ of the budget we used to get. So, all offerings are brought on the market for about half of their value. And, that really leads to a competition between contractors, which is not good. Because, as foreman, you are responsible for both safety and production. And, that is a balance that you have to keep.' - M10, Foreman

The foreman expresses his worries regarding the negative effects that the competition between contractors has on the balance between safety and production. Albeit implicit, he is worried that the focus on increasing profitability will lead to a focus on production which in turn will result in less safety.

Logic of the profession

Inherent to a profession are certain standards, and interviewees described 
the influence of such standards in their work when it comes to occupational safety. Interviewees stated that they make their own decisions in work and that they use their professional expertise to prioritise occupational safety over conflicting organisational demands:

'Well, you know for yourself that you can perform the work safely. I myself know what I do, and I do it safely. Look, if it really cannot be done, then you will say: "scr*w you"' - M24, Group leader

Interviewees also expressed that they prioritised work over following standard working procedures that are put in place to ensure the safety of employees. Here, quality of craft and personal reputation are associated with 'getting the work done':

'People want to get started with their work. That is it. It is only a waste of time when you are waiting there. [...] But, you know, I would walk to the workplace too. Even if I had more time. Because you can always have a setback you know? And, then, you need that time; you just really need that time' - M31, Group leader

These quotes are reflective of the manner in which professionals use their expert knowledge and ability to make skilled and independent judgements about what is safe and what is not.

\section{Logic of the corporation}

The institutional logic of the corporation is centred around the organisational structure of the organisation with its hierarchical levels. The influence of managers' hierarchical power on safety-related decisions and occupational safety was visible in the data. Managers used their hierarchical power to unburden their subordinates. One of the interviewees described a situation in which his department reached its maximum capacity in terms of occupancy of machinery:

'Well, then I called the director of projects on Friday afternoon and the manager of another one of our branches and I told them: Guys, we have reached our maximum capacity and we are all out. If we have to choose which one of you to cancel this weekend due to 
sickness of a driver or for some other reason, let's agree that I will call you. My men know that they can call me, and if the moment comes that we have to make decisions, then we can call with the three of us, and we decide on what to do.' - M16, Higher-level manager

When managers take the responsibility they are entrusted with, they help their employees make safe choices. Employees do not have to deal with higher managers that they do not know and might be more willing to choose safety over the fear of negative consequences. However, managers also used their hierarchical power to determine the content and planning of work by requesting actions from their employees that conflicted with safety but could be beneficial for the market position of the firm:

I notice sometimes that I ask my employees, what I call "immoral questions". For example, safety is very important, but can't we cancel that safety appointment and focus on our enterprise resource planning?' - M20, Higher-level manager

Although they were aware of the pressure that their managerial position can exercise:

'So, I have to be careful myself, with what I say and the way I say it. Because, if those boys think that they have to do this, have to do that, that things should be completed, then you put a certain pressure on them.' - M4, Foreman

As demonstrated above, examples of rationalisations rooted in the institutional orders of the market, profession, and corporation were apparent in the data. Table 4.2 provides more examples of such rationalisations.

\subsubsection{Logic multiplicity}

The previous section already demonstrated that multiple logics are core to occupational safety. In addition to these examples, which each reflect a single logic, examples that reflected complexity that arose when incompatible prescriptions from multiple institutional logics were encountered also emerged in the data. Examples of complexity between the institutional 
Table 4.2

Safety rationale per institutional logic.

\begin{tabular}{|c|c|c|}
\hline Order & Rationale & Example \\
\hline Market & $\begin{array}{l}\text { Efficiency underlies } \\
\text { profitability. } \\
\text { Business practices } \\
\text { are determined by the } \\
\text { maximum value possible } \\
\text { from transactions. } \\
\text { - Self-interest of the client. }\end{array}$ & $\begin{array}{l}\text { 'Yes, but [Name interviewee], later } \\
\text { on, it will be about the money, and } \\
\text { then what? Then, do we still talk } \\
\text { about safety?' - M17 } \\
\text { 'But, if there are incidents, } \\
\text { unforeseen circumstances, we think } \\
\text { that [the client] should take their } \\
\text { responsibility. And, they do not, and } \\
\text { that's a shame. Fortunately we are } \\
\text { a big company, and we can suffer } \\
\text { that, but let's be honest, if you are a } \\
\text { smaller company you cannot simply } \\
\text { lose } 100.000 \text { euro. Then, you end up } \\
\text { in a difficult situation.' - M13 }\end{array}$ \\
\hline Profession & $\begin{array}{l}\text { - Safety is left to the } \\
\text { discretion of the } \\
\text { professional who is thought } \\
\text { to have the knowledge } \\
\text { and ability to make skilled } \\
\text { independent judgements of } \\
\text { situations. } \\
\text { The organisation structure } \\
\text { of the professional is based } \\
\text { on individual relations. } \\
\text { Sources of authority: } \\
\text { professional association. }\end{array}$ & $\begin{array}{l}\text { 'Actually, I should have said that it } \\
\text { would have been better to cancel the } \\
\text { work because it did not feel right to } \\
\text { me.' - M5 } \\
\text { 'I mean, if they haven't made the } \\
\text { proper arrangements, then it's } \\
\text { their bad luck. [...] Five days later, } \\
\text { the same person has not made the } \\
\text { proper arrangements. Then, I say } \\
\text { “Good friend, you had five days to } \\
\text { arrange it, you haven't done so, and } \\
\text { now you want me to do the work } \\
\text { illegally." Well, I won't do that.' - } \\
\text { M33 }\end{array}$ \\
\hline
\end{tabular}


Table 4.2 (continuation)

\begin{tabular}{|c|c|c|}
\hline Order & Rationale & Example \\
\hline Corporation & $\begin{array}{l}\text { - The informal control } \\
\text { mechanism, the } \\
\text { organisation's culture, is } \\
\text { changing. } \\
\text { - Hierarchy comes with } \\
\text { power to control but also } \\
\text { with responsibility. }\end{array}$ & $\begin{array}{l}\text { 'And, now, they should come to } \\
\text { understand that our management } \\
\text { is now stepping away from it. } \\
\text { Previously, they sometimes stated: } \\
\text { "You have to work safely", but they } \\
\text { did come to you like: "Can you work } \\
\text { for } 18 \text { hours?" But, that is no longer } \\
\text { the case. That is done. And, luckily, } \\
\text { you now notice with 80\% of the } \\
\text { management that that is over.'- } \\
\text { M17 } \\
\text { [Name former manager] did not } \\
\text { have such a stance. He was more } \\
\text { like "Yes, that is your problem", } \\
\text { something like that, along those } \\
\text { lines. He thought safety to be } \\
\text { important but he directed a lot } \\
\text { of ownership to everyone. And, } \\
\text { fortunately, [name current } \\
\text { manager] is a manager who says: } \\
\text { "Okay, that is my problem, we will } \\
\text { solve that together."' - M20 }\end{array}$ \\
\hline
\end{tabular}


orders of the market, profession, and corporation were present (see Figure 4.1). Within each quote, the part referring to a particular logic is underlined. Words or sentences referring to the market logic are underlined with a thick line, those referring to the profession logic are underlined with a dotted line, and those words and sentences referring to the corporation logic are double underlined.

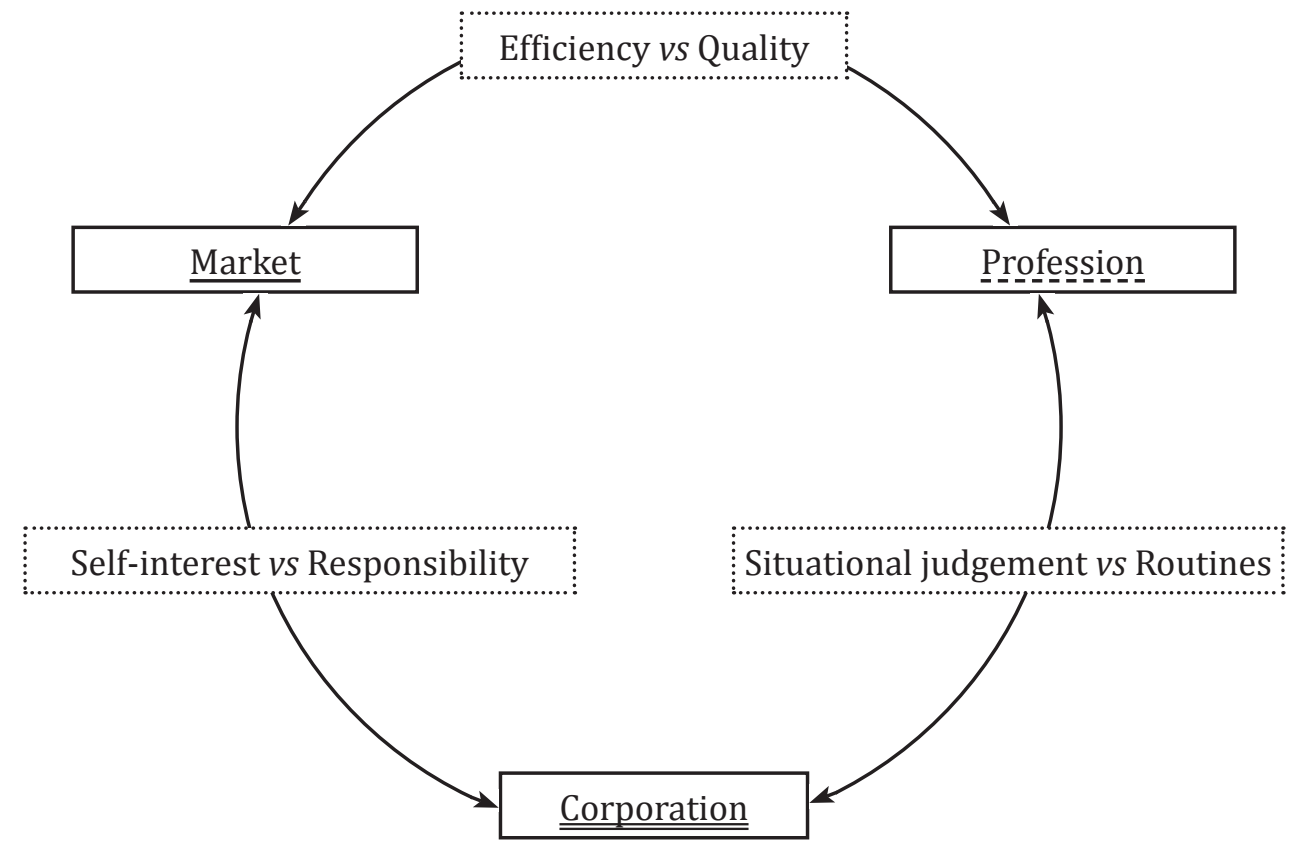

Figure 4.1. The institutional orders of the market, profession and corporation and the complexities arising from incompatible prescriptions between them.

Logic of the market versus logic of the profession

When the orders of the market and the profession collide, actors face the market logic's self-interested focus on increasing (efficiency) profits and the profession logic's focus on delivering high-quality work and personal expertise.

'This year, we have cancelled our bids three times. [Researcher: Yes, but I assume there are just so many times you can cancel your bids 
on work?] [...] Even if we have to do it a hundred times, that does not matter. I will not make concessions to work when the electricity. is running. I just do not. Very simple; we simply do not. And, that is also something that the customer has to learn. And, if that means that our organisation does not have any work in the Netherlands for a while, but you have to be able to explain it as management.' M12, Higher-level manager

In the above quote, the professional is not willing to make concessions to working safely. Based on his knowledge, he judges that working when electricity is running is unsafe, despite the apparent wish of the client. The interviewee in this example solves the complexity arising from the collision of the logic of the market and the logic of the profession by stating that the client (market) has to learn that the professional will not make concession to working safely. He implicitly stresses the legitimacy of his choice based on his expert knowledge about work safety.

\section{Logic of the market versus logic of the corporation}

When the market logic collides with the logic of the corporation, actors face the market's self-interested focus on increasing (efficiency) profits, while at the same time, they have to uphold their status in the managerial hierarchy and their bureaucratic roles, and they must think about the market position of the firm:

'A few years ago, we sometimes thought we just bid on a work and then we will see what happens; we pressure the customer; it is his problem. Well, the customer responded "you have accepted the job, it is your problem". [...] I am responsible for the people that work here; well, a safe workplace must be present; we must have validated that in the tender phase. If not, we are going to question it, and if that question is answered unsatisfactorily, we will not make a bid on that work.' - M12, Higher-level manager

The interviewee describes that initially, he was more motivated by increasing efficiency, but when confronted with the self-interest of the client reasoning from the same market logic, he turned to the corporation logic and took responsibility for his subordinates. This example illustrates that the 
interviewee tried to solve the complexity initially by leaving things implicit, reckoning that the client would take responsibility. However, when the safety question was made more explicit, complexity arose, and in this instance, the parties involved were forced to settle the responsibility question. As the client refused to pick up the glove, the interviewee was almost forced to do so.

\section{Logic of the profession versus logic of the corporation}

When the order of the profession collides with the order of the corporation, actors are confronted with the managerial hierarchy and the organisational culture (corporation logic) on one hand and personal expertise and reputation (profession logic) on the other:

'Well, we solve a lot of things ourselves here, and that is something we shouldn't do. You try to have a solution-focussed stance and sometimes you do things that are not possible. And when you don't do_[solve] them, you are a dick, but if you do [solve] them and something happens, you are screwed. And, those are seemingly very small things, but they can have far-reaching consequences' - M24, Group leader

The interviewee describes that - following from the logic of the profession - there is a certain willingness to get the work done, which is also expected from them. However, getting the work done requires professionals to use their expert knowledge to make a translation from the general applicable rules and routines of the corporation to an approach that fits with the specific situation. In the process of doing so, they are likely to deviate from and violate those corporate routines, and inherently the corporation logic. Making this translation is accepted - and perhaps even expected by managers - but only as long as things go well. When things go wrong, managers will use their hierarchical power to protect themselves, hiding behind corporate routines that should have been followed.

\subsection{Discussion}

The aim of this study was to increase our understanding of what drives safety-related decisions and behaviours. We proposed that the discourse of 
institutional logics can be used as a lens to uncover, illustrate, and explain rationales for safety-related behaviours and decisions that are otherwise left uncovered or dismissed as irrational (cf. Hopkins, 1999; Reader \& O'Connor, 2013; Swuste et al., 2010). The fictitious example from the introduction already highlighted that people can have different rationales, leading to action ('Being familiar with the worksite and the dangers associated with not wearing a helmet on site, George grabs a spare helmet and gives it to the visiting employee') or inaction (e.g., 'Peter remembers the client stressing the importance of finishing the work in time, and he decides not to interfere as this might negatively affect the work pace').

\subsubsection{Contributions}

This study makes several contributions to the field of occupational safety. First, it demonstrates that an institutional logics perspective can be effectively applied to empirical data and used to uncover and identify the easily overlooked differences between the rationales that people employ to justify their views about occupational safety. By applying the institutional logics perspective to the descriptions of safety issues by managers in a railroad construction and maintenance organisation, we were able to uncover the logics of the market, profession, and corporation. Within this study, the market logic was reflected in the use of safety performance as a means to increase consumer preference and the effects of market competitiveness on safety. The professional logic led managers to prioritise staying safe over getting the work done, whereas others prioritised the timely completion of work over standard operating procedures that should keep them free from harm. The logic of the corporation was reflected in the means in which managers made use of their hierarchical power to determine the content and planning of work. These examples demonstrate that an institutional logics perspective can be used to identify different rationalities underlying behaviours and decisions and how those different logics lead to differences in approaches towards occupational safety.

Second, in addition to illustrations of individual institutional logics in the data, examples of logic multiplicity between the logics of the market, profession and corporation emerged. Here, people use the same words but speak different languages. Complexity between the logics of the market and the profession arose due to the incompatibility of the market logic's self-interested focus on 
increasing (efficiency) profits, whereas the professional wanted to adhere to high-quality work standards. In this situation, the manager resolved the conflict by stating the client had to learn from the professional's personal expertise regarding how to keep the work safe. The complexity between the logics of the market and the corporation occurred as result of the market logic's self-interested focus on increasing (efficiency) profits, whereas managers have to uphold their hierarchical status and responsibilities. Initially, the solution to complexity was sought in leaving the responsibility for occupational safety implicit. However, when the client made this issue explicit and refused to take responsibility, the manager was forced to do so. The complexity between the logics of profession and the corporation arose as a result of rules and routines that are prescribed by the corporation that were incompatible with the work that was expected to be done. The group lead tried to solve the complexity by using his expert knowledge to translate the general rules and routines to fit with the specific situation.

The new concepts (i.e., the various logics and their associated elemental categories) introduced by the institutional logics framework provide both researchers and practitioners with a discourse that allows them to study and analyse occupational safety in new manners. These concepts not only allow for providing meaning to seemingly irrational behaviours (Hopkins, 1999) but also provide a means to break down the complex structure of behaviours rooted in various rationales into manageable pieces. Additionally, they complement the local rationality principle that Dekker (2003) introduced to explain human error and further it by illustrating that similar people in similar situations can reach different conclusions based on the prevailing institutional logic.

Third, given that organisations are likely to encounter logic multiplicity, the framework designed Besharov and Smith (2014), as discussed in the Introduction, provides an indication of the degree of internal conflict. The organisation in the current study was categorised as an organisation that is characterised by high logic centrality and low logic compatibility in terms of occupational safety. The results illustrate not only that multiple logics are core to safety-related decisions and behaviours (i.e., a high degree of centrality) but also that these logics provide contradictory prescriptions for action (i.e., a low degree compatibility). In line with the results, this implies that members of the organisation 'hold competing expectations about appropriate organisational 
goals and lacking a clear guideline as to which goals should prevail' and that the organisation encounters extensive conflict (Besharov \& Smith, 2014, p. 371). The contradictory prescriptions for action and the resulting competition between organisational goals jeopardises occupational safety. If occupational safety is not beyond a doubt the number one organisational goal, employees cannot be expected to maintain workplace safety at all times. The abilities to predict the degree of conflict within an organisation and to understand how this conflict originates provide managers with opportunities to minimise such conflict and its impact on performance and stability (Besharov \& Smith, 2014). In sum, the demonstrated usability of the institutional logics perspective in studying and analysing occupational safety provides both researchers and practitioners with new means to address human and organisational occupational safety issues.

\subsubsection{Practical implications}

The demonstrated usability of an institutional logics perspective for occupational safety also has implications for practice. First, managers should be aware that people can have different underlying rationales for their safety-related thinking, decisions and behaviours. Second, in addition to this awareness, the ability to identify the different logics at play allows managers and safety professionals to minimise the impact of speed on accuracy in times of haste and crisis. Third, through awareness and identification of the different logics, managers and safety professionals can help in exploring misunderstandings in conversations between different actors, in addition to uncovering the nature of these misunderstandings through articulating the various logics. Fourth, the discourse of logics can be used in the analysis of occupational safety incidents. As Dekker (2003) notes, no one comes to work to do a bad job, and what they do and decide makes sense to them in that specific situation. In the case of a safety incident, the institutional logics perspective can shed light on the motivations and rationales that what drove employee decisions and behaviours instead of dismissing the accident solely as caused by human failure. Finally, the discourse of logics provides (safety) managers with the ability to recognise, articulate, and discuss the different rationales for occupational safety that are easily overlooked by traditional management approaches. In doing so, it not only contributes to the understanding of employee functioning in terms of occupational safety but also provides managers with opportunities to engage with employees 
by articulating the divergent rationales they apply and to use the discourse of logics by adapting their arguments in such a manner that they are in line with the dominant logic of their conversation partners, thus increasing their intelligibility and persuasiveness.

\subsubsection{Limitations}

Although we are convinced that the findings presented in this study contribute to the current knowledge and understanding of safety-related decisions and behaviours, there are some limitations that must be addressed. First, the group of managers participating in this study was limited in size, especially considering the limited number of managers that made implicit references to institutional logics. Future research should therefore further explore the presence of (conflicting) institutional logics concerning occupational safety with larger research samples and in organisations operating in other sectors. Second, although the state logic seemed to emerge from the data, it proved difficult to unequivocally interpret these units of analysis. The wordings used by interviewees did not clearly allow for a definite answer regarding the origins of the referenced rules (i.e., state or corporation), which led us to adopt a rather conservative approach and exclude all related quotes. Future research should therefor explore whether references to the state logic can be interpreted more clearly.

\subsection{Conclusion}

The current study demonstrated that the institutional logics perspective is a valuable lens that provides new methods to uncover, study, and explain the different rationales underlying occupational safety. It allowed us to uncover the logics of the market, profession and corporation in occupational safetyrelated thinking, decisions and behaviours of managers. Such insights into and understanding of the various rationales underlying safety can help in the prevention and analysis of accidents, ultimately leading to safer workplaces.

\section{Acknowledgements}

This research did not receive any specific grant from funding agencies in the public, commercial, or not-for-profit sectors. 




\title{
Chapter 5
}

\section{Enabling employees to work safely: The influence of motivation and ability in the design of safety instructions}

\author{
'There are three kinds of men. The one that learns by \\ reading. The few who learn by observation. The rest of \\ them have to pee on the electric fence for themselves.'
}

Will Rogers

Cornelissen, P.A., Van Hoof, J.J., \& Van Vuuren, H.A. (2014). Enabling employees to work safely: The influence of motivation and ability in the design of safety instructions. Technical Communication, 61(4), 232-244. 


\subsection{Introduction}

Worldwide, every minute, four workers die from a work-related accident or disease, and every minute, 640 workers have a work-related accident (International Labour Organization [ILO], 2013). Extrapolating those numbers leaves us with 5,760 occupational deaths per day, and 2.1 million work-related deaths per year worldwide. According to the International Labour Organization (ILO), any deaths, diseases, or injuries resulting from an occupational accident are classified as an injury. Comparably, ILO (2014) defines an occupational disease as 'a disease contracted as a result of an exposure over a period of time to risk factors arising from work activity, and an occupational accident as 'an unexpected and unplanned occurrence, including acts of violence, arising out of or in connection with work which results in one or more workers incurring a personal injury, disease or death' (ILO, 2014).

Contrary to popular beliefs, occupational injuries are not merely an immense problem in developing countries, but also in the developed countries. In the Netherlands, for example, nearly 450 thousand workers (on a total working population of around 7 million) were injured on the job in 2013 (Statistics Netherlands, 2014). Around half of these injured workers missed out one day of work, and over a third of them missed over four workdays. These numbers have been relatively stable since 2005 (Statistics Netherlands, 2014), which indicates an ongoing need for a further increase in workplace safety. Apart from the emotional toll of work-related accidents on the injured workers, their families and colleagues, the cost of these accidents equals an estimated $4 \%$ of the global gross domestic product each year (International Labour Organization, 2010).

Nowadays, most of the bigger companies in risk industries have instituted an Environment, Health and Safety (EHS) department; a department charged to secure and improve the company's environmental, (employee) health, and (employee) safety efforts, such as assessing risks, reducing waste, and improving work conditions for employees by introducing ergonomic workstations. The matter is becoming increasingly important with increased external EHS regulations and government control (Cahill, 2010; Hofmann et al., 1995). The importance of EHS for the public image and corporate risk 
profile is increasing (Chinander, Kleindorfer, \& Kunreuther, 1998; Hasan \& Jha, 2013). Organisations could benefit from these measures in additional ways as research has associated workplace safety with both organisational climate (e.g., Neal et al., 2000) and job satisfaction (Gyekye, 2005), which are both important for employee well-being. Combining the organisational, financial, and emotional costs of workplace accidents provides a societal relevance of the topic: There is a lot to gain from focusing on safety.

In an attempt to find a conclusive solution to safety, researchers have focused on demographics like age and tenure (Mearns et al., 2003; Gyekye \& Salminen, 2009), and behavioural aspects of workers and management (e.g., Cheng, Lue, Lin, \& Fan, 2010; Margolis, 1973 as cited by Coyle et al., 1995; Jonson, 1982 as cited by Coyle, Sleeman, \& Adams, 1995; Unsar \& Sut, 2009).

Addressing safety through behaviour can be advantageous to organisations, since major modifications to the building, machinery, or equipment are not necessary (Swuste et al., 2014). Creating safe environments can be achieved in two ways; first, through adjustment of employee behaviours (e.g., altering standard operating procedures, or training) (Swuste et al., 2010), and second, through (e.g., designing out hazards, guarding of hazards, and warning employees) (Ponnet, Reniers, \& Kempeneers, 2015).

Compared to adapting machinery, equipment, and/or the building, creating effective safety instructions which explain appropriate employee behaviours can be a relatively easy and cheap way to increase safety in the work environment. Safety instructions are one of the cornerstones of the risk control system, being the translation from top management commitments into specific details (Hale \& Borys, 2013). Safety instructions are instituted to specify, communicate, and control safe behaviour among employees in dangerous situations, and to prevent accidents from happening (Elling, 1997; Hale, 1990; Herrerro, Saldaña, Del Campo, \& Ritzel, 2002). Designing effective safety instructions is complex, and all too often, these are deemed to be too strongly focused on technical requirements (Herrero et al., 2002), to be unrealistic (Hale \& Borys, 2013), or to solely serve the interests of the organisation (Elling, 1997). Another problem with safety instructions is that they can be misinterpreted, resulting in unsafe behaviours (Racicot \& Wogalter, 1995). So, while safety instructions can be beneficial, their design is not without problems. This is especially important in high-risk work 
environments where even the slightest problems with safety instructions can become catastrophic. In this study, we focus on motivation and ability attitudes of warehouse employees, to provide insight in how to write effective safety instructions for high-risk work environments.

In late 2013, we had the opportunity to explore the extent to which the sublevels personal motivation and external motivation, as well as personal ability and external ability, affect safety climate and safety performance.

\subsubsection{Workplace safety}

According to the International Labour Organization and the World Health Organization, workplace safety -or occupational health- should aim at:

...the promotion and maintenance of the highest degree of physical, mental and social well-being of workers in all occupations; the prevention amongst workers of departures from health caused by their working conditions; the protection of workers in their employment from risks resulting from factors adverse to health; the placing and maintenance of the worker in an occupational environment adapted to his physiological and psychological capabilities and; to summarise: the adaptation of work to man and of each man to his job. (International Labour Organization, 1998, p. 21)

Many researchers have tried to find a way to positively affect workplace safety. In their studies, safety performance was often perceived as an indicator of workplace safety. However, little consensus exists on which approach is the right one to increase the safety performance of employees.

\subsubsection{Safety climate and safety culture}

Workplace safety refers to actual, measurable employee well-being, but workplace safety also has a more perceptual side to it. Safety climate and safety culture are parts of this perceptual side of workplace safety. Safety climate consists of shared beliefs (i.e., perceptions) of safety in the workplace (Díaz \& Cabrera, 1997; Gyekye \& Salminen, 2009). These shared perceptions are also referred to as perceptions of workplace safety. 
The concept safety climate is often wrongly used as a synonym of safety culture (Cox \& Flin, 1998; Yule, 2003). Yule (2003) looked into both concepts from a conceptual and theoretical point of view and found that, in line with the work of others (Cox \& Flin, 1998; Guldenmund, 2000), the two concepts refer to different levels. Although the concepts share elements, these might not be reflective of a unitary concept, but rather work as independent concepts that complement each other (Yule, 2003).

Safety culture has to be viewed as a sub-facet of organisational culture, and exists at a higher level of abstraction than safety climate. It is characterised by 'shared underlying beliefs, values, and attitudes towards work and the organisation in general' (Yule, 2003, p. 3), whereas safety climate appears to be closer to operations and is 'characterised by day-to-day perceptions towards the working environment and practices, organisational policies, and management' (Yule, 2003, p. 3). Perceptions of workplace safety are important for actual occupational safety as they are associated with variables relating to industrial accident rates and levels of job-related anxiety, stress, and exposure to environmental hazards (Hayes, Perander, Smecko, \& Trask, 1998). Perceptions of workplace safety are indicators of safety performance (Cooper \& Philips, 2004; Gyekye \& Salminen, 2009; Hayes et al., 1998; Neal \& Griffin, 2006). The latter seems to be partially affected by safety knowledge and motivation (Zhou, Fang, \& Wang, 2008, p. 1410). The combined findings, which are in line with a variety of sources (e.g., DeJoy et al., 2004; Guldenmund, 2000; Gyekye \& Salminen, 2009; Jiang, Yu, Li, \& Li, 2010; Neal et al., 2000; Pousette, Larsson, \& Törner, 2008; Silva, Lima, \& Baptista, 2004), lead to the hypothesis (H1) that higher levels of perceptions of workplace safety (i.e., safety climate) will be accompanied by higher levels of safety performance as depicted in Figure 5.1.

As the literature shows, the solution to workplace safety has been sought from a variety of angles. The idea that motivation and ability are responsible for performance dates back to 1955, when Maier (as cited in Burke, Sarpy, Tesluk, \& Smith-Crowe, 2002) specified motivation and ability as determinants of job performance. Since then, this idea gained a lot of attention in research (Burke et al., 2002), with mixed results. Campbell et al. (1993, as cited in Neal et al., 2000) stated that only three determinants are responsible for individual differences in safety performance: motivation, skill, and knowledge. These 
$\mathrm{H} 3$

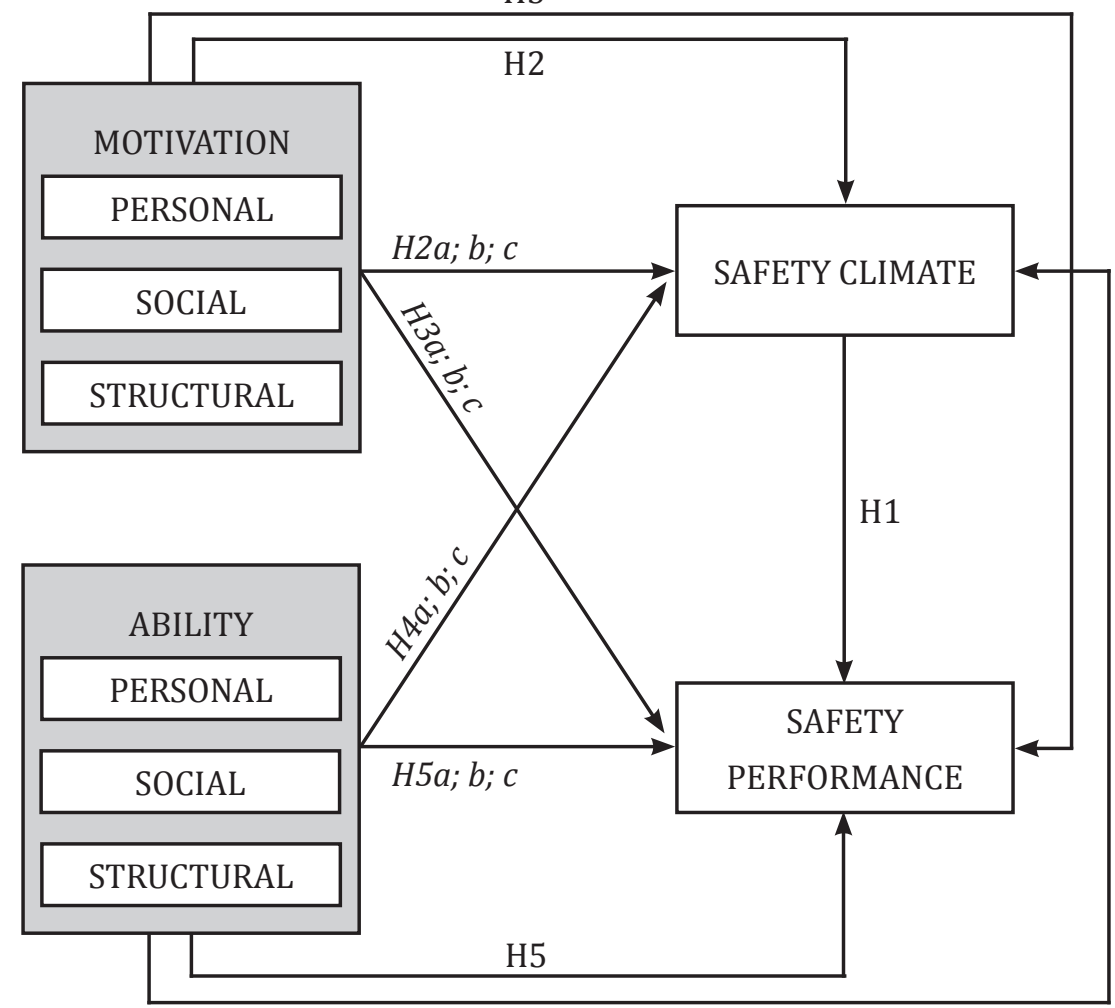

$\mathrm{H} 4$

Figure 5.1. Hypothesised influence of three levels of motivation and ability on safety climate and safety performance

findings, combined with the findings by Zhou et al. (2008), lead to the belief that the proposed classification (i.e., motivation and ability) is the right choice to approach the matter of workplace safety. Additionally, research by Hofmann et al. (1995) provides leads that indicate the usability of viewing motivation on different levels (e.g., individual, micro, and macro). Below, we will elaborate on the concepts motivation and ability, and extend the different motivational levels as indicated by Hofmann et al. (1995) to ability. 


\subsubsection{Motivation}

Motivation has been equated with behavioural intention (Sheeran, 2002), although the Theory of Planned Behavior (TBP) (Ajzen, 1991) shows that social norms and perceived control also play a role in people's behavioural intention and actual behaviour. In general, people will assume that safety behaviour is a very easy choice: Not acting in a safe way will increase the risk of getting involved in an accident. Andriessen describes motivation as 'the result of a -not necessarily conscious- decision making process, in which a person weights the advantages and disadvantages of different acts (different kinds of work behaviour) and then chooses the behaviour with the best yield (that is, he is then motivated to that act)' (Andriessen, 1987, p. 368). It is therefore hypothesised that higher levels of motivation will lead to higher levels of safety climate (H2) and safety performance (H3). However, other levels of motivation play a role as well. Consider the following situational factors: 'the esteem of colleagues (because you're seen as tough or because you are not childish), the esteem of the supervisor (because you don't hold up the work), and more convenience (because you don't constantly have to be on your toes)' (Andriessen, 1978, p. 368). These all produce counterweight to the perceived risk of accidents.

On a personal level, motivation can be viewed as the intrinsic desire or choice people make to act in a certain way. It is therefore hypothesised that higher levels of personal motivation among employees will lead to higher levels of safety climate (H2a) and safety performance (H3a).

In the early 1960s, Milgram shocked the world when he revealed the power peer pressure can exert over others in his famous Milgram experiments (for further information, see Milgram, 1963). A link with the TPB (for more information, see Ajzen, 1991) is easily made, as social motivation is similar to the subjective norm. According to the TPB, the subjective norm is based on a combination of normative beliefs -'the likelihood that important referent individuals or groups approve or disapprove of performing a given behaviour' (Ajzen, 1991, p. 159)- and a person's motivation to comply. In other words, we humans find it important what others think of us, and how they see us to such an extent that we might even change our behaviours for it. This notion also underlies the two-step flow theory (for more information see Katz, 1957) which indicates that personal contacts are considered far more important than 
direct mass media messages. It is therefore hypothesised that higher levels of social motivation will lead to higher levels of safety climate (H2b) and safety performance (H3b).

Last, a third level of motivation was proposed by Andriessen's (1978) research, in which management exerted influence through rewards and penalties (see also Hasan \& Jha, 2013). It is therefore hypothesised that higher levels of structural motivation will lead to higher levels of safety climate (H2c) and safety performance (H3c).

\subsubsection{Ability}

Ability consists of the combination of skill and knowledge people acquire through training. It is hypothesised that higher levels of ability will lead to higher levels of safety climate (H4) and safety performance (H5). Again, we hypothesise that ability consists of different levels.

Personal ability is about influencing (enhancing) the practical and theoretical know-how of individuals to enable them to perform the desired vital behaviours. Organisations (attempt to) do so by providing training and instructions to their employees. It is therefore hypothesised that higher levels of personal ability will lead to higher levels of safety climate (H4a) and safety performance (H5a).

A social level of ability is similar to social capital. Although social capital might seem a relatively new concept due to the increased attention it has been given in the last decade, it is far from new. Farr (2004) traced the term back to 1916 -when Lyda J. Hanifan supposedly used the concept for the first time- and conceptualised social capital as:

The network of associations, activities, or relations that

bind people together as a community via certain norms and psychological capacities, notably trust, which are essential for civil society and productive of future collective action or goods, in the manner of other forms of capital. (p. 9)

In laymen's terms, social capital reflects as much as: the whole is greater than the sum of the parts. In this equation, 'the whole' is the capital, often in 
forms of knowledge. The 'parts' consist of actors in a specific society or social network. It is hypothesised that higher levels of social ability will lead to higher levels of safety climate (H4b) and safety performance (H5b).

The last level of ability, structural ability, refers to nonhuman power. The main question revolves around the environment: How can it be used and transformed to enable change? Here, one can think of getting the right equipment, decorating a building, designing the physical lay-out of a building, and so on. The power that can exert from changes in the environment people work or live in has been proven in numerous fields, some more well-known than others (e.g., Sonderegger \& Sauer, 2010; Van der Woning, 2008). The inclusion of structural ability also counters criticism on the classification of safety performance as provided by Campbell et al. (1993, as cited in Neal et al., 2000), that situational factors should be taken into consideration, for these can be of influence as well (Neal et al., 2000). It is therefore hypothesised that higher levels of structural ability will lead to higher levels of safety climate (H4c) and safety performance (H5c).

\subsection{Method}

To explore the hypothesised relations between the levels of motivation and ability on the one hand and safety climate and safety performance on the other, we designed a questionnaire for employees of a Dutch high-tech and high-risk warehouse.

\subsubsection{Procedure}

All employees were approached via their (direct) supervisors, and given time off from their work to complete our questionnaire. In groups of five to ten -depending on how many employees were needed in the warehouseemployees went to a conference room which was made available for this purpose. Upon entrance, participants chose an English or a Dutch language version of the questionnaire. They were then asked to carefully read the first page, and to subsequently answer the questions. During the study, one of the researchers was continuously available for questions. The employees returned their completed questionnaires to the researcher without the involvement of supervisors or colleagues. 


\subsubsection{Participants}

Of all available warehouse employees coming into contact with high-risk products $(N=92), 77$ participated in our study. They worked at different departments in the warehouse, with activities varying from picking products to (un)loading trucks and processing incoming products. Participants were predominantly male ( $n=54)$ as opposed to female ( $n=18)$; 5 values were missing. The age of the participants ranged from 19 years old to 62 years old, with a mean of 40.2 years, while tenure ranged from 3 weeks to 9 years, with a mean of 4.8 years. Of the employees who disclosed their job situation, $53 \%$ were structural employees, $12 \%$ were employed as a contractor, and $13 \%$ had a flex-worker contract.

\subsubsection{Instrument}

Data were collected through a questionnaire consisting of 50 items, which were divided into eight different constructs, using a five-point Likert scale ranging from totally disagree to totally agree. The eight constructs are a mix of existing scales and questions designed for the specific professional environment of our study, and will be discussed here.

We used the six-item scale by Hahn and Murphy (2008) to measure safety climate. This scale showed good internal consistency before, with Cronbach's alpha coefficients between .71 and .85. An example item is: 'The health and safety of workers is a high priority with management where I work.'

We used items from several sources to measure safety performance. First, we used a shortened version of Whu, Chen and Li's (2008) scale, with reported Cronbach's alpha coefficients between .89 and .96 in earlier research. Second, we used items that were designed for our specific context, with a total of 14 items in the final scale. An example item is: 'I have not been injured in the workplace within the 12 past months.'

To measure each of the three different levels (personal, social, and structural) of motivation and ability, five items were used. An example item of personal motivation is: 'I feel motivated to behave safely in my workplace.' The instrument used by Williamson, Feyer, Cairns, and Biancotti (1997) to assess levels of personal motivation for safe behaviour, positive safety practice, risk 
justification, fatalism, and optimism was consulted to create the scales to measure motivation and ability. We rearranged 21 items from this instrument into new scales, adapted 2 items, and added 7 new items to create the scales for our study. Furthermore, at the end of the questionnaire, we added five questions to measure relevant background and professional characteristics (gender, age, work tenure, type of contract (regular employee, contractor, or flex worker), and primary department of employment). Employees were also asked to grade the company's health and safety program (ranging from 1 to 10 ), and given the opportunity to leave remarks.

The final instrument was pretested with three experts to evaluate the items' clarity and relevance. This pre-test led to some minor textual improvements. Additionally, we pretested the final questionnaire with four nonparticipating employees to confirm the comprehensibility of the items.

\subsubsection{Analysis}

We loaded the data from the 77 questionnaires into SPSS 20.0, and checked the file for errors. As a result, the data of two questionnaires were removed due to a large number of missing data. Subsequently, we recoded negative items and calculated the reliability of each construct, which resulted in a factor analysis and a regrouping and relabelling of the constructs. Finally, we conducted a multiple regression analysis to validate the research model.

\subsection{Results}

The safety climate and safety performance scales showed good internal consistency with Cronbach's alpha coefficients of .78 and .80. None of the scales intending to measure the different levels of motivation and ability (i.e., personal motivation, social motivation, structural motivation, personal ability, social ability, and structural ability) showed sufficient internal consistency (Cronbach's alpha coefficients between .12 and .59). We therefore decided to conduct Principal Components Analysis (PCA) to further investigate those data and identify the underlying structure. 


\subsubsection{Exploring the structure of motivation and ability}

We followed the method of principal components analysis to determine the structure underlying the items used to measure levels of motivation and ability. Initially, the factorability of the items was assessed using several wellrecognised criteria. With a value of .62, the 30 items intending to measure the six different variables exceeded the recommended value of .60 on the Kaiser-Meyer-Olkin test (Kaiser, 1970). Bartlett's Test of Sphericity (Bartlett, 1954) showed statistical significance for the 30 items, which supports the factorability of the correlation matrix. Principal components analysis revealed the presence of ten components with eigenvalues exceeding 1 , together explaining $74.4 \%$ of the total variance. An inspection of the scree plot revealed three cut-off points; after the second, fourth, and sixth component. Based on Catell's (1966) Scree test, we decided to retain four components for further investigation. The four-component solution explained $49.3 \%$ of the total variance, with the component 1 through 4 explaining 19.3\%, 16.2\%, 7.1\%, and $6.7 \%$ of the total variance respectively. Based on the Scree test we assume that there are four variables underlying motivation and ability. To aid in the interpretation of these four components, Oblimin rotation was performed. The rotated solution revealed the presence of simple structure (Thurstone, 1947, as cited in Pallant, 2011), with all components showing several strong loadings. The different factors showed very weak intercorrelations (-.19, -.16, .03). To confirm the newly found structure of the variables, scale reliability was retested (see Table 5.1).

Table 5.1

Scale reliability after factor analysis.

\begin{tabular}{lcc}
\hline Contruct & \# of items & Cronbach's $\alpha$ \\
\hline Motivation & 11 & .85 \\
Personal motivation & 5 & .78 \\
External motivation & 6 & .77 \\
Ability & 17 & .81 \\
Personal ability & 5 & .60 \\
External ability & 12 & .86 \\
\hline
\end{tabular}


Following these findings, the variables social motivation and structural motivation, as well as the variables social ability and structural ability where combined, resulting in the new variables external motivation and external ability. Reliability analysis showed that the internal consistency of the personal ability scale was $\alpha=.60$. Because this was just within the latitude of acceptance, we decided to retain the personal ability scale as a research instrument.

\subsubsection{Relationships between research variables}

We used regression analyses to investigate the relationships between levels of motivation, ability, safety climate and safety performance. A regression analysis was conducted to determine the extent to which safety climate significantly predicted safety performance. Results confirmed H1, hypothesizing that safety climate is a predictor of safety performance levels (see Table 5.2 for a summary of the analysis). Safety climate also explained a significant proportion of variance $(60 \%)$ in levels of safety performance.

Multiple linear regression analyses were used to develop a model for predicting levels of safety climate based on levels of motivation, and ability (both for motivation and ability as a main construct and with sublevels of motivation and ability). Statistics and regression coefficients are depicted in Table 5.2. From the analysis it showed that only ability as a whole significantly $(p<.001)$ predicted safety climate, while the second analysis showed that only external ability significantly $(p<.001)$ predicted safety climate. The two predictor model (motivation and ability) was able to account for $54 \%$ of the variance in safety climate, while the four predictor model was able to account for $56 \%$ of the variance in safety climate.

Multiple linear regression analyses were used to develop a model for predicting levels of safety performance based on levels of safety climate, motivation, and ability (both for motivation and ability as a main construct and with sublevels of motivation and ability). Statistics and regression coefficients are depicted in Table 5.3. From the analysis it showed that all predictors significantly -safety climate $(p<.001)$, ability $(p=.001)$, and motivation $(p=$ $.022)$ - predicted safety performance, while the second analysis showed that only safety climate $(p<.001)$, personal motivation $(p=.004)$, and external ability $(p=.005)$ significantly predicted safety climate. The three predictor 


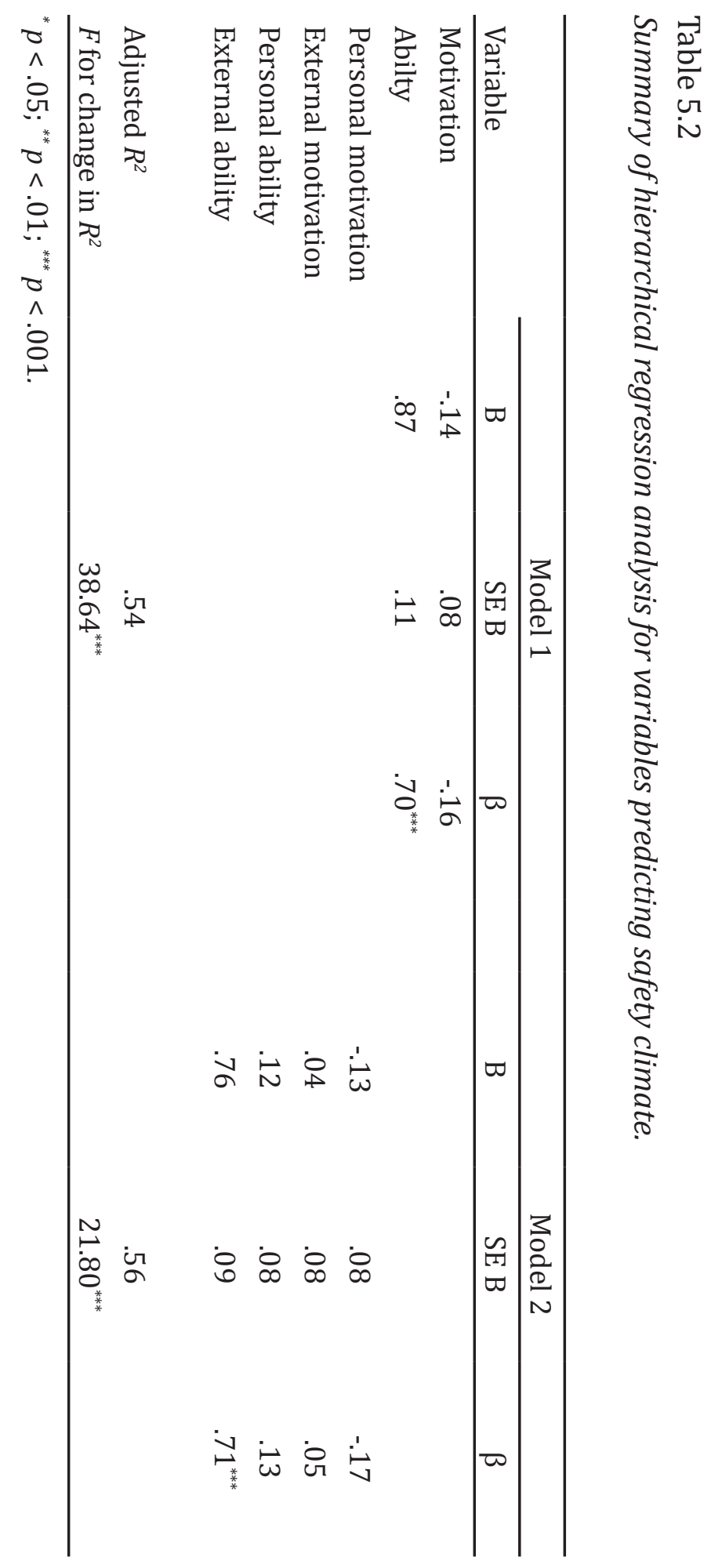




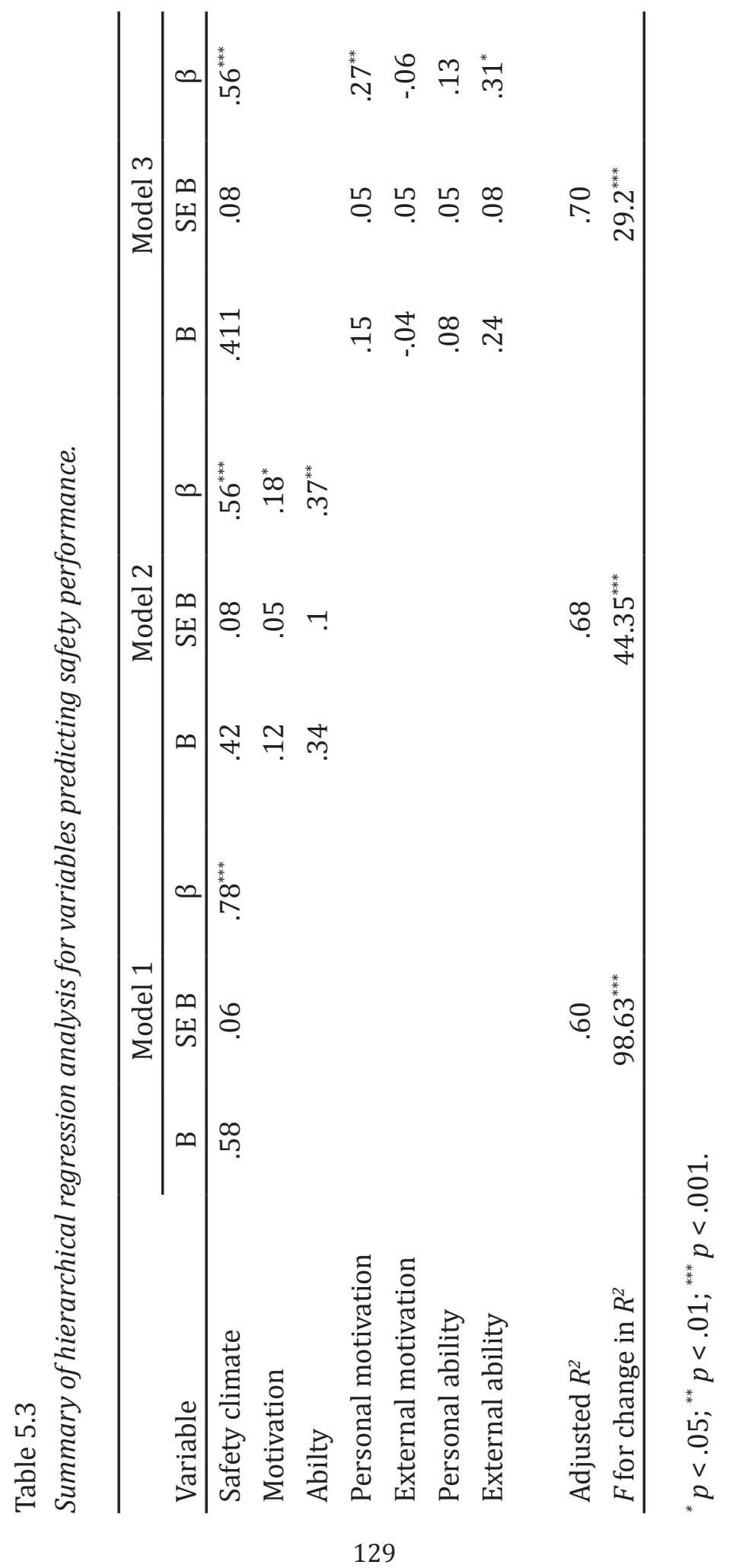


model (safety climate, motivation, and ability) was able to account for $68 \%$ of the variance in safety climate, while the five predictor model was able to account for $70 \%$ of the variance in safety climate.

Following the less well-established identification of the general importance of external ability for both safety climate and safety performance, we conducted a regression analysis for the separate items of those scales. This allowed us to see which elements needed to be highlighted in the design of safety instructions. The regression analysis showed that for safety climate, the strongest indicator was that 'Our management supplies enough safety equipment'. For safety performance, awareness that 'My co-workers use their personal protective equipment correctly' is the most important aspect. We will explore the implications of these findings in the discussion.

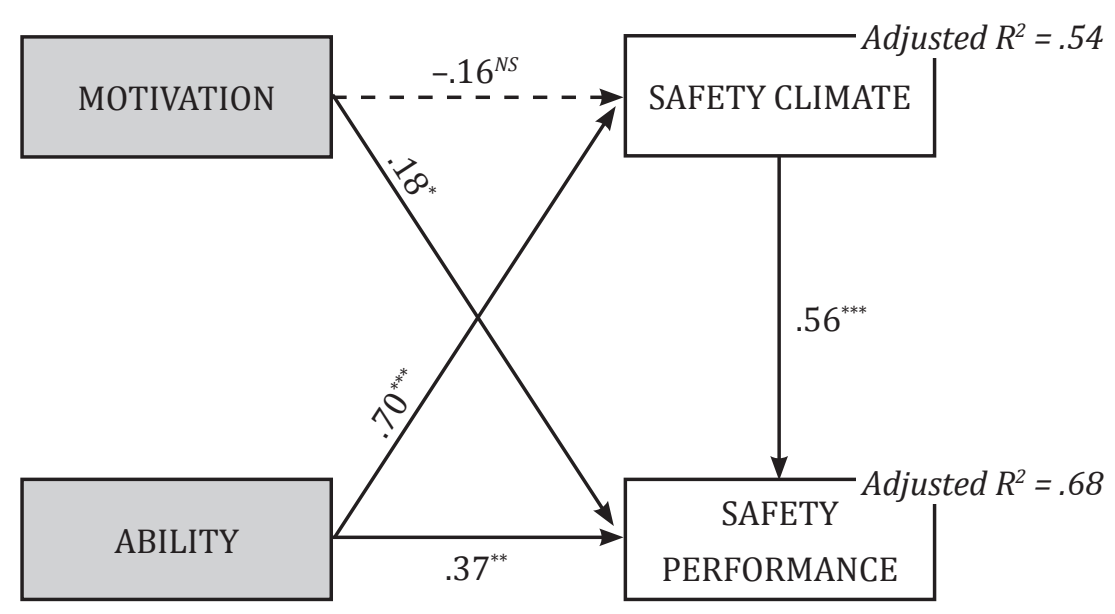

Figure 5.2. Regression model depicting the causal relation of motivation and ability on safety climate and safety performance.

Dashed lines indicate non-significance. ${ }^{*} p<.05 .{ }^{* *} p<.01 .{ }^{* * *} p<.001$. 


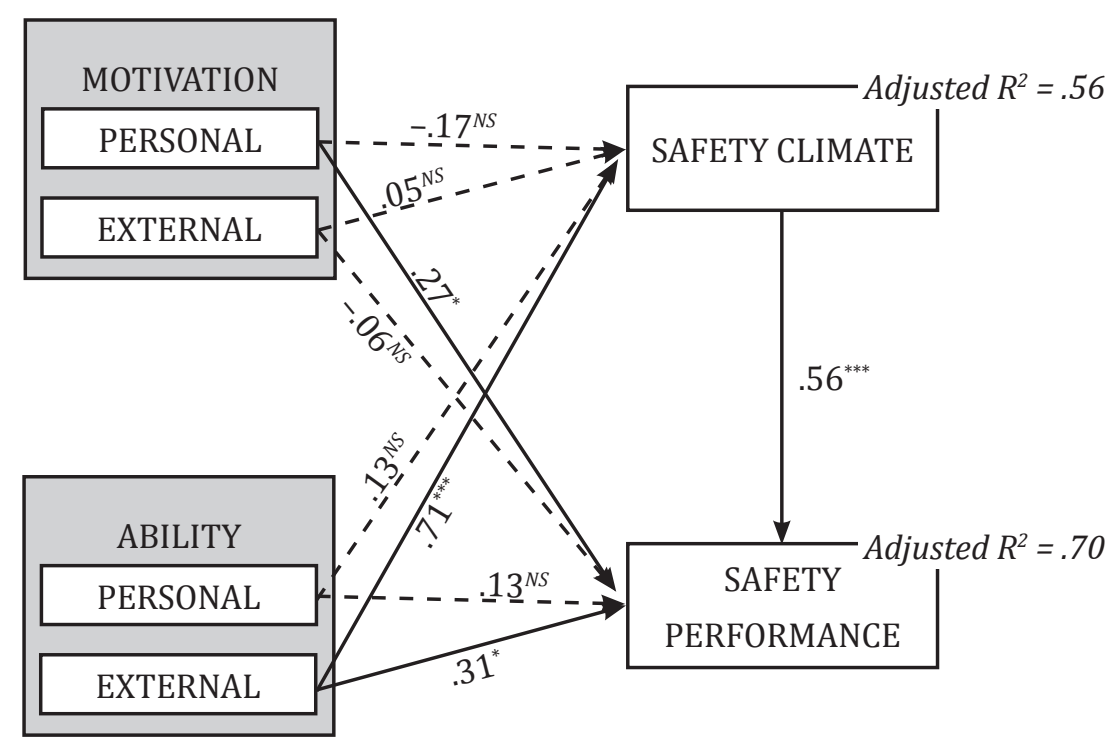

Figure 5.3. Regression model depicting the causal relation of the two levels of motivation and ability on safety climate and safety performance. Dashed lines indicate non-significance. ${ }^{*} p<.05 .{ }^{* *} p<.01 .{ }^{* * *} p<.001$.

\subsection{Discussion}

This study confirms the causal relation between safety climate and safety performance (H1) as hypothesised and shown in previous research (DeJoy et al., 2004; Guldenmund, 2000; Gyekye \& Salminen, 2009; Jiang et al., 2010; Neal et al., 2000; Neal \& Griffin, 2006; Pousette et al., 2008; Silva et al., 2004), and the finding that this relation is partially affected by knowledge (i.e., ability) and motivation (Zhou et al., 2008). Additionally, this study shows that the Safety Climate scale developed by Hahn and Murphy (2008), as well as the shortened version of the safety performance scale developed by Whu et al. (2008) can be seen as reliable, as they showed good internal consistency, which reconfirms their usability for future research.

This study shows the importance of two levels of motivation and ability as predictors of safety climate and safety performance. Making use of factorial 
analysis, we were able to distinguish between personal and external motivation, and between personal and external ability. The latter showed the strongest power in predicting safety climate and safety performance.

The research model was based on the assumption that higher (sub)levels of motivation and ability would result in higher levels of safety climate and safety performance. The results from the regression analysis confirm the predicted relations between motivation and safety performance (H3), between ability and safety climate (H4), and between ability and safety performance (H5). However, the model was able to predict a greater sum of the variance in safety climate (56\%) and safety performance (70\%) with the inclusion of the different sublevels, compared to only motivation and ability ( $54 \%$ for safety climate, and $68 \%$ for safety performance). This indicates that the inclusion of different levels, as done by Hofmann et al. (1995), is justified.

For motivation, only the sub construct of personal motivation proved to be a significant predictor for safety performance, thereby confirming hypothesis H3a. Of both the ability sub constructs, only external ability proved to be a significant predictor, for both safety climate and for safety performance after controlling for safety climate. Thus, hypothesis $\mathrm{H} 4 \mathrm{~b}$ and $\mathrm{H} 5 \mathrm{~b}$ were confirmed. This indicates that higher levels of (external) ability predict higher levels of safety climate and safety performance.

Although not statistically significant, the direction of motivation and its sublevels as predictors of safety climate differed from the hypothesis. Instead of a positive relation, the relation between (personal) motivation and safety climate was negative. This indicates that a person will feel less motivated to pursue safety if he or she perceives the environment as safe. A similar result was found for external motivation as a predictor of safety performance, which indicates that higher levels of external motivation result in lower levels of safety performance. This implies that an increase in effort to motivate an employee externally results in lower levels of safety performance as perceived by that employee. These findings are in line with previous research into risk behaviour (Brewer, Weinstein, Cuite, \& Herrington, 2004).

\subsubsection{Implications for safety instructions}

The findings of this research -that personal motivation and external ability 
are the largest predictors of safety climate and safety performance- have important implications for practitioners. If motivation and ability are not the singular constructs they are commonly thought to be, materials used to enhance or affect them should be changed accordingly.

According to Geller (2003, as cited in Saleh, 2011, p. 5), safety is internally derived, meaning that individuals must understand the why behind the 'rules' and 'procedures.' The necessity of personal motivation in safety instructions was also found in a study by Elling (as cited in Hale, 1990), who found that $71 \%$ of the employees thought too little motivation was given to follow the instructions. The inclusion of personal motivation also forces writers of safety instructions to think beyond the interests of the organisation (Elling, 1997), and to extend the focus beyond merely technical requirements (Herrero et al., 2002) and being unrealistic (Hale \& Borys, 2013), which is common criticism on contemporary safety instructions. Personal motivation could be included in safety instruction by raising the risk awareness (Harvey, Bolam, Gregory \& Erdos, 2001) of employees, for example through mentioning negative outcomes of non-compliance.

A noteworthy finding from this research is the fact that external ability plays an important role in affecting levels of safety climate and safety performance. The additional analysis into the relation between external ability on the one hand and safety climate and safety performance on the other showed that the top 5 items were related to the availability, training, and use of safety equipment. Although the importance of external ability is seemingly eliminating the need for ability of the individual, the fact that the ability of the co-workers and the organisation is of such great importance to the individual employee stresses the importance of proper training of every employee in the organisation (Burke et al. 2006; Dedobbeleer \& German, 1987). We propose that external ability in an organisation can serve as a form of behavioural modelling through which the individual employee can see how he or she should interpret safety instructions and put them into practice (Racicot \& Wogalter, 1995), as well as enhance the self-efficacy of employees through proxy efficacy (Bray, Gyurcsik, Culos-Reed, Dawson \& Martin, 2001). Proxy efficacy 'reflects one's confidence in the skills and abilities of a third party or parties to function effectively on one's behalf' (Bray et al., 2001, p. 426), but is also thought to work the other way around, contributing to successful behavioural adaptation (Bandura, 1997, as cited by Bray et al., 2001). One 
way in which external ability could be incorporated in safety instructions is by emphasizing that employees are and can be a role model to their colleagues. Such inclusion could (partly) account for the effect co-workers can have on individual behaviour (e.g., 'My co-workers use their personal protective equipment correctly'). An additional benefit here could be that personal motivation and external ability can strengthen each other through proxy efficacy.

The other important items resulting from the additional analysis place an emphasis on the management. We therefore propose expressing management commitment in safety instructions. However, one should be cautious here. Hale (1990, p. 4) states that: 'Imposed safety rules are often seen as in conflict with other imposed rules of a higher priority' (e.g., production). This notion, mirrored to the heroes described by Bergson (Dragga, 2011, p. 6), points out that if management sets safety as their priority but does not walk the talk, then the behaviour that is seen as heroic is that of production and not safety. This imposes serious threats to the safety of every single employee.

\subsubsection{Limitations and implications for research}

Notwithstanding the contribution of this research to the understanding of workplace safety, and its confirmation of previous findings, it has some limitations. Although the coverage among employees achieved in the organisation was high (around 90\%), the number of participants $(N=75)$ is up for improvement. We recommend to replicate this research in different settings and organisations to verify our findings. A second limitation comes from barriers that exists in 'capturing' behaviour and emotions through the use of questionnaires. This limitation is twofold, as it refers both to the measurement of actual thoughts and emotions, and to the fact that the questionnaire was unable to 'capture' all of the hypothesised sublevels of motivation and ability. The latter may have occurred because such a perceived difference between the levels was absent, or because the items in the combined scale cancelled each other out. The latter would explain why there is no statistically significant finding for social motivation; a force that has been shown to be of influence numerous times in previous research (e.g., Ajzen, 1991; Andriessen, 1978; Milgram, 1963). While some research has indicated that external motivation in the form of rewards and penalties is effective (e.g., Hasan \& Jha, 2013), there are strict limitations and also a large number of 
opponents (e.g., Schwartz \& Sharpe, 2010). Future research should therefore further investigate this gap between the proposed sublevels and the findings in this research.

\subsection{Conclusion}

This research studied the presence of sublevels of motivation and ability, and their relation with safety climate and safety performance. The findings indicate that including the sub constructs personal motivation and external ability is more valuable than measuring motivation and ability as a whole. Our findings indicate that in the design of safety instructions, focusing on personal motivation and external ability seems a promising strategy for contributing to a healthy and safe work environment. 


\section{Anv}
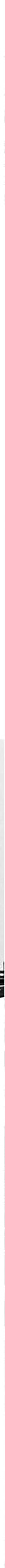

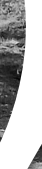

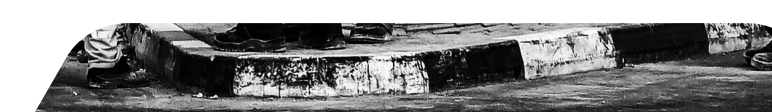


Chapter 6

\title{
Towards a broader understanding of safety communication: Development and initial validation of a measurement instrument
}

\author{
'To effectively communicate, we must realize that we are \\ all different in the way we perceive the world and use \\ this understanding as a guide to our communication with \\ others.' \\ Tony Robbins
}

Cornelissen, P.A., Van Hoof, J.J., \& De Jong, M.D.T. 


\subsection{Introduction}

Communication has been a long-recognised element in studies regarding occupational safety where it is considered of importance for the achievement of organisational health and safety objectives (Vassie \& Lucas, 2001). Communication is generally considered to minimise safety outcomes such as incidents, accidents and injuries (e.g., Hofmann \& Morgeson, 1999) and increase safety performance (Cornelissen et al., 2017). Yet despite its importance and positive influence on occupational safety, the concept of safety communication lacks a clear and comprehensive understanding.

First, within research there is no clear and commonly agreed upon definition of safety communication. Safety communication definitions range from anything as abstract as 'the open, free-flowing exchange about safety-related issues' (Siu, Philips, \& Leung, 2004) to something as narrow as 'giving feedback via performance statistics' (Vecchio-Sadus, 2007). In contrast to the variation in safety communication definitions, existing safety communication measures seem to focus on the communication between supervisors and subordinate (e.g., Cigularov, Chen, \& Rosecrance, 2010; DeJoy et al., 2004; Håvold \& Nesset, 2009; Hofmann \& Stetzer, 1998; Michael et al., 2006; Vandenberg, Richardson, \& Eastman, 1999; Zohar, 2002). The origins of many of these measurement scales can be traced back to previous scientific work. For example, Ciguralov's et al. (2010) 5-item safety communication scale was based on the 7-item scale by Hofmann and Morgeson (1999). In turn, the Hofmann and Morgeson scale is identical to the scale developed by Hofmann and Stetzer (1998). While supervisor-subordinate communication might be an important aspect of safety communication, such a unilateral focus is at odds with the variety in definitions and with the wide range of different aspects that are considered to be of importance for successful communication (e.g., aspects of the message; implicit communication). Current scales might therefore capture just part of the safety communication construct.

Second, there is a lack of agreement among researchers on how safety communication fits with other safety concepts. Researchers have conceptualised safety communication as part of safety climate (e.g., Cheyne et al., 1998; Neal et al., 2000) or safety culture (e.g. Behm \& Schneller, 2013; Boughaba et al., 2014), or conceptualised safety climate as an antecedent 
of safety communication (e.g., Hofmann \& Stetzer, 1998). Related to this ambiguity regarding the safety communication concept are the different expectations concerning the relationship between safety communication and occupational safety. While some researchers suggest safety communication to affect occupational safety through safety climate (e.g., Clarke, 2006) or safety culture (e.g., Choudhry, Fang, \& Mohamed, 2007), others think this influence of safety communication on occupational safety to be direct (e.g., Alsamadani et al., 2013a).

Given its perceived importance and potential for occupational safety, a more comprehensive understanding of safety communication is much needed. This need is further underlined by the notion that organisational and managerial communication are often discussed in line with a viewpoint that assumes communication to be easy and to require 'very little effort to be done successfully' (Axley, 1984, p. 434). Such a view on communication can have negative consequences, especially for organisations in which great numbers of lives and material resources can be affected by miscommunication (Axley, 1984).

The aim in this study is twofold. First, through the development of a quantitative measure of safety communication we aim to contribute to a more comprehensive understanding of the safety communication concept. In addition to an increased understanding, the development of a quantitative measure provides practitioners with a practical instrument that can be used to assess the perceived quality of safety communication within organisations, identify potential points for improvement in current (safety) communication efforts and can be used to assess the effectivity of interventions and programs. The second aim in this study is to assess the relationship of safety communication with safety climate and two other, widely used antecedents of safety performance: safety knowledge and safety motivation (Christian et al., 2009).

\subsection{Theoretical framework}

Below, an overview is provided of the different additional aspects of safety communication identified in literature which are thought to be important for successful safety communication. First, the different directions in which 
communication can take place and the corresponding preconditions for success are discussed. Second, aspects thought to be facilitating for reaching the target audience are discussed. Lastly, influential aspects of the sender are discussed.

\subsubsection{Directions of communication}

As reflected in the measurement scales, safety-related communication is generally associated with downward, one-way communication between supervisors and subordinates (Michael et al., 2006). For such communication to become effective, frequent interactions are deemed vital (e.g., Alsamadani et al., 2013a; Cigularov et al., 2010; Fernández-Muñiz, Montes-Peón, \& VázquezOrdás, 2012; Ghosh, 2014). In addition to communication between people on different hierarchical levels (i.e. vertical communication), communication can also occur between employees operating on the same hierarchical level, which is referred to as horizontal communication (Bartels, Peters, De Jong, Pruyn, \& Van der Molen, 2010). Two-way and open communication is believed to be more beneficial for occupational safety than one-way topdown communication (e.g., Ghosh, 2014; Michael et al., 2016; Vinodkumar \& Bhasi, 2010). Here, 'open' communication refers to the (perceived) freedom to communicate about safety and is considered important as it makes employees feel less nervous about raising and discussing safety-related issues with superiors (Cigularov et al., 2010; Törner \& Pousette, 2009). Although a dual view on the directionality of communication (i.e. vertical versus horizontal communication) is limited in the sense that it does not take into account mixtures of various directions that occur in the workplace (e.g., group meetings with supervisors and colleagues), it is a widely accepted and practically useful classification.

Related to the both the direction and perceived openness of communication is the concept of feedback. While the term is frequently used in the occupationalsafety context, it can serve several (partial-overlapping) functions such as correction, reinforcement, forensic diagnosis, benchmarking, and longitudinal development (Price, Handley, Millar, \& O’Donovan, 2010). While some researchers found feedback to be effective when used top-down as a mean for correction or benchmarking (e.g., Cooper, Philips, Sutherland, \& Makin, 1994; Fellner \& Sulzer-Azaroff, 1984), others found either mixed results (Lee, Shon, \& Oah, 2014), or didn't find any effect (Pettersson-Strömbäck, Liljelind, Neely, 
\& Järvholm, 2008). Safety-related feedback can also be present in the form of employees reporting unsafe situations or conditions to their superiors or colleagues.

\subsubsection{Reaching the target audience}

For communication to become effective, the target audience has to be reached (Nöhammer, Schusterschitz, \& Stummer, 2010). Within organisations, this can be accomplished through both formal and informal modes of communication (Alsamadani et al., 2013a; Alsamadani, Hallowell, JavernickWill, \& Cabello, 2013b), both of which have been demonstrated to be important (e.g., Alsamadani et al., 2013a, Cavazotte, Duarte, \& Gobbo, 2013; Zohar, 2002). Whereas informal safety communication refers to unscheduled communication (e.g., ad hoc conversations during planning or in the workplace), formal safety communication refers to scheduled forms of communication, such as toolbox meetings, posters and communications in written form (Alsamadani et al., 2013b).

Unfortunately, previous research on job communication (Clarke, 2006) and health and safety objectives (Vassie \& Lucas, 2001) has illustrated that reaching everyone is not an objective that always comes natural in organisations. However, organisations might improve their reach by making their employees more receptive to occupational health programs through the use of personal notifications (Stummer, Nöhammer, Schaffenrath-Resi, \& Eitzinger, 2008, as cited in Nöhammer, et al., 2010), attractive and motivating designs, as well as leaving employees the autonomy to decide to what extent they want to participate in occupational health programs (Nöhammer et al., 2010).

\subsubsection{Sender characteristics and behaviour}

While these elements already illustrate that safety-related communication includes a greater variety of topics than just supervisor-subordinate communication, they might leave the (unwarranted) impression that communication is a deliberate process. However, communication can also occur through the unintentional transmission of stimuli (Nilsen, 1957). Such communication -for example in the shape of exemplary behaviours- should also be taken into account as the unintentional transmission of stimuli 
might be as, or even more important for occupational safety than intentional transmission of words (Van Dyck, Dimitrova, De Korne, \& Hiddema, 2013).

Related to the unintentional transmission of stimuli are employees evaluations of the sender's credibility and commitment. Employees cognitively evaluate the information communicated to them by their supervisors (Luria, 2010). Their response towards communication about risks and the acceptance of such communication is influenced by their evaluation of the credibility of the sender, rather than the actual content of the information (Conchie \& Burns, 2009; Frewer \& Miles, 2003). Employees that trust their supervisor 'are more likely to reach similar conclusions about the importance of safety' (Luria, 2010, p. 1293).

The importance of the sender is further stressed by the notion that while communication is considered a fundamental tool for increasing employee involvement, it is conditioned by (perceived) management commitment (Fernández-Muñiz et al., 2012). The importance of management commitment for occupational safety has been underlined in numerous studies (e.g., Abudayyeh, Fredericks, Butt, \& Shaar, 2006; Vredenburgh, 2002; Zohar, 1980).

\subsubsection{In sum}

The literature discussed above illustrates that safety communication is much broader than mere supervisor-subordinate communication, and the need to incorporate -among others- the different directions in which communication can take place, frequency of communication, perceived openness, feedback, aspects related to the reach and design of communication, unintentional and intentional forms of communication, and aspects related to the sender into a measure of safety communication. In order to come to a more comprehensive overview, the topics identified in literature will be further complemented through expert rounds.

\subsection{Method}

In order to complement and refine the topics discussed in the theoretical framework, several separate expert rounds were conducted. Together, these formed the input for the further development of the measurement instrument. 


\subsubsection{Measurement development}

A first expert meeting was organised with five communication experts (academics). They were specifically targeted in order to gain a broader perspective on (safety) communication. By means of a brainwriting method the experts were asked to list topics they thought to be important for, or related to safety communication. Brainwriting is similar to brainstorming in the sense that people come together to generate (new) ideas, but instead of vocally sharing ideas participants are asked to write down their ideas on sheets of paper. These sheets are then circulated among participants, who can build on each other's ideas and add their own (new) ideas to the sheets of paper. There is some evidence that this method outperforms traditional brainstorming groups in terms of idea-generation (Heslin, 2009). After the meeting the input of the communication experts was summarised and compared with the 12 topics from the theoretical framework, resulting in the identification of 14 additional topics, and one additional dependent variable that should assess employee evaluations of the organisation's own safety communication in comparison with that of other organisations.

Based on the combined input from the theoretical framework and the brainwriting session two of the authors generated five items per topic, creating an initial pool of 130 items. This initial item pool was then sent to the scientific experts that participated in the brainwriting groups for them to give feedback on, leading to adjustments in several items and merger of 6 topics due to overlap (e.g., match with practice and relevance of communication). In order to measure the control variables (e.g., safety knowledge, safety motivation, safety climate) existing (validated) scales were used in order to facilitate an easier development process. Safety knowledge was assessed using the six-item scale developed by Vinodkumar and Bhasi (2010) and safety climate was assessed using the six-item scale developed by Hahn and Murphy (2008). Due to the (partial) overlap in the safety motivation scales developed by Neal and Griffin (2006) (3 items) and Vinodkumar and Bhasi (2010) (6 items), the items from both scales were included.

A second expert round was held with two experts from practice in the field of safety communication. They were asked to match the individual items to the different topics to validate the intended item-topic match. During this 
process, they were encouraged to make remarks about individual items.

Their feedback led to the merger of two topics and textual adjustments in 27 items. The resulting item pool consisted of a total of 110 items that covered 22 communication-related topics (see Table 6.1) and 24 items that covered the four dependent variables of safety knowledge, safety motivation, safety climate and safety communication comparison.

Table 6.1

Overview of topics identified through literature review and expert rounds after revision.

\begin{tabular}{ll}
\hline Source & Topics \\
\hline Identified in literature & - Vertical communication \\
& - Horizontal communication \\
& - Evaluations \& follow-up on feedback \\
- Possibilities for providing feedback & - Reach of communication \\
& - Formality of communication \\
& - Attractiveness of communication \\
& - Cxemplary behaviours \\
- Management commitment
\end{tabular}

Additional topics identified - Actuality of information through expert rounds

- Availability of information

- Balance between positive and negative attention for safety

- Communication channels

- Comprehensibility of information

- Match with practice

- Quantity of communication

- Structured communication

- Timing of communication

- Tone in communication

- Unambiguity in communication 
After the feedback from both the scientific and field experts was processed, the item pool was qualitatively assessed in terms of legibility and intelligibility. The item pool was presented to a group of 15 blue collar workers employed at an industrial services provider in the Netherlands. In order to minimise the cognitive load of the task and the impact of the task on their working hours, the participating employees were divided into three groups of five employees. Each of these groups was presented with one third of the total item pool. Participants were first asked to fill out the questions belonging to their part of the questionnaire. Upon completion of the questionnaire a small group discussion followed in which participants were encouraged to criticise the items. Remarks were carefully noted and resulted in small adjustments of three items and the addition of a 'not applicable to my work situation' answer option.

\subsubsection{Administration of the questionnaire to a development sample}

Organisations were recruited via two safety-themed groups on a social online network for professionals and via the personal network of one of the authors. Of the eleven organisations that responded, four agreed to participate in the research. All four organisations originated in the Netherlands and active in the fields of construction, industrial services or engineering. Within the participating organisations a combined total of around 1500 potential respondents were approached. A total of 340 questionnaires were returned (23\% response rate), of which 252 were retained after scanning the data. On average, participants' were 45 years old $(N=229)$, with a tenure of 12 years $(N=218)$. As not every participating organisation allowed asking the gender of participants, the distribution of participating males and females could not be presented here.

\subsubsection{Procedure}

In consultation with the participating organisations, questionnaires were distributed to employees via the internet using Qualtrics (www.qualtrics.com), in print, or a combination of the two. For each organisation the procedure was slightly different as not all potential participants were allowed to fill in the questionnaire during work-time. 
In all cases, participation was on a voluntary bases. Both the online version and the print version of the questionnaire started with a short description of the research's purpose and an informed consent form, followed by instructions on how to fill out the questionnaire. The questionnaire then continued with the safety communication-related statements, followed by the statements used for the control variables. The last part of the questionnaire contained several general questions regarding demographics and allowed participants to leave comments regarding the questionnaire or the organisation. Data from online completed questionnaires were downloaded while the printed questionnaires were gathered per organisation and then send to the researchers. The data were then digitalised and combined into one data file.

\subsubsection{Analysis}

The data were entered into SPSS (Statistical Package for the Social Sciences, version 25), and the file was checked for errors. As a result, the data of 88 respondents (26\%) were removed due to either non-completion or large parts of missing data, leaving a total of 252 respondents. Subsequently, the answers to negatively formulated statements were recoded and preliminary analyses were conducted to examine whether the data were suitable for analyses.

Subsequent data analyses were conducted in consecutive steps. First, the underlying structure of the safety communication items was analysed using principal component analysis (PCA). Second, reliability analyses were conducted to assess the internal reliability of the extracted factors as well as the measures for safety knowledge, safety motivation, safety climate and the items that asked participants to compare the organisation's safety communication efforts with other organisations. Third, building on the factors (i.e. components) extracted through PCA, bivariate and partial correlation analyses were conducted to explore the (inter)relationships between the communication factors and the dependent variables related to occupational safety.

\subsection{Results}

The data were first subjected to preliminary analyses. Then, principal component analysis (PCA), reliability analyses, bivariate and partial correlation analyses were conducted. 


\subsubsection{Preliminary analyses}

Preliminary analyses were conducted to test whether data were missing (completely) at random using Little's MCAR test and examination of the percentage of missing data per item. A significant outcome was found for Little's MCAR test $\left(\chi^{2}=17473,211, \mathrm{df}=16806, p<.001\right)$, and a closer examination of the data revealed that for nine items the percentage of missing data exceeded the commonly excepted threshold of 5\%. Although case-wise deletion would be the best solution in further analysis, this would lead to a unacceptable amount of data to be excluded (Field, 2009). The decision was therefore made to continue with the analyses using pair-wise deletion.

\subsubsection{Scale construction}

A PCA was conducted on the 110 safety communication items with orthogonal rotation (varimax). Varimax rotation was used as we expected that potential factors underlying safety communication would be related. The KaiserMeyer-Olkin measure verified the sampling adequacy for the analysis, KMO $=.885$, which classifies as 'great' (Hutcheson \& Sofroniou, 1999, as cited in Field, 2009). The diagonals of the anti-image correlation matrix were all greater then .50, which is above the acceptable limit (Field, 2009). Bartlett's test of sphericity $\chi^{2}(5995)=17050,861, p<.001$, indicated that correlations between items were sufficiently large for PCA. Lastly, the communalities were all above .56, further confirming that each item shared some common variance with other items and that the sample size was sufficiently large for principal component analysis (MacCallum, Widaman, Zhang, \& Hong, 1999). Considering these outcomes, it was deemed appropriate to continue with the analysis.

An initial analysis was run to obtain eigenvalues for each component in the data. 24 components had eigenvalues over Kaiser's criterion of 1 and combined these components explained a large percentage $(71.5 \%)$ of the variance. The scree plot was slightly ambiguous due to the high number of potential components and showed inflexions that would justify retaining 4, 7, 11 and 13 components. Taking into account the theoretical substantiation, the large sample size, the scree plot and Kaiser's criterion, the decision was made to retain 13 components, which together explained over two-thirds (68.9\%) of the variance after rotation. Following Stevens' (2002, as cited in Field, 2009) 
recommendations, a total of 56 items were eliminated because they failed to have a primary factor loading of .40 or above on a factor (8 items), loaded strongly ( $>.40$ ) on multiple factors (36 items), were the only item loading on a factor (4 items), or were removed based on the content of the item (8 items). In the final structure, one item loaded strongly on two factors (above .40) but was retained as the difference between the factor loadings was large than .20. Table 6.2 shows the resulting factors, corresponding items, factor loadings after rotation, percentage of variance accounted for by each factor, and reliability estimates (Cronbach's $\alpha$ ). As the thirteenth factor (labelled formality) did not meet the general accepted standard of Cronbach's $\alpha$.70, the factor was excluded from further analyses.

Table 6.2

Summary of principal component analysis results, indicating extracted factors, corresponding items, factor loadings after rotation for each item, percentage of variance accounted for by each factor, and reliability estimates (Cronbach's $\alpha$ ).

\begin{tabular}{|c|c|c|}
\hline M1 & I find the design of the communication about occupational safety pretty & .76 \\
\hline M2 & $\begin{array}{l}\text { The communication about occupational safety matches my own use of } \\
\text { language well }\end{array}$ & .74 \\
\hline M3 & I find the design of the communication about occupational safety good & .73 \\
\hline M4 & The information about occupational safety is consistent & .69 \\
\hline M5 & $\begin{array}{l}\text { The various messages about occupational safety match well with one } \\
\text { another }\end{array}$ & .66 \\
\hline M6 & I find the information about occupational safety to be clear & .63 \\
\hline M7 & The quality of the communication means used is good & .62 \\
\hline M8 & The right tone is used in the communication about occupational safety & .60 \\
\hline M9 & $\begin{array}{l}\text { I find the tone that is used to communicate about occupational safety } \\
\text { pleasant }\end{array}$ & .56 \\
\hline M10 & Some of the information about occupational safety is not up-to-date & .42 \\
\hline
\end{tabular}


Table 6.2 (continuation)

Factor 2: Vertical communication (7.2\%, Cronbach's $\alpha=.84$ )

VC1 Within our organisation it is better to stay silent about unsafe situations, else you will get in trouble

VC2 I'd rather not talk about unsafe situations and incidents because it will result in negative consequences

VC3 It is difficult to talk about occupational safety with my supervisor(s) I am dissatisfied with the way my supervisor(s) address occupational safety topics with me

If employees contribute something about occupational safety they do not act on it

VC6 Supervisors do not care about occupational safety

Factor 3: Sender credibility (6.1\%, Cronbach's $\alpha=.89$ )
SC1 I trust the sender of communication about occupational safety
SC2 The sender of communication about occupational safety has sufficient knowledge and experience
SC3 The sender of communication about occupational safety is trustworthy $\quad .70$
SC4 I find the sender of communication about occupational safety unreliable $\quad .66$
SC5 I find the sender of communication about occupational safety unprofessional

Factor 4: Reach (5.5\%, Cronbach's $\alpha=.81$ )

R1 Not everyone receives the information about occupational safety Sometimes I do not receive certain information about occupational safety

R3 There is too little communication about occupational safety

R4 I am insufficiently aware of where I can find which information

Factor 5: Exemplary behaviours (5.4\%, Cronbach's $\alpha=.91)$

EB1 Supervisors wear the correct PPE (Personal Protective Equipment) themselves

EB2 Supervisors follow safety regulations

EB3

In general, managers give a good example concerning occupational safety 
Table 6.2 (continuation)

Factor 6: Timing (5.0\%, Cronbach's $\alpha=.76)$

There is a good balance between the quantity of information about

TI1 occupational safety that I receive via my colleagues and that I hear via $\quad .70$ my supervisor(s)

TI2 I receive the information about occupational safety when I need it .66

TI3 Communication about occupational safety occurs at the right moments .64

TI4 I am brought up to speed about changes in the area of occupational safety

Factor 7: Match with practice (4.9\%, Cronbach's $\alpha=.78$ )

MP1 The information about occupational safety that I receive is relevant for my function

I receive information about occupational safety that is unnecessary for my job

The information about occupational safety matches with my work

MP3 activities

MP4 I receive information about occupational safety that is not useful to me

Factor 8: Tone (4.7\%, Cronbach's $\alpha=.75)$

T01 I find the communication about occupational safety boring

T02 Concerning occupational safety, the tone if often too negative

T03 I find that communication about occupational safety is done in a childish way

T04 I find the tone used in the communication about occupational safety patronising

Factor 9: Feedback (4.2\%, Cronbach's $\alpha=.81)$

FB1 Within our organisation it is common to provide feedback about occupational safety

FB2 Within our organisation it is easy to give feedback about occupational safety

FB3 Within our organisation it is normal to talk about unsafe situation and safety incidents 
Table 6.2 (continuation)

Factor 10: Dissemination failures (4.1\%, Cronbach's $\alpha=.74$ )

DF1 Sometimes the communication about safety does not happen according to plan

DF2 Sometimes I hear about safety-related matters too late

DF3 The information about occupational safety is not available at the times that I need it

Oftentimes I am not informed about the latest changes concerning

DF4 occupational safety within our organisation

Factor 11: Communication channels (3.9\%, Cronbach's $\alpha=.72$ )

CC1 Our organisation uses communication channels that I do not use to communicate about occupational safety

Our organisation uses to many different communication channels to

CC2 communicate about occupational safety

Factor 12: Horizontal communication (3.8\%, Cronbach's $\alpha=.71$ )

HC1 I can talk freely about occupational safety with my colleagues

HC2 It is difficult to talk about occupational safety with my colleagues

HC3 I am satisfied with the

Factor 13: Formality (2.8\%, Cronbach's $\alpha=.49)$

F01 I like that I receive information about occupational safety via my supervisor(s)

F02 I like it that I receive part of the information about occupational safety via direct colleagues 
Reliability analyses were conducted for the scales intended to measure safety knowledge, safety motivation and safety climate (see Table 6.3). Based on the reliability analysis, one item (i.e. 'I believe that safety can be compromised for increasing production') was removed from the safety motivation scale developed by Vinodkumar and Bhasi (2010). Due to the higher internal reliability of the safety motivation measure developed by Vinodkumar and Bhasi compared to the one developed by Neal and Griffin, the choice was made to continue with the first in subsequent analyses. Based on the reliability analysis, one item (i.e. 'There are no major shortcuts taken when worker health and safety are at stake') was removed from the safety climate scale developed by Hahn and Murphy (2008).

Table 6.3

Reliability estimates (Cronbach's $\alpha$ ) for dependent variables.

\begin{tabular}{llcc}
\hline & & \multicolumn{2}{c}{$\begin{array}{c}\text { Cronbach's } \alpha \\
\text { coefficient }\end{array}$} \\
\cline { 3 - 4 } Measure & Source & $\begin{array}{c}\text { Original } \\
\text { study }\end{array}$ & $\begin{array}{c}\text { Current } \\
\text { study }\end{array}$ \\
\hline Safety knowledge & Vinodkumar and Bhasi (2010) & .77 & .84 \\
Safety motivation & Vinodkumar and Bhasi (2010) & .77 & .81 \\
& Neal and Griffin (2006) & $.85-.92$ & .76 \\
Safety climate & Hahn and Murphy (2008) & $.71-.85$ & .77 \\
Safety comm. & & & .85 \\
comparison & - & - & \\
\hline
\end{tabular}

\subsubsection{Correlation analyses}

Preliminary analyses of the data revealed that regression analysis would not be appropriate as not all of the assumptions could be met. Therefore, correlation analyses were conducted using Spearman's rho $\left(r_{s}\right)$ correlation coefficient instead of Pearson's $r$.

First, bivariate correlation analyses were conducted to assess the strength of the (inter)relationships between the safety communication variables and safety knowledge, safety motivation, safety climate and safety communication comparison. All variables were significantly correlated with each other, with 
the strength of relationships ranging from small to large (Pallant, 2007). The means, standard deviations and correlation coefficients of the variables are depicted in Table 6.4.

Second, in line with the findings from literature and the resulting model from the meta-analysis by Christian et al. (2009), partial analyses were conducted to statistically control for the variance shared between the safety communication variables and the dependent variables. A first partial correlation analysis was used to explore the relationship between the safety communication variables and safety motivation, while controlling for the influence of safety climate (see Table 6.5). An inspection of the zero order correlation for safety motivation suggests that controlling for safety climate has a large negative effect on the strength of the relationships between the safety communication variables and safety motivation. Only the variable sender credibility correlated significantly $(p<.05)$ with safety motivation when safety climate was controlled for (cf. Table 6.4 with Table 6.5).

A second partial correlation analysis was used to explore the relationship between the safety communication variables and safety knowledge (see Table 6.5), while controlling for the influence of both safety climate and safety motivation. An inspection of the zero order correlation for safety knowledge suggests that although controlling for safety climate and safety motivation has a negative effect on the strength of the relationships between the safety communication variables and safety motivation, this effect is smaller than it was for safety motivation. After controlling for the variance shared with safety climate and safety motivation, there were still five communication variables (i.e. vertical communication; reach; tone; communication channels; and horizontal communication) that correlated significantly with safety knowledge (cf. Table 6.4 with Table 6.5).

A third partial correlation analysis was used to explore the relationship between the safety communication variables and safety communication comparison, while controlling for the influence of safety climate, safety motivation and safety knowledge (see Table 6.5). An inspection of the zero order correlation for safety communication comparison suggests that although controlling for safety climate, safety motivation and safety knowledge has a negative effect on the strength of the relationships between the safety communication variables and safety communication comparison, this effect 


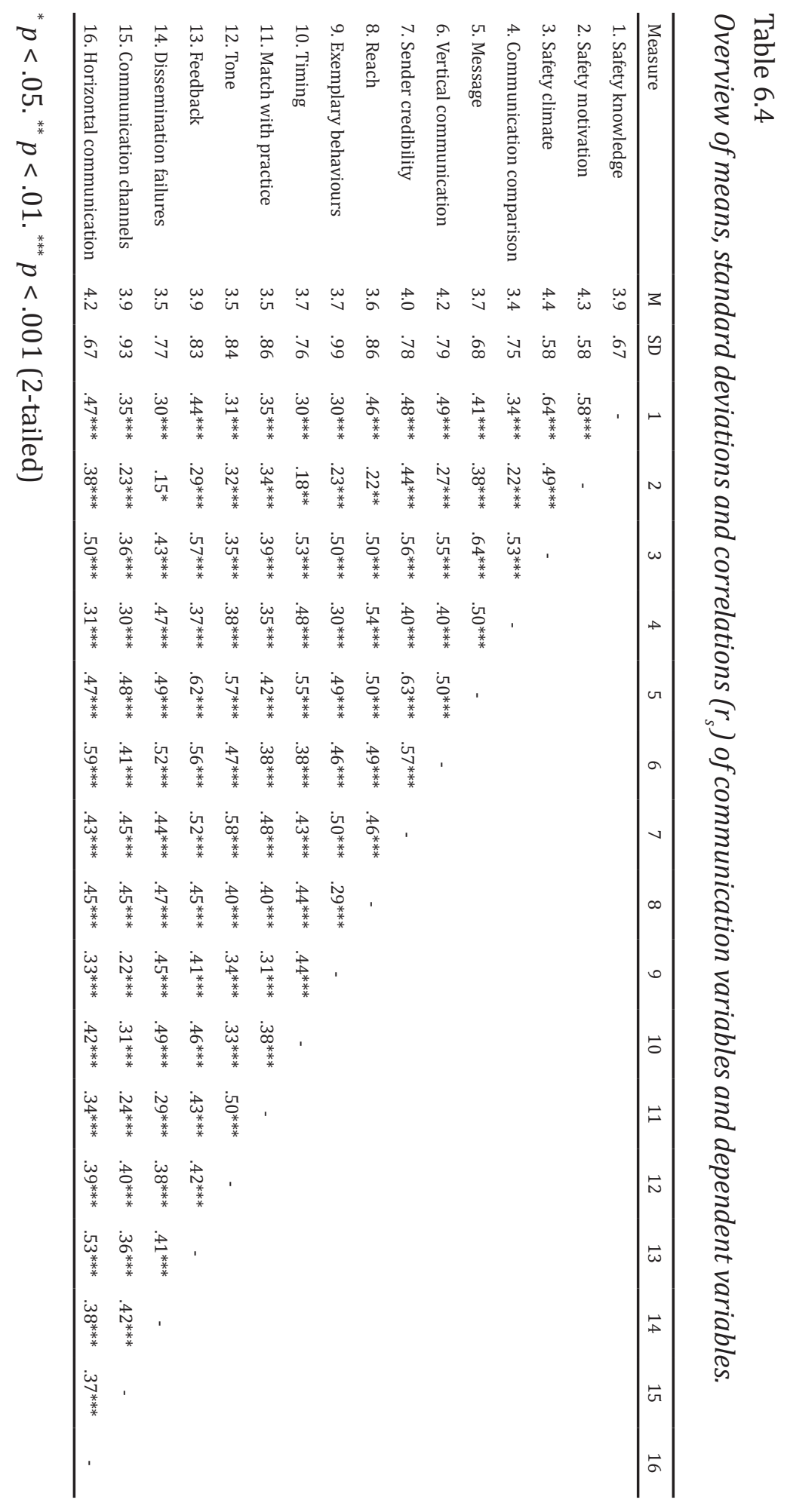




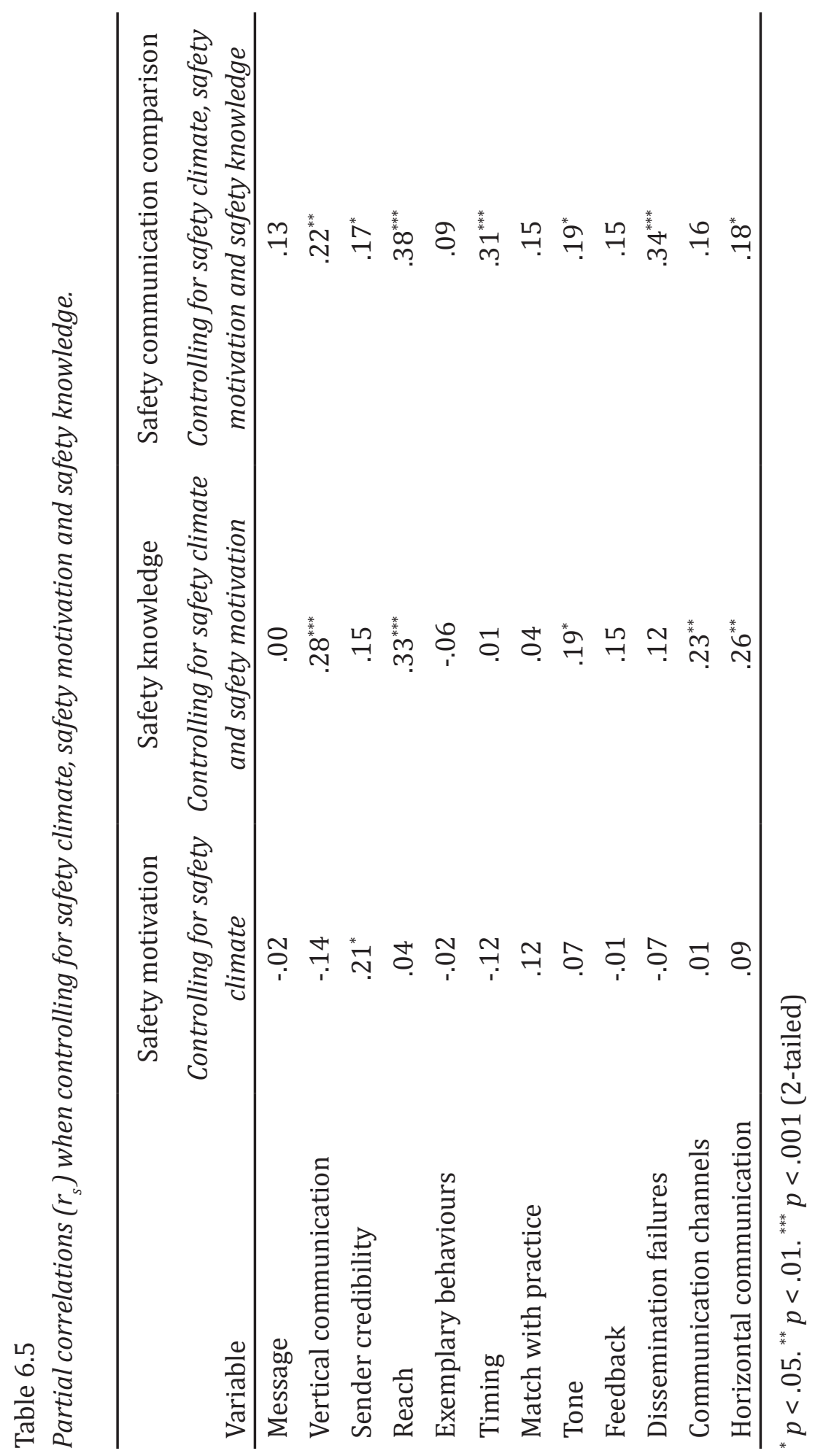


is smaller than it was for safety motivation and safety knowledge. Even after controlling for the variance shared with safety climate, safety motivation and safety knowledge, a majority of seven variables (i.e. vertical communication; sender credibility; reach; timing; tone; dissemination failures; and horizontal communication) correlated significantly with safety communication comparison (cf. Table 6.4 with Table 6.5).

\subsection{Discussion}

This study illustrated the process of developing a safety communication measurement instrument and subsequent exploration of the (inter) relationships between the different aspects of safety communication and safety knowledge, safety motivation and safety climate.

\subsubsection{Safety communication structure}

The literature discussed in the theoretical framework and the outcomes of the experts rounds revealed the rather limited view on safety communication in existing safety communication scales, and the subsequent need to extend current views on safety communication. Analysis of data gathered through a development sample revealed a factor solution consisting of 13 factors that together explained over two-thirds (68.9\%) of the variance.

The strongest factor was Message, which is reflective of employee evaluations of aspects of the content and design of safety-related communication. Related to Message are the factors Tone, which is reflective of the extent to which employees dislike the (tone in) communication about occupational, and Match with practice, which reflects the extent to which employees perceive the information about occupational safety to be relevant for their job. The second strongest factor was Vertical communication, reflecting the perceived openness and ease to which employees feel they can talk about occupational safety with their supervisor(s). Related factors are Horizontal communication, reflecting the extent to which employees are satisfied with their interactions with colleagues, and Feedback, reflecting the perceived commonness and ease within the organisation to provide feedback about occupational safety. The third strongest factor was Sender credibility, which is reflective of the extent to which people think the sender of safety-related communication to 
be credible. Related to Sender credibility is the factor Exemplary behaviours, reflecting the extent to which supervisors and managers set a good example when it comes to occupational safety. The fourth strongest factor was Reach, which reflects the extent to which people in the organisation have access to safety-related information. Related factors include the correct Timing of safety related communication, Dissemination failures, which reflects the extent to which safety-related communication is executed according to plan, and the factor Communication channels, which is reflective of the extent to which the organisation uses the correct channels to communicate about occupational safety. The last factor was Formality, which reflected the extent to which people were content with the formal and informal modes to communicate about safety within the organisation. However, this factor failed to show good internal reliability and was therefore excluded from subsequent analyses.

The factors from the principal component analysis show overlap with the topics discussed in the theoretical framework and those contributed by the experts (cf. Table 6.1 with Table 6.2), although they are not a one-to-one match. Especially the factors Message, Reach and Tone seem to be reflective of multiple topics, whereas topics such as Evaluations \& follow-up on feedback and Actuality of information appear not to be represented. Future research should further validate the factor structure found here, and examine the extent to which it is representative for an overall evaluation of safety communications within organisations.

\subsubsection{Relationships with other safety variables}

The safety communication factors that resulted from the PCA were further examined by exploring the strength of their relationships with safety knowledge, safety motivation, safety climate and safety communication comparison. The results of the bivariate correlation analyses illustrate that all safety communication variables significantly and positively correlate with each other, as well as with safety knowledge, safety motivation, safety climate and safety communication comparison.

The results of the partial correlation analyses demonstrated that controlling for the variance shared with safety climate negatively impacted the relationships between the safety communication variables and safety motivation, with only sender credibility correlating significantly with 
safety motivation. The importance of the credibility of the sender seems in line with findings from previous research on the importance of sender evaluations for the acceptance of risk communication (Conchie \& Burns, 2009; Frewer \& Miles 2003; Luria, 2010). The partial correlation analyses assessing the relationships between the safety communication variables and safety knowledge while controlling for safety climate and safety motivation demonstrated that the relationships were more robust. Five variables (i.e. vertical communication; reach; tone; communication channels; and horizontal communication) still correlated significantly with safety knowledge when safety climate and safety motivation were controlled for. While the importance of vertical communication, reach, and horizontal communication were addressed in previous research (e.g., Ciguralov et al., 2010; DeJoy et al., 2004; Ghosh, 2014; Hofmann \& Stetzer, 1998; Michael et al., 2006; Nöhammer et al., 2010; Vinodkumar \& Bhasi, 2010; Zohar, 2002) and are to be of expected importance for the dissemination of safety information, the importance of tone might be more unexpected. Looking at the corresponding items, the results seem to indicate that the tone in safety communication should be positive, and avoid addressing employees in a childish or patronising way.

In sum, these findings seem to confirm previous conceptualisations of safety communication as part of safety climate (e.g., Cheyne et al., 1998; Neal et al., 2000). Although the safety communication variables and safety climate share a fair amount of variance, the findings demonstrate the added value of a separate evaluation of the various safety communication variables compared to a more general safety climate measure, especially since communication is one of the more practical points that organisations can address in create safer workplaces. The developed safety communication measurement instrument provides practitioners with a practical instrument to assess the perceptions of employees regarding the safety-related communication efforts of their organisation, and can be used to identify points of improvement in contemporary safety communication approaches.

\subsubsection{Limitations}

Although the current study has contributed to current understandings of safety communication, there are several limitations that need to be addressed. First, a large part of the data had to be removed due to either non-completion or a large percentage of missing data. Based on the place of the missing 
data (latter part of the questionnaire) and comments left by participants in the comment box, the high rate of non-completion can be attributed to a combination of the length of questionnaire and the similarity between items. Second, as several assumptions regarding normality and missing data could not be met, the generalisability of these results has its limitations. However, considering that employees from four different organisations participated, the results were rather unambiguous. Third, the data used in this study was collected among employees in Dutch organisations, limiting the generalisability of the findings to organisations in or from (culturally) different countries.

\subsubsection{Future research}

As two components extracted through the principal component analysis consisted of only two items (i.e. communication channels and formality), future research should further operationalise these factors to meet statistical standards. For formality we recommend splitting the component into two variables; informal communication and formal communication. In line with the descriptions by Alsamadani et al. (2013b) these two variables should more clearly reflect unscheduled and scheduled forms of communication.

In order to allow researchers to use, validate and further develop the safety communication measurement instrument developed in this study the original items are provided in the appendix. In addition to the original (Dutch) items, we also provide an English version of the items to allow for use and validation outside of the Netherlands. The Dutch items were translated using forward-backward translation with two native English translators that are fluent in Dutch language. Their individual forward translations were combined and compared, followed by a backward translation by a third translator. This backward translation was then compared to the original Dutch version, resulting in 10 inconsistencies (7.5\%), of which two were attributed to mistakes in the backward translation, three inconsistencies could not be translated to English in another way, and five inconsistencies led to adjustments in the English version of the questionnaire. Both the original Dutch and translated English items are provided at the end of this dissertation (see Appendix A). 


\subsection{Conclusion}

Through the development of a quantitative measure of safety communication this article contributed to a more comprehensive understanding of the safety communication concept. The results provide researchers with new insights concerning the underlying structure of safety communication and its relations with safety climate, safety motivation and safety knowledge. The final 54item instrument provides practitioners with a practical instrument to assess employee perceptions of safety communication within their organisation and identify potential points for improvement in current (safety) communication efforts.

\section{Acknowledgements}

We would like to thank Fred Aarsen, Susanne Barth, Jordy Gosselt, Joyce Karreman, Silvie Pothof, Marieke Rietveld and Mark van Vuuren for their constructive feedback and help in the development of this measurement instrument. This research did not receive any specific grant from funding agencies in the public, commercial, or not-for-profit sectors. 


Chapter 7

\section{General discussion}




\subsection{General discussion}

Confronted with the sheer number of (fatal) accidents and the immense impact of occupational incidents, accidents and injuries, the aim in this dissertation was to increase current scientific understandings of occupational safety and ultimately help practitioners to create safer work environments. Considering the different approaches and the wide variety of studied factors the first objective was to create a thorough overview of the current status of occupational safety research. Therefore, this dissertation started with a systematic review of occupational safety literature in order to answer the question:

\section{RQ 1: What is the current status of occupational safety research with regard to determinants of safety outcomes and performance?}

Considering the importance of communication for safe work environments, the following overall research question was formulated:

\section{RQ 2: What is the role of communication in occupational safety?}

\subsection{Main findings}

Chapter 2 describes a systematic review of the literature on occupational safety research between 1980 and 2016 conducted in the construction, warehouse, manufacturing, and (offshore) petrochemical sectors. In order to provide insights in the current status of occupational safety research, studied variables and their underlying relationships were extracted and grouped. The resulting overview provides several insights. First, the majority of occupational safety research has focussed on studying the influence of a wide variety of factors (e.g., factors related to workplace characteristics \& circumstances, climate \& culture, and employee characteristics) in relation to the outcome variables negative safety outcomes (i.e., incidents, accidents, and injuries) and performance (i.e., organisational and safety-related performance). Second, in studying negative safety outcomes and performance, 
most research has focused on factors related to management \& colleagues, followed by work(place) characteristics \& circumstances, and employee demographics. Lastly, the majority of studied relationships between the various independent factors and negative safety outcomes and performance lacked unequivocal outcomes. Consensus (set at a two-thirds majority) was only found for less than 7 percent of the variables related to negative safety outcomes and less than 11 percent of the variables related to performance, while the remaining relationships are either subject to debate or appear to be understudied. From the different dependent variable clusters, the cluster of management \& colleagues emerged as an interesting avenue for further research. Not only does it comprise variables that can be influenced by people within organisations, it also comprises variables that are used by managers and supervisors to manage occupational safety within organisations. In order to explore the role of occupational safety in supervisors' work, and their attitudes and perceptions regarding various safety management instruments a qualitative study was conducted.

Chapter 3 describes a qualitative study of supervisors' perceptions, attitudes, and beliefs towards (the management of) occupational safety. Hereby it expands on the findings from Chapter 2, and particularly on the findings in the management \& colleagues cluster. The outcomes of the semistructured interviews illustrate the complex role that occupational safety plays in supervisors' work. First, despite the important and substantial role of occupational safety within their work, supervisors have to balance occupational safety with other organisational and managerial objectives. Second, the results bring differences between both managerial levels and individual supervisors to light with regard to their safety beliefs. While most supervisors' believed the motivation for safety within the organisation to be intrinsic and sincere, others doubted this. Almost all supervisors agreed that every individual has a share in the responsibility for occupational safety within the organisation, although half of the supervisors distinguished between an overall responsibility and a final responsibility that they attributed to those higher in the hierarchy of the organisation. Concerning occupational accidents, none of the supervisors believed that all accidents could be prevented, and additionally they thought accidents to be mostly caused by unconscious actions. Furthermore, the majority of supervisors did not think all incidents were reported. Lastly, the results illustrate that supervisors' attitudes influence their willingness to use and the perceived efficacy of safety 
management instruments. From the various safety management instruments, communication emerges as an important element for both occupational safety and other safety management instruments. Given the finding that supervisors differ in their beliefs regarding the motivation for occupational safety within the organisation and their beliefs that most accidents occurred due to unconscious actions, more research is needed. In order to better understand the place of safety in supervisors' work, and explore the different rationales for safety-related thinking, decisions and behaviours, a second qualitative study was conducted, applying a new perspective to the interview data that discussed potential conflicting demands between safety and other organisational demands.

Chapter 4 describes the application of an institutional logics perspective to qualitative data gathered among managers. It aims to uncover, illustrate, and explain different rationales underlying safety-related thinking, decisions and behaviours. The results illustrate that an institutional logics perspective can be effectively applied to occupational safety. First, applying an institutional logics perspective uncovered the logics of the market, profession, and corporation in supervisors' safety-related thinking, decisions, and behaviours. Second, the institutional logics perspective also revealed examples of logic multiplicity between the logics of the market, profession, and corporation, uncovering the underlying causes of safety-related conflicts. Additionally, different solutions from managers in response to the encountered complexity emerged. The demonstrated applicability of the institutional logics framework to occupational safety provides researchers and practitioners with a (new) discourse that allows them to better understand seemingly irrational or unconscious safety-related thinking, decisions and behaviours. While these empirical studies both contributed to current understandings of the (perceived) importance and value of occupational safety for managers and supervisors, managers and supervisors are not the ones at risk in a typical organisation. In order to better enable supervisors and managers to manage occupational safety and increase the safety of employees, a better understanding is needed of how employees can be reached, motivated and enabled to work safely.

Chapter $\mathbf{5}$ describes a quantitative study of different levels of ability and motivation and their relationship with safety climate and safety performance. 
While the importance of safety motivation and safety knowledge for occupational safety has been long recognised, they have always been conceptualised as unidimensional concepts. A questionnaire study was conducted among employees of a logistics warehouse, measuring their motivation and ability on three different levels (i.e. personal, social, and external), and their scores on safety climate and safety performance. First, the results confirmed the relationship between safety climate and safety performance. Second, the results demonstrated that a two-dimensional (i.e. personal level and external level) understanding of motivation and ability increased the predictive value of these variables. Third, of the explored sub levels of motivation and ability, personal motivation and external ability proved most relevant for creating a good safety climate and good safety performance. These result stress the importance of motivational elements and management commitment in (the design of) safety instructions. In line with the importance of communication for occupational safety as indicated in Chapter 2, Chapter 3, and Chapter 4, the results illustrate that a broader view on, and better understanding of communication and its underlying elements may help improve levels of occupational safety.

Chapter 6 describes the development of a safety communication measurement instrument. While the results from the preceding chapters illustrated the (perceived) importance and effectiveness of communication for occupational safety, current measurement scales of safety communication hold a rather narrow and unilateral view on communication. In order to advance contemporary understandings of safety communication, a comprehensive safety communication measurement instrument was developed. Based on the combined input from literature, existing measures and qualitative input from experts, 22 safety communication-related topics were identified. These topics served as a starting point for the creation of an initial item pool. After pre-testing the initial item pool among blue collar workers $(N=$ $15)$, the items were provided to a development sample $(N=252)$ from four different organisations in the form of a questionnaire. Principal component analysis revealed a 13 factor structure underlying safety communication, of which 12 factors showed good internal consistency. These factors were reflective of employee evaluations of the message, vertical communication, sender credibility, reach, exemplary behaviour, timing, match with practice, tone, feedback, dissemination failures, communication channels and horizontal communication. The subsequent bivariate and partial correlation analyses 
revealed that the safety communication variables shared fair amounts of variance with safety climate, which is line with previous conceptualisations of safety communication as part of safety climate. However, even when the variance shared with safety climate was controlled for, sender credibility correlated significantly with safety motivation. Furthermore, after controlling for the variance shared with both safety climate and safety motivation, vertical communication, reach, tone, communication channels and horizontal communication still significantly correlated with safety knowledge. In sum, the developed instrument adds to a more comprehensive understandings of safety communication and provides researchers and practitioners with a practical instrument to assess employee perceptions of safety communication within organisations.

\subsection{Theoretical implications}

Based on the outcomes of the studies described in the individual chapters, several overarching conclusions can be made regarding safety in the work context and the role of communication.

First, occupational safety is subject to the dynamics of the organisation and influences from field the organisation operates in. Aside from a wide variety of internal and external factors that can influence safety performance and safety outcomes (Chapter 2), occupational safety is just one of the many objectives for managers, supervisors and even employees within organisations. As such it has to compete with other work objectives (e.g., production) for time and resources (Chapter 3), and is subject to the organisational priority for occupational safety at a given time. Additionally, employees' safety-related thinking, decisions and behaviour are continuously influenced by actors from both within and outside of the organisation. Confronted with multiple and divergent prescriptions for action coming from the professional, corporation and market logic, employees continuously have to balance the varying interests with occupational safety (Chapter 4). These outcomes give rise to concern regarding the (perceived) importance and place of occupational safety within organisations. The (perceived) priority of occupational safety might be influenced by ambiguity on who is responsible for occupational safety within the organisation and beliefs regarding the preventability of accidents (Chapter 3). Such perceptions and beliefs might adversely affect 
employees' perceived need to act upon safety issues. In addition to balancing occupational safety with other managerial objectives, some supervisors also indicated that they doubted whether the motivation of the organisation for occupational safety was intrinsic (Chapter 3). This notion is strengthened by the presence of the market logic in safety conflicts (Chapter 4), and findings by previous researchers who found strategic and financial considerations influential of approaches and actions towards occupational safety (e.g., O'Neill et al., 2016; Podgórski, 2000; Swuste et al., 2014). These findings create the need for future research to continuously monitor the position and (perceived) importance of occupational safety throughout organisations. Additionally, in studying occupational safety, future researchers should take the influence of the dynamic organisational environment into account.

Second, occupational safety is a very complex and broad issue. The general introduction (Chapter 1) and the outcomes of the systematic review (Chapter 2 ) illustrated the limited success with which researcher and practitioners have used a wide variety of approaches and variables to improve occupational safety. In addition to the wide variety of different possibilities and approaches the results from the systematic review (Chapter 2) also illustrate the extensive levels of ambiguity found concerning the effectivity for the vast majority of studied factors. Considering the uncertainty that exists regarding the effectivity of both approaches and specific factors, it is understandable that both academics and practitioners struggle with improving workplace safety. This even gets further complicated by the different levels at which important factors such as motivation and ability might work and should be addressed (Chapter 5). However, even when the effectivity of a specific factor has been demonstrated in numerous scientific studies, the dynamics of the organisation and personal perceptions, beliefs and attitudes might hinder efforts to improve occupational safety (Chapter 3 ). Organisational members might lack the time or resources to do justice to the safety objective as it has to compete with other organisational objectives (Chapter 3). Furthermore, employee beliefs regarding the preventability of and reasons for safety incidents may negatively influence the perceived necessity to act (Chapter 3). Moreover, the different and (sometimes) contradictory prescriptions for action can negatively influence safety-related thinking, decisions, and behaviours of organisational members (Chapter 4). These results demonstrate that seemingly irrational actions leading to safety incidents can have underlying rationales that make perfect sense at the given time and place to those involved. 
Third, safety communication is an important factor for improving occupational safety. The outcomes of the systematic literature review (Chapter 2) illustrated that the vast majority of research on the relationship between (safety) communication and occupational safety underlined the positive influence of (safety) communication on performance (28 out of 35 relationships), although the (more direct) relationship with negative safety outcomes was less clear ( 8 out of 16 relationships). Furthermore, safety communication was perceived as one of the most promising safety management instruments by supervisors (Chapter 3 ), further underlining its potential for improving occupational safety. Not only did the supervisors view safety communication as an important factor on its own, they also emphasised its importance for other safety management instruments such as inspections, accident registration and accident analysis (Chapter 3). Despite its importance, a clear and common agreed upon conceptualisation of how safety communication relates to other safety variables (e.g., safety climate, safety performance) is lacking. This is potentially problematic given the increasing importance of safety communication for occupational safety and organisations due to societal and organisational changes that are taking place (e.g., globalisation and flexibilisation of the labour market). In order to make maximum use of communication to improve occupational safety, current views on safety communication should be broadened. In addition to the (perceived) importance of communication for occupational safety (Chapter 2, Chapter 3 ), communication proved vital in uncovering the different, and sometimes conflicting, rationales for safety-related thinking, decisions, and behaviours (Chapter 4). Furthermore, the results from Chapter 6 illustrate that the focus on supervisor-subordinate communication in existing safety communication measurement scales are too narrow and that a more comprehensive view on safety communication could benefit the creation of safer workplaces.

\subsection{Practical implications}

The outcomes brought forward by the different studies in this dissertation have several implications for practice. In order to effectively address the complexity of occupational safety, which is illustrated on different levels, there is a need for an integrated approach. 
First, as occupational safety is illustrated to be subject to the dynamics of the organisation, practitioners should address workplace safety through a comprehensive approach that addresses both technical aspects and requirements, as well as cognitive, behavioural, social and organisational aspects (e.g., Daniellou et al., 2011; O’Dea \& Flin, 2001; Törner \& Pousette, 2009; Wachter \& Yorio, 2014). In addition to such a comprehensive approach, safety practitioners and managers should assure that the approach in their organisation is also coherent towards the safety objective. This implies that all organisational actors should be on the same page regarding the importance of occupational safety in relation to other organisational objectives and that organisational members should be provided with the means (e.g., time, money, authority) to maintain and improve occupational safety. Furthermore, top and higher management should strive for a high degree of compatibility between the prescriptions from the different institutional logics in order to minimise the adverse effects on safety of conflicting prescriptions for action. They can do so by assessing the different logics at play within their organisation, and subsequently clarify the position of safety in relation to these logics.

Second, to account for the influences from beyond the boundaries of the organisation itself, practitioners should attune the approaches within their organisation with other parties involved, including governmental agencies, clients and (sub)contractors. This way, the differences between organisational approaches to occupational safety can be minimised which is especially of importance considering the looser labour market and widespread use of freelancers and (sub)contractors. Additionally, adverse influences of the market (i.e. self-interested focus on increasing efficiency and profits) can be minimised. Governmental agencies should stimulate a comprehensive approach to occupational safety within organisations. They could do so by stimulating organisations to look beyond the technical requirements of the work environment, and underline the importance and the influence of cognitive, behavioural, social and organisational aspects of safety.

Third, in order to successfully improve occupational safety, practitioners are recommended to conduct a thorough analysis before implementing safety policies, interventions or safety management instruments. As the outcomes of the systematic literature review (Chapter 2) illustrate the lack of consensus regarding the effectiveness of a majority of factors in addressing safety outcomes and safety performance, practitioners should not trust blindly on 
the findings from a singular study. Such an approach could lead to unwanted or even problematic outcomes. Furthermore, even when the effectivity of a specific factor has been demonstrated in numerous scientific studies, practitioners are advised to take the perceptions and beliefs of employees into account (Chapter 3 ). When employees are unwilling to adopt and uphold safety policies, interventions or safety management instruments the chances of success are limited.

Lastly, the outcomes of the different studies presented here provide several directions for a more effective use of communication as a means to improve occupational safety. From the assessment of different levels of motivation and ability (Chapter 5) it follows that safety instructions should focus on a personal level of motivation and an external level of ability. Based on the outcomes of the safety communication measurement development, practitioners are recommended to pay attention to sender credibility in relation to safety motivation. To foster safety knowledge among their employees, practitioners are recommended to align their vertical communication, the tone used in their communications, and communication channels with the wants and needs of their employees, as well as assuring that safety communication reaches everyone within the organisation. Lastly, they should encourage open communication among co-workers in order to improve horizontal communication. Lastly, in order to come to an effective use of communication, practitioners should critically review current communication efforts within their organisation and compare them with the wants and needs of their target audiences. In doing so, they can identify (potential) barriers that prevent them from reaching employees.

\subsection{Limitations \& future research}

Aside from the limitations per study as mentioned and discussed in the various chapters, there are some overall limitations of the research reported in this dissertation.

First, while the general introduction (Chapter 1) and the systematic review of literature (Chapter 2) uncovered a wide variety of factors and approaches that can be used to address occupational safety, most of this dissertation deliberately focussed on a subset of these factors. The different studies in this 
dissertation focused on factors that organisational actors could influence. These factors were mainly those associated with management \& colleagues (see Table 2.5, p. 43). As a result, other factors were left out of consideration, despite their expected influence on occupational safety (Chapter 2). Future research should there for further study the influence and usability of the variables from the other clusters brought forward in the systematic literature review (Chapter 2). Potentially interesting areas include work characteristics (e.g., the importance of task clarity, finding the most optimal patterns for shift work, and the influence of different contract types), employee demographics (e.g., the influences of age, tenure / work experience, and work-life balance), and the influence of external factors (e.g., law \& legislation, and client involvement). In studying these areas and factors, researchers are encouraged to take the influences of the dynamics of the organisation into consideration.

Second, although most of the participants in the studies described in this dissertation were employed at internationally operating organisations, these organisations all originated from Western countries (Chapters 3, 4, 5, and 6). And although previous research on the influence of culture on occupational safety has not been conclusive (cf. Guldenmund, Cleal, \& Mearns, 2013; Lu, Lai, Venus Lun, \& Cheng, 2012; Mearns \& Yule, 2009; Starren, Hornikx, \& Luijters, 2013; Strauch, 2010) future research should validate the findings presented in this dissertation in different industries, organisational fields, and cultural contexts. In order to facilitate and encourage future researchers, the original (Dutch) items from the safety communication measurement scale (Chapter 6) have been translated to English using a forward-backward translation process. Both versions of the items are provided in the appendix (see Appendix B).

Third, the empirical studies described in this dissertation are all based on selfreports (Chapters 3, 4, 5 \& 6). Despite the wide-spread use of self-reported measures as an indication of actual attitudes and behaviours, self-reported measures (especially sensitive topics) can be susceptible for self-deception and impression management (Chan, 2010). Future research should therefore validate the findings from the studies presented here using alternative measurements such as observations and incident reports. Additionally, in order to assess the validity of previously conducted safety research based on self-reported data in general, researchers should assess the validity of self-reported measures of safety performance for actual safety performance. One way this could be done is through a longitudinal study where repeated 
measures of self-reported safety performance are coupled with observations or incidents reports. Such a study would both help future researchers as well as assess the quality of previous research findings.

In addition to these limitations and corresponding directions for future research, the findings from the studies presented in this dissertation provide several other directions for future research. First, future research should study occupational safety in relation to the dynamics of the organisation. The demonstrated influences on safety-related thinking, decisions and behaviours (Chapter 3, Chapter 4) illustrate the importance of a more comprehensive approach to occupational safety. By taking the various internal and external influences into account in studying occupational safety, current understandings of occupational safety could be further improved. Second, future research should further study the role and potential of communication in the improvement of occupational safety. While the outcomes of this dissertation already illustrated the (perceived) importance of communication for occupational safety and revealed the rather narrow view on safety communication in existing measurement scales, future research should focus on the development of 'best practices' concerning communication policies and interventions. Such policies should focus on the added value of motivational aspects (Chapter 5), and the use of communication for increasing safety knowledge (Chapter 6). Lastly, future research should explore if and how occupational safety could be improved by addressing associations and choice on subconscious levels, for example through the use of priming, framing or nudging. Priming is used to refer to a process in which sensory input (e.g., words or images) is used to activate specific mental constructs or schemas, which in turn affect thought processes, actions and behaviours (Hoekstra \& Wegman, 2011). Previous research on priming has demonstrated that it can be effectively used to affect risk preferences (Erb, Bioy, \& Hilton, 2002), and pro-environmental behaviours (Tate, Stewart, \& Daly, 2014). Framing refers to the process 'by which people develop a particular conceptualization of an issue or reorient their thinking about an issue' (Chong \& Druckman, 2007, p. 104). Previous research has demonstrated the impact of framing on safetyrelated rule violations (Von der Heyde, Brandhorst, \& Kluge, 2015), and on decision-making regarding health behaviours (Bartels, Kelly, \& Rothman, 2010). Lastly, nudging aims to influence people's choices and alter their behaviour by adapting aspects of the choice architecture, without forbidding or making it impossible to do otherwise (for more information see Thaler \& 
Sunstein, 2008). Nudges have been proposed already by others (e.g., Lindhout \& Reniers, 2017) to improve occupational safety and has been proved effective in increasing rule compliance among farmers (Peth, Mußhof, Funke, \& Hirschauer, 2018), and helping people to make securer decisions in connecting to wireless networks (Turland, Coventry, Jeske, Briggs, \& Van Moorsel, 2015).

\subsection{Conclusion}

Confronted with the enormous impact of incidents, accidents and injuries on employees, friends, family, colleagues, organisations and society, this dissertation aims to contribute to current scientific understanding of occupational safety to improve safety in the work context. An initial exploration of the occupational safety field led to the first research question in this dissertation:

\section{RQ1: What is the current status of occupational safety research with regard to determinants of safety outcomes and performance?}

The outcomes of the different studies in this dissertation illustrate that occupational safety is subject to the dynamics of the organisational environment, and that occupational safety covers a wide variety of different factors making it a complex issue that should be addressed through a comprehensive approach. Considering the importance of communication for the organisation and coordination of work, the central research question in this dissertation is:

\section{RQ2: What is the role of communication in occupational safety?}

The outcomes demonstrate that safety communication is one of the most promising management instruments for improving occupational safety within organisations. Not only did previous studies demonstrate its effectiveness (Chapter 2), managers also perceived communication to be of importance for other safety management instruments, including inspections and accident analysis (Chapter 3). Furthermore, communication proved vital for uncovering different and conflicting rationales for safety-related thinking, decisions, and 
behaviours (Chapter 4). Lastly, the results reveal that safety communication comprises more than supervisor-subordinate communication and is construed of a variety of aspects, which have strong relationships with safety climate, and -to a lesser extent- with safety motivation and safety knowledge (Chapter 6). 



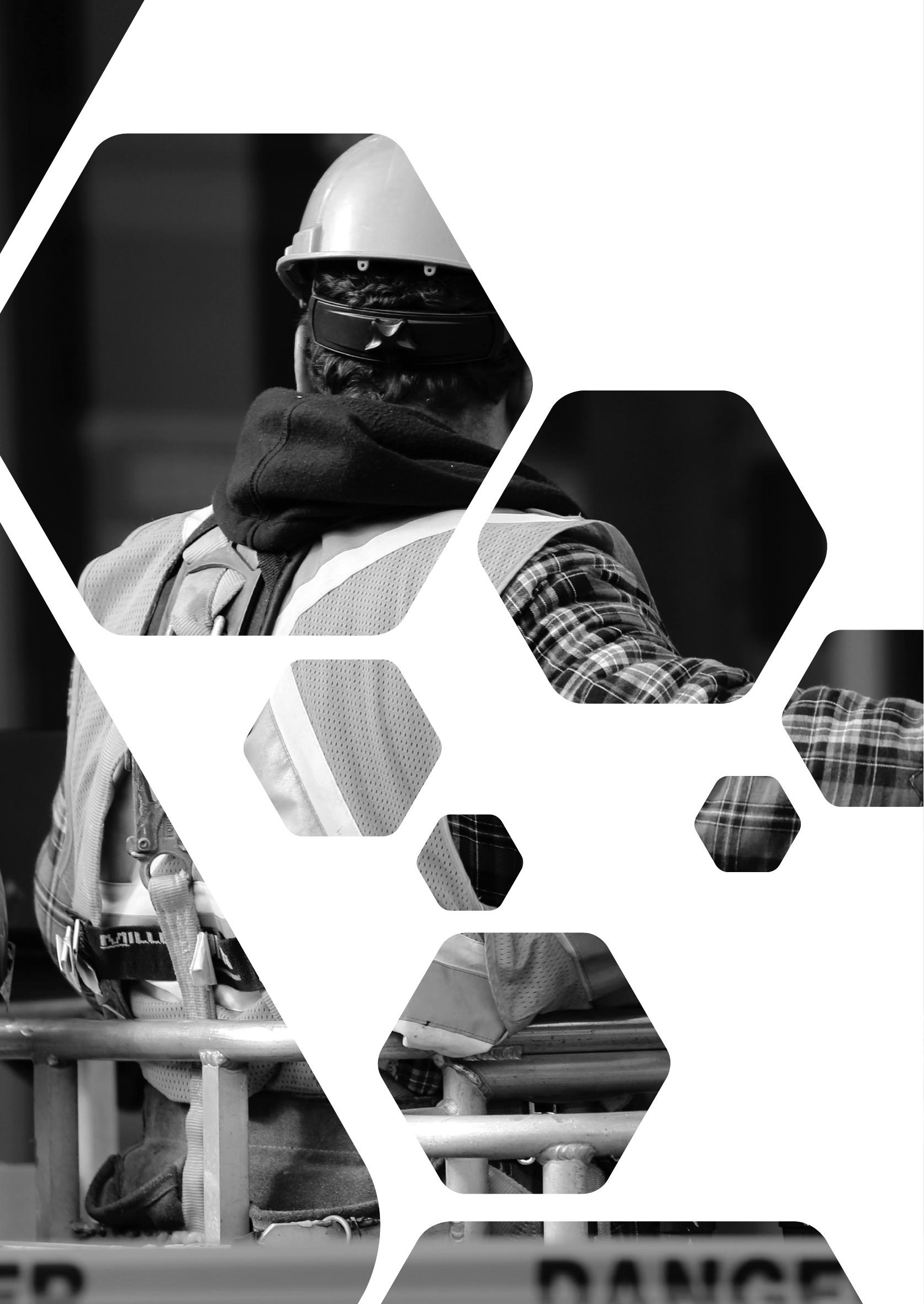




\section{References}




\section{References}

Abdul-Aziz, A.-R., \& Hussin, A.-A. (2003). Construction safety in Malaysia: A review of industry performance and outlook for the future. Journal of Construction Research, 4(2), 141-153. doi: 10.1142/S1609945103000364

Abudayyeh, O., Fredericks, T. K., Butt, S. E., \& Shaar, A. (2006). An investigation of management's commitment to construction safety. International Journal of Project Management, 24(2), 167-174. doi: 10.1016/j. ijproman.2005.07.005

Ahonen, E. Q., Benavides, F. G., \& Benach, J. (2007). Immigrant populations, work and health - A systematic literature review. Scandinavian Journal of Work, Environment \& Health, 33(2), 96-104.

Ajzen I. (1985) From intentions to actions: A theory of planned behavior. In: J. Kuhland \& J. Beckmann (Eds.), Action control: From cognitions to behavior (pp. 11-39). Heidelberg: Springer.

Ajzen, I. (1991). The theory of planned behavior. Organizational Behavior and Human Decision Processes, 50, 179-211.

Alarcón, L. F., Acuña, D., Diethelm, S., \& Pellicer, E. (2016). Strategies for improving safety performance in construction firms. Accident Analysis \& Prevention, 94, 107-118. doi: 10.1016/j.aap.2016.05.021

Alsamadani, R., Hallowell, M., \& Javernick-Will, A. M. (2013a). Measuring and modelling safety communication in small work crews in the US using social network analysis. Construction Management and Economics, 31(6), 568579. doi: 10.1080/01446193.2012.685486

Alsamadani, R., Hallowell, M. R., Javernick-Will, A., \& Cabello, J. (2013b). Relationships among language proficiency, communication patterns, and safety performance in small work crews in the United States. Journal of Construction Engineering and Management, 139(9), 1125-1134. doi: 10.1061/(ASCE)C0.1943-7862.0000724

Andriessen, J. H. T. H. (1978). Safe behaviour and safety motivation. Journal of Occupational Accidents, 1, 363-376.

Arbous, A. G., \& Kerrich, J. E. (1951). Accident statistics and the concept of accident-proneness. Biometrics, 7(4), 340-432. doi: 10.2307/3001656 
Axley, S. R. (1984). Managerial and organizational communication in terms of the conduit metaphor. The Academy of Management Review, 9(3), 428-437.

Bakker, A. B., Demerouti, E. (2007). The job demands-resources model: State of the art. Journal of Managerial Psychology, 22(3), 309-328. doi: $10.1108 / 02683940710733115$

Barling, J., Loughlin, C., \& Kelloway, E. K. (2002). Development and test of a model linking safety-specific transformational leadership and occupational safety. Journal of Applied Psychology, 87(3), 488-496. doi: 10.1037//00219010.87.3.488

Bartels, J., Peters, O., de Jong, M. D. T., Pruyn, A. T. H., \& van der Molen, M. (2010). Horizontal and vertical communication as determinants of professional and organisational identification. Personnel Review, 39(2), 210-226.

Bartels, R. D., Kelly, K. M., \& Rothman, A. J. (2010). Moving beyond the function of the health behaviour: The effect of message frame on behavioural decision-making. Psychology \& Health, 25(7), 821-838. doi: 10.1080/08870440902893708

Bartlett, M. S. (1954). A note on the multiplying factors for various $\chi$ 2 approximations. Journal of the Royal Statistical Society. Series B (Methodological), 296-298.

Basha, S. A., \& Maiti, J. (2013). Relationships of demographic factors, job risk perception and work injury in a steel plant in India. Safety Science, 51(1), 374-381. doi: 10.1016/j.ssci.2012.08.005

Behm, M., \& Schneller, A. (2013). Application of the Loughborough construction accident causation model: A framework for organizational learning. Construction Management and Economics, 31(6), 580-595. doi: 10.1080/01446193.2012.690884

Bentley, T. A., \& Haslam, R. A. (2001). Identification of risk factors and countermeasures for slip, trip and fall accidents during the delivery of mail. Applied Ergonomics, 32(2), 127-134.

Besharov, M. L., \& Smith, W. K. (2014). Multiple institutional logics in organizations: Explaining their varied nature and implications. Academy of Management Review, 39(3), 364-381. doi: 10.5465/amr.2011.0431 
Besnard, D., \& Hollnagel, E. (2014). I want to believe: Some myths about the management of industrial safety. Cognition, Technology \& Work, 16, 13-23. doi: 10.1007/s10111-012-0237-4

Bhappu, A. D. (2000). The Japanese family: An institutional logic for Japanese corporate networks and Japanese management. Academy of Management Review, 25(2), 409-415.

Bjerkan, A. M. (2010). Health, environment, safety culture and climate Analysing the relationships to occupational accidents. Journal of Risk Research, 13(4), 445-477. doi: 10.1080/13669870903346386

Boughaba, A., Hassane, C., \& Roukia, O. (2014). Safety culture assessment in petrochemical industry: A comparative study of two Algerian plants. Safety and Health at Work, 5(2), 60-65. doi: 10.1016/j.shaw.2014.03.005

Bray, S. R., Gyurcsik, N. C., Culos-Reed, S. N., Dawson, K. A., \& Martin, K. A. (2001). An exploratory investigation of the relationship between proxy efficacy, self-efficacy and exercise attendance. Journal of Health Psychology, 6(4), 425-434.

Brewer, N. T., Weinstein, N. D., Cuite, C. L., \& Herrington, Jr. J. E. (2004). Risk perceptions and their relation to risk behavior. Annals of Behavioral Medicine, 27(2), 125-130.

Burke, M. J., Sarpy, S. A., Tesluk, P. E., \& Smith-Crowe, K. (2002). General safety performance: A test of a grounded theoretical model. Personnel Psychology, 55, 429-457. doi: 10.1111/j.1744-6570.2002.tb00116.x

Cahill, L. B. (2010). Achieving environmental, health, and safety compliance in the United States: Hard and getting harder. Environmental Quality Management, 20(1), 1-7.

Catell, R.B. (1966). The scree test for number of factors. Multivariate Behavioral Research, 1(2), 245-276.

Cavazotte, F. S. C. N., Duarte, C. J. P., \& Gobbo, A. M. C. (2013). Authentic leader, safe work: The influence of leadership on safety performance. Brazilian Business Review, 10(2), 95-119.

Chan, D. (2010). So why ask me? Are self-report data really that bad? In C. E. Lance \& R. J. Vandenberg (Eds.), Statistiscal and Methodological Myths and Urban Legends (pp. 309-336). New York: Routledge. 
Cheng, C. W., Leu, S. S., Lin, C. C., \& Fan, C. (2010). Characteristic analysis of occupational accidents at small construction enterprises. Safety Science, 48, 698-707.

Cheyne, A., Cox, S., Oliver, A., \& Tomás, J. M. (1998). Modelling safety climate in the prediction of levels of safety activity. Work \& Stress, 12(3), 255-271. doi: $10.1080 / 02678379808256865$

Chinander, K.R., Kleindorfer, P. R., \& Kunreuther, H. C. (1998). Compliance strategies and regulatory effectiveness of performance-based regulation of chemical accident risks. Risk Analysis, 18(2), 135-143.

Chong, D., \& Druckman, J. N. (2007). Framing theory. Annual Review of Political Science 10, 103-126 doi: 10.1146/annurev.polisci.10.072805.103054

Choudhry, R. (2012). Implementation of BBS and the impact of site-level commitment. Journal of Professional Issues in Engineering, Education and Practice, 138(4), 296-304. doi: 10.1061/(ASCE)EI.1943-5541.0000111.

Choudhry, R. M., Fang, D., \& Mohamed, S. (2007). The nature of safety culture: A survey of the state-of-the-art. Safety Science, 45(10), 993-1012. doi: 10.1016/j.ssci.2006.09.003

Christian, M. S., Bradley, J. C., Wallace, J. C., \& Burke, M. J. (2009). Workplace safety: A meta-analysis of the roles of person and situation factors. Journal of Applied Psychology, 94(5), 1103-1127. doi: 10.1037/a0016172

Cigularov, K. P., Chen, P. Y., Rosecrance, J. (2010). The effects of error management climate and safety communication on safety: A multi-level study. Accident Analysis and Prevention, 42, 1498-1506. doi: 10.1016/j. aap.2010.01.003

Clarke, S. (1998). Organizational factors affecting the incident reporting of train drivers. Work \& Stress, 12(1), 6-16. doi: $10.1080 / 02678379808256845$

Clarke, S. (2006). The relationship between safety climate and safety performance: A meta-analytic review. Journal of Occupational Health Psychology, 11(4), 315-327. doi: 10.1037/1076-8998.11.4.315 
Clarke, S. (2013). Safety leadership: A meta-analytic review of transformational and transactional leadership styles as antecedents of safety behaviours. Journal of Occupational and Organizational Psychology, 86, 22-49. doi:10.1111/j.2044-8325.2012.02064.x

Clouder, L. (2009). 'Being responsible': Students' perspectives on trust, risk and work-based learning. Teaching in Higher Education, 14(3), 289-301. doi: $10.1080 / 13562510902898858$

Conchie, S. M., \& Burns, C. (2009). Improving occupational safety: Using a trusted information source to communicate about risk. Journal of Risk Research, 12(1), 13-25. doi: 10.1080/13669870802433749

Cooper, M. D., \& Philips, R. A. (2004). Exploratory analysis of the safety climate and safety behavior relationship. Journal of Safety Research, 35, 497-512.

Cooper, M. D., Philips, R. A., Sutherland, V. J., \& Makin, P. J. (1994). Reducing accidents using goal setting and feedback: A field study. Journal of Occupational and Organizational Psychology, 67, 219-240.

Cornelissen, P. A., Van Hoof, J. J., \& De Jong, M. D. T. (2017). Determinants of safety outcomes and performance: A systematic literature review of research in four high-risk industries. Journal of Safety Research, 62, 127141. doi: 10.1016/j.jsr.2017.06.009

Corvalan, C. F., Driscoll, T. R., \& Harrison, J. E. (1994). Role of migrant workers in work-related fatalities in Australia. Scandinavian Journal of Work, Environment \& Health, 20(5), 364-370. doi: 10.5271/sjweh.1385

Cox, S., \& Flin, R. (1998). Safety culture: Philosopher's stone or man of straw? Work \& Stress, 12(3), 189-201.

Coyle, I. R., Sleeman, S. D., \& Adams, N. (1995). Safety climate. Journal of Safety Research, 26(4), 247-254.

Daft, R.L., \& Lengel, R.H. (1986). Organizational information requirements, media richness and structural design. Management Science, 32(5), 554571. doi: $10.1287 /$ mnsc.32.5.554

Daniellou, F., Simard, M. \& Boissières, I. (2011). Human and organizational factors of safety: A state of the art. Number 2011-01 of the Cahiers de la Sécurité Industrielle, Foundation for an Industrial Safety Culture, Toulouse, France (ISSN 2100-3874). Available at http://www.FonCSI.org/en/ 
Daudigeos, T., Boutinot, A., \& Jaumier, S. (2013). Taking stock of institutional complexity: Anchoring a pool of institutional logics into the interinstitutional system with a descendent hierarchical analysis. In Institutional logics in action, part B (pp. 319-350). Emerald Group Publishing Limited.

De Cuyper, N., \& De Witte, H. (2007). Job insecurity in temporary versus permanent workers: Associations with attitudes, well-being, and behavior. Work \& Stress, 21(1), 65-84. doi: 10.1080/02678370701229050

Dedobbeleer, N., \& German, P. (1987). Safety practices in construction industry. Journal of Occupational Medicine, 29(11), 863-868.

DeHart-Davis, L., Chen, J., \& Little, T. D. (2013). Written versus unwritten rules: The role of rule formalization in green tape. International Public Management Journal, 16(3), 331-356. doi: 10.1080/10967494.2013.825193

DeJoy, D. M., Schaffer, B. S., Wilson, M. G., Vandenberg, R. J., \& Butts, M. M. (2004). Creating safer workplaces: Assessing the determinants and role of safety climate. Journal of Safety Research, 35, 81-90.

Dekker, S. W. A. (2003). Accidents are normal and human error does not exists: A new look at the creation of occupational safety. International Journal of Occupational Safety and Ergonomics, 9(2), 211-218. doi: 10.1080/10803548.2003.11076564

Díaz, R. I., \& Cabrera, D. D. (1997). Safety climate and attitude as evaluation measures of organizational safety. Accident Analysis and Prevention, 29(5), 643-650.

Döös, M., Backström, T., \& Sundström-Frisk, C. (2004). Human actions and errors in risk handling - An empirically grounded discussion of cognitive action-regulation levels. Safety Science, 42(3), 185-204. doi: 10.1016/ S0925-7535(03)00026-2

Dragga, S. (2011). Cooperation or compliance: Building dialogic codes of conduct. Technical Communication, 58(1), 4-18. 
Drupsteen, L., Groeneweg, J., \& Zwetsloot, G. I. J. M. (2013). Critical steps in learning from incidents: Using learning potential in the process from reporting an incident to accident prevention. International Journal of Occupational Safety and Ergonomics, 19(1), 63-77. doi: 10.1080/10803548.2013.11076966

Eakin, J. M. (1992). Leaving it up to the workers: Sociological perspectives on the management of health and safety in small workplaces. The International Journal of Health Services, 22(4), 689-704.

Elling, R. (1997). Revising safety instructions with focus groups. Journal of Business and Technical Communication, 11(4), 451-468.

Entman, R. M. (1993). Framing: Toward clarification of a fractured paradigm. Journal of Communication, 43(4), 51-58.

Erb, H-P., Bioy, A., \& Hilton, D. J. (2002). Choice preferences without inferences: Subconscious priming of risk attitudes. Journal of Behavioral Decision Making, 15, 251-262. doi: 10.1002/bdm.416

Eurostat. (2016). Retrieved December 20, 2016, from http://ec.europa.eu/ eurostat/statistics-explained/index.php/Accidents_at_work_statistics

Evans, S. M., Berry, J. G., Smith, B. J., Esterman, A., O’Shaughnessy, J., \& DeWit, M. (2006). Attitudes and barriers to incident reporting: A collaborative hospital study. Quality and Safety in Health Care, 15(1), 39-43. doi: 10.1136/qshc.2004.012559

Fairclough, S., \& Micelotta, E. R. (2013). Beyond the family firm: Reasserting the influence of the family institutional logic across organizations. In Institutional logics in Action, Part B (pp. 63-89). Emerald Group Publishing Limited.

Farr, J. (2004). Social capital: A conceptual history. Political Theory, 32(1), 6-33.

Fellner, D. J., \& Sulzer-Azaroff, B. (1984). Increasing industrial safety practices and conditions through posted feedback. Journal of Safety Research, 15(1), 7-21. doi: 10.1016/0022-4375(84)90026-4 
Fernández-Muñiz, B., Montes-Peón, J. M., \& Vázquez-Ordás, C. J. (2012).

Safety climate in OHSAS 18001-certified organisations: Antecedents and consequences of safety behaviour. Accident Analysis and Prevention, 45, 745-758. doi: 10.1016/j.aap.2011.10.002

Field, A. (2009). Discovering statistics using SPSS ( $3^{\text {rd }}$ Edition). London, United Kingdom: SAGE Publications Ltd.

Fischer, P., Krueger, J. I., Greitemeyer, T., Vogrincic, C., Kastenmüller, A., Frey, D., Heene, M., Wicher, M., \& Kainbacher, M. (2011). The bystander-effect: A meta-analytic review on bystander intervention in dangerous and non-dangerous emergencies. Psychological Bulletin, 137(4), 517-537. doi: $10.1037 / \mathrm{a} 0023304$

Fishbein, M., \& Ajzen, I. (1975). Belief, attitude, intention, and behavior: An introduction to theory and research. Reading, MA: Addison-Wesley.

Frewer, L. J., \& Miles, S. (2003). Temporal stability of the psychological determinants of trust: Implications for communication about food risks. Health, Risk \& Society, 5(3), 259-271. doi: $10.1080 / 13698570310001606969$

Friedland, R. (2001). Religious nationalism and the problem of collective representation. Annual Review of Sociology, 27, 125-152.

Friedland, R. (2002). Money, sex, and god: The erotic logic of religious nationalism. Sociological theory, 20(3), 381-425.

Friedland, R., \& Alford, R. R. (1991). Bringing society back in: Symbols, practices and institutional contradictions. In W. W. Powell \& P. J. DiMaggio (Eds.), The new institutionalism in organizational analysis (232-267). Chicago, IL: University of Chicago Press.

Geller, S. E. (2005). Behavior-based safety and occupational risk management. Behavior Modification, 29(3), 539-561. doi: 10.1177/0145445504273287

Ghosh, S. (2014). Does formal daily huddle meetings improve safety awareness? International Journal of Construction Education and Research, 10(4), 285-299. doi: 10.1080/15578771.2014.886642 
Glynn, M. A., \& Lounsbury, M. (2005). From the critics' corner: Logic blending, discursive change and authenticity in a cultural production system. Journal of Management Studies, 42(5), 1031-1055. doi: 10.1111/j.14676486.2005.00531.x

Goodrick, E., \& Reay, T. (2011). Constellations of institutional logics: Changes in the professional work of pharmacists. Work and Occupations, 38(3), 372416. doi: $10.1177 / 0730888411406824$

Grabowski, M., Ayyalasomayajula, P., Merrick, J., \& Mccafferty, D. (2007). Accident precursors and safety nets: Leading indicators of tanker operations safety. Maritime Policy \& Management, 34(5), 405-425. doi: 10.1080/03088830701585084

Gray, G. C. (2006). The regulation of corporate violations: Punishment, compliance, and the blurring of responsibility. The British Journal of Criminology, 46(5), 875-892. doi: 10.1093/bjc/azl005

Gray, G. C. (2009). The responsibilization strategy of health and safety: Neoliberalism and the reconfiguration of individual responsibility for risk. The British Journal of Criminology, 39(3), 326-342. doi: 10.1093/bjc/azp004

Greenwood, R., Díaz, A. M., Li, S. X., \& Lorente, J. C. (2010). The multiplicity of institutional logics and the heterogeneity of organizational responses. Organization Science, 21(2), 521-539. doi: 10.1287/orsc.1090.0453

Greenwood, R., Raynard, M., Kodeih, F., Micelotta, E. R., \& Lounsbury, M. (2011). Institutional complexity and organizational responses. The Academy of Management Annals, 5(1), 317-371. doi: 10.1080/19416520.2011.590299

Guldenmund, F. W. (2000). The nature of safety culture: A review of theory and research. Safety Science, 34, 215-257. doi: 10.1016/S09257535(00)00014-X

Guldenmund, F. W. (2007). The use of questionnaires in safety culture research - An evaluation. Safety Science, 45, 723-743. doi: 10.1016/j. ssci.2007.04.006

Guldenmund, F., Cleal, B., \& Mearns, K. (2013). An exploratory study of migrant workers and safety in three European countries. Safety Science, 52, 92-99. doi: 10.1016/j.ssci.2012.05.004 
Gyekye, S. A. (2005). Workers' perceptions of workplace safety and job satisfaction. International Journal of Occupational Safety and Ergonomics, 11(3), 291-302.

Gyekye, S. A., \& Salminen, S. (2009). Educational status and organizational safety climate: Does educational attainment influence workers' perceptions of workplace safety? Safety Science, 47, 20-28.

Hahn, S. E., \& Murphy, L. R. (2008). A short scale for measuring safety climate. Safety Science, 46(7), 1047-1066. doi: 10.1016/j.ssci.2007.06.002

Hale, A. R. (1990). Safety rules O.K.? Possibilities and limitations in behavioural safety strategies. Journal of Occupational Accidents, 12, 3-20.

Hale, A., \& Borys, D. (2013). Working to rule, or working safely? Part 1: A state of the art review. Safety Science, 55, 207-221. doi: 10.1016/j. ssci.2012.05.011

Hale, A., \& Borys, D. (2013). Working to rule or working safely? Part 2: The management of safety rules and procedures. Safety Science, 55, 222-231.

Harvey, J., Bolam, H., Gregory, D., \& Erdos, G. (2001). The effectiveness of training to change safety culture and attitudes within a highly regulated environment. Personnel Review, 30(6), 615-636.

Hasan, A., \& Jha, K. N. (2013). Safety incentive and penalty provisions in Indian construction projects and their impact on safety performance. International Journal of Injury Control and Safety promotion, 20(1), 3-12. doi: 10.1080/17457300.2011.648676

Håvold, J. I., \& Nesset (2009). From safety culture to safety orientation: Validation and simplification of a safety orientation scale using a sample of seafarers working for Norwegian ship owners. Safety Science, 47, 305-326. doi: 10.1016/j.ssci.2008.05.002

Hayes, B. E., Perander, J., Smecko, T., \& Trask, J. (1998). Measuring perceptions of workplace safety: Development and validation of the work safety scale. Journal of Safety Research, 29(3), 145-161.

Heinrich, H.W. (1931). Industrial accident prevention: A scientific approach. New York, NY: McGraw-Hill. 
Herrerro, S. G., Saldaña, M. A. M., Del Campo, M. A. M., \& Ritzel, D. O. (2002). From the traditional concept of safety management to safety integrated with quality. Journal of Safety Research, 33, 1-20.

Heslin, P. A. (2009). Better than brainstorming? Potential contextual boundary conditions to brainwriting for idea generation in organizations. Journal of Occupational and Organizational Psychology, 82, 129-145. doi: 10.1348/096317908X285642

Hinze, J., Thurman, S., \& Wehle, A. (2013). Leading indicators of construction safety performance. Safety Science, 51(1), 23-28. doi: 10.1016/j. ssci.2012.05.016

Hoekstra, T., \& Wegman, F. (2011). Improving the effectiveness of road safety campaigns: Current and new practices. International Association of Traffic and Safety Sciences, 34, 80-86. doi: 10.1016/j.iatssr.2011.01.003

Hofmann, D. A., \& Morgeson, F. P. (1999). Safety-related behavior as a social exchange: The role of perceived organizational support and leadermember exchange. Journal of Applied Psychology, 84(2), 286-296.

Hofmann, D. A., \& Stetzer, A. (1998). The role of safety climate and communication in accident interpretation: Implications for learning from negative events. Academy of Management Journal, 41(6), 644-657.

Hofmann, D. A., Jacobs, R., \& Landy, F. (1995). High reliability process industries: Individual, micro, and macro organizational influences on safety performance. Journal of Safety Research, 26(3), 131-149.

Hofmann, D. A., Morgeson, F. P., \& Gerras, S. J. (2003). Climate as a moderator of the relationship between leader-member exchange and content specific citizenship: Safety climate as an exemplar. Journal of Applied Psychology, 88(1), 170-178. doi: 10.1037/0021-9010.88.1.170

Hopkins, A. (1999). For whom does safety pay? The case of major accidents. Safety Science, 32(2-3), 143-153. doi: 10.1016/S0925-7535(99)00017-X

Huang, H. J. (1999). Job rotation from the employees' point of view. Research and Practice in Human Resource Management, 7(1), 75-85.

Incident [Def. 1]. In Oxford Advanced Learner's Dictionary $7^{\text {th }}$ edition, Retrieved April 16 ${ }^{\text {th }}, 2018$. 
Ingram, P., \& Rao, H. (2004). Store wars: The enactment and repeal of antichain-store legislation in America. American Journal of Sociology, 110(2), 446-487. doi: 10.1086/422928

Ingram, P., Yue, L., Q., \& Rao, H. (2010). Trouble in store: Probes, protests, and store openings by Wal-Mart, 1998-2007. American Journal of Sociology, 116(1), 53-92. doi: 10.1086/653596

Inspectorate SZW. (2016). Retrieved December 20, 2016, from https://www. inspectieszw.nl/publicaties/sectoraanpak/

Institute of Medicine (US) Committee to assess training needs for occupational safety and health personnel in the United States (2000). Safe Work in the $21^{\text {st }}$ Century: Education and Training Needs for the Next Decade's Occupational Safety and Health Personnel. Washington (DC): National Academies Press (US).

International Labour Organization. (1998). Resolution concerning statistics of occupational injuries (resulting from occupational accidents), adopted by the Sixteenth International Conference of Labour Statisticians (October 1998) [PDF file]. Retrieved from http://ilo.org/wcmsp5/groups/public/--dgreports/---stat/documents/normativeinstrument/wcms_087528.pdf

International Labour Organization. (1998). Technical and ethical guidelines for workers' health surveillance. Retrieved from http://www.ilo.org/ wcmsp5/groups/public/---ed_protect/---protrav/---safework/documents/ normativeinstrument/wcms_177384.pdf

International Labour Organization. (2010). Improving safety and health at work. Retrieved from http://www.ilo.org/safework/projects/ WCMS_149466/lang--en/index.htm

International Labour Organization. (2013). Safety and health at work. Retrieved from http://www.ilo.org/global/topics/safety-and-health-atwork/lang--en/index.htm

International Labour Organization. (2014). Occupational injuries. Retrieved from http://www.ilo.org/ilostat/faces/home/statisticaldata/data_by_ subject/subject-details?subject=INJ\&_afrLoop=760191095069219\&_adf. ctrl-state=t3qhz9mko_4 
International Labour Organization. (2015). Retrieved April $8^{\text {th }}$, 2015, from http://ilo.org/global/topics/safety-and-health-at-work/lang--en/index. htm

International Labour Organization. (2018). Retrieved May $4^{\text {th }}, 2018$, from http://www.ilo.org/global/topics/safety-and-health-at-work/lang--en/ index.htm

Jiang, L., Yu, G., Li, Y., \& Li, F. (2010). Perceived colleagues' safety knowledge/ behavior and safety performance: Safety climate as a moderator in a multilevel study. Accident Analysis and Prevention, 42, 1468-1476.

Johansen, C. B., \& Waldorff, S. B. (2015). What are institutional logics - And where is the perspective taking us?, Paper for the Academy of Management Annual Meeting, Vancouver, 2015.

Jones, C., Livne-Tarandach, R., \& Balachandra, L. (2010). Rhetoric that wins clients: Entrepreneurial firms use of institutional logics when competing for resources. Institutions and Entrepeneurship, 21, 183-218. doi: 10.1108/ S0277-2833(2010)0000021011

Kaiser, F. G., Ranney, M., Hartig, T., \& Bowler, P. A. (1999). Ecological behavior, environmental attitude, and feelings of responsibility for the environment. European Psychologist, 4(2), 59-74.

Kaiser, H. F. (1970). A second generation Little Jiffy. Psychometrika, 35(4), 401415.

Katz, E. (1957). The two-step flow of communication: An up-to-date report on a hypothesis. Public Opinion Quarterly, 21(1), 61-78.

Kelloway, E. K., Mullen, J., \& Francis, L. (2006). Divergent effects of transformational and passive leadership on employee safety. Journal of Occupational Health Psychology, 11(1), 76-86. doi: 10.1037/10768998.11.1.76

Khanzode, V. V., Maitei, J., \& Ray, P. K. (2012). Occupational injury and accident research: A comprehensive review. Safety Science, 50, 1355-1367.

Ko, K., Mendeloff, J., \& Gray, W. (2010). The role of inspection sequence in compliance with the US occupational safety and health administration's (OSHA) standards: Interpretations and implications. Regulation \& Governance, 4, 48-70. doi: 10.1111/j.1748-5991.2010.01070.x 
Kringos, D. S., Boerma, W. G. W., Hutchinson, A., Van der Zee, J., \& Groenewegen, P. P. (2010). The breadth of primary care: A systematic literature review of its core dimensions. BMC Health Services Research, 10(65), 1-13. doi: 10.1186/1472-6963-10-65

LaCroix, D. V., \& DeJoy, D. M. (1989). Causal attributions to effort and supervisory response to workplace accidents. Journal of Occupational Accidents, 11, 97-109.

Laitinen, H., \& Ruohomäki, I. (1996). The effects of feedback and goal setting on safety performance at two construction sites. Safety Science, 24(1), 6173.

Ledeneva, A. (2001). Unwritten Rules: How Russia Really Works. London: Center for European Reform.

Lee, K., Shon, D., \& Oah, S. (2014). The relative effects of global and specific feedback on safety behaviors. Journal of Organizational Behavior Management, 34(1), 16-28. doi: 10.1080/01608061.2013.878264

Lindhout, P., \& Reniers, G. (2017). What about nudges in the process industry? Exploring a new safety management tool. Journal of Loss Prevention in the Process Industries, 50 (Part A), 243-256. doi: 10.1016/j.jlp.2017.10.006

Lu, C-S., Lai, K-H, Venus Lun, Y. H., \& Cheng, T. C. E. (2012). Effects of national culture on human failures in container shipping: The moderating role of Confucian dynamism. Accident Analysis \& Prevention, 49, 457-469. doi: 10.1016/j.aap.2012.03.018

Luria, G. (2010). The social aspect of safety management: Trust and safety climate. Accident Analysis and Prevention, 42, 1288-1295. doi: 10.1016/j. aap.2010.02.006

MacCallum, R. C., Widaman, K. F., Zhang, S., \& Hong, S. (1999). Sample size in factor analysis. Psychological Methods, 4(1), 84-99.

Madden, T. J., Ellen, P. S., \& Ajzen, I. (1992). A comparison of the theory of planned behavior and the theory of reasoned action. Personality and Social Psychology Bulletin, 18(1), 3-9.

Madsen, C. U., \& Hasle, P. (2017). Commitment or compliance? Institutional logics of work environment management. Nordic Journal of Working Life Studies, 7, 17-38. 
Manuele, F. A. (2011). Reviewing Heinrich: Dislodging two myths from the practice of safety. Professional Safety, 56(10), 52-61.

Marshall, P., Hirmas, A., \& Singer, M. (2018). Heinrich's pyramid and occupational safety: A statistical validation methodology. Safety Science, 101, 180-189. doi: 10.1016/j.ssci.2017.09.005

Mayhew, C., Quintan, M., \& Ferris, R. (1997). The effects of subcontracting / outsourcing on occupational health and safety: Survey evidence from four Australian industries. Safety Science, 25(1-3), 163-178. doi: 10.1016/ S0925-7535(97)00014-3

Mearns, K., \& Yule, S. (2009). The role of national culture in determining safety performance: Challenges for the global oil and gas industry. Safety Science, 47, 777-785. doi: 10.1016/j.ssci.2008.01.009

Mearns, K., Whitaker, S. M., \& Flin, R. (2003). Safety climate, safety management practices and safety performance in offshore environments. Safety Science, 41, 641-680.

Melamed, S., \& Oksenberg, A. (2002). Excessive daytime sleepiness and risk of occupational injuries in non-shift daytime workers. Sleep, 25(3), 315-322.

Mengolini, A., \& Debarberis, L. (2008). Effectiveness evaluation methodology for safety processes to enhance organizational culture in hazardous installations. Journal of Hazardous Materials, 155(1-2), 243-252. doi: 10.1016/j.jhazmat.2007.11.078

Meyer, J. W., \& Rowan, B. (1977). Institutionalised organizations: Formal structure as myth and ceremony. American Journal of Sociology, 83(2), 340363.

Michael, J. H., Guo, Z. G., Wiedenbeck, J. K., \& Ray, C. D. (2006). Production supervisor impacts on subordinates' safety outcomes: An investigation of leader-member exchange and safety communication. Journal of Safety Research, 37(5), 469-477. doi: 10.1016/j.jsr.2006.06.004

Milgram, S. (1963). Behavioral study of obedience. Journal of Abnormal and Social Psychology, 67(4), 371-378. 
Mohamed, S. (2003). Scorecard approach to benchmarking organizational safety culture in construction. Journal of construction engineering and management, 129(1), 80-88. doi: 10.1061/(ASCE)07339364(2003)129:1(80)

Muecke, S. (2005). Effects of rotating night shifts: Literature review. Journal of Advanced Nursing, 50(4), 433-439. doi: 10.1111/j.13652648.2005.03409.x

Mullen, J. (2004). Investigating factors that influence individual safety behavior at work. Journal of Safety Research, 35(3), 275-285. doi: 10.1016/j.jsr.2004.03.011

Neal, A., \& Griffin, M. A. (2002). Safety climate and safety behaviour. Australian Journal of Management, 27, 67-76. doi: 10.1177/031289620202701S08

Neal, A., \& Griffin, M. A. (2006). A study of the lagged relationships among safety climate, safety motivation, safety behavior, and accidents at the individual and group levels. Journal of Applied Psychology, 91(4), 946-953. doi: 10.1037/0021-9010.91.4.946

Neal, A., Griffin, M. A., \& Hart, P. M. (2000). The impact of organizational climate on safety climate and individual behavior. Safety Science, 34(1-3), 99-109. doi: 10.1016/S0925-7535(00)00008-4

Nicolini, D., Delmestri, G., Goodrick, E., Reay, T., Lindberg, K., \& Adolfsson, P. (2016). Look what's back! Institutional complexity, reversibility and the knotting of logics. British Journal of Management, 27, 228-248. doi: 10.1111/1467-8551.12156

Nilsen, T. R. (1957). On defining communication. The Speech Teacher, 6(1), 1017. doi: $10.1080 / 03634525709376841$

Nöhammer, E., Schusterschitz, C., \& Stummer, H. (2010). Determinants of employee participation in workplace health promotion. International Journal of Workplace Health Management, 3(2), 97-110. doi: 10.1108/17538351011055005

O’Neill, S., Flanagan, J., \& Clarke, K. (2016). Safewash! Risk attenuation and the (mis) reporting of corporate safety performance to investors. Safety Science, 83, 114-130. doi: 10.1016/j.ssci.2015.11.007 
O'Dea, A., \& Flin, R. (2001). Site managers and safety leadership in the offshore oil and gas industry. Safety Science, 37(1), 39-57. doi: 10.1016/S09257535(00)00049-7

OSHA. (n.d.). Class 3 - Risk assessment and accident investigation [PowerPoint slides]. Retrieved from https://www.osha.gov/dte/grant_materials/fy11/ sh-22224-11/3_Risk_Management.pptx

Pahnke, E. C., Katila, R., \& Eisenhardt, K. M. (2015). Who takes you to the dance? How partners' institutional logics influence innovation in young firms. Administrative Science Quarterly, 60(4), 596-633. doi: $10.1177 / 0001839215592913$

Pallant, J. (2007). SPSS survival manual - A step by step guide to data analysis using SPSS for Windows ( $3^{\text {rd }}$ ed.). Maidenhead: Open University Press.

Pallant, J. (2011). SPSS survival manual: A step by step guide to data analysis using SPSS. Crows Nest, IN: Allen \& Unwin.

Paté-Cornell, M. E. (1990). Organizational aspects of engineering system safety: The case of offshore platforms. Science, 250(4985), 1210-1217. doi: 10.1126/science.250.4985.1210

Pawłowska, Z. (2015). Using lagging and leading indicators for the evaluation of occupational safety and health performance in industry. International Journal of Occupational Safety and Ergonomics, 21(3), 284-290. doi: 10.1080/10803548.2015.1081769

Peth, D., Mußhof, O., Funke, K., \& Hirschauer, N. (2018). Nudging farmers to comply with water protection rules - Experimental evidence from Germany. Ecological Economics, 152, 310-321. doi: 10.1016/j. ecolecon.2018.06.007

Pettersson-Strömbäck, A., Liljelind, I., Neely, G., \& Järvholm, B. (2008). Workers' interpretation of self-assessment of exposure. Annals of Occupational Hygiene, 52(7), 663-671. doi: 10.1093/annhyg/men042

Podgórski, D. (2000). Occupational health and safety management in Polish enterprises implementing total quality management systems. International Journal of Occupational Safety and Ergonomics, 6(sup1), 85-101. doi: $10.1080 / 10803548.2000 .11105110$ 
Ponnet, K., Reniers, G., \& Kempeneers, A. (2015). The association between students' characteristics and their reading and following safety instructions. Safety Science, 71, 56-60. doi: 10.1016/j.ssci.2014.07.001

Pousette, A., Larsson, S., \& Törner, M. (2008). Safety climate cross-validation, strength and prediction of safety behavior. Safety Science, 46, 398-404.

Price, M., Handley, K., Millar, J., \& O’Donovan, B. (2010). Feedback: All that effort, what is the effect? Assessment \& Evaluation in Higher Education, 35(3), 277-289. doi: 10.1080/02602930903541007

Probst, T. M., \& Estrada, A. X. (2010). Accident under-reporting among employees: Testing the moderating influence of psychological safety climate and supervisor enforcement of safety practices. Accident Analysis \& Prevention, 42(5), 1438-1444. doi: 10.1016/j.aap.2009.06.027

Quinlan, M. (1999). The implications of labour market restructuring in industrialized societies for occupational health and safety. Economic and Industrial Democracy, 20, 427-460.

Quinn, R. W., \& Dutton, J. E., (2005). Coordination as energy-in-conversation. Academy of Management Review, 30(1), 36-57. doi: 10.5465/

AMR.2005.15281422

Racicot, B. M., \& Wogalter, M. S. (1995). Effects of a video warning sign and social modelling on behavioral compliance. Accident Analysis and Prevention, 27(1), 57-64.

Reader, T. W., \& O'Connor, P. (2013). The Deepwater Horizon explosion: Nontechnical skills, safety culture, and system complexity. Journal of Risk Research, 17(3), 405-424. doi: 10.1080/13669877.2013.815652

Reay, T., \& Jones, C. (2016). Qualitatively capturing institutional logics. Strategic Organization, 14(4), 1-14. doi: 10.1177/1476127015589981

Reay, T., Jaskiewicz, P., \& Hinings, C. R. (2015). How family, business, and community logics shape family firm behavior and "rules of the game" in an organizational field. Family Business Review, 28(4), 292-311. doi: $10.1177 / 0894486515577513$

Reynaud, B. (2005). The void at the heart of rules: routines in the context of rule-following. The case of the Paris Metro Workshop. Industrial and Corporate Change, 14(5), 847-871. doi: 10.1093/icc/dth073 
Rousseau, D. M., \& Libuser, C. (1997). Contingent workers in high risk environments. California Management Review, 39(2), 103-123.

Rundmo, T., \& Hale, A. R. (2003). Managers' attitudes towards safety and accident prevention. Safety Science, 41(7), 557-574. doi: 10.1016/S09257535(01)00091-1

Saari, J. (1982). Long-term development of occupational accidents in Finland. Scandinavian Journal of Work, Environment \& Health, 8(2), 85-93. doi: 10.5271/sjweh.2484

Safety and health at work. (n.d.) Retrieved from: http://www.ilo.org/global/ topics/safety-and-health-at-work/lang--en/index.htm

Saleh, T. A. (2011). Testing the effectiveness of visual aids in chemical safety training. Journal of Chemical Health and Safety, 18(2), 3-8.

Sargeant, M., \& Tucker, E. (2009). Layers of vulnerability in occupational health and safety for migrant workers: Case studies from Canada and the United Kingdom. Comparative Research in Law \& Political Economy. Research Paper No. 8. Retrieved from http://digitalcommons.osgoode.yorku.ca/ clpe/123

Schwartz, B., \& Sharpe, K. (2010). Practical wisdom: The right way to do the right thing. London, UK: Penguin.

Shannon, C.E., \& Weaver, W. (1949). The mathematical theory of communication. Urbana: University of Illinois Press.

Sheeran, P. (2002). Intention-behavior relations: A conceptual and empirical review. European Review of Social Psychology, 12(1), 1-36.

Silva, S., Lima, M. L., \& Baptista, C. (2004). OSCI: An organizational and safety climate inventory. Safety Science, 42, 205-220.

Siu, O. L., Phillips, D. R., \& Leung, T. W. (2003). Age differences in safety attitudes and safety performance in Hong Kong construction workers. Journal of Safety Research, 34(2), 199-205. doi: 10.1016/S00224375(02)00072-5

Siu, O-L., Philips, D. R., Leung, T-W. (2004). Safety climate and safety performance among construction workers in Hong Kong: The role of psychological strains as mediators. Accident Analysis \& Prevention, 36(4), 359-366. doi: 10.1016/S0001-4575(03)00016-2 
Sneddon, A., Mearns, K., \& Flin, R. (2013). Stress, fatigue, situation awareness and safety in offshore drilling crews. Safety Science, 56, 80-88. doi: 10.1016/j.ssci.2012.05.027

Sonderegger, A., \& Sauer, J. (2010). The influence of design aesthetics in usability testing: Effects on user performance and perceived usability. Applied Ergonomics, 41, 403-410.

Song, X. M., Xie, J., \& Dyer, B. (2000). Antencedents and consequences of marketing managers' conflict-handling behaviors. Journal of Marketing, 64(1), 50-66. doi: 10.1509/jmkg.64.1.50.17989

Starren, A., Hornikx, J., \& Luijters, K. (2013). Occupational safety in multicultural teams and organizations: A research agenda. Safety Science, 52, 43-49. doi: 10.1016/j.ssci.2012.03.013

Statistics Netherlands. (2014). Arbeidsomstandigheden werknemers; geslacht en leeftijd. Retrieved from http://statline.cbs.nl/StatWeb/publication/ default.aspx?DM=SLNL\&PA=71204NED\&D1=39-45\&D2=0\&D3=a\&D4=a\& $\mathrm{HDR}=\mathrm{G} 3 \& \mathrm{STB}=\mathrm{G} 1 \% 2 \mathrm{cG} 2 \% 2 \mathrm{cT} \& \mathrm{VW}=\mathrm{T}$

Strauch, B. (2010). Can cultural differences lead to accidents? Team cultural differences and sociotechnical system operations. Human Factors, 52(2), 246-263. doi: 10.1177/0018720810362238

Swuste, P., van Gulijk, C., \& Zwaard, W. (2010). Safety metaphors and theories, a review of the occupational safety literature of the US, UK and The Netherlands, till the first part of the $20^{\text {th }}$ century. Safety Science, $48(8)$, 1000-1018. doi: 10.1016/j.ssci.2010.01.020

Swuste, P., Van Gulijk, C., Zwaard, W., \& Oostendorp, Y. (2014). Occupational safety theories, models and metaphors in the three decades since World War II, in the United States, Britain and the Netherlands: A literature review. Safety Science, 62, 16-27. doi: 10.1016/j.ssci.2013.07.015

Swuste, P., Van Gulijk, C., Zwaard, W., Lemkowitz, S., Oostendorp, Y., \& Groeneweg, J. (2016). Developments in the safety science domain, in the fields of general and safety management between 1970 and 1979, the year of the near disaster on Three Mile Island, a literature review. Safety Science, 86, 10-26. doi: 10.1016/j.ssci.2016.01.022 
Tate, K., Stewart, A. J., \& Daly, M. (2014). Influencing green behaviour through environmental goal priming: The mediating role of automatic evaluation. Journal of Environmental Psychology, 38, 225-232. doi: 10.1016/j. jenvp.2014.02.004

Thaler, R. H., \& Sunstein, C. R. (2008). Nudge: Improving decisions about health, wealth and happiness. New Haven, CT: Yale University Press.

Thompson, R. C., Hilton, T. F., \& Witt, L. A. (1998). Where the safety rubber meets the shop floor: A confirmatory model of management influence on workplace safety. Journal of Safety Research, 29(1), 15-24. doi: 10.1016/ S0022-4375(97)00025-X

Thornton, P. H. (2002). The rise of the corporation in a craft industry: Conflict and conformity in institutional logics. The Academy of Management Journal, 45(1), 81-101.

Thornton, P. H. (2004). Markets from culture: Institutional logics and organizational decisions in higher education publishing. Redwood City, CA: Stanford University Press.

Thornton, P. H., \& Ocasio, W. (1999). Institutional logics and the historical contingency of power in organizations: Executive succession in the higher education publishing industry, 1958-1990. American Journal of Sociology, 15(3), 801-843.

Thornton, P. H., \& Ocasio, W. (2008). Institutional logics. In R. Greenwood, C. Oliver, R. Suddaby, \& K. Sahlin (Eds.), The SAGE Handbook of Organizational Institutionalism. Retrieved from: https://pdfs.semanticscholar.org/ a1d0/6e0d361d133bc4b53972e83822a05879cf79.pdf

Thornton, P. H., Ocasio, W., \& Lounsbury, M. (2013). The institutional logics perspective: A new approach to culture, structure, and process. Oxford University Press on Demand.

Törner, M., \& Pousette, A. (2009). Safety in construction - A comprehensive description of the characteristics of high safety standards in construction work, from the combined perspective of supervisors and experienced workers. Journal of Safety Research, 40(6), 399-409. doi: 10.1016/j. jsr.2009.09.005 
Tracey, P. (2012). Religion and organization: A critical review of current trends and future directions. The Academy of Management Annals, 6(1), 87-134. doi: 10.1080/19416520.2012.660761

Tucker, S., Chmiel, N., Turner, N., Hershcovis, M. S., \& Stride, C. B. (2008). Perceived organizational support for safety and employee safety voice: The mediating role of co-worker support for safety. Journal of Occupational Health Psychology, 13(4), 319-330. doi: 10.1037/1076-8998.13.4.319

Turland, J., Coventry, L., Jeske, D., Briggs, P., \& van Moorsel, A. (2015, July). Nudging towards security: Developing an application for wireless network selection for android phones. In Proceedings of the 2015 British HCI conference (pp. 193-201). ACM.

Turner, N., Chmiel, N., \& Walls, M. (2005). Railing for safety: Job demands, job control, and safety citizenship role definition. Journal of Occupational Health Psychology, 10(4), 504-512. doi: 10.1037/1076-8998.10.4.504

Turner, N., Stride, C. B., Carter, A. J., McCaughey, D., \& Carroll, A. E. (2012). Job demands-control-support model and employee safety performance. Accident Analysis and Prevention, 45, 811-817. doi: 10.1016/j. aap.2011.07.005

Uman, L. S. (2011). Systematic reviews and meta-analysis. Journal of the Canadian Academy of Child and Adolescent Psychiatry, 20(1), 57-59.

Unsar, S., \& Sut, N. (2009). General assessment of the occupational accidents that occurred in Turkey between the years 2000 and 2005. Safety Science, 47, 614-619.

Van Der Schaaf, T., \& Kanse, L. (2004). Biases in incident reporting databases: An empirical study in the chemical process industry. Safety Science, 42(1), 57-67. doi: 10.1016/S0925-7535(03)00023-7

Van der Woning, Y. (2008). De invloed van kleur op het welzijn: Wit versus blauw. [The influence of color on well-being: White versus blue.] (Master's thesis, University of Twente, Enschede, The Netherlands). Retrieved from http://essay.utwente.nl/860/1/scriptie_van_der_Woning.pdf 
Van Dyck, C., Dimitrova, N. G., De Korne, D. F., \& Hiddema, F. (2013). Walk the talk: Leaders' enacted priority of safety, incident reporting, and error management. In Leading in health care organizations: Improving safety, satisfaction and financial performance (pp. 95-117). Emerald Group Publishing Limited.

Van Gemert, L. (2003). Het totstandkomen en functioneren van infectiepreventieprotocollen: Een onderzoek naar communicatie gestuurd door wet- en regelgeving (Doctoral dissertation). Retrieved from Pure Research Information University of Twente.

Vandenberg, R. J., Richardson, H. A., \& Eastman, L. J. (1999). The impact of high involvement work processes on organizational effectiveness - A secondorder latent variable approach. Group \& Organization Management, 24(3), 300-339.

Vassie, L. H., \& Lucas, W. R. (2001). An assessment of health and safety management within working groups in the UK manufacturing sector. Journal of Safety Research, 32, 479-490. doi: 10.1016/S00224375(01)00064-0

Vecchio-Sadus, A. M. (2007). Enhancing safety culture through effective communication. Safety Science Monitor, 11(3), 1-9.

Veiligheid bij werk aan spoor verbetert nauwelijks. (2017, September 5). Retrieved from https://www.ilent.nl/actueel/nieuws/2017/09/05/ veiligheid-bij-werk-aan-spoor-verbetert-nauwelijks/

Villanueva, V., \& Garcia, A. M. (2011). Individual and occupational factors related to fatal occupational injuries: A case-control study. Accident Analysis \& Prevention, 43(1), 123-127. doi: 10.1016/j.aap.2010.08.001

Vinodkumar, M. N., \& Bhasi, M. (2010). Safety management practices and safety behaviour: Assessing the mediating role of safety knowledge and motivation. Accident Analysis and Prevention, 42, 2082-2093. doi: 10.1016/j.aap.2010.06.021

Von der Heyde, A., Brandhorst, S., \& Kluge, A. (2015). The impact of safety audit timing and framing of the production outcomes on safety-related rule violations in a simulated production environment. Safety Science, 77, 205213. doi: 10.1016/j.ssci.2015.03.021 
Von Hippel, C., Magnum, S. L., Greenberger, D. B., Heneman, R. L., \& Skoglind, J. D. (1997). Temporary employment: Can organizations and employees both win? Academy of Management Executive, 11(1), 93-104.

Votano, S., \& Sunindijo, R. Y. (2014). Client safety roles in small and medium construction projects in Australia. Journal of Construction Engineering and Management, 140(9), 04014045. doi: 10.1061/(ASCE)C0.19437862.0000899

Vredenburgh, A. G. (2002). Organizational safety: Which management practices are most effective in reducing employee injury rates? Journal of Safety Research, 33(2), 259-276. doi: 10.1016/S0022-4375(02)00016-6

Wachter, J. K., \& Yorio, P. L. (2014). A system of safety management practices and worker engagement for reducing and preventing accidents: An empirical and theoretical investigation. Accident Analysis \& Prevention, 68, 117-130. doi: 10.1016/j.aap.2013.07.029

Wagstaff, A. S., \& Lie, J. S. (2011). Shift and night work and long working hours - A systematic review of safety implications. Scandinavian Journal of Work, Environment \& Health, 37(3), 173-185. doi: 10.5271/sjweh.3146

Waldorff, S. B., Reay, T., \& Goodrick, E. (2013). A tale of two countries: How different constellations of logics impact action. In Institutional logics in Action, Part A (pp. 99-129). Emerald Group Publishing Limited.

Wallace, J. C., Popp, E., \& Mondore, S. (2006). Safety climate as a mediator between foundation climates and occupational accidents: A grouplevel investigation. Journal of Applied Psychology, 9(3), 681-688. doi: 10.1037/0021-9010.91.3.681

Westaby, J. D., \& Lee, B. C. (2003). Antecedents of injury among youth in agricultural settings: A longitudinal examination of safety consciousness, dangerous risk taking, and safety knowledge. Journal of Safety Research, 34(3), 227-240. doi: 10.1016/S0022-4375(03)00030-6

Whu, T., Chen, C., \& Li, C. (2008). A correlation among safety leadership, safety climate and safety performance. Journal of Loss Prevention in the Process Industries, 21, 307-318. 
Williamson, A. M., Feyer, A., Cairns, D., \& Biancotti, D. (1997). The development of a measure of safety climate: The role of safety perceptions and attitudes. Safety Science, 25(1-3), 15-27.

Yule, S. (2003). Senior Management Influence on safety performance in the UK and US energy sectors. (Doctoral dissertation, University of Aberdeen, United Kingdom). Retrieved from http://efcog.org/wg/ism_pmi/docs/ Safety_Culture/Feb08/safety_culture_and_safety_climate_a_review_of_the_ literature.pdf

Zhou, Q., Fang, D., \& Wang, X. (2008). A method to identify strategies for the improvement of human safety behavior by considering safety climate and personal experience. Safety Science, 46, 1406-1419.

Zimmerman, B. J., \& Kitsantas, A. (2005). Homework practices and academic achievement: The mediating role of self-efficacy and perceived responsibility beliefs. Contemporary Educational Psychology, 30(4), 397417. doi: 10.1016/j.cedpsych.2005.05.003

Zohar, D. (1980). Safety climate in industrial organizations: Theoretical and applied implications. Journal of Applied Psychology, 65(1), 96-102. doi: 10.1037/0021-9010.65.1.96

Zohar, D. (2000). A group-level model of safety climate: Testing the effect of group climate on microaccidents in manufacturing jobs. Journal of Applied Psychology, 85(4), 587-596. doi: 10.1037/0021-9010.85.4.587

Zohar, D. (2002). The effects of leadership dimensions, safety climate, and assigned priorities on minor injuries in work groups. Journal of Organizational Behavior, 23(1), 75-92. doi: 10.1002/job.130

Zohar, D. (2010). Thirty years of safety climate research: Reflections and future directions. Accident Analysis \& Prevention, 42(5), 1517-1522. doi:10.1016/j.aap.2009.12.019

Zohar, D., \& Luria, G. (2003). The use of supervisory practices as leverage to improve safety behavior: A cross-level intervention model. Journal of Safety Research, 34(5), 567-577. doi: 10.1016/j.jsr.2003.05.006

Zwetsloot, G. I., Aaltonen, M., Wybo, J. L., Saari, J., Kines, P., \& De Beeck, R. O. (2013). The case for research into the zero accident vision. Safety Science, 58, 41-48. doi: 10.1016/j.ssci.2013.01.026 



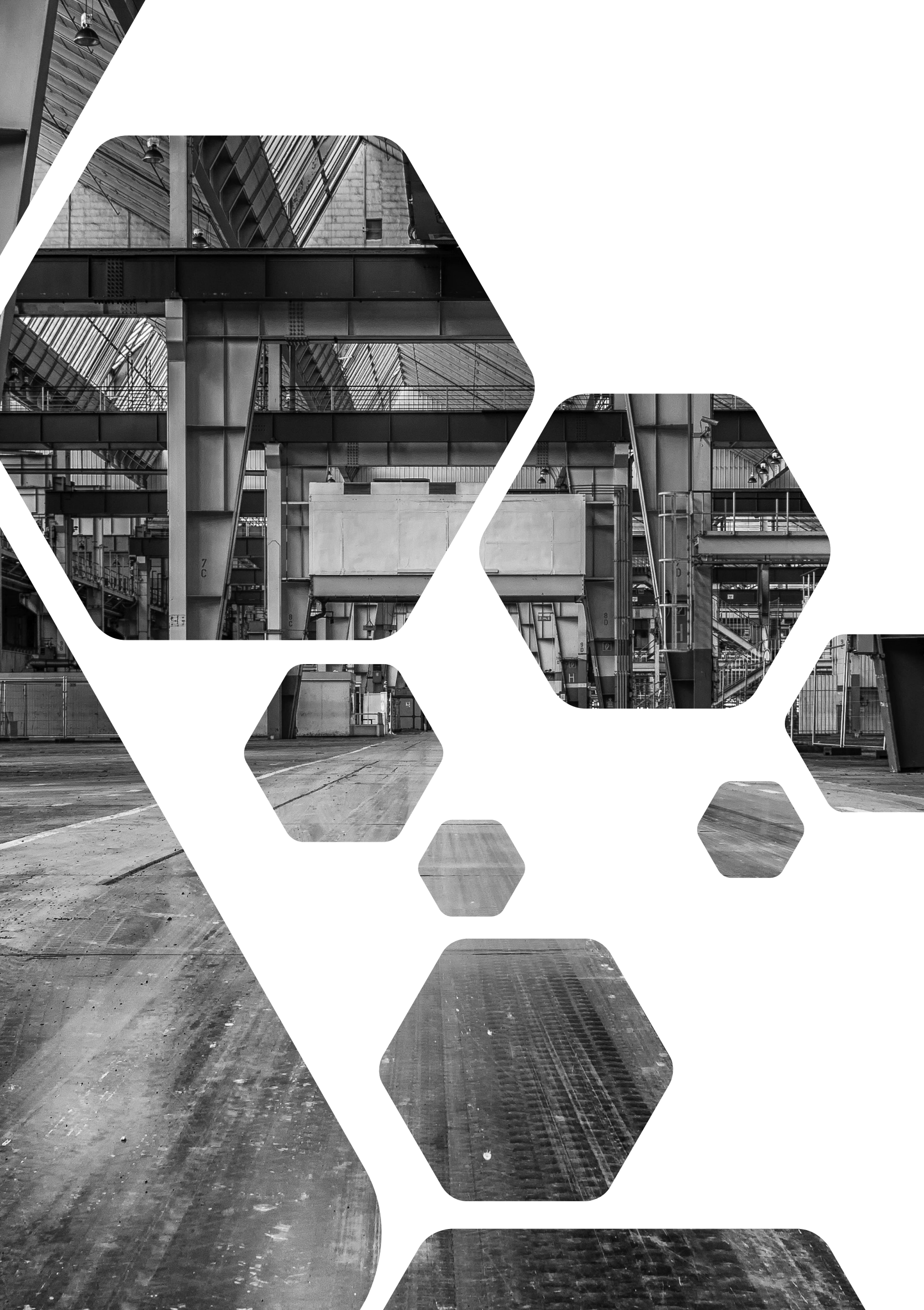


Appendices 


\section{Appendix A}

Reference materials as used in Chapter 2

Reference

Corresponding

table(s)

Aksorn, T., \& Hadikusumo, B. H. W. (2008). Critical success factors influencing safety program performance in Thai construction projects. Safety Science, 46(4), 709-727. doi: 10.1016/j. ssci.2007.06.006

Ali, H., Abdullah, N. A. C., \& Subramaniam, C. (2009).

Management practice in safety culture and its influence on workplace injury: An industrial study in Malaysia.

Disaster Prevention and Management, 18(5), 470-477. doi: 10.1108/09653560911003660

Allahyari, T., Rangi, N. H., Khalkhali, H., \& Khosravi, Y. (2014).

Occupational cognitive failures and safety performance in the workplace. International Journal of Occupational Safety and Ergonomics: JOSE, 20(1), 175-180.

Al-Refaie, A. (2013). Factors affect companies' safety performance in Jordan using structural equation modeling. Safety Science, 57, 169-178. doi:10.1016/j.ssci.2013.02.010

Alsamadani, R., Hallowell, M. R., Javernick-Will, A., \& Cabello, J. (2013). Relationships among language proficiency, communication patterns, and safety performance in small work crews in the United States. Journal of Construction Engineering and Management, 139(9), 1125-1134. doi: 10.1061/(ASCE) C0.1943-7862.0000724

Alsamadani, R., Hallowell, M., \& Javernick-Will, A. N. (2013). Measuring and modelling safety communication in small work crews in the US using social network analysis. Construction Management and Economics, 31(6), 568-579. doi:10.1080/014 46193.2012.685486

Amick III, B. C., Habeck, R. V., Hunt, A., Fossel, A. H., Chapin, A., Keller, R. B., \& Katz, J. N. (2000). Measuring the impact of organizational behaviors on work disability prevention and $2.2,2.4,2.5$ management. Journal of Occupational Rehabilitation, 10(1), 2138. doi: 10.1023/A:1009437728024 
Asfaw, A., \& Pana-Cryan, R. (2009). The impact of self-insuring for workers' compensation on the incidence rates of worker injury and illness. Journal of Occupational and Environmental Medicine, 51(12), 1466-1473. doi: 10.1097/ JOM.0b013e3181c16373

Austin, J., Kessler, M. L., Riccobono, J. E., \& Bailey, J. S. (1996). Using feedback and reinforcement to improve the performance and safety of a roofing crew. Journal of Organizational Behavior Management, 16(2), 49-75. doi: 10.1300/J075v16n02_04

Basha, S. A., \& Maiti, J. (2013). Relationships of demographic factors, job risk perception and work injury in a steel plant in India. Safety Science, 51(1), 374-381. doi: 10.1016/j. ssci.2012.08.005

Behm, M., \& Schneller, A. (2013). Application of the Loughborough Construction Accident Causation model: A framework for organizational learning. Construction Management \& Economics, 31(6), 580-595. doi: 10.1080/01446193.2012.690884

Bellamy, L. J. (2014). Exploring the relationship between major hazard, fatal and non-fatal accidents through outcomes and causes. Safety Science, 71, 93-103. doi: 10.1016/j. ssci.2014.02.009

Bjerkan, A. M. (2010). Health, environment, safety culture and climate - Analysing the relationships to occupational accidents. Journal of Risk Research, 13(4), 445-477. doi: 10.1080/13669870903346386

Boone, J., van Ours, J. C., Wuellrich, J. P., \& Zweimüller, J. (2011). Recessions are bad for workplace safety. Journal of Health Economics, 30(4), 764-773. doi: 10.1016/j.jhealeco.2011.05.013

$2.1,2.3,2.4$, 2.6

2.1, 2.3, 2.5, 2.7 assessment in petrochemical industry: A comparative study of two Algerian plants. Safety and Health at Work, 5(2), 60-65. doi: 10.1016/j.shaw.2014.03.005

Brondino, M., Silva, S. A., \& Pasini, M. (2012). Multilevel approach to organizational and group safety climate and safety performance: Co-workers as the missing link. Safety Science, 2.4 50(9), 1847-1856. doi: 10.1016/j.ssci.2012.04.010 
Brown, K. A., Willis, P. G., \& Prussia, G. E. (2000). Predicting safe employee behavior in the steel industry: Development and test $2.2,2.3,2.4$ of a sociotechnical model. Journal of Operations Management, 18(4), 445-465. doi: 10.1016/S0272-6963(00)00033-4

Bull, N., Riise, T., \& Moen, B. E. (2002). Work-related injuries and occupational health and safety factors in smaller enterprises A prospective study. Occupational Medicine, 52(2), 70-74. doi: 10.1093/occmed/52.2.70

Caponecchia, C., \& Sheils, I. (2011). Perceptions of personal vulnerability to workplace hazards in the Australian construction industry. Journal of Safety Research, 42(4), 253258. doi: 10.1016/j.jsr.2011.06.006

Cavazza, N., \& Serpe, A. (2009). Effects of safety climate on safety norm violations: Exploring the mediating role of attitudinal ambivalence toward personal protective equipment. Journal of $2.2,2.3,2.4$ 2.5 Safety Research, 40(4), 277-283. doi: 10.1016/j.jsr.2009.06.002

Chau, N., Bourgkard, E., Bhattacherjee, A., Ravaud, J. F., Choquet M., \& Mur, J. M. (2008). Associations of job, living conditions and lifestyle with occupational injury in working population: A population-based study. International Archives of Occupational and Environmental Health, 81(4), 379-389. doi: 10.1007/ s00420-007-0223-y

Chau, N., Gauchard, G. C., Siegfried, C., Benamghar, L., Dangelzer, J. L., Français, M., Jacquin, R., Sourdot, A., Perrin, P. P., \& Mur, J. M. (2004). Relationships of job, age, and life conditions with the causes and severity of occupational injuries in construction workers. International Archives of Occupational and Environmental Health, 77(1), 60-66. doi: 10.1007/s00420003-0460-7

Cheng, C. W., Leu, S. S., Lin, C. C., \& Fan, C. (2010). Characteristic analysis of occupational accidents at small construction enterprises. Safety Science, 48(6), 698-707. doi: 10.1016/j. ssci.2010.02.001

Chhokar, J. S., \& Wallin, J. A. (1984). A field study of the effect of feedback frequency on performance. Journal of Applied $2.1,2.3,2.6$ 2.6 Psychology, 69(3), 524-530. doi: 10.1037/0021-9010.69.3.524 
Chinander, K. R., Kleindorfer, P. R., \& Kunreuther, H. C. (1998). Compliance strategies and regulatory effectiveness of performance-based regulation of chemical accident risks. Risk Analysis, 18(2), 135-143. doi: 10.1111/j.1539-6924.1998. tb00925.x

Chinda, T., \& Mohamed, S. (2008). Structural equation model of construction safety culture. Engineering, Construction and Architectural Management, 15(2), 114-131. doi:

10.1108/09699980810852655

Choi, T. N. Y., Chan, D. W. M., \& Chan, A. P. C. (2012). Potential difficulties in applying the Pay for Safety Scheme (PFSS) in construction projects. Accident Analysis and Prevention, 48, 145-155. doi: 10.1016/j.aap.2011.04.015

Choudhry, R. (2012). Implementation of BBS and the impact of sitelevel commitment. Journal of Professional Issues in Engineering, Education and Practice, 138(4), 296-304. doi: 10.1061/(ASCE) EI.1943-5541.0000111.

Choudhry, R. M., \& Fang, D. (2008). Why operatives engage in unsafe work behavior: Investigating factors on construction sites. Safety Science, 46(4), 566-584. doi: 10.1016/j.ssci.2007.06.027

Cigularov, K. P., Chen, P. Y., \& Rosecrance, J. (2010). The effects of error management climate and safety communication on safety: A multi-level study. Accident Analysis and Prevention, $2.4,2.5$ 42(5), 1498-1506. doi: 10.1016/j.aap.2010.01.003

Clarke, S. (2006). Safety climate in an automobile manufacturing plant: The effects of work environment, job communication and safety attitudes on accidents and unsafe behavior. Personnel $2.2,2.3,2.5$, Review, 35(4), 413-430. doi: 10.1108/00483480610670580

Clarke, S., \& Ward, K. (2006). The role of leader influence tactics and safety climate in engaging employees' safety participation. Risk Analysis, 26(5), 1175-1185. doi: 10.1111/j.15396924.2006.00824.x 
Colley, S. K., Lincolne, J., \& Neal, A. (2013). An examination of the relationship amongst profiles of perceived organizational values, safety climate and safety outcomes. Safety Science, 51(1), 69-76. doi: 10.1016/j.ssci.2012.06.001

Collinson, D. L. (1999). 'Surviving the rigs': Safety and surveillance on North Sea oil installations. Organization Studies, 20(4), 579600. doi: 10.1177/0170840699204003

Conchie, S. M. (2013). Transformational leadership, intrinsic motivation, and trust: A moderated-mediated model of workplace safety. Journal of Occupational Health Psychology, 18(2), 198-210. doi: 10.1037/a0031805

Conchie, S. M., \& Burns, C. (2009). Improving occupational safety: Using a trusted information source to communicate about risk. Journal of Risk Research, 12(1), 13-25. doi: 10.1080/13669870802433749

Conchie, S. M., \& Donald, I. J. (2006). The role of distrust in offshore safety performance. Risk Analysis, 26(5), 1151-1159. doi: 10.1111/j.1539-6924.2006.00822.x

Conchie, S. M., Taylor, P. J., \& Donald, I. J. (2013). Promoting safety voice with safety-specific transformational leadership: The mediating role of two dimensions of trust. Journal of Occupational Health Psychology, 17(1), 105-115. doi: 10.1037/ a0025101

Cooper, M. D. (2006). Exploratory analyses of the effects of managerial support and feedback consequences on behavioral safety maintenance. Journal of Organizational Behavior Management, 26(3), 1-41. doi: 10.1300/J075v26n03_01

Cooper, M. D., \& Philips, R. A. (1994). Reducing accidents using goal setting and feedback: A field study. Journal of Occupational \& Organizational Psychology, 67(3), 219-240. doi: 10.1111/ j.2044-8325.1994.tb00564.x

Cooper, M. D., \& Philips, R. A. (2004). Exploratory analysis of the safety climate and safety behavior relationship. Journal of $2.1,2.4,2.6$ Safety Research, 35(5), 497-512. doi: 10.1016/j.jsr.2004.08.004 
Cree, T., \& Kelloway, E. K. (1997). Responses to occupational hazards: Exit and participation. Journal of Occupational Health Psychology, 2(4), 304-311. doi: 10.1037/1076-8998.2.4.304

De Koster, R. B. M., Stam, D., \& Balk, B. M. (2011). Accidents happen: The influence of safety-specific transformational leadership, safety consciousness, and hazard reducing systems on warehouse accidents. Journal of Operations Management, 29, 753-765. doi: 10.1016/j.jom.2011.06.005

De Souza Costa Neves Cavazotte, F., Pereira Duarte, C. J., \& Calvão Gobbo, A. M. (2013). Authentic leader, safe work: the influence of leadership on safety performance. Brazilian Business Review (English Edition), 10(2), 95-119. doi: 10.1108/LODJ-07-20110065

Dedobbeleer, N., \& German, P. (1987). Safety practices in construction industry. Journal of Occupational Medicine, 29(11), 863-868.

$2.1,2.3,2.5$, 2.6 $2.2,2.5,2.6$ for nehavior-based safety: A study of twenty industry-wide applications. Journal of Safety Research, 30(4), 237-249. doi: 10.1016/S0022-4375(99)00019-5

Didla, S., Mearns, K., \& Flin, R. (2009). Safety citizenship behaviour: A proactive approach to risk management. Journal of Risk Research, 12(3-4), 475-483. doi: 10.1080/13669870903041433

Dźwiarek, M. (2004). An analysis of accidents caused by improper functioning of machine control systems. International Journal of Occupational Safety and Ergonomics: JOSE, 10(2), 129-136. doi: 10.1080/10803548.2004.11076601

Elliott, K., \& Shelley, K. (2005). Impact of employee assistance programs on substance abusers and workplace safety. Journal of Employment Counseling, 42(3), 125-140. doi: 10.1002/ j.2161-1920.2005.tb00906.x

Elliott, K., \& Shelley, K. (2006). Effects of drugs and alcohol on behavior, job performance, and workplace safety. Journal of Employment Counseling, 43(3), 130-134. doi: 10.1002/j.21611920.2006.tb00012.x 
Fang, D. P., Chen, Y., \& Wong, L. (2006). Safety climate in construction industry: A case study in Hong Kong. Journal of Construction Engineering and Management-Asce, 132(6), 573584. doi: 10.1061/(ASCE)0733-9364(2006)132:6(573)

Fellner, D. J., \& Sulzer-Azaroff, B. (1985). Occupational safety: Assessing the impact of adding assigned or participative goalsetting. Journal of Organizational Behavior Management, 7(1), 3-24. doi: 10.1300/J075v07n01_02

Fernández-Muñiz, B., Montes-Peón, J. M., \& Vázquez-Ordás, C. J. (2007). Safety culture: Analysis of the causal relationships between its key dimensions. Journal of Safety Research, 38(6), 627-641. doi: 10.1016/j.jsr.2007.09.001

Fernández-Muñiz, B., Montes-Peón, J. M., \& Vázquez-Ordás, C. J. (2012). Safety climate in OHSAS 18001-certified organisations: Antecedents and consequences of safety behaviour. Accident Analysis and Prevention, 45, 745-758. doi: 10.1016/j. aap.2011.10.002

Filer, R. K., \& Golbe, D. L. (2003). Debt, operating margin, and investment in workplace safety. Journal of Industrial Economics, 51(3), 359-381. doi: 10.1111/1467-6451.00205

Ford, M. T., \& Wiggins, B. K. (2012). Occupational-level interactions between physical hazards and cognitive ability and skill requirements in predicting injury incidence rates. Journal of Occupational Health Psychology, 17(3), 268-278. doi: 10.1037/ a0028143

Fung, I. W. H., Lo, T. Y., \& Tung, K. C. F. (2012). Towards a better reliability of risk assessment: Development of a qualitative \& quantitative risk evaluation model $\left(Q^{2} \mathrm{REM}\right)$ for different trades of construction works in Hong Kong. Accident Analysis and Prevention, 48, 167-184. doi: 10.1016/j.aap.2011.05.011

Game, A. M. (2007). Workplace boredom coping: health, safety, and HR implications. Personnel Review, 36(5), 701-721. doi: 10.1108/00483480710774007 
Garcia, A. M., Boix, P., \& Canosa, C. (2004). Why do workers behave unsafely at work? Determinants of safe work practices in industrial workers. Occupational and Environmental Medicine, 61(3), 239-246. doi: 10.1136/oem.2002.005629

Ghosh, S. (2014). Does formal daily huddle meetings improve safety awareness? International Journal of Construction Education and Research, 10(4), 285-299. doi: 10.1080/15578771.2014.886642

Gittleman, J. L., Gardner, P. C., Haile, E., Sampson, J. M., Cigularov, K. P., Ermann, E. D., Stafford, P., \& Chen, P. Y. (2010). [Case Study] CityCenter and Cosmopolitan Construction Projects, Las Vegas, Nevada: Lessons learned from the use of multiple sources and

$2.3,2.4,2.5$, 2.6

2.5

$2.3,2.4,2.5$ mixed methods in a safety needs assessment. Journal of Safety Research, 41(3), 263-281. doi: 10.1016/j.jsr.2010.04.004

Goldenhar, L. M., Williams, L. J., \& Swanson, N. G. (2003). Modelling relationships between job stressors and injury and near-miss outcomes for construction labourers. Work \& Stress, 17(3), 218240. doi: 10.1080/02678370310001616144

Granerud, R. L., \& Rocha, R. S. (2011). Organisational learning and continuous improvement of health and safety in certified manufacturers. Safety Science, 49(7), 1030-1039. doi: 10.1016/j.ssci.2011.01.009

Gray, W. B., \& Jones, C. A. (1991). Longitudinal patterns of compliance with occupational safety and health administration health and safety regulations in the manufacturing sector. Journal of Human Resources, 26(4), 623-653. doi: $10.2307 / 145978$

Gressgård, L. J. (2014). Knowledge management and safety compliance in a high-risk distributed organizational system. Safety and Health at Work, 5(2), 53-59. doi: 10.1016/j. shaw.2014.03.002

Griffin, M. A., \& Hu, X. (2013). How leaders differentially motivate safety compliance and safety participation: The role of monitoring, inspiring, and learning. Safety Science, 60, 196-202. doi: 10.1016/j.ssci.2013.07.019 
Griffin, M. A., \& Neal, A. (2000). Perceptions of safety at work: A framework for linking safety climate to safety performance, knowledge, and motivation. Journal of Occupational Health Psychology, 5(3), 347-358. doi: 10.1037/1076-8998.5.3.347

Grunberg, L., Moore, S., \& Greenberg, E. (1996). The relationship of employee ownership and participation to workplace safety. Ergonomic and Industrial Democracy, 17(2), 221-241. doi: 10.1177/0143831X96172004

Gun, R. T. (1993). The role of regulations in the prevention of occupational injury. Safety Science, 16(1), 47-66. doi: 10.1016/0925-7535(93)90006-Y

Gyekye, S. A. (2006). Workers' perceptions of workplace safety: An African perspective. International Journal of Occupational Safety and Ergonomics: JOSE, 12(1), 31-42. doi: 10.1080/10803548.2006.11076667

Gyekye, S. A., \& Haybatollahi, M. (2014). Relationship between organizational justice and organizational safety climate: do fairness perceptions influence employee safety behaviour? International Journal of Occupational Safety and Ergonomics: JOSE, 20(2), 199-211. doi: 10.1080/10803548.2014.11077045

Gyekye, S. A., \& Salminen, S. (2007). Workplace safety perceptions and perceived organizational support: do supportive perceptions influence safety perceptions? International Journal of Occupational Safety and Ergonomics: JOSE, 13(2), 189-200. doi: 10.1080/10803548.2007.11076721

Gyekye, S. A., \& Salminen, S. (2009). Age and workers' perceptions of workplace safety: A comparative study. International Journal of Aging and Human Development, 68(2), 171-184. doi: 10.2190/AG.68.2.d

Gyekye, S. A., \& Salminen, S. (2009). Educational status and organizational safety climate: Does educational attainment influence workers' perceptions of workplace safety? Safety Science, 47(1), 20-28. doi: 10.1016/j.ssci.2007.12.007 
Gyekye, S. A., \& Salminen, S. (2010). Organizational safety climate and work experience. International Journal of Occupational Safety and Ergonomics: JOSE, 16(4), 431-443. doi: 10.1080/10803548.2010.11076856

Hadjimanolis, A., \& Boustras, G. (2013). Health and safety policies and work attitudes in Cypriot companies. Safety Science, 52, 50 56. doi: 10.1016/j.ssci.2012.03.012

$2.1,2.3,2.4$, $2.5,2.6$

Hajmohammad, S., \& Vachon, S. (2014). Safety culture: A catalyst for sustainable development. Journal of Business Ethics, 123(2), 263-281. doi: 10.1007/s10551-013-1813-0

Hallowell, M. R., \& Calhoun, M. E. (2011). Interrelationships among highly effective construction injury prevention strategies. Journal of Construction Engineering and Management, 137(11), 985-993. doi: 10.1061/(ASCE)C0.1943-7862.0000354

Hardison, D., Behm, M., Hallowell, M. R., \& Fonooni, H. (2014). Identifying construction supervisor competencies for effective site safety. Safety Science, 65, 45-53. doi: 10.1016/j. ssci.2013.12.013

Hare, B., \& Cameron, I. (2011). Site manager safety training. Engineering, Construction and Architectural Management, 18(6), 568-578. doi: 10.1108/09699981111180881

Haslam, C., Atkinson, S., Brown, S. S., \& Haslam, R. A. (2005). Anxiety and depression in the workplace: Effects on the individual and organisation. Journal of Affective Disorders, 88(2), 209-215. doi: 10.1016/j.jad.2005.07.009

Haviland, A., Burns, R., Gray, W., Ruder, T., \& Mendeloff, J. (2010). What kinds of injuries do OSHA inspections prevent? Journal of Safety Research, 41(4), 339-345. doi: 10.1016/j.jsr.2010.03.005

Hechanova-Alampay, R., \& Beehr, T. A. (2001). Empowerment, span of control, and safety performance in work teams after workforce reduction. Journal of Occupational Health Psychology, 6(4), 275-282. doi: 10.1037/1076-8998.6.4.275 
Hinze, J. (2002). Safety incentives: Do they reduce injuries? Practical Periodical on Structural Design and Construction, 7(2), $2.5,2.6$ 81-84. doi: 10.1061/(ASCE)1084-0680(2002)7:2(81)

Hoffmeister, K., Gibbons, A. M., Johnson, S. K., Cigularoy, K. P., Chen, P. Y., \& Rosecrance, J. C. (2014). The differential effects of transformational leadership facets on employee safety. Safety Science, 62, 68-78. doi:10.1016/j.ssci.2013.07.004

Holcroft, C. A., \& Punnett, L. (2009). Work environment risk factors for injuries in wood processing. Journal of Safety Research, 40(4), 247-255. doi: 10.1016/j.jsr.2009.05.001

Hon, C. K. H., Chan, A. P. C., \& Yam, M. C. H. (2014). Relationships between safety climate and safety performance of building repair, maintenance, minor alteration, and addition (RMAA) works. Safety Science, 65, 10-19. doi: 10.1016/j. ssci.2013.12.012

Hon, C. K. H., Hinze, J., \& Chan, A. P. C. (2014). Safety climate and injury occurrence of repair, maintenance, minor alteration and addition works. A comparison of workers, supervisors and managers. Facilities, 32(5-6), 188-207. doi: 10.1108/F-092011-0066

Hope, S., Øverland, S., Brun, W., \& Matthiesen, S. B. (2010). Associations between sleep, risk and safety climate: A study of offshore personnel on the Norwegian continental shelf. Safety Science, 48(4), 469-477. doi: 10.1016/j.ssci.2009.12.006

Huang, Y. H., Chen, P. Y., Krauss, A. D., \& Rogers, D. A. (2004). Quality of the execution of corporate safety policies and employee safety outcomes: Assessing the moderating role of supervisor safety support and the mediating role of employee safety

$2.2,2.3,2.5$, 2.6

\section{$2.2,2.3$} control. Journal of Business and Psychology, 18(4), 483-506. doi: 10.1023/B:JOBU.0000028448.01394.bf

Huang, Y. H., Ho, M., Smith, G. S., Chen, P. Y., \& Mith, S. (2006). Safety climate and self-reported injury: Assessing the mediating role of employee safety control. Accident Analysis and Prevention, 38(3), 425-433. doi: 10.1016/j.aap.2005.07.002 
Hystad, S. W., Bartone, P. T., \& Eid, J. (2014). Positive organizational behavior and safety in the offshore oil industry: Exploring the determinants of positive safety climate. Journal of Positive Psychology, 9(1), 42-53. doi: 10.1080/17439760.2013.831467

Jaselskis, E. J., \& Suazo, G. A. R. (1994). A survey of construction site safety in Honduras. Construction Management \& Economics, 12(3), 245-255. doi: 10.1080/01446199400000032

2.2, 2.3, 2.5, 2.6

$2.2,2.4,2.6$ knowledge/behavior and safety performance: Safety climate as a moderator in a multilevel study. Accident Analysis and Prevention, 42(5), 1468-1476. doi: 10.1016/j.aap.2009.08.017

Kamal, A. A. M., Sayed, G. M., Hassan, M. H., \& Massoud, A. A. (1988). Usage of personal protective devices among Egyptian industrial workers. American Journal of Industrial Medicine, 13(6), 707716. doi: 10.1002/ajim.4700130609

Kantermann, T., Haubruge, D., \& Skene, D. J. (2013). The shift-work accident rate is more related to the shift type than to shift rotation. Human and Ecological Risk Assessment, 19(6), 15861594. doi: 10.1080/10807039.2012.708263

Kaskutas, V., Dale, A. M., Lipscomb, H., Gaal, J., Fuchs, M., \& Evanoff, B. (2010). Fall prevention among apprentice carpenters. Scandinavian Journal of Work, Environment and Health, 36(3), 258-265. doi: 10.2307/40967854

Khosravi, Y., Asilian-Mahabadi, H., Hajizadeh, E., HassanzadehRangi, N., Bastani, H., Khavanin, A., \& Mortazavi, S. B. (2014). Modeling the factors affecting unsafe behavior in the construction industry from safety supervisors' perspective. Journal of Research in Health Sciences, 14(1), 29-35.

Koh, T. Y., \& Rowlinson, S. (2012). Relational approach in managing construction project safety: A social capital perspective. Accident Analysis and Prevention, 48, 134-144. doi: 10.1016/j. aap.2011.03.020 
Komaki, J. L., Collins, R. L., \& Penn, P. (1982). The role of performance antecedents and consequences in work motivation. Journal of Applied Psychology, 67(3), 334-340. doi: 10.1037/0021-9010.67.3.334

Lee, C. (2011). Exploring the characteristics of organizational factors on safety climate in Taiwan. Applied Mechanics and Materials, 58-60, 662-667. doi: 10.4028/www.scientific.net/ AMM.58-60.662

Lee, K., Shon, D., \& Oah, S. (2014). The relative effects of global and specific feedback on safety behaviors. Journal of Organizational Behavior Management, 34(1), 16-28. doi: 10.1080/01608061.2013.878264

Lee, T. Z., Wu, C. H., \& Hong, C. W. (2007). An empirical investigation of the influence of safety climate on organizational citizenship behavior in Taiwan's facilities. International Journal of Occupational Safety and Ergonomics: JOSE, 13(3), 255-269. doi: 10.1080/10803548.2007.11076726

Leung, M. Y., Chan, Y. S., \& Yuen, K. W. (2010). Impacts of stressors and stress on the injury incidents of construction workers in Hong Kong. Journal of Construction Engineering and Management, 136(10), 1093-1103. doi: 10.1061/(ASCE) C0.1943-7862.0000216

Levin, L., Oler, J., \& Whiteside, J. R. (1985). Injury incidence rates in a paint company on rotating production shifts. Accident Analysis and Prevention, 17(1), 67-73.

Levine, D. I., \& Toffel, M. W. (2010). Quality management and job quality: How the ISO 9001 standard for quality management systems affects employees and employers. Management Science, 56(6), 978-996. doi: 10.2139/ssrn.1237730

Li, F., Jiang, L., Yao, X., \& Li, Y. (2013). Job demands, job resources and safety outcomes: The roles of emotional exhaustion and safety compliance. Accident Analysis and Prevention, 51, 243251. doi: 10.1016/j.aap.2012.11.029 
Liao, P. C., Lei, G., Fang, D., \& Liu, W. (2014). The relationship between communication and construction safety climate in China. KSCE Journal of Civil Engineering, 18(4), 887-897. doi: 10.1007/s12205-014-0492-4

Lindroos, O. (2009). Relationships between observed and perceived deviations from normative work procedures. Ergonomics, 52(12), 1487-1500. doi: 10.1080/00140130903197461

Lingard, H. (2001). The effect of first aid training on objective safety behaviour in Australian small business construction firms. Construction Management and Economics, 19(6), 611-618. doi: 10.1080/01446190110117617

Liu, H., Burns, R. M., Schaefer, A. G., Ruder, T., Nelson, C., Haviland, A. M., Gray, W. B., \& Mendeloff, J. (2010). The Pennsylvania certified safety committee program: An evaluation of $2.1,2.3$ participation and effects on work injury rates. American Journal of Industrial Medicine, 53(8), 780-791. doi: 10.1002/ajim.20861

Loudoun, R. J. (2010). Injuries sustained by young males in construction during day and night work. Construction Management and Economics, 28(12), 1313-1320. doi: 10.1080/01446193.2010.521760

Martin, H., \& Lewis, T. M. (2014). Pinpointing safety leadership factors for safe construction sites in Trinidad and Tobago. Journal of Construction Engineering and Management, 140, 04013046. doi: 10.1061/(ASCE)C0.1943-7862.0000795

Mearns, K. J., \& Reader, T. (2008). Organizational support and safety outcomes: An un-investigated relationship? Safety Science, 46(3), 388-397. doi: 10.1016/j.ssci.2007.05.002

Mearns, K., Hope, L., Ford, M. T., \& Tetrick, L. E. (2010). Investment in workforce health: Exploring the implications for workforce safety climate and commitment. Accident Analysis and Prevention, 42(5), 1445-1454. doi: 10.1016/j.aap.2009.08.009 
Michael, J. H., Guo, Z. G., Wiedenbeck, J. K., \& Ray, C. D. (2006). Production supervisor impacts on subordinates' safety outcomes: An investigation of leader-member exchange and safety communication. Journal of Safety Research, 37(5), 469477. doi: 10.1016/j.jsr.2006.06.004

Mikkelsen, A., Ringstad, A. J., \& Steineke, J. M. (2004). Working time arrangements and safety for offshore workers in the North Sea. Safety Science, 42(3), 167-184. doi: 10.1016/S09257535(03)00025-0

Mitropoulos, P., \& Cupido, G. (2009). Safety as an emergent property: Investigation into the work practices of highreliability framing crews. Journal of Construction Engineering \& Management, 135(5), 407-415. doi: 10.1061/(ASCE)C0.19437862.0000002

Morse, T., Bracker, A., Warren, N., Goyzueta, J., \& Cook, M. (2013). Characteristics of effective health and safety committees: Survey results. American Journal of Industrial Medicine, 56(2), 163-179. doi: 10.1002/ajim.22096

Neitzel, R. L., Seixas, N. S., Harris, M. J., \& Camp, J. (2008). Exposure to fall hazards and safety climate in the aircraft maintenance industry. Journal of Safety Research, 39(4), 391-402. doi: 10.1016/j.jsr.2008.02.033

Nelson, D. I., \& Canty, G. A. (1993). Safety and health provisions of LIST contractors and state programs. Applied Occupational and Environmental Hygiene, 8(10), 859-865. doi: 10.1080/1047322X.1993.10388215

Nenonen, S., \& Vasara, J. (2013). Safety management in multiemployer worksites in the manufacturing industry: Opinions on co-operation and problems encountered. International Journal of Occupational Safety and Ergonomics: $2.3,2.4,2.5$, 2.6 JOSE, 19(2), 167-183. doi: 10.1080/10803548.2013.11076976

Nesheim, T., \& Gressgård, L. J. (2014). Knowledge sharing in a complex organization: Antecedents and safety effects. Safety Science, 62, 28-36. doi: 10.1016/j.ssci.2013.07.018 
Niskanen, T. (2012). Results of Finnish national survey in the chemical industry on EU legislation concerned with risk assessment and safety compliance climate. Journal of Loss

Prevention in the Process Industries, 25(3), 535-543. doi: 10.1016/j.jlp.2011.12.010

Niskanen, T., Louhelainen, K., \& Hirvonen, M. L. (2014). An evaluation of the effects of the occupational safety and health inspectors' supervision in workplaces. Accident Analysis and Prevention, 68, 139-155. doi: 10.1016/j.aap.2013.11.013

Niskanen, T., Naumanen, P., \& Hirvonen, M. L. (2012). Safety compliance climate concerning risk assessment and preventive measures in EU legislation: A Finnish survey. Safety Science, 50(9), 1929-1937. doi: 10.1016/j.ssci.2012.05.005

Nöhammer, E., Schusterschitz, C., \& Stummer, H. (2010). Determinants of employee participation in workplace health promotion. International Journal of Workplace Health Management, 3(2), 97-110. doi: 10.1108/17538351011055005

Pagell, M., Dibrell, C., Veltri, A., \& Maxwell, E. (2014). Is an efficacious operation a safe operation: The role of operational practices in worker safety outcomes. IEEE Transactions on Engineering Management, 61(3), 511-521. doi: 10.1109/ TEM.2014.2316249

Parboteeah, K., \& Kapp, E. (2008). Ethical climates and workplace safety behaviors: An empirical investigation. Journal of Business Ethics, 80(3), 515-529. doi: 10.1007/s10551-007-9452-y

Parkes, K. R. (2003). Shiftwork and environment as interactive predictors of work perceptions. Journal of Occupational Health Psychology, 8(4), 266-281. doi: 10.1037/1076-8998.8.4.266

Pettersson-Strömbäck, A., Liljelind, I., Neely, G., \& Järvholm, B. (2008). Workers' interpretation of self-assessment of exposure. Annals of Occupational Hygiene, 52(7), 663-671. doi: 10.1093/ annhyg/men 042

Philips, J. A., \& Brown, K. C. (1992). Industrial workers on a rotating shift pattern: Adaptation and injury status. American Association of Occupational Health Nurses Journal, 40(10), 468476. 
Pollack, K. M., Agnew, J., Slade, M. D., Cantley, L., Taiwo, O., Vegso, S., Sircar, K. \& Cullen, M. R. (2007). Use of employer administrative databases to identify systematic causes of injury in aluminum manufacturing. American Journal of Industrial Medicine, 50(9), 676-686. doi: 10.1002/ajim.20493

Powell, R., \& Copping, A. (2010). Sleep deprivation and its consequences in construction workers. Journal of Construction Engineering and Management, 136(10), 1086-1092. doi: 10.1061/(ASCE)C0.1943-7862.0000211

Probst, T. M. (2004). Safety and insecurity: Exploring the moderating effect of organizational safety climate. Journal of Occupational Health Psychology, 9(1), 3-10. doi: 10.1037/10768998.9.1.3

Prussia, G. E., Brown, K. A., \& Willis, P. G. (2003). Mental models of safety: Do managers and employees see eye to eye? Journal of Safety Research, 34(2), 143-156. doi: 10.1016/S0022$2.2,2.3,2.4$ 4375(03)00011-2

Reber, R. A, \& Wallin, J. A. (1984). The effects of training, goal setting, and knowledge of results on safe behavior: A component analysis. Academy of Management Journal, 27(3), 544-560. doi: $10.2307 / 256044$

Robinson, J. C., \& Shor, G. M. (1989). Business-cycle influences on work-related disability in construction and manufacturing. Milbank Quarterly, 67, 92-113. doi: 10.2307/3350237

Sampson, J. M., DeArmond, S., \& Chen, P. Y. (2014). Role of safety stressors and social support on safety performance. Safety Science, 64, 137-145. doi: 10.1016/j.ssci.2013.11.025

Shannon, H. S., Walters, V., Lewchuk, W., Richardson, J., Moran, L. A., Haines, T., \& Verma D. (1996). Workplace organizational correlates of lost-time accident rates in manufacturing. American Journal of Industrial Medicine, 29(3), 258-268. doi: 10.1002/(SICI)1097-0274(199603)29:3<258::AIDAJIM5>3.0.CO;2-M 
Shi, L., Liu, Q., \& Wu, K. (2013). Relationships among safety manager behavior, job insecurity atmosphere, counterproductive work behavior and quality performance. Journal of Applied Sciences, 13(17), 3548-3552. doi: 10.3923/jas.2013.3548.3552

Simard, M., \& Marchand, A. (1997). Workgroups' propensity to comply with safety rules: The influence of micro-macro organisational factors. Ergonomics, 40(2), 172-188. doi: 10.1080/001401397188288

Sinclair, R. C., \& Cunningham, T. R. (2014). Safety activities in small businesses. Safety Science, 64, 32-38. doi: 10.1016/j. ssci.2013.11.022

2.2, 2.3, 2.5, $2.6,2.7$

$2.1,2.3,2.5$, 2.6

Siu, O. L., Phillips, D. R., \& Leung, T. W. (2003). Age differences in safety attitudes and safety performance in Hong Kong construction workers. Journal of Safety Research, 34(2), 199205. doi: 10.1016/S0022-4375(02)00072-5

Siu, O. L., Phillips, D. R., \& Leung, T. W. (2004). Safety climate and safety performance among construction workers in Hong Kong: The role of psychological strains as mediators. Accident Analysis and Prevention, 36(3), 359-366. doi: 10.1016/S00014575(03)00016-2

Smith, A. P. (2005). Caffeine at work. Human Psychopharmacology: Clinical and Experimental, 20(6), 441-445. doi: 10.1002/ hup.705

Sparer, E. H., Murphy, L. A., Taylor, K. M., \& Dennerlein, J. T. (2013). Correlation between safety climate and contractor safety assessment programs in construction. American Journal of Industrial Medicine, 56(12), 1463-1472. doi: 10.1002/ ajim. 22241

Suarez Sanchez, A., Riesgo Fernandez, P., Sanchez Lasheras, F., de Cos Juez, F. J., \& Garcia Nieto, P. J. (2011). Prediction of work-related accidents according to working conditions 2.1, 2.3, 2.5, using support vector machines. Applied Mathematics $2.6,2.7$ and Computation, 218(7), 3539-3552. doi: 10.1016/j. amc.2011.08.100 
Sulzer-Azaroff, B., Loafman, B., Merante, R. J., \& Hlavacek, A. C. (1990). Improving occupational safety in a large industrial plant. Journal of Organizational Behavior Management, 11(1), 99-120. doi: 10.1300/J075v11n01_07

Tam, C. M., \& Fung, I. W. H. (1998). Effectiveness of safety management strategies on safety performance in Hong Kong. Construction Management and Economics, 16(1), 49-55. doi: $10.1080 / 014461998372583$

Teo, E. A. L., \& Feng, Y. (2009). The role of safety climate in predicting safety culture on construction sites. Architectural Science Review, 52(1), 5-16. doi: 10.3763/asre.2008.0037

Tharaldsen, J. E., Mearns, K. J., \& Knudsen, K. (2010). Perspectives on safety: The impact of group membership, work factors and trust on safety performance in UK and Norwegian drilling $2.2,2.3,2.6$ company employees. Safety Science, 48(8), 1062-1072. doi: 10.1016/j.ssci.2009.06.003

Thompson, R. C., Hilton, T. F., \& Witt, L. A. (1998). Where the safety rubber meets the shop floor: A confirmatory model of management influence on workplace safety. Journal of Safety Research, 29(1), 15-24. doi: 10.1016/S0022-4375(97)00025-X

Törner, M., \& Pousette, A. (2009). Safety in construction - A comprehensive description of the characteristics of high safety standards in construction work, from the combined perspective of supervisors and experienced workers. Journal of Safety $2.3,2.4,2.5$ 2.6 Research, 40(6), 399-409. doi: 10.1016/j.jsr.2009.09.005

Torp, S., \& Grøgaard, J. B. (2009). The influence of individual and contextual work factors on workers' compliance with health and safety routines. Applied Ergonomics, 40(2), 185-193. doi: 10.1016/j.apergo.2008.04.002

Torp, S., \& Moen, B. E. (2006). The effects of occupational health and safety management on work environment and health: A prospective study. Applied Ergonomics, 37(6), 775-783. doi: 10.1016/j.apergo.2005.11.005

Torp, S., Riise, T., \& Moen, B. E. (2000). Systematic health, environment and safety activities: Do they influence occupational environment, behaviour and health? Occupational Medicine, 50(5), 326-333. doi: 10.1093/occmed/50.5.326 
Tucker, P., Barton, J., \& Folkard, S. (1996). Comparison of eight and 12 hour shifts: Impacts an health, wellbeing, and alertness during the shift. Occupational and Environmental Medicine, 53(11), 767-772. doi: 10.1136/oem.53.11.767

Vassie, L. H., \& Lucas, W. R. (2001). An assessment of health and safety management within working groups in the UK manufacturing sector. Journal of Safety Research, 32(4), 479490. doi: 10.1016/S0022-4375(01)00064-0

Vinodkumar, M. N., \& Bhasi, M. (2009). Safety climate factors and its relationship with accidents and personal attributes in the chemical industry. Safety Science, 47(5), 659-667. doi: $2.2,2.3,2.4$ 10.1016/j.ssci.2008.09.004

Vinodkumar, M. N., \& Bhasi, M. (2010). Safety management practices and safety behaviour: Assessing the mediating role of safety knowledge and motivation. Accident Analysis and Prevention, 42(6), 2082-2093. doi: 10.1016/j.aap.2010.06.021

Votano, S., \& Sunindijo, R. Y. (2014). Client safety roles in small and medium construction projects in Australia. Journal of Construction Engineering and Management, 140(9), 04014045. doi: 10.1061/(ASCE)C0.1943-7862.0000899

Wachter, J. K., \& Yorio, P. L. (2014). A system of safety management practices and worker engagement for reducing and preventing accidents: An empirical and theoretical investigation. Accident Analysis and Prevention, 68, 117-130. doi: 10.1016/j. aap.2013.07.029

Weil, D. (2001). Assessing OSHA performance: New evidence from the construction industry. Journal of Policy Analysis and Management, 20(4), 651-674. doi: 10.1002/pam.1022

Williamson, A. M., \& Feyer, A. M. (1995). Causes of accidents and the time of day. Work \& Stress, 9(2-3), 158-164. doi: 
Willis, P. G., Brown, K. A., \& Prussia, G. E. (2012). Does employee safety influence customer satisfaction? Evidence from the electric utility industry. Journal of Safety Research, 43, 389-396. doi: 10.1016/j.jsr.2012.10.003

Wirtz, A., Nachreiner, F., \& Rolfes, K. (2011). Working on Sundays - Effects on safety, health, and work-life balance. Chronobiology International, 28(4), 361-370. doi: 10.3109/07420528.2011.565896

Yung, P. (2009). Institutional arrangements and construction safety in China: An empirical examination. Construction Management and Economics, 27(5), 439-450. doi: $10.1080 / 01446190902855633$

Zacharatos, A., Barling, J., \& Iverson, R. D. (2005). High-performance work systems and occupational safety. Journal of Applied Psychology, 90(1), 77-93. doi: 10.1037/0021-9010.90.1.77

Zhou, Q., Fang, D., \& Mohamed, S. (2011). Safety climate improvement: Case study in a Chinese construction company. $2.3,2.5,2.6$, Journal of Construction Engineering and Management, 137(1), 86-95. doi: 10.1061/(ASCE)C0.1943-7862.0000241

Zohar, D. (2000). A group-level model of safety climate: Testing the effect of group climate on microaccidents in manufacturing jobs. Journal of Applied Psychology, 85(4), 587-596. doi: 10.1037/0021-9010.85.4.587

Zwetsloot, G. I. J. M., Drupsteen, L., \& De Vroome, E. M. M. (2014). Safety, reliability and worker satisfaction during organizational change. Journal of Loss Prevention in the Process Industries, 27, 1-7. doi: 10.1016/j.jlp.2013.10.008 



\section{Appendix B}

Dutch and English items from Chapter 6

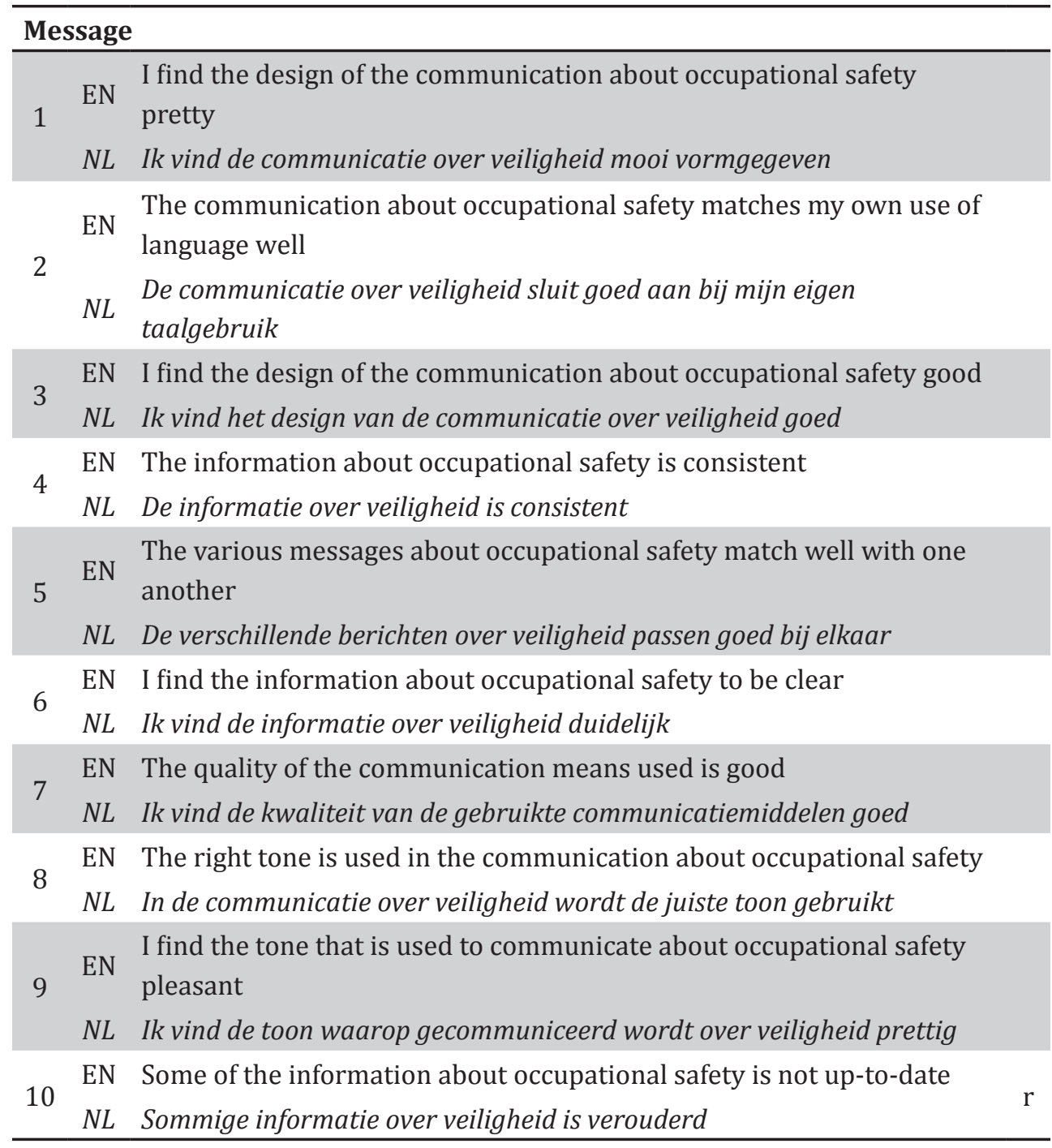

Note: $\mathrm{r}=$ recoded item 


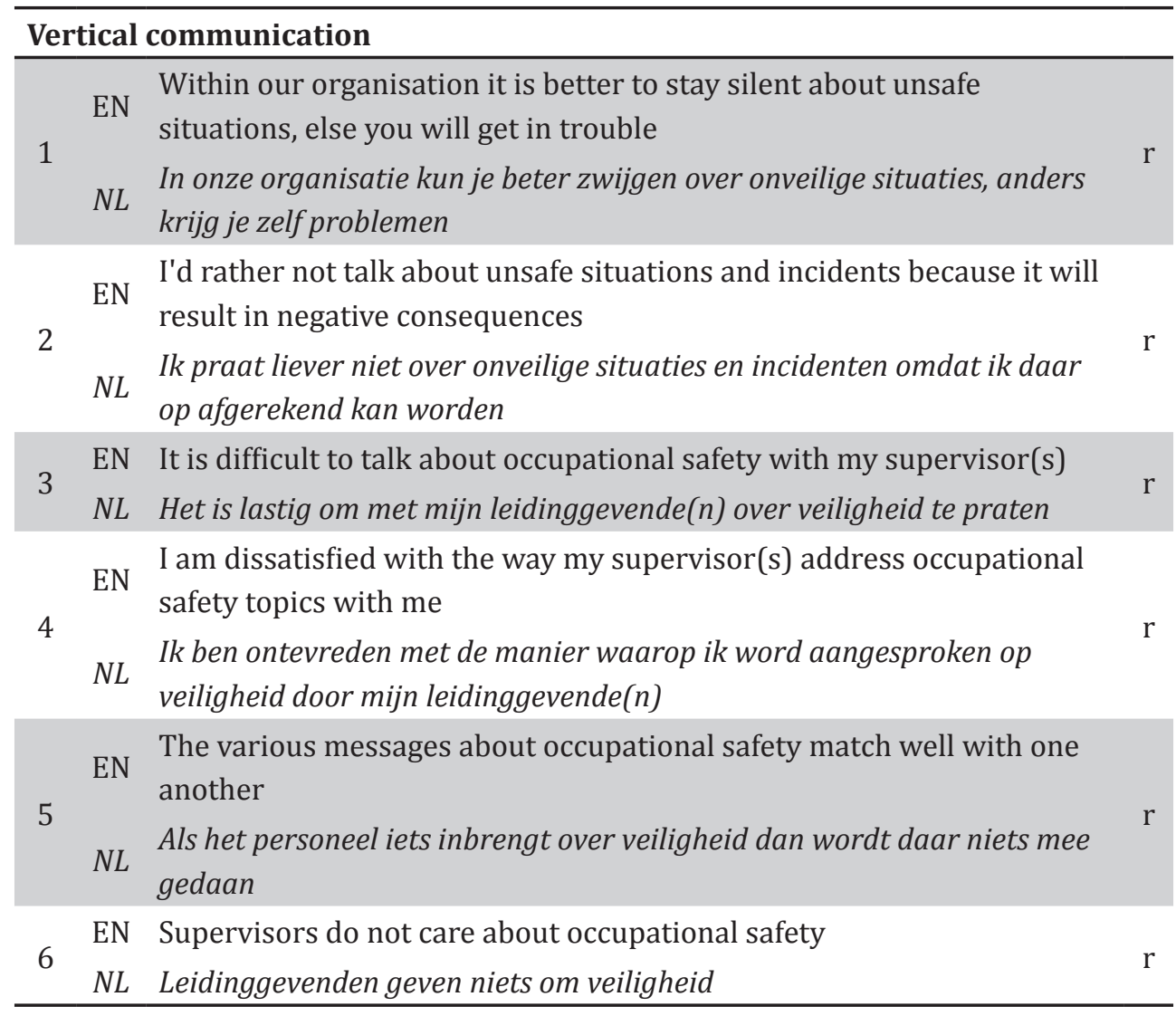

Note: $\mathrm{r}=$ recoded item 


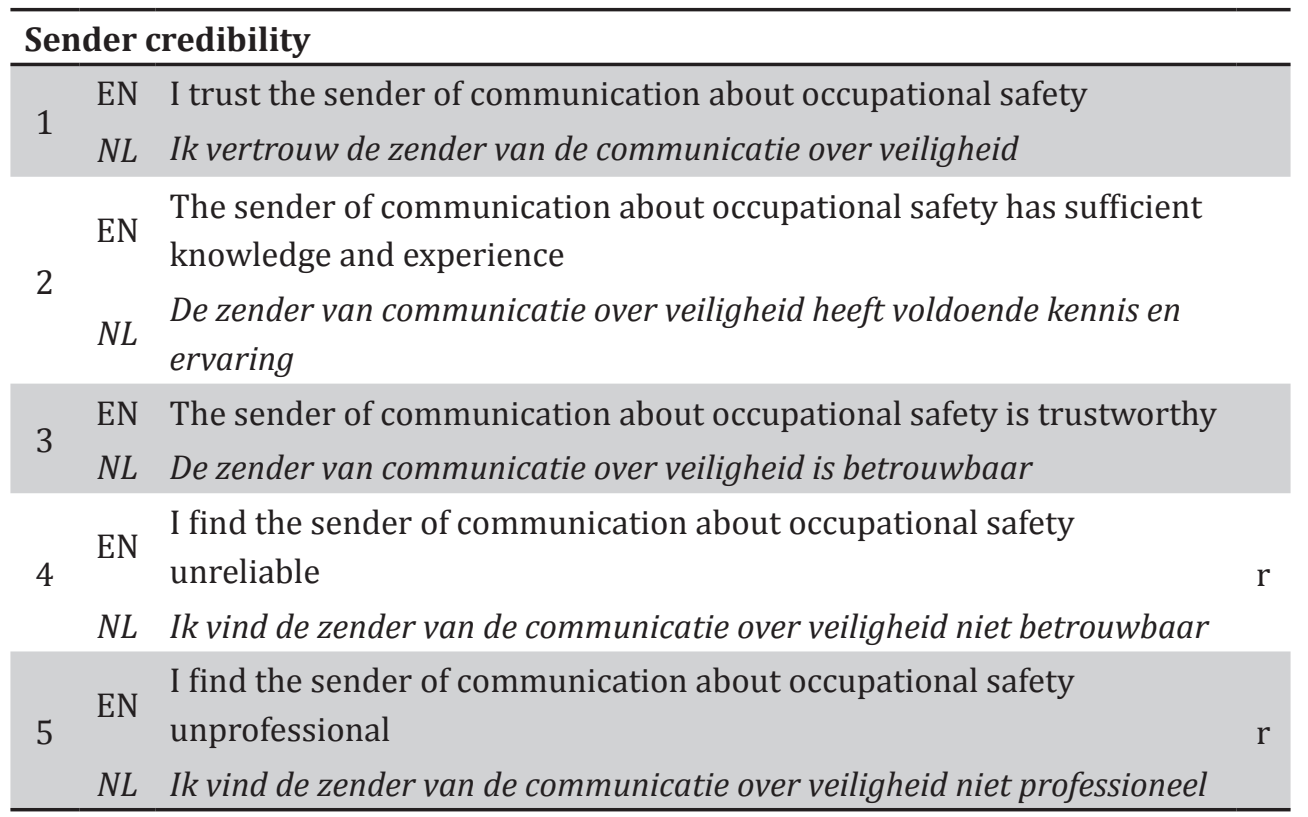

Note: $\mathrm{r}=$ recoded item

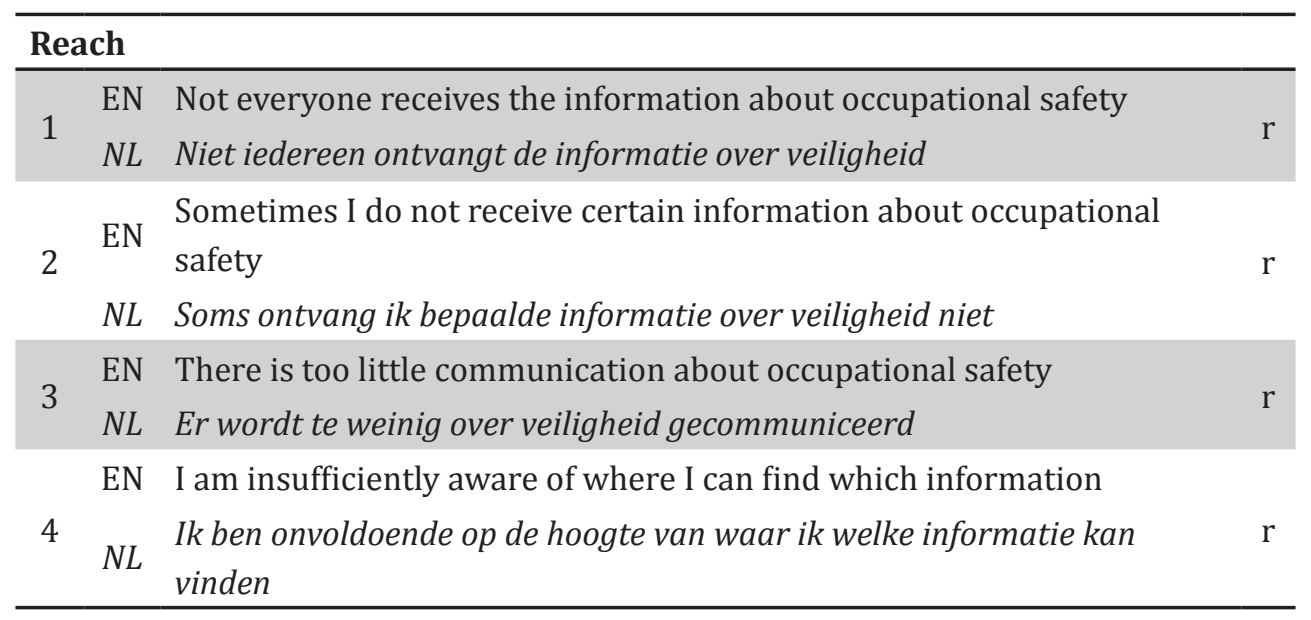

Note: $\mathrm{r}=$ recoded item 


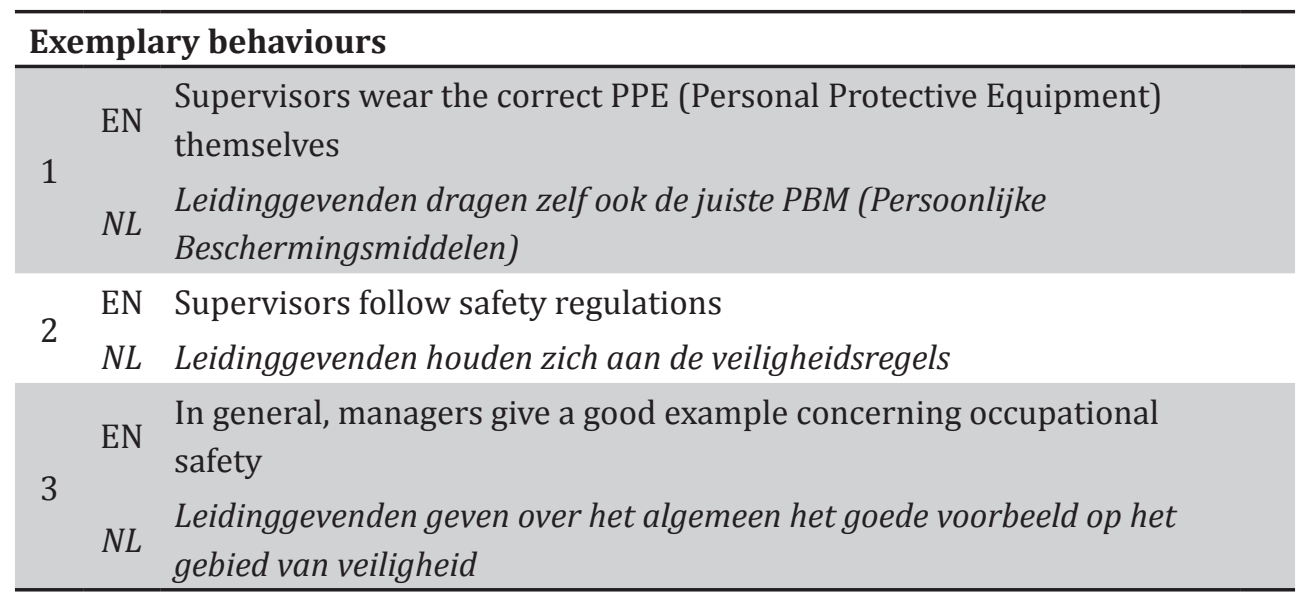

\begin{tabular}{|c|c|c|}
\hline \multicolumn{3}{|c|}{ Timing } \\
\hline \multirow[t]{2}{*}{1} & EN & $\begin{array}{l}\text { There is a good balance between the quantity of information about } \\
\text { occupational safety that I receive via my colleagues and that I hear via } \\
\text { my supervisor(s) }\end{array}$ \\
\hline & $N L$ & $\begin{array}{l}\text { Er is een goede balans tussen de hoeveelheid informatie over veiligheid } \\
\text { die ik hoor via mijn collega's en die ik hoor via mijn leidinggevende(n) }\end{array}$ \\
\hline \multirow{2}{*}{2} & EN & I receive the information about occupational safety when I need it \\
\hline & $N L$ & Ik krijg de informatie over veiligheid wanneer ik die nodig heb \\
\hline \multirow{2}{*}{3} & EN & Communication about occupational safety occurs at the right moments \\
\hline & $N L$ & Er wordt op de juiste momenten gecommuniceerd over veiligheid \\
\hline \multirow{2}{*}{4} & EN & $\begin{array}{l}\text { I am brought up to speed about changes in the area of occupational } \\
\text { safety }\end{array}$ \\
\hline & $N L$ & $\begin{array}{l}\text { Ik word op de hoogte gebracht van veranderingen op het gebied van } \\
\text { veiligheid }\end{array}$ \\
\hline
\end{tabular}




\begin{tabular}{lll}
\hline \multicolumn{1}{l}{ Match with practice } \\
\hline 1 & EN & $\begin{array}{l}\text { The information about occupational safety that I receive is relevant for } \\
\text { my function }\end{array}$ \\
& NL & De informatie over veiligheid die ik krijg, is relevant voor mijn functie \\
2 & EN & $\begin{array}{l}\text { I receive information about occupational safety that is unnecessary for } \\
\text { my job }\end{array}$ \\
& NL & Ik krijg informatie over veiligheid die onnodig is voor mijn werk \\
3 & EN & $\begin{array}{l}\text { The information about occupational safety matches with my work } \\
\text { activities }\end{array}$ \\
NL & De informatie over veiligheid past bij de werkzaamheden die ik doe \\
4 & EN & I receive information about occupational safety that is not useful to me \\
NL & Ik krijg informatie over veiligheid waar ik niets mee kan
\end{tabular}

Note: $\mathrm{r}=$ recoded item

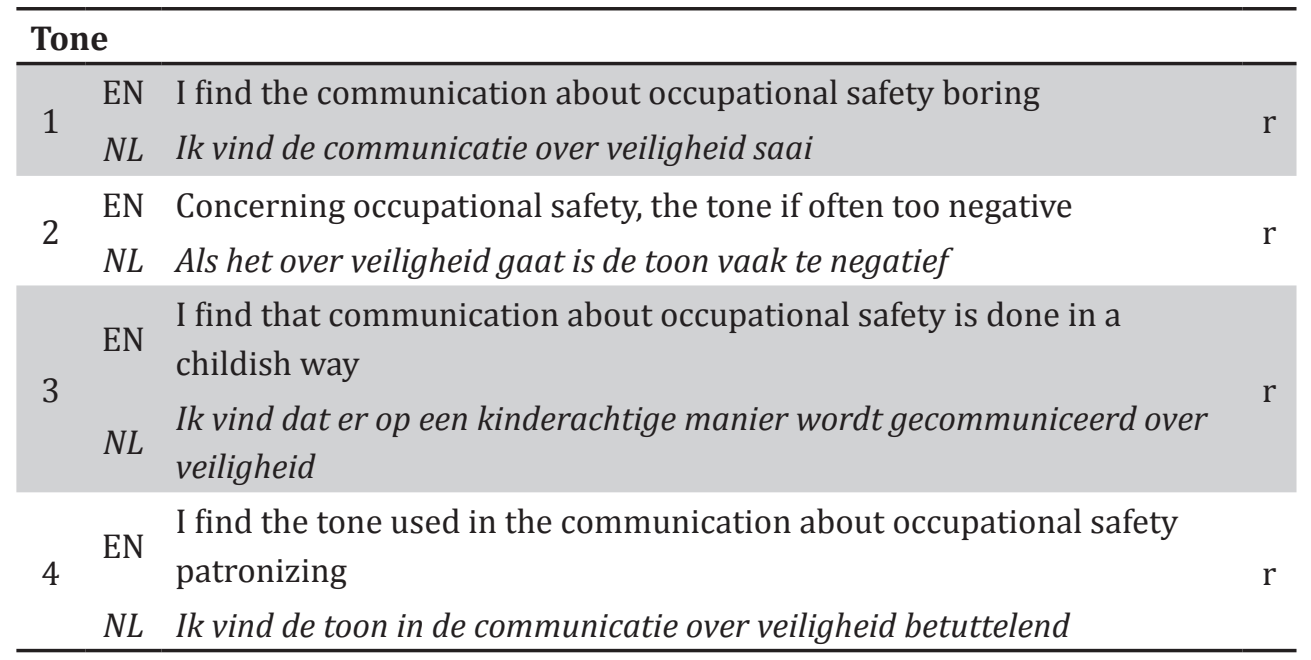

Note: $\mathrm{r}=$ recoded item 

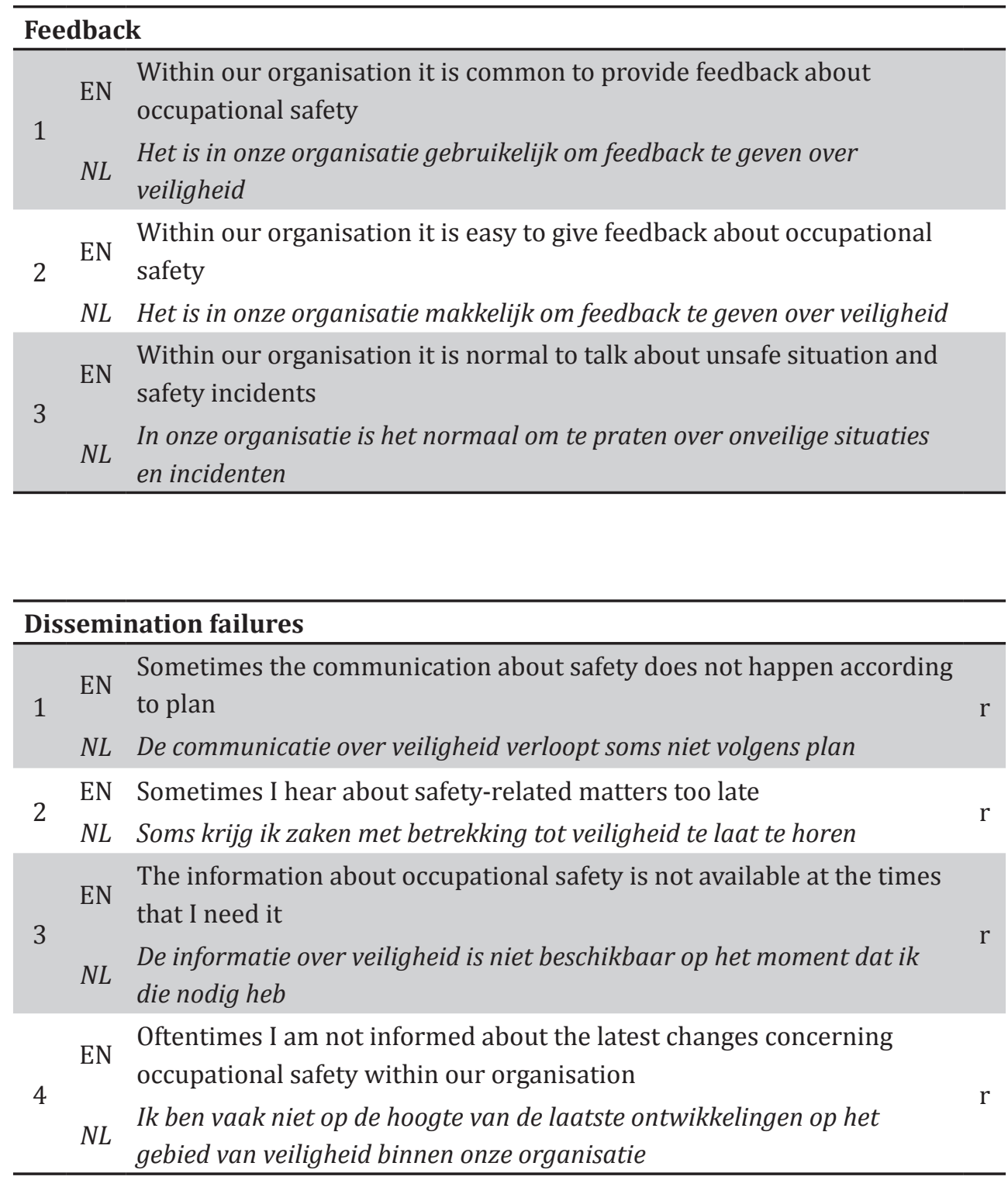

Note: $\mathrm{r}=$ recoded item 


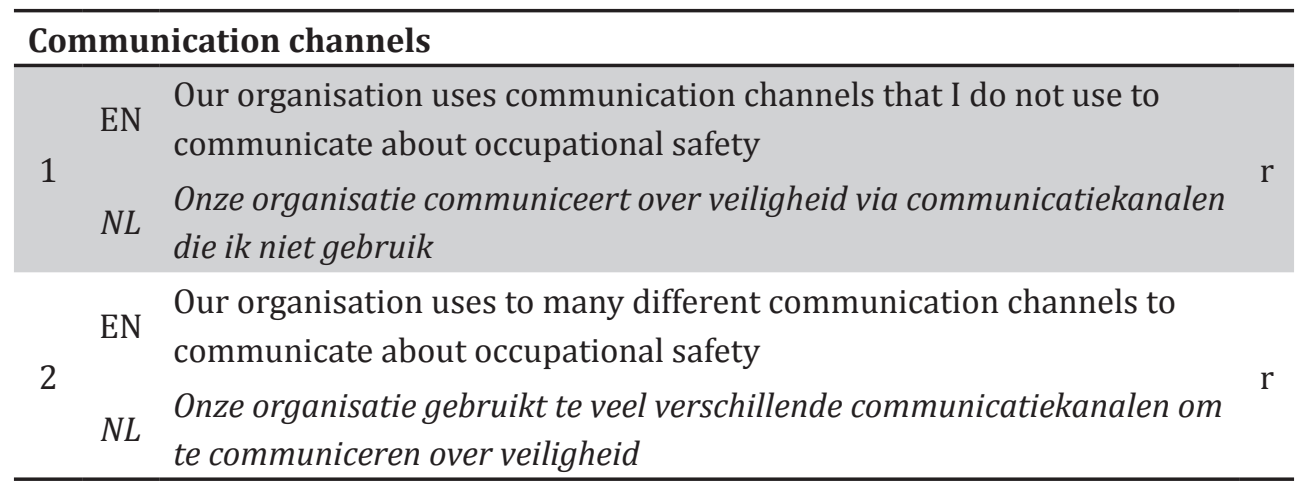

Note: $\mathrm{r}=$ recoded item

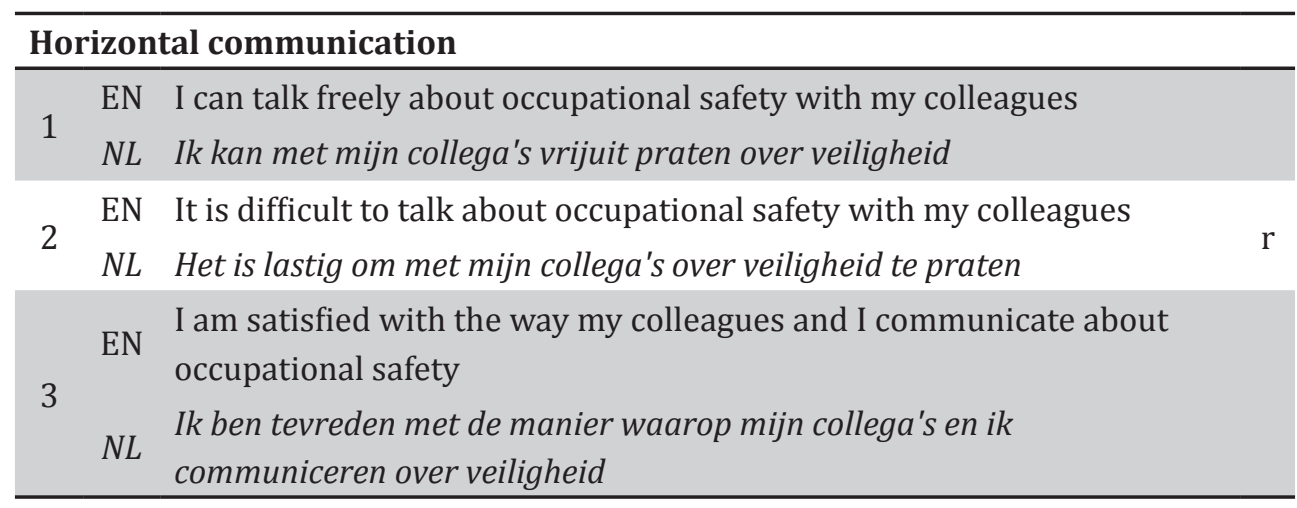

Note: $\mathrm{r}=$ recoded item

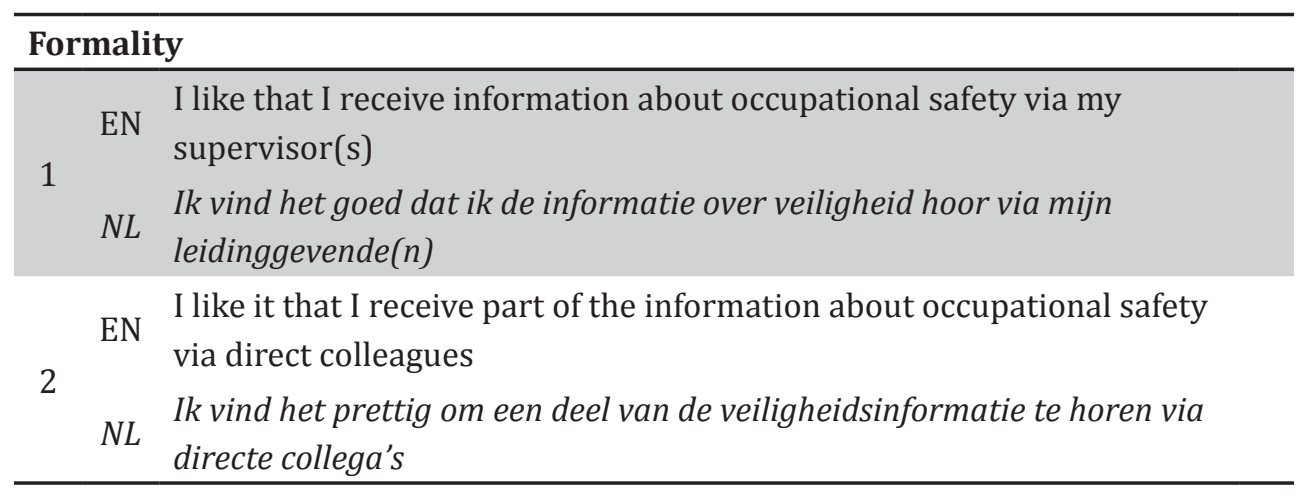

Note: The internal reliability (Cronbach's alpha coefficient) for Formality was <.7. 



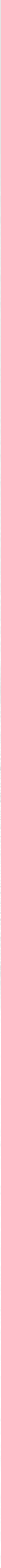




\section{Summary}




\section{Summary}

With over 374 million occupational accidents and 2.78 million fatalities annually worldwide (International Labour Organization, 2018), workplace safety is a problematic and persistent issue. Considering the impact of these accidents on employees, their colleagues, friends, family and organisations, this dissertation aims to contribute to safer workplaces by increasing current insights and understandings of occupational safety. It does so by applying various research methods to develop a better understanding of personal, social and organisational aspects that (potentially) influence occupational safety.

In the past hundred years a wide variety of approaches and factors have been used in an attempt to improve safety in the workplace. During the first half of the $20^{\text {th }}$ century two hypotheses regarding accidents were dominant: The environmental hypothesis, in which accidents are attributed to external factors (e.g., labour conditions), and the individual hypothesis, which is based on the belief that some people are more prone to accidents than others (Swuste et al., 2010). After the Second World War new scientific insights led to different approaches to occupational safety between the scientific and professional domain. Whereas the scientific focus shifted from a psychological view towards a combination of technology, ergonomics and epidemiology (Swuste et al., 2014), the professional fields sticks with a human failure approach. While during the 1970s some steps were made to integrate the insights and knowledge from different disciplines, these two distinct approaches largely remain to date. These different approaches and their wide variety of studied factors call for a thorough overview of what has been studied to date. Therefore, this dissertation starts with the question:

\section{RQ 1: What is the current status of occupational safety research with regard to determinants of safety outcomes and performance?}

Considering the importance of communication for the effective organisation and coordination of work, it is no wonder that communication is also important for the safe execution of work (e.g., Quinn \& Dutton, 2005; Vassie \& Lucas, 2001). Whereas safety communication is mainly associated with 
supervisor-subordinate communication, advancing insights have shown that a more comprehensive understanding of safety communication is needed for it to become effective. Furthermore, there is still debate on how safety communication relates to other safety variables such as safety climate, safety culture and safety performance. Whereas some state the relation with safety performance to be direct, others assume that safety communication affects safety performance through safety climate or safety culture. Lastly, given the societal and organisational changes that have taken place over the past decades (e.g., globalisation, increasingly loose labour market) communication is becoming increasingly important for safety in the workplace. In order to contribute to current understandings of safety communication the following overall research question is formulated:

\section{RQ 2: What is the role of communication in occupational safety?}

This dissertation starts by exploring the different factors that have been studied in occupational safety research through a systematic literature review in order to answer the first research question (Chapter 2). Based on the outcomes areas for further research were identified. Chapters 3 and 4 describe qualitative studies that focus on supervisors and managers and their perceptions of occupational safety, whereas chapters 5 and 6 focus on safety communication in relation to employees.

Chapter 2 presents a systematic review of research conducted in four highrisk industries between 1980 and 2016. Through a systematic and step-wise procedure the 27.527 records extracted from four online bibliographical databases were reduced to 176 peer-reviewed articles. Subsequently, the variables studied in these articles and the nature of their relationships (i.e., positive, negative, or nonsignificant) were extracted, grouped and classified, resulting in 1.946 relationships between 84 variables. These variables were then clustered into seven clusters, comprising a total of 20 categories. The results indicate that the far majority of studied relationships between variables either are subject to debate or appear to be understudied. However, consensus is found for the negative relationship between safety performance and negative safety outcomes (i.e., incidents, accidents and injuries), safety compliance and negative safety outcomes, and psychophysical states and negative safety outcomes, indicating that more or better performance, 
compliance, and psychophysical states are related to fewer incidents, accidents, and injuries. Working hours and lifestyle disorders on the other hand, were positively related to negative safety outcomes, indicating that an increase in these variables is associated with an increase in incidents, accidents, or injuries. Consensus was also found for the positive relationships between seven variables and safety performance, indicating that more or higher (safety) control, safety climate, management behaviours, (safety) communication, safety policies and procedures, psychophysical states, and safety knowledge (sharing) are associated with better performance. One of the most interesting clusters is that of the influence of management and colleagues and, more specifically, the results regarding the management of safety. In light of organisational efforts to improve occupational safety, the results regarding management attitudes \& behaviours and management of safety were further explored through semi-structured interviews with supervisors.

Chapter 3 builds on the outcomes of the systematic literature review from Chapter 2 by exploring supervisors' beliefs and attitudes towards (the management of) occupational safety through semi-structured, in-depth interviews $(N=22)$. The results illustrate that safety is perceived to play a substantial and (increasingly) important role in supervisors' job, although they have to balance safety with other organisational objectives. Whereas the majority of supervisors believes the motivation for safety within their organisation to be sincere, some supervisors point out that this motivation is predominantly external. Half of the supervisors differentiate between an overall responsibility for safety and a final responsibility, with the latter being attributed to those higher in hierarchy. Supervisors' beliefs regarding the preventability of accident, accident reasons, and incident reporting were rather negative and fatalistic. Whereas none of the supervisors believed that incidents and accidents could be completely prevented, they agreed with one another that incident prevention should be the aim of the organisation. Accidents were mostly thought to be the result of unconscious actions or behaviours for which the consequences were not foreseen, although other factors such as time, a lack of focus and old habits were also mentioned. Supervisors did not think that all incidents were reported, and were especially sceptical about the reporting of minor incidents. They did think that incident reporting could be improved by increasing the visibility of, and follow-ups on incident reports and by providing more insights into the purpose and 
importance of reporting. Unexpectedly, supervisors perceived a problematic gap between field employees and employees working in the office related to a mutual dependency. Lastly, supervisors' attitudes towards the safety management instruments from Chapter 2 were assessed. In general, the findings here were in line with the outcomes described in Chapter 2 as the supervisors expressed positive attitudes towards the use of inspections, accident registration and accident analysis, training and safety-related activities. However, the supervisors were divided on the use of sanctions and rewards, and they were critical with regard to the use of (unwritten) rules and regulations. Despite their predominantly positive attitudes, the supervisors had concerns with regard to the application of these instruments, and they believed their effectiveness could be improved. Noteworthy is that communication emerged as a crucial factor for the management of occupational safety. Not only was communication viewed by supervisors as an effective mean on its own, they also stressed its importance for other safety management instruments.

Chapter 4 further explores the role of safety in supervisors' work to increase understandings of what drives safety-related thinking, decisions and behaviours. By applying the ideal institutional orders framework as proposed by Thornton et al. (2013) to qualitative data gathered through semi-structured, in-depth interviews with managers the aim was to identify, illustrate and explain the potentially different rationales for safety-related behaviours and decisions. In essence, institutional logics provide 'guidelines on how to interpret and function in social situations' (Greenwood et al., 2011, p. 318). Multiple of such logics can be simultaneously present and depending on the degree of logic compatibility (i.e., the extent to which logics provide (in)compatible prescriptions for action), and the degree of logic centrality (i.e., the extent to which one or multiple logics are core to organisational functioning), logic multiplicity may lead to institutional complexity. The results demonstrate that an institutional logics perspective can be effectively applied to identify the different rationales (i.e., logics) towards occupational safety. The market logic was reflected in the use of safety performance as a means to increase consumer preference and in the effects of market competitiveness on occupational safety. The professional logic was reflected in prioritising staying safe over getting the work done and vice versa, based on assessments of individual situations. Lastly, the corporation logic was reflected in managers' use of hierarchical power to determine the content and 
planning of work. These examples not only illustrate the different rationales that underlie safety-related behaviours and decisions, but also how they differ between managers. Next to examples of individual logics in managers' quotes, examples of logic multiplicity between the logics of the market, profession, and corporation emerged. Institutional complexity between the market and profession logics became apparent through the incompatibility of the selfinterested focus on increasing (efficiency) profits of the market logic and the professional logic's urge to adhere to high quality work standards. The complexity between the market logic and corporation logic was the result of the incompatibility between the market logic's self-interested focus on increasing (efficiency) profits and the corporation's logic in which managers have to uphold their hierarchical status and responsibilities. Lastly, complexity between the professional logic and the logic of the corporation arose due to the incompatibility between corporate work routines and the actual work that needed to be done.

The studies presented in Chapters 3 and 4 provide an in-depth exploration of variables related to managers perspectives regarding (the management of) safety. However, in the organisational setting managers are not the ones most at risk. Considering the (perceived) importance of communication (Chapters 2 and 3) and the importance of employees' safety performance for occupational safety (Chapter 2), ways through which employees can be best approached and stimulated to work safely are further explored through quantitative research.

Chapter 5 explores the potential of distinguishing between different levels of ability and motivation for occupational safety. By means of a quantitative questionnaire among employees of a logistics warehouse $(N=77)$, the influence of three levels of motivation and ability (i.e. personal, social, and structural) on safety climate and safety performance was assessed, aiming to increase insights in how these elements can be effectively used to improve occupational safety. Although the analyses only allowed for differentiation between personal and external levels of motivation and ability, the results illustrate that the inclusion of different levels increased the predictive power of motivation and ability for safety climate and safety performance, compared to using the overall constructs of motivation and ability. Specifically, the external level of ability proved important for levels of safety climate, while both the personal level of motivation and the external level of ability 
proved important for levels of safety performance. These findings imply that motivation and ability should not be approached as the singular constructs they are currently thought to be. In improving perceptions of safety and safety performance of their employees, organisations should focus on personal motivation (e.g., in safety instructions) and external ability (e.g., providing proper safety equipment and safety training).

Chapter 6 describes the development and initial validation of a safety communication measurement instrument. The narrow and unilateral view on safety communication in existing measurement scales is at odds with the (perceived) importance of communication for occupational safety as highlighted in the different chapters of this dissertation. Building on safety communication literature, existing safety communication measures, and the input from expert rounds, 22 safety communication-related topics were identified. These topics formed the basis for an initial item pool of 134 items that was provided to a development sample $(N=252)$ in the form of a questionnaire. Principal component analysis (PCA) revealed an underlying structure of safety communication consisting of 13 distinctive factors: message, vertical communication, sender credibility, reach, exemplary behaviours, timing, match with practice, tone, feedback, dissemination failures, communication channels, horizontal communication, and formality. Subsequent bivariate and partial correlation analyses to explore the relationships of these factors with safety climate, safety motivation, and safety knowledge revealed a (partial) overlap between the safety communication variables and safety climate. However, even when the variance shared with safety climate is controlled for, sender credibility emerges as an important factor for safety motivation, while vertical communication, reach, tone, communication channels, and horizontal communication emerge as important factors for safety knowledge. These findings illustrate the importance of a more comprehensive understanding of safety communication and the resulting measurement instrument provides researchers and practitioners with a comprehensive instrument to assess employee perceptions of organisational safety communication.

The combined outcomes of the different studies presented in this dissertation have several overarching theoretical and practical implications. First, occupational safety is subject to the dynamics of the organisation and the influences of the field in which it operates. Aside from the wide variety of 
different factors that have the potential to influence occupational safety (Chapter 2), occupational safety has to compete with other organisational objectives within the organisation (Chapter 3 ). In addition, safety-related thinking, decisions and behaviour are constantly influenced by actors from within and outside of the organisation. Employees continuously have to balance the different interests regarding occupational safety as provided by the logics of the market, profession and corporation (Chapter 4). The (perceived) priority of occupational safety might be further influenced by ambiguous beliefs regarding the motivation within the organisation to pursue occupational safety, the responsibility for occupational safety, and the preventability of occupational incidents (Chapter 3). Practitioners are recommended to adopt an integrative approach towards occupational safety that includes both technical and human factors. They are recommended to prioritise occupational safety and align the different perceptions, beliefs and prescriptions from institutional logics toward this priority.

Second, occupational safety is a very complex and broad issue. Over the years practitioners and researchers have adopted different approaches and applied a wide variety of different factors in their attempts to improve workplace safety (Chapters 1 and 2). The extensive amount of ambiguity regarding the relationships of studied factors with safety outcomes and safety performance (Chapter 2) further complicates the search for an effective solution to workplace incidents. Even when the effectivity of a specific factor has been demonstrated in several studies an effective implementation may be hindered by personal perceptions or beliefs (Chapter 3), conflicting prescriptions from institutional logics (Chapter 4), and the different levels at which factors can operate (Chapter 5). Before implementing safety policies, interventions or safety management instruments, managers and practitioners are recommended to conduct a thorough analysis of the expected effectivity and the perceptions and beliefs of their employees regarding said policies, interventions and instruments.

Third, safety communication emerges as an important factor for the improvement of occupational safety. Not only did the majority of studies underline the positive influence of communication on safety performance (Chapter 2), its importance for both occupational safety and other safety management instruments was also underlined by supervisors (Chapter 3 ). Despite its perceived importance, it was revealed that a clear and common 
agreed upon conceptualisation of safety communication and its relationships with other safety-related variables is lacking. The outcomes of the development and initial validation of a safety communication measurement instrument (Chapter 6) seem to confirm the hypothesised overlap between safety communication and safety climate. However, the results also indicate that several aspects of safety communication (i.e., sender credibility, vertical communication, reach, tone, communication channels, and horizontal communication) explain additional variance in safety motivation and safety knowledge compared to safety climate. These findings highlight the need for a more comprehensive understanding of safety communication and additional research to study its potential. Especially considering the potential of communication in addressing specific levels of motivation and knowledge (Chapter 5).

In sum, the outcomes of the different studies provided in this dissertation illustrate the complexity of occupational safety, and the potential of communication for improving occupational safety. 


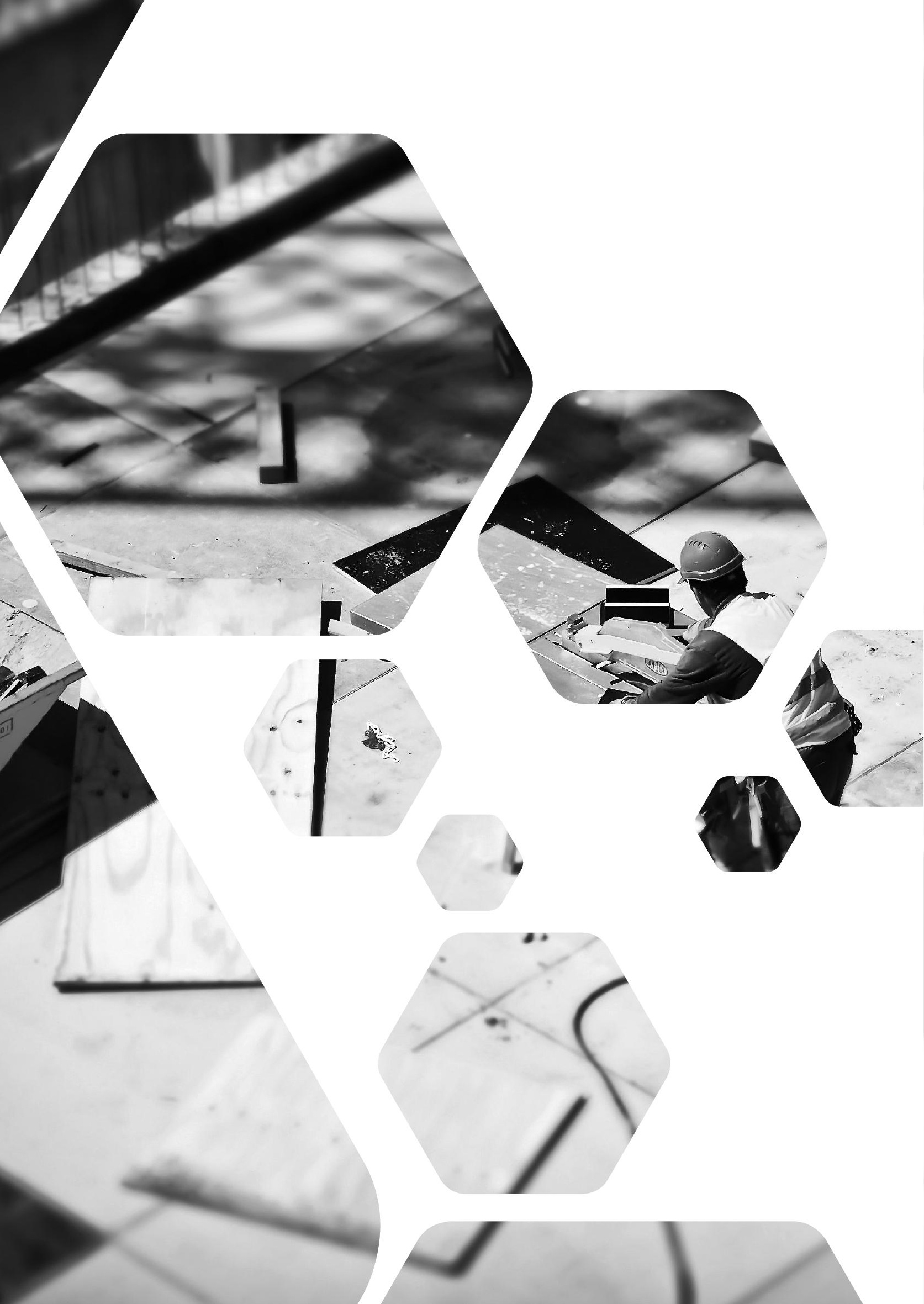


Samenvatting

(summary in Dutch) 


\section{Samenvatting (summary in Dutch)}

Met naar schatting meer dan 374 miljoen arbeidsongevallen en 2,78 miljoen dodelijke slachtoffers wereldwijd (International Labour Organization, 2018) is arbeidsveiligheid een probleem met een enorme impact op medewerkers, hun familie en vrienden en organisaties. Dit proefschrift heeft tot doel om door het vergroten van de inzichten in arbeidsveiligheid, bij te dragen aan het creëren van veilige werkomgevingen. Het onderzoek in dit proefschrift richt zich hierbij op persoonlijke, sociale en organisatorische aspecten die van invloed kunnen zijn op arbeidsveiligheid.

In de afgelopen honderd jaar hebben onderzoekers en professionals arbeidsveiligheid vanuit verschillende perspectieven benaderd en hebben zij een breed scala aan factoren onderzocht in een poging om werkomgevingen veiliger te maken. In de eerste helft van de $20^{\mathrm{e}}$ eeuw zijn hierin twee verschillende benaderingen dominant: de omgevingshypothese, waarin ongevallen worden toegeschreven aan externe factoren zoals arbeidsomstandigheden, en de individuele hypothese, die gestoeld is op de overtuiging dat sommige mensen vatbaarder zijn voor ongevallen dan anderen (Swuste et al., 2010). Na de Tweede Wereldoorlog ontstond er een breuk tussen de wetenschap en het professionele domein. Waar de wetenschap arbeidsveiligheid benadert vanuit een combinatie van nieuwe inzichten uit de technologie, ergonomie en epidemiologie, houdt het professionele domein vast aan de individuele hypothese, die ongevallen toeschrijft aan menselijk falen (Swuste et al., 2014). En hoewel er in de jaren '70 van de vorige eeuw stappen zijn gezet om de inzichten en kennis vanuit verschillende disciplines te integreren, blijven de twee benaderingen grotendeels naast elkaar bestaan. De verschillende perspectieven van waaruit arbeidsveiligheid wordt benaderd, gecombineerd met de grote verscheidenheid aan onderzochte factoren, vragen om een grondig overzicht van wat tot nu toe bekend is over arbeidsveiligheid. Zeker gezien de steeds complexer wordende werkomgeving. De eerste vraag in dit proefschrift luidt daarom als volgt:

\section{OZV 1: Wat is de huidige stand van zaken in het onderzoek naar arbeidsveiligheid met betrekking tot determinanten van veiligheidsuitkomsten (incidenten, ongelukken en verwondingen) en veiligheidsprestaties (safety performance)?}


Gezien het belang van communicatie voor de organisatie en coördinatie van werk, is het niet verwonderlijk dat communicatie ook belangrijk wordt bevonden voor een veilige uitvoering van dat werk (Quinn \& Dutton, 2005; Vassie \& Lucas, 2001). Momenteel bestaat er echter geen eenduidige definitie van veiligheidscommunicatie. Hoewel voortschrijdende inzichten hebben aangetoond dat een bredere kijk op veiligheidscommunicatie noodzakelijk is voor het verbeteren van arbeidsveiligheid, blijft een beperkte opvatting van veiligheidscommunicatie als de interactie tussen leidinggevende en medewerkers overheersen. Daarnaast bestaat er onduidelijkheid over hoe veiligheidscommunicatie zich verhoudt tot andere variabelen zoals veiligheidsklimaat (safety climate), veiligheidscultuur (safety culture) en veiligheidsprestaties (safety performance). Waar sommige wetenschappers stellen dat de relatie tussen veiligheidscommunicatie en veiligheidsprestaties direct is, stellen anderen dat deze relatie gemedieerd wordt door het heersende veiligheidsklimaat of de veiligheidscultuur binnen de organisatie. Maatschappelijke veranderingen vergroten ondertussen het belang van communicatie voor een veilige werkomgeving doordat een mengeling van verschillende culturen en talen steeds vaker voorkomt (globalisering) en arbeidsrelaties tussen werkgevers en werknemers steeds minder vast zijn. Om de huidige kennis van en inzichten in veiligheidscommunicatie te vergroten staat de volgende onderzoeksvraag centraal in dit proefschrift:

\section{OZV 2: Welke rol speelt communicatie bij arbeidsveiligheid?}

Dit proefschrift begint met een onderzoek dat op systematische wijze de verschillende factoren in kaart brengt die zijn onderzocht in relatie tot arbeidsveiligheid en veiligheidsprestaties (hoofdstuk 2). De uitkomsten hiervan worden enerzijds gebruikt om de eerste onderzoeksvraag (OZV 1) te beantwoorden en daarnaast om aanknopingspunten voor vervolgonderzoek te identificeren. $O p$ basis van de uitkomsten van hoofdstuk 2 richten hoofdstukken 3 en 4 zich op de percepties en attitudes van leidinggevenden en managers. In hoofdstukken 5 en 6 ligt de focus op de bijdrage van veiligheidscommunicatie om medewerkers te bereiken.

Hoofdstuk 2 beschrijft een systematische review van 176 studies naar arbeidsveiligheid die tussen 1980 en 2016 zijn uitgevoerd in de bouw, (logistieke) magazijnen, de maakindustrie en de (offshore) petrochemie. De 
variabelen en hun onderlinge relaties (positief, negatief of niet significant) die onderzocht zijn in deze studies werden in kaart gebracht en vervolgens gegroepeerd. Uit het overzicht blijkt dat verreweg het grootste deel van het onderzoek naar arbeidsveiligheid zich heeft gericht op het bestuderen van de invloed van de verschillende factoren op veiligheidsprestaties (safety performance) en incidenten, ongelukken en letsel (safety outcomes). Hierbinnen blijkt de nadruk te liggen op factoren die zijn gerelateerd aan management en collega's, kenmerken van werk(plekken) en arbeidsomstandigheden en demografische kenmerken van medewerkers. Voor verreweg het grootste gedeelte van de relaties tussen deze factoren blijken de uitkomsten niet eenduidig te zijn. Uitgaande van een twee-derde meerderheid werd er voor minder dan zeven procent van de relaties met negatieve veiligheidsuitkomsten en voor minder dan elf procent van de relaties met veiligheidsprestaties een eenduidige uitkomst gevonden. Aangezien het cluster management en collega's veel factoren bevat die (in zekere mate) beïnvloed kunnen worden door actoren binnen de organisatie, vormt het een uitermate interessant cluster voor vervolgonderzoek.

Hoofdstuk 3 bouwt voort op de resultaten van hoofdstuk 2 en beschrijft een kwalitatief onderzoek naar verschillende factoren uit het management en collega's cluster. Door middel van semigestructureerde interviews worden de percepties, attitudes en overtuigingen van leidinggevenden $(N$ $=22$ ) ten aanzien van (het managen van) arbeidsveiligheid onderzocht. De resultaten laten ten eerste zien dat arbeidsveiligheid een complexe rol speelt in het werk van leidinggevenden. Ondanks de belangrijke rol die arbeidsveiligheid in hun werk speelt, moeten leidinggevenden veiligheid zien te combineren met andere doelstellingen van de organisatie. Ten tweede bestaan er zowel tussen de verschillende hiërarchische niveaus als tussen individuele leidinggevenden verschillen in de overtuigingen ten aanzien van veiligheid. Waar het merendeel van de leidinggevenden ervan overtuigd is dat de motivatie voor arbeidsveiligheid binnen de organisatie oprecht en intrinsiek is, zijn er ook leidinggevenden die hieraan twijfelen. Wel zijn vrijwel alle leidinggevenden het erover eens dat iedereen een eigen verantwoordelijkheid voor arbeidsveiligheid heeft, al maakt de helft van hen wel onderscheid tussen een algemene verantwoordelijkheid voor veiligheid en de eindverantwoordelijkheid. Hierbij wordt de eindverantwoordelijkheid vaak hoger in de organisatie neergelegd. Geen van de leidinggevenden denkt dat het mogelijk is om alle arbeidsongevallen te voorkomen en veelal zijn 
ze van mening dat ongevallen het gevolg zijn van onbewuste acties. Een meerderheid is daarnaast van mening dat niet alle voorvallen gemeld worden; vooral kleinere voorvallen zullen minder worden gemeld. Tot slot maken de resultaten inzichtelijk hoe leidinggevenden aankijken tegen het managen van arbeidsveiligheid en hoe hun attitudes hierop van invloed zijn. Zo wordt de individuele bereidheid om bepaalde middelen in te zetten beïnvloed door de gepercipieerde effectiviteit van het middel. Van de onderzochte middelen komt communicatie naar voren als één van de belangrijkste en meest effectieve manieren om arbeidsveiligheid te verbeteren. Niet alleen zijn leidinggevenden van mening dat communicatie op zichzelf een belangrijk middel is om de veiligheid te verbeteren, ze zijn ook van mening dat het onmisbaar is voor de effectiviteit van andere middelen zoals inspecties. Gezien de verschillende overtuigingen die leidinggevenden hadden ten aanzien van de motivatie voor arbeidsveiligheid binnen de organisatie en het feit dat ze van mening waren dat de meeste ongevallen het resultaat zijn van onbewuste acties is meer onderzoek nodig. Om inzicht te krijgen in hoe verschillende motivaties en rationales van invloed zijn op beslissingen van leidinggevenden met betrekking tot veiligheid en op hun veiligheids-gerelateerd denken en gedrag werd een tweede kwalitatief onderzoek uitgevoerd.

Hoofdstuk 4 beschrijft de resultaten van een studie waarin een institutional logics perspectief is toegepast op de kwalitatieve data verzameld onder managers $(N=22)$. Het onderzoek had tot doel om de verschillende beweegredenen voor arbeidsveiligheid bloot te leggen en uit te leggen hoe de verschillende beweegredenen ten grondslag liggen aan veiligheids-gerelateerd denken, beslissingen en gedrag. De resultaten laten zien dat een institutional logics perspectief succesvol kan worden toegepast op arbeidsveiligheid. Ten eerste legde het institutional logics perspectief de verschillende logica's van de markt, de professie en de corporatie bloot in de denkwijze, beslissingen en gedragingen van managers. Ten tweede werden door het toepassen van een institutional logics perspectief ook voorvallen gevonden waarin de logica's van de markt, de professie en de corporate gelijktijdig voorkwamen (logic multiplicity). Het aantonen van de aanwezigheid van verschillende logica's op hetzelfde moment maakt inzichtelijk hoe veiligheids-gerelateerde conflicten binnen een organisatie, afdeling of team kunnen ontstaan. Ook zijn de verschillende manieren waarop managers deze conflicten oplossen in kaart gebracht. De aangetoonde toepasbaarheid van een institutional logics perspectief op arbeidsveiligheid biedt onderzoekers en professionals een 
nieuw raamwerk dat hen in staat stelt om schijnbaar irrationeel of onbewust veiligheids-gerelateerd denken, beslissingen en gedrag te benoemen en beter te begrijpen.

Hoewel de studies zoals beschreven in hoofdstuk 3 en hoofdstuk 4 beide bijdragen aan het verbeteren van huidige inzichten in het (ervaren) belang van arbeidsveiligheid voor managers en leidinggevenden, vormen managers en leidinggevenden niet de groep die het meeste risico loopt op de werkvloer. Om managers en leidinggevenden beter in staat te stellen om de arbeidsveiligheid te verbeteren en de veiligheid van werknemers te vergroten is meer inzicht nodig in hoe werknemers het beste kunnen worden bereikt, gemotiveerd en in staat gesteld om veilig te werken.

Hoofdstuk 5 beschrijft een kwantitatieve studie naar de verschillende niveaus van motivatie en vaardigheid en de relatie met veiligheidsklimaat en veiligheidsprestaties. Hoewel het belang van motivatie en vaardigheid al lange tijd erkend wordt, worden ze tot op heden altijd geconceptualiseerd als eendimensionale concepten. Met behulp van een vragenlijst werd een onderzoek uitgevoerd onder medewerkers $(N=77)$ van een logistiek magazijn waarbij hun motivatie en vaardigheid ten aanzien van arbeidsveiligheid op drie verschillende niveaus (persoonlijk, sociaal en extern) werd gemeten. Daarnaast werd ook gemeten hoe zij scoorden op veiligheidsklimaat en veiligheidsprestaties. De resultaten bevestigden de relatie tussen veiligheidsklimaat en veiligheidsprestaties. Hoewel niet alle niveaus van motivatie en vaardigheid konden worden aangetoond, bleek dat door het meten van motivatie en vaardigheid op twee niveaus (persoonlijk en extern) de voorspellende waarde van deze variabelen voor zowel veiligheidsklimaat als veiligheidsprestaties toenam. Voor veiligheidsklimaat bleek externe vaardigheid (bijvoorbeeld door het verstrekken van de juiste veiligheidsuitrusting en trainingen) het meest relevant, waar voor veiligheidsprestaties zowel het persoonlijke niveau van motivatie (bijvoorbeeld door in veiligheidsinstructie motiverende elementen toe te voegen) als de externe vaardigheid relevant waren.

Hoofdstuk 6 beschrijft de ontwikkeling van een meetinstrument (vragenlijst) voor veiligheidscommunicatie. Hoewel de resultaten van de voorgaande studies het (gepercipieerde) belang en de effectiviteit van communicatie voor arbeidsveiligheid aantonen, is de benadering van 
veiligheidscommunicatie in bestaande meetinstrumenten vrij beperkt. Om een beter en breder beeld te krijgen van veiligheidscommunicatie, had deze studie tot doel om een nieuw meetinstrument voor veiligheidscommunicatie te ontwikkelen. Op basis van wetenschappelijke literatuur, bestaande veiligheidscommunicatieschalen en kwalitatieve input van experts werden 22 thema's geïdentificeerd die gerelateerd zijn aan veiligheidscommunicatie. Deze thema's dienden als startpunt voor het maken van items. Nadat deze items waren voorgelegd aan experts op het gebied van communicatie en professionals op het gebied van veiligheidscommunicatie werd de vragenlijst vooraf getest met 15 medewerkers van een industriële dienstverlener. Hierna werd de vragenlijst afgenomen onder medewerkers $(N=252)$ van vier organisaties. Op basis van een Principal Component Analysis kwamen 13 veiligheidscommunicatiefactoren naar voren, waarvan 12 factoren een goede interne betrouwbaarheid (Cronbach's alpha) hadden. Deze factoren vormden een afspiegeling van werknemersbeoordelingen van de gecommuniceerde boodschap, verticale communicatie, geloofwaardigheid van de zender, bereik, voorbeeldgedrag van managers, timing van de communicatie, afstemming van de communicatie met de praktijk, gebruikte toon in de communicatie, mogelijkheden tot het geven van feedback, fouten in de verspreiding van informatie, gebruikte communicatiekanalen binnen de organisatie en horizontale communicatie. De daaropvolgende (bivariate en partial) correlatieanalyses maken inzichtelijk dat de veiligheidscommunicatievariabelen, in lijn met eerdere bevindingen, veel overlap vertoonden met veiligheidsklimaat. Zelfs wanneer er gecontroleerd werd voor de met veiligheidsklimaat gedeelde variantie, correleerde de geloofwaardigheid van de zender echter aanzienlijk met de veiligheidsmotivatie. Bovendien bleek dat, zelfs wanneer er gecontroleerd werd voor de met veiligheidsklimaat gedeelde variantie, verticale communicatie, bereik, toon, communicatiekanalen en horizontale communicatie nog steeds significant correleerden met veiligheidskennis. Het in deze studie ontwikkelde meetinstrument voor veiligheidscommunicatie draagt bij aan een beter en breder begrip van veiligheidscommunicatie en biedt onderzoekers en professionals een praktisch instrument om in kaart te brengen hoe de veiligheidscommunicatie binnen organisaties wordt beoordeeld door medewerkers.

Op basis van de in dit proefschrift gepresenteerde resultaten kunnen enkele overkoepelende conclusies worden getrokken. Ten eerste blijkt 
dat arbeidsveiligheid sterk beïnvloed wordt door de dynamiek binnen de organisatie en door invloeden van het veld waarin de organisatie actief is. Los van de grote diversiteit aan factoren die mogelijk invloed hebben op arbeidsveiligheid (hoofdstuk 2) laten de resultaten zien dat arbeidsveiligheid binnen de organisatie moet concurreren met andere doelstellingen (hoofdstuk 3). Daarnaast wordt het denken over veiligheid en veiligheidsgerelateerde beslissingen en gedragingen voortdurend beïnvloed door actoren binnen en buiten de organisatie. Medewerkers maken continu (al dan niet bewust) afwegingen tussen de verschillende belangen die worden opgelegd vanuit de markt, de professie en de organisatie (hoofdstuk 4). De (gepercipieerde) prioriteit om te werken aan het verbeteren van de arbeidsveiligheid kan verder worden beïnvloed door ambigue opvattingen die medewerkers hebben ten aanzien van de motivatie voor arbeidsveiligheid binnen de organisatie, de verantwoordelijkheid voor arbeidsveiligheid binnen de organisatie en de vraag of arbeidsongelukken te voorkomen zijn (hoofdstuk 3). Om hiermee om te gaan raden we professionals aan om een integrale aanpak ten aanzien van veiligheid te hanteren waarin zowel technische aspecten als aspecten gerelateerd aan houding, kennis en gedrag worden meegenomen. Arbeidsveiligheid zou eenduidig de prioriteit moeten krijgen binnen organisaties en eventuele afwijkende rationales van de markt, de professie of de organisatie (institutional logics) zouden onderling moeten worden afgestemd zodat ze geen afbreuk doen aan de veiligheid van de werkomgeving.

Ten tweede blijkt arbeidsveiligheid een zeer complexe en brede thematiek. In de loop der tijd hebben professionals en onderzoekers arbeidsveiligheid vanuit diverse invalshoeken benaderd en hebben ze via een breed scala aan factoren gepoogd om de veiligheid op de werkplek te verbeteren (hoofdstukken 1 en 2). De ambiguïteit ten aanzien van de effectiviteit van de meerderheid van de onderzochte variabelen (hoofdstuk 2) maakt het voor zowel onderzoekers als professionals moeilijk om tot een effectieve aanpak te komen om de veiligheid in de werkomgeving te verbeteren. Zelfs wanneer de effectiviteit van een specifieke factor in verschillende studies is aangetoond, kan een effectieve implementatie worden belemmerd door persoonlijke percepties of overtuigingen (hoofdstuk 3), conflicterende voorschriften (hoofdstuk 4) en de verschillende niveaus waarop factoren worden ingezet (hoofdstuk 5). Leidinggevenden en (veiligheids)professionals wordt aangeraden om, voordat ze veiligheidsmaatregelen, interventies of middelen voor veiligheidsbeheer implementeren, een grondige analyse uit te voeren van 
de verwachte effectiviteit en de attitudes van werknemers ten aanzien van de te implementeren maatregelen.

Ten derde blijkt veiligheidscommunicatie een belangrijke factor te zijn voor de verbetering van arbeidsveiligheid. Niet alleen werd de positieve invloed van communicatie op arbeidsveiligheid in een meerderheid van de in hoofdstuk 2 onderzochte studies onderschreven, ook leidinggevenden onderschreven het belang van communicatie voor zowel arbeidsveiligheid als voor andere veiligheidsbeheersinstrumenten (hoofdstuk 3). Ondanks het (gepercipieerde) belang van veiligheidscommunicatie, werd duidelijk dat een eenduidige en algemeen geaccepteerde conceptualisering van veiligheidscommunicatie ontbreekt. Ook bestaat er nog veel onduidelijkheid over de manier waarop veiligheidscommunicatie zich verhoudt tot andere veiligheidsgerelateerde variabelen. De resultaten van de ontwikkeling en eerste validatie van een meetinstrument voor veiligheidscommunicatie (hoofdstuk 6) lijken de veronderstelde overlap tussen veiligheidscommunicatie en veiligheidsklimaat te bevestigen. De resultaten geven echter ook aan dat verschillende aspecten van veiligheidscommunicatie (de geloofwaardigheid van de zender, verticale communicatie, bereik, toon, communicatiekanalen en horizontale communicatie) van belang zijn voor veiligheidsmotivatie en veiligheidskennis, zelfs wanneer er gecontroleerd wordt voor de invloed van veiligheidsklimaat. Deze resultaten laten zien dat aanvullend onderzoek nodig is om het potentieel van veiligheidscommunicatie voor het verbeteren van arbeidsveiligheid nader te onderzoeken. Des te meer gezien het potentieel van communicatie bij het adresseren van specifieke niveaus van motivatie en vaardigheid (hoofdstuk 5).

Samengevat laten de resultaten van de verschillende studies in dit proefschrift de complexiteit van arbeidsveiligheid en het potentieel van communicatie voor het verbeteren hiervan zien. 


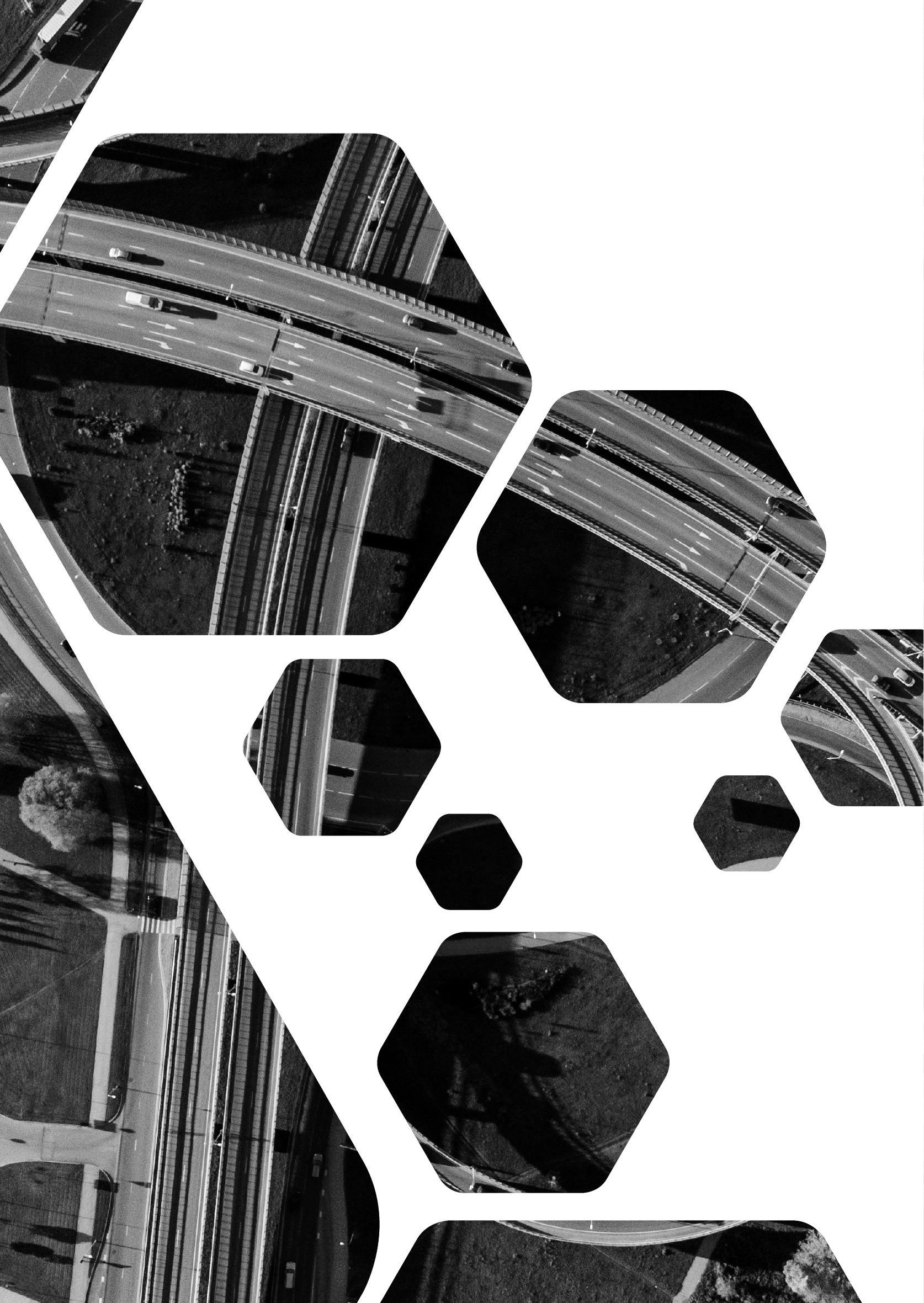




\section{Bio-bliography}




\section{Bio-bliography}

Pieter Cornelissen was born on November 23, 1987 in Thorn, the Netherlands. After completing his secondary schooling at the Rythovius College high school in Eersel, he moved to Enschede in 2006 to study Communication Science at the University of Twente in Enschede. After obtaining his Bachelor degree, Pieter followed courses from both the marketing communication specialisation and the corporate communication specialisation tracks at the University of Twente. Pieter finished his master in Communication Studies (cum laude) with a thesis on the effectiveness of a behavioural change model aimed at occupational safety in a large logistics warehouse.

While this behavioural change model did not prove to be effective, it did spark Pieter's interest, and that of his supervisors, in occupational safety. In cooperation with Joris van Hoof a PhD research proposal was written, through which Pieter obtained a PhD position at the Communication Science research group chaired by Professor Menno de Jong. Between August 2014 and August 2018 Pieter was employed as a PhD student and teacher at the faculty of Behavioural, Management and Social Sciences (BMS) at the University of Twente. In addition to the studies undertaken in the context of this dissertation, Pieter was involved as a teacher in the course Research Projects, and TEM modules 2, 4, and 7. He also supervised several bachelor students and master students during their research. Currently, Pieter is employed at Strukton Rail as a Business Improvement and Safety, Health and Environment consultant. 


\section{Scientific publications}

Cornelissen, P. A., Van Hoof, J. J., \& Van Vuuren, M. (2014). Enabling employees to work safely: The influence of motivation and ability in the design of safety instructions. Technical Communication, 61(4), 232-244.

Cornelissen, P. A., Van Hoof, J. J., \& De Jong, M. D. T. (2017). Determinants of safety outcomes and performance: A systematic literature review of research in four high-risk industries. Journal of Safety Research, 62, 127141. doi: 10.1016/j.jsr.2017.06.009

\section{Under review}

Cornelissen, P.A., Van Vuuren, M., \& Van Hoof, J.J. How logical is safety? An institutional logics perspective on occupational safety.

\section{Conferences}

Cornelissen, P. A. (2015). Veilig gedrag op de Werkplek: Wat weet de wetenschap? Paper presented at the biennial National Safety At Work Event, Rotterdam, the Netherlands.

Cornelissen, P. A. (2016). Interfering and facilitating factors in occupational safety: A systematic review of the safety literature between 1980 and 2015. Paper presented at the annual meeting of the Netherlands-Flanders Communication Association, Amsterdam, the Netherlands.

Cornelissen, P. A., \& Van Hoof, J. J. (2017). The Role and Effects of Communication on Occupational Safety: A Review. Paper presented at the International Conference on Occupational Health and Safety, Miami, FL.

\section{Popular publications}

Cornelissen, P. A. (2015). Hebben we ongelukken nodig om gemotiveerd te worden veilig te werken? Veiligheidsnieuws, 47(3), 7-9.

Cornelissen, P. A. (2016). Don't shoot the messenger: Hoe benaderen we meldingsgedrag ten aanzien van veiligheid? Veiligheidsnieuws, 48(2), 1113. 


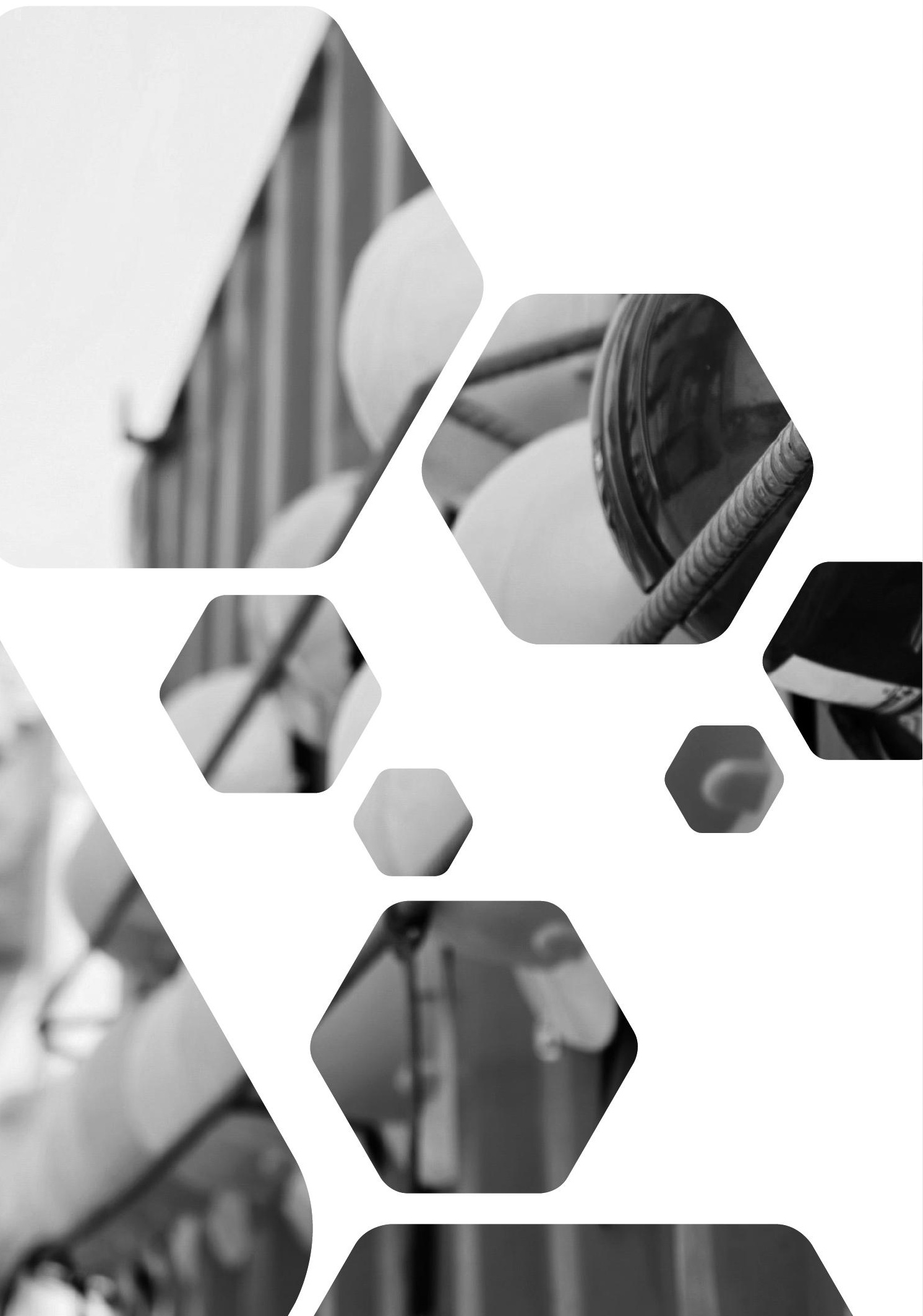




\section{Dankwoord (acknowledgements in Dutch)}

\footnotetext{
'At times our own light goes out and is rekindled by a spark from another person. Each of us has cause to think with deep gratitude of those who have lighted the flame within us.'
}

Albert Schweitzer 


\section{Dankwoord (acknowledgements in Dutch)}

'At times our own light goes out and is rekindled by a spark from another person. Each of us has cause to think with deep gratitude of those who have lighted the flame within us.' - Albert Schweitzer

In de ochtend van 4 augustus 2014 stapte ik voor het eerst sinds lange tijd weer de Cubicus binnen. Waar ik dit de afgelopen jaren duizenden keren had gedaan, was het dit keer anders. Nog steeds student, maar naast student nu ook medewerker. In de vier jaar die volgden heb ik veel steun gehad aan collega's, vrienden en familie en ik wil dan ook van de gelegenheid gebruik maken om iedereen te bedanken die mij op welke wijze dan ook in deze periode heeft gesteund.

In de eerste plaats ben ik Menno de Jong, mijn promotor, erg dankbaar voor zijn tijd, adviezen en vertrouwen. De afgelopen jaren heb je er voor gewaakt dat dit proefschrift niet teveel tot een handboek voor de praktijk is verworden, waar je er tegelijkertijd voor waakte dat we de -in dit proefschrift zo belangrijke- menselijke kant van het verhaal nooit uit het oog verloren. Keer op keer wist je het geheel naar een hoger niveau te brengen door die zaken aan te stippen die voor verbetering vatbaar waren. Daarnaast ben ik Joris van Hoof, mijn co-promoter, dankbaar voor de begeleiding in de aanloop naar dit traject toe en de dagelijkse begeleiding gedurende de afgelopen jaren. Met je pragmatische aanpak wist je de juiste tegenbalans te creëren voor mijn steeds verder uitdijende interesse in gerelateerde onderzoeksgebieden. Ik kon altijd bij je terecht met vragen, opmerkingen en problemen. Bedankt hiervoor! Ook wil ik het Institute for Innovation and Gover-nance Studies, en Irna van der Molen en Kees Aarts in het bijzonder, bedanken voor het mede mogelijk maken van dit proefschrift.

Daarnaast wil ik graag al mijn oud-collega's bedanken. Mijn kamergenoten, Suzanne, Niels, Qian, Silvie en Shu, bedankt voor alle afleidingen, energie, opbeurende woorden, interesse en hulp de afgelopen jaren. Mark, je was er 
bij vanaf het allereerste begin en zag samen met Joris mogelijkheden waar ik ze nog niet had gezien. Dank voor je bijdrages aan artikelen, je aanstekelijke enthousiasme, en voor de heerlijk abstracte discussies. Sikke, bedankt voor alle hulp, de gezellige etentjes en het delen van je overschot aan enthousiasme en energie op de momenten dat ik daar even een gebrek aan had. Susanne en Anique, bedankt voor de koffiemomenten en het relativeren van alle $\mathrm{PhD}$ problemen en natuurlijk jullie kritische en nauwkeurige feedback. Teuntje, bedankt voor het veraangenamen van de vele kilometers die we in de trein moesten maken, de doorlopende competitie en de thee-momenten. Daarnaast wil ik ook al mijn andere (oud-) collega's bedanken voor de goede onderlinge samenwerking, lunchwandelingen, hulp, etentjes en andere gezellige momenten.

De onderzoeken die ik heb uitgevoerd waren niet mogelijk geweest zonder de hulp en medewerking van verschillende organisaties. Fred, Peter, Marc, Jan Kees, Harriët, Willem, José, Bart en Gert, ik wil jullie van harte bedanken voor het mogelijk maken van de verschillende studies.

Ook wil ik al mijn vrienden bedanken voor hun interesse en luisterend oor op momenten dat ik dat nodig had. Bert, ik denk niet dat het zonder jou ooit van promoveren was gekomen. De door jou geregelde afstudeeropdracht bleek de springplank naar dit proefschrift. Bedankt voor je vertrouwen, je oprechte interesse en het luisterend oor dat je ook vanuit Singapore en Wiesbaden bleef bieden. Tim en Tijs, jullie stonden niet alleen aan de vooravond van dit avontuur klaar om mijn vragen te beantwoorden, ook tijdens mijn PhD waren jullie altijd bereikbaar om advies te geven en net wat verder te pushen. Bedankt!

José en Berto, ondanks dat Berto er niet meer is wil ik jullie beiden heel erg bedanken voor jullie steun, interesse in mij en mijn proefschrift. Stephan, ik wil je graag bedanken voor het relativeren van zaken waar ik huize hoog tegen op zag, de gezelligheid gedurende de weekenden in Oldenzaal en op vakanties.

Lieve pap, lieve mam. Ik kan me het als de dag van gisteren herinneren hoe jullie mij naar de UT brachten voor de introductieperiode. Trots maar waarschijnlijk ook een beetje huiverig om mij daar zo achter te laten in het verre Oosten. Ik wil jullie bedanken voor jullie steun, het vertrouwen en de rust die jullie mij tot de dag van vandaag geven. 
Lieve, lieve Roos. Wat moeten de afgelopen jaren soms lastig zijn geweest voor jou. Wekkers om 05:15 en bij tijden zelfs om 04:30, aanhoren wat er allemaal wel en niet goed ging, dealen met de stress over de laatste maanden, uitstellen van vakanties, veranderende plannen en ga zo nog maar even door. Ik ben zo blij dat je dit samen met mij hebt willen doen, dat je er keer op keer voor mij was, de rust die je creëerde en bovenal voor alle mooie momenten die we samen hebben mogen beleven. Ik hoop dat we samen nog veel avonturen gaan beleven! 



\section{SPEAKING OF SAFETY}

THE ROLE OF COMMUNICATION IN MANAGING OCCUPATIONAL SAFETY

\section{PIETER CORNELISSEN}

1. Effective improvement of workplace safety demands a broad and comprehensive approach (this thesis).

2. Employees should learn to recognise and deal with demands that conflict with occupational safety (this thesis).

3. A broader understanding of safety communication is essential for improving occupational safety (this thesis).

4. Communication should not be seen as a quick fix for unsafe workplaces (this thesis).

5. The increasingly important position of the market logic in academia might make academics more efficient, but one should question if efficiency should be a goal in academia in the first place.

6. Replication studies are a necessary evil, protecting both academics and practitioners from departing from false assumptions.

7. If open access is to benefit others than academics, it demands a change in writing style.

8. The scientific journey should be as valued as its destination.

9. Round manhole covers show that convenience, quality and safety can go together.

10. 'We should favour those who willingly and consciously neglect a red light over those who unconsciously pass when the light is green' - Fred Aarsen 


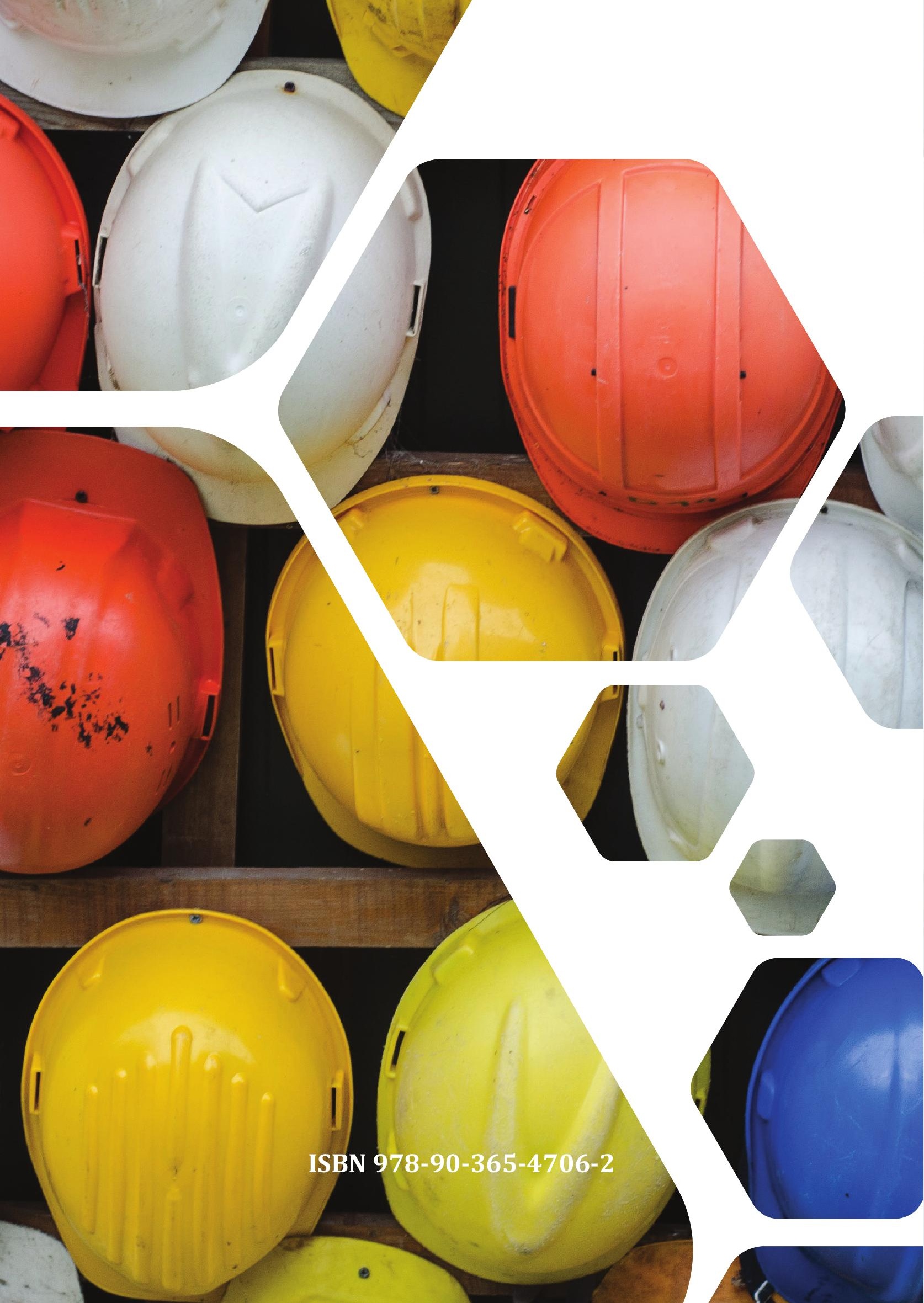

